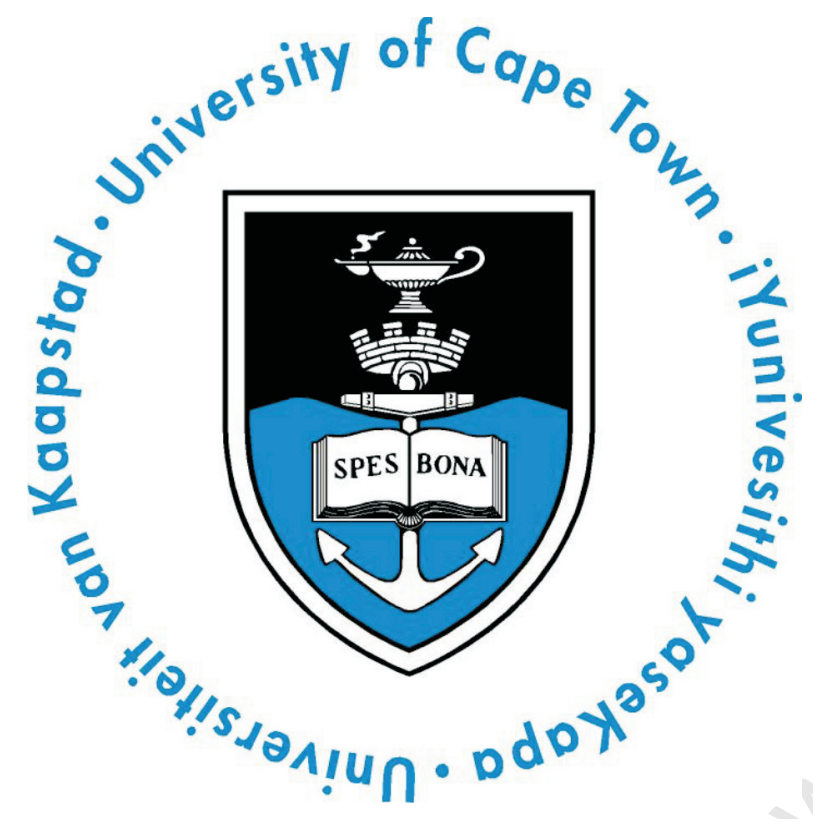

\author{
UNIVERSITY OF CAPE TOWN \\ DEPARTMENT OF HUMAN BIOLOGY \\ DIVISION OF BIOMEDICAL ENGINEERING
}

MASters Dissertation REport

Submitted in the fulfilment of the requirements for the degree of Masters in Biomedical Engineering (by Dissertation)

\title{
An Attempt to Improve Stance Mechanics of Trans-Tibial Amputee Gait by the Design of a Modular Ankle Joint Prosthetic
}

Author:

Alastair B. During DRNALA001
Supervisors:

Associate Professor George Vicatos

Associate Professor Sudesh Sivarasu

September 10, 2018 
The copyright of this thesis vests in the author. No quotation from it or information derived from it is to be published without full acknowledgement of the source. The thesis is to be used for private study or noncommercial research purposes only.

Published by the University of Cape Town (UCT) in terms of the non-exclusive license granted to UCT by the author. 


\section{Plagiarism Declaration:}

- I, Alastair Blaine During, hereby declare that the work on which this dissertation/thesis is based is my original work (except where acknowledgements indicate otherwise) and that neither the whole work nor any part of it has been, is being, or is to be submitted for another degree in this or any other university. I empower the university to reproduce for the purpose of research either the whole or any portion of the contents in any manner whatsoever.

- I have used the American Psychological Association (APA) convention for citation and referencing. Each contribution to, and quotation in, this report from the work(s) of other people has been attributed to, and has been cited and referenced.

- I have not allowed, nor will allow, anyone to copy my work with the intention of passing it off as his or her own work.

\section{Signed: $\quad$ Signed by candidate}

Date: September 10, 2018 


\section{Acknowledgements}

This research was supported by the National Research Fund (NRF). Any opinions, findings, recommendations or conclusions that appear within this dissertation are that of the authors, and therefore the NRF does not accept any accountability in regard thereto.

I would like to give thanks to the following organisations and people, without whom the completion of this dissertation would not have been possible:

To the University of Cape Town, for supporting me and thus allowing me this incredible oppurtunity. To the NRF, for their financial support that helped fund me for these two years.

To my supervisor, Associate Professor George Vicatos, who has been willing to explore new ideas with me since I approached him in 2015 and subsequently granted me the freedom to create the project I am passionate about - for that I will always be grateful. To my co-supervisor Associate Professor Sudesh Sivarasu, for his concise advice and constant support that was stabilising in times of need. I hope to be able to find mentorship in both of you as I embark on my new career.

To Mr. Pierre Smith and his team at UCT's Mechanical Engineering workshop, for the manufacturing of the prototype designs. And to Mr. Charles Harris and his team at the Health Sciences workshop, for their design advice and stellar work in adjusting the prototype into a final working model. To Mr. Eugene Rossouw at the Prosthetic Rehab Clinic, for sharing his insight and advice on the prosthetics field, and for the provision of the Otto Bock 1D10 that was used in this dissertation. To the team at the Western Cape Orthotics and Prosthetics Centre, for sharing their insight into the low-cost prosthetics field and understanding of the challenges experienced by low-income amputees.

To my colleagues in the Division of Biomedical Engineering: Mr. Scott Bruton, alongside whom I undertook this masters challenge, for his enthusiastic assistance and our shared experience; to Mss. Lee Kruse and Mr. Giancarlo Beukes, for reviewing and editing my dissertation; to Mr. Edmund Wessels, Mr. Gokul Nair, Mr. Seth Thompson and Mr. Jonothan Glenday, for their advice and consultation throughout my dissertation; and to the rest of the my colleagues for supporting me over these past two years. To all of my friends and roommates who have supported me through my undergraduate and/or post-graudate years, for your loyalty, friendship and challenging 
honesty, and specifically to Mr. Ryan White, an old friend who so eagerly helped my edit my final draft. And to Mss. Sarah Adam, for the love, friendship and support provided as we shared the post-graduate experience.

And lastly to my parents, Mr. John During and Mrs. Helen During, for emotionally supporting me throughout this time. Without tit, these tough but incredible two years would not have been possible. And to my best friend and brother, Mr. Andrew During, for always believing in me. 


\section{Abstract}

Background: A-priori research shows that trans-tibial (TT) amputees display poor gait parameters when walking with low-cost ankle-foot prosthetics (here referred to as baseline AFP's). This has drastic implications for the amputee populations in the developing world specifically, as they have limited access to advanced prosthetic technologies. Low-cost AFP's are unable to adequately replicate natural stance mechanics, and reliance on these devices results in increased energy expenditure, osteoarthritis and lower-limb joint deterioration.

Methodology: This project details the design of a novel ankle joint prosthetic (AJP) that serves as an attachment to baseline AFP's, with the aim of facilitating better stance mechanics via the restoration of ankle joint mechanisms. The work is presented in three core sections: Part 1 explains the rationale as to why adequately replicating natural stance mechanics is an appropriate need; Part 2 presents the design of the modular low-cost AJP that utilises only simple mechanical elements; and Part 3 presents the experimental quantification of the impact the AJP has on stance mechanics of a baseline AFP (Otto Bock 1D10) in a simulation of the TT amputee walking gait cycle, via the use of three able-bodied participants and a pseudo-prosthesis.

Results: The results indicate that the AJP significantly improves the stance mechanics of the baseline AFP. During forefoot rollover a stable joint moment and an increase in joint range of motion (RoM) was observed, yielding a decrease in ankle stiffness. During initial weight acceptance of early stance, an increase in joint RoM displays the restoration of controlled plantarflexion, which indicates an improved transition from heelstrike to footflat. This is a critical mechanism that facilitates stability control during weight acceptance, and the results suggest that the designed AJP is performing better in this regard than its closest functional competitor. However, equipment errors limited the ability to accurately report on ankle stiffness of this phase.

Conclusions: Overall the final conclusions are that the designed AJP improves rollover shapes of the baseline AFP, eases phase transitions, and facilitates stability control and forward tibial progression. In combination with the low cost price ( \pm 50 USD), its ease of assembly and modular design, the AJP is thus a preferable option for low-income amputees in developing countries. Finally, there is significant evidence of functional and mechanical reliability, and therefore testing of the device can progress to a clinical study involving amputee participants. 


\section{Contents}

\section{Research and Project Design}

1 Background: Lower Limb Amputees in South Africa and the Developing World 3

2 Overview of Natural Stance Mechanics 5

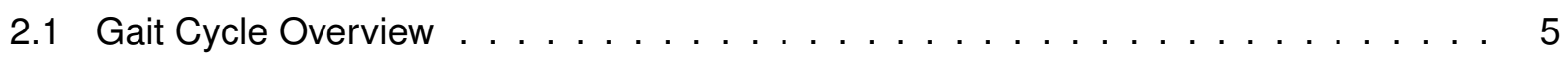

2.2 Weight Acceptance Mechanisms . . . . . . . . . . . . . . . 7

2.3 Role of the Lower Leg Musculature during the Stance Phase $\ldots \ldots$. . . . . 8

3 Trends in Ankle-Foot Prosthetic (AFP) Design 10

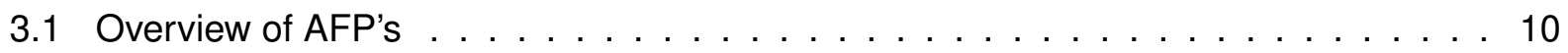

3.2 Attempts to Replicate Weight Acceptance Mechanisms . . . . . . . . . . . . 10

3.3 Attempts to Improve Stance Mechanics using DER's . . . . . . . . . . . . . . 12

3.4 Attempt to Recycle Impact Energy into Push-off Power . . . . . . . . . . . . . . 13

3.5 Attempt to Mimic Musculature Stiffness Control with Flexible Ankle Joint Prosthetic . 14

4 Theoretical Definition of Ankle Joint Mechanisms 19

4.1 Defining the Mechanics of the Ankle in Terms of Simple Mechanical Elements . . . 19

4.2 Core Design Principles . . . . . . . . . . . . . . . . . 20

5 Project Design 2

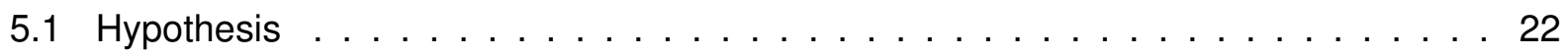

5.2 Study Aims . . . . . . . . . . . . . . . . . . . . . 22

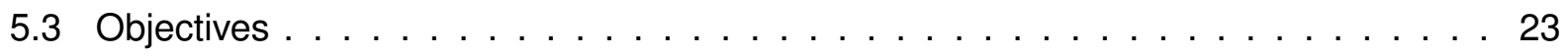

5.4 Scope . . . . . . . . . . . . . . . . . . . 23

5.5 Research and Design Limitations . . . . . . . . . . . . . . . 23

\section{The Design of the Ankle-Joint Prosthetic 25}

6 Design Requirements 26

6.1 General and Functional Requirements of a Passive Mechanical Ankle Prosthetic . . 26

6.1 .1 Core Functional Design Objectives . . . . . . . . . . . . . . . 26

6.1 .2 Ergonomic Related Considerations . . . . . . . . . . . . . . 28

6.2 Quantifying the Kinetic and Kinematic Requirements of an AJP . . . . . . . . . 29 
7 Design Process

7.1 Concept Development . . . . . . . . . . . . . . . . . . . . . . . 32

7.1.1 Concept 1: AJP using Extension Springs . . . . . . . . . . . . . . . . 32

7.1.2 Concept 2: AJP using Compression Springs . . . . . . . . . . . . . . . 34

7.2 Final Design . . . . . . . . . . . . . . . . . . . 36

7.2.1 AJP Housing: Optimising Spring Arrangement . . . . . . . . . . . . . . 36

7.2.2 AJP Springs: Specification and Selection . . . . . . . . . . . . . . . . . . 39

7.2.3 AJP-AFP Interface Methodology . . . . . . . . . . . . . . . . . . . 43

8 Evaluation of Final Design $\quad 45$

8.1 Spring Lifetime . . . . . . . . . . . . . . . . . . . . . . . 46

8.2 Cost Breakdown and Assembly Analysis . . . . . . . . . . . . . . . . . . . 46

8.3 Finite Element Analysis of AJP Housing . . . . . . . . . . . . . . . . . . 47

8.4 Axle Calculations . . . . . . . . . . . . . . . . . . . . 50

\section{Experimental Analysis 52}

9 Experimental Methodology

9.1 Evaluating Prosthetic Devices by the Simulation of Amputee Gait . . . . . . . . . . 56

9.1.1 The Methodology in Literature . . . . . . . . . . . . . . . . . . 56

9.1 .2 The Design of Pseudo-Prosthesis . . . . . . . . . . . . . . . 57

9.2 Study Design . . . . . . . . . . . . . . . . . . . . . . . . 58

9.2.1 Intended Participant Population . . . . . . . . . . . . . . . . . . 58

9.2 .2 Research Apparatus . . . . . . . . . . . . . . . . . . . . . . . . . 58

9.2 .3 Testing Plan . . . . . . . . . . . . . . . . . . . . . . . . . . . . . . . . . . . . .

9.2 .4 Testing Procedure . . . . . . . . . . . . . . . . . . . . . . 61

9.3 Data Analysis Plan . . . . . . . . . . . . . . . . . . . . . . . . . . . . . . . . . . . . . . . . . . . .

9.3 .1 Data Processing . . . . . . . . . . . . . . . . . . 65

9.3 .2 Statistical Analysis . . . . . . . . . . . . . . . . . . 67

10 Experimental Results $\quad 69$

10.1 Intra-Participant Results and Analysis . . . . . . . . . . . . . . . . . . 69

10.1.1 Participant $1 \ldots \ldots \ldots \ldots \ldots \ldots \ldots$. . . . . . . . . . . . . . . . . . . . . . . . . .

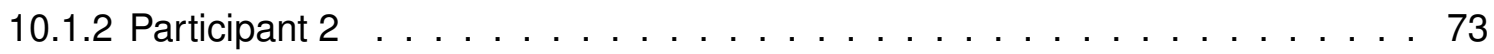

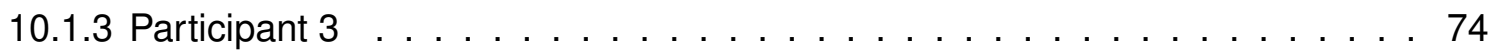

11 Discussion $\quad 76$

11.1 Summary of Results . . . . . . . . . . . . . . . . . . 76

11.2 Comparing the Results to Theoretical Design Aims and Literature . . . . . . . . . 85

11.3 Review of Methodology . . . . . . . . . . . . . . . . . 87

12 Conclusions $\quad 92$ 
$\begin{array}{lr}\text { Appendices } & 99\end{array}$

$\begin{array}{ll}\text { A AJP Theoretical Stiffness Calculations } & \text { A1 }\end{array}$

$\begin{array}{ll}\text { B Axle Strength Calculations } & \text { A4 }\end{array}$

$\begin{array}{ll}\text { C Spring Catalogue Sample (ISO 10243) } & \text { A7 }\end{array}$

$\begin{array}{ll}\text { D AJP Drawings } & \text { A14 }\end{array}$

$\begin{array}{ll}\text { E DPP Drawings } & \text { A22 }\end{array}$

$\begin{array}{ll}\text { F Ethics Approval Letter } & \text { A27 }\end{array}$

$\begin{array}{ll}\text { G Participant Consent Form } & \text { A30 }\end{array}$

H Participant Questionnaire $\quad$ A36

I Statistical Analysis Methodology (Example) $\quad$ A39

$\begin{array}{ll}\text { J Additional Results Figures } & \text { A42 }\end{array}$ 


\section{List of Figures}

1 Dissertation Overview $\ldots \ldots \ldots \ldots \ldots \ldots \ldots \ldots$

2.1 Gait Cycle Overview ([Wagner et al., 1987]) . . . . . . . . . . . . . . 5

2.2 Ankle Mechanics during Able-Bodied Gait [Gates et al., 2004] . . . . . . . . . . . 6

2.3 The Inverted Pendulum Model. [Collins and Kuo, 2010] . . . . . . . . . . . 7

3.1 Delayed Transition to Foot-Flat in Trans-tibial Amputee Gait.[Wagner et al., 1987] . 11

3.2 Single Axis Foot. [WillowWood, 2017] . . . . . . . . . . . . . . . . . 12

3.3 Flex-Foot Assure. [Ossur, 2018]) . . . . . . . . . . . . . . . . . . 13

3.4 The Controlled Energy Storage and Return (CESR).[Collins and Kuo, 2010] . . . . 14

3.5 Endolite Multiflex Ankle. [Endolite, 2017] . . . . . . . . . . . . . . . . . . 15

3.6 Impact of the Multiflex Ankle on Stance Mechanics. [Su et al., 2010] . . . . . . . 17

3.7 Impact of the Multiflex Ankle on Power. [Su et al., 2010] . . . . . . . . . . . 18

4.1 Ankle Angular Position vs moment Characterisation. [Palmer, 1999] . . . . . . 20

7.1 Design Process Flowchart. . . . . . . . . . . . . . . . . . . . 31

7.2 Concept 1: Using Extension Springs. . . . . . . . . . . . . . 33

7.3 Concept 1: Function during CDF. . . . . . . . . . . . . . . . . 34

7.4 Concept 2: Using Compression Springs. . . . . . . . . . . . . . . . . 35

7.5 Design Solution to Improve Balance Control . . . . . . . . . . . . . . . . 37

7.6 Design Solution to Accommodate for Compression Springs in Angular Rotation. . . 39

7.7 Flowchart of the Iterative Spring Specification and Selection Procedure. . . . . . . 40

7.8 Internal AJP Housing Arrangement. . . . . . . . . . . . . . 41

7.9 Recommended Die Spring Assembly. . . . . . . . . . . . . . . 43

7.10 Render of Final AJP Model. . . . . . . . . . . . . . . . . . . . 44

8.1 Manufactured AJP Mounted on SACH Foot. . . . . . . . . . . . . . . . 45

8.2 Results of FEA Stress Simulation under Peak Dorsiflexion Loading Conditions. . . . 48

8.3 Results of FEA Simulation. . . . . . . . . . . . . . . . . . . . 49

8.4 Free Body Diagram (FBD) of Forces and Moments acting on AJP. . . . . . . . . 50

8.5 Free Body Diagram (FBD) of Forces acting AJP Axle. . . . . . . . . . . 50

8.6 Results of Axle Stress and Deflection Calculations. . . . . . . . . . . . . . 51

9.1 The Pseudo-Prosthesis. . . . . . . . . . . . . . . . . . . . . . . . . . 54

9.2 Flowchart of the Experimental Methodology. . . . . . . . . . . . . 55 
9.3 CESR Experimental Procedure. [Collins and Kuo, 2010] . . . . . . . . . . . . 56

9.4 The Prosthetic Foot Loading Apparatus. [Hansen et al., 2000] . . . . . . . . . . . 57

9.5 Kinetic and Kinematic Measurement Apparatus . . . . . . . . . . . . . . . . . 59

9.6 The Simulation of Trans-Tibial Amputee Gait . . . . . . . . . . . . . . . . 60

9.7 The Deactivated Pseudo-Prosthesis (DPP) and the Pseudo-Prosthesis (DPP). . . 61

9.8 Flowchart of the Testing Procedure and Data Analysis Plan. . . . . . . . . . . . . 62

9.9 The Motion Capture Marker System. . . . . . . . . . . . . . . . . . . . . . 64

9.10 Visual Representation of Ankle Moment Calculation. . . . . . . . . . . . . . . . 66

10.1 Period of Stance vs Period of Stance of Participant 2. . . . . . . . . . . . 70

10.2 Period of Stance vs Ankle Moment of Participant 2 . . . . . . . . . . . . . . . 71

11.1 Kinetic and Kinematic Changes and Trends. . . . . . . . . . . . . . . . . 77

11.2 Temporal-Spatial Changes and Trends. . . . . . . . . . . . . . . . 78

11.3 Foot Angle during Stance. . . . . . . . . . . . . . . . . . . . . . . 79

11.4 Ankle Angle vs Ankle Moment of Participant 2. . . . . . . . . . . . . . . 82

11.5 Vertical Ground Reaction Force over Period of Stance of Participant 2. . . . . . . . 83

11.6 Fore-Aft Ground Reaction Force over Period of Stance of Participant 2. . . . . . . 84

11.7 Location of CoP Relative to Ankle Position in Sagittal Plane. . . . . . . . . . . . . 88

\section{List of Tables}

3.1 The Effect of the Multiflex Ankle on Stance Mechanics. Data from Su et al. [2010]. . 16

6.1 Able Bodied Stance Mechanics (sourced from Su et al. [2010]; Gard [2011]). . . . . 29

7.1 Selected Die Spring Specifications. . . . . . . . . . . . . . . . . . . . . 42

7.2 Theoretical AJP Stiffness. . . . . . . . . . . . . . . . . . . . . 43

8.1 Cost Breakdown of AJP Prototype. . . . . . . . . . . . . . . . . 46

8.2 Peak Load Conditions . . . . . . . . . . . . . . . . . . . . . . . . 47

10.1 Participant Demographics. . . . . . . . . . . . . . . . . . . . . . . 69

10.2 Temporal-Spatial Results and Analysis of Participant 1. . . . . . . . . . 72

10.3 Kinetic and Kinematic Results and Analysis of Participant 1 . . . . . . . . . . . 72

10.4 Temporal-Spatial Results and Analysis of Participant 2. . . . . . . . . . 73

10.5 Kinetic and Kinematic Results and Analysis of Participant 2 . . . . . . . . . . . . 74

10.6 Temporal-Spatial Results and Analysis of Participant 3. . . . . . . . . . 75

10.7 Kinetic and Kinematic Results and Analysis of Participant 3. . . . . . . . . . . 75 


\title{
List of Acronyms
}

\author{
AB $\ldots \ldots \ldots \ldots$ Able-bodied \\ AFP .......... Ankle-Foot Prosthetic \\ AIB .......... Ankle Immobiliser Boot \\ AJP $\ldots \ldots \ldots \ldots$ Ankle Joint Prosthetic \\ BW ........... Body weight \\ CDF .......... Controlled Dorsiflexion \\ CESR ........ Controlled Energy Storage and Return \\ CoM ......... Centre of mass \\ CON ........... Control Test \\ CoP $\ldots \ldots \ldots \ldots$ Centre of pressure \\ CoR .......... Centre of rotation \\ CPF ........... Controlled Plantarflexion \\ DPP .......... Deactivated pseudo-prosthesis \\ DoH ......... Department of Health \\ EXP $\ldots \ldots \ldots \ldots$ Experimental Test \\ FBD $\ldots \ldots \ldots \ldots$ Free body diagram \\ FDO $\ldots \ldots \ldots \ldots$ Functional design objectives \\ FEA $\ldots \ldots \ldots \ldots$ Finite element analysis \\ FP $\ldots \ldots \ldots \ldots$ Force plate \\ HREC ......... Human Research and Ethics Committee \\ IDF ........... International Diabetes Foundation
}


ISO International Organization for Standardization

KK ........... Kinetic and kinematic

LLA ........... Lower limb amputation

N/A ......... Not applicable

PA ............ Prosthetic attachments

PFLA .......... Prosthetic Foot Loading Apparatus

PP ............ Pseudo-prosthesis

PPF .......... Powered Plantarflexion

PVD ........... Peripheral vascular disease

RoM .......... Range of motion

SA $\ldots \ldots \ldots \ldots$ South Africa

SACH ......... Solid Ankle Cushioned Heel

S-W ........... Shapiro-Wilk

TS ............ Temporal-spatial

TT ............ Trans-tibial

UCT .......... University of Cape Town

WHO .......... World Health Organization 


\section{Dissertation Overview}

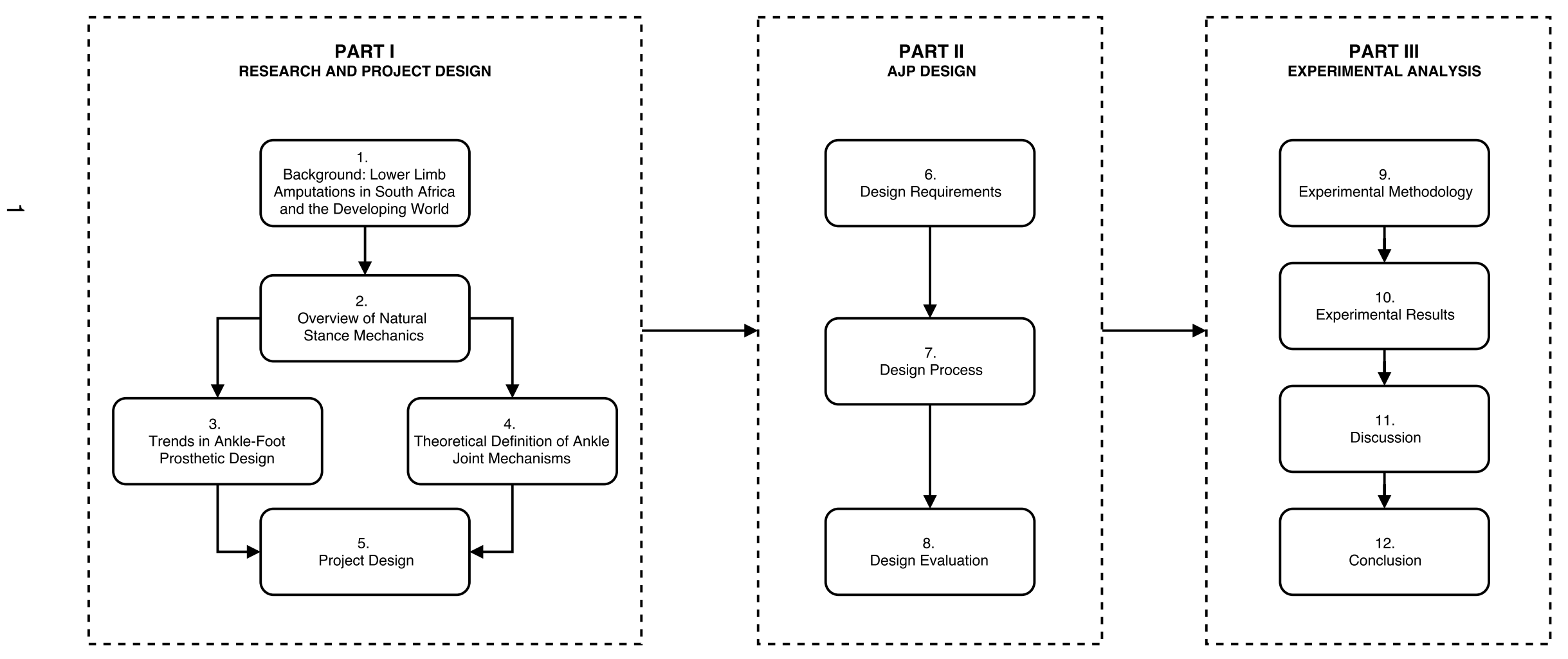

Figure 1: Dissertation Overview 


\section{Part I}

Research and Project Design 


\section{Chapter 1}

\section{Background: Lower Limb Amputees in South Africa and the Developing World}

The collection of data relating to the epidemiology of lower limb amputations (LLA) in the South African (SA) health sector is an almost non-existent practice [Godlwana et al., 2008], and thus clear definitions thereof are near impossible to statistically quantify. Instead, vague definitions of this epidemiology stem from evidence provided by localised cases, and overview critiques of the state of healthcare in the developing world by international bodies such as the World Health Organisation (WHO) and the International Diabetes Foundation (IDF).

Isolated studies on the epidemiology and etiology of LLA's conducted in SA reveal that dysvascular complications account for the majority of cases [Henry, 1993] ${ }^{1}$ [Ellis, 2016]. Studies also show that most traumatic amputations in SA occur at an early age whilst diabetic related amputations occur later in life [Kubheka, 1993]. This is consistent with global trends. An American study documented that dysvascular complications accounted for $82 \%$ of limb amputations in a time period between 1988 and 1996, at a rate that increased by $27 \%$ in that same period [Dillingham et al., 1996]. In a later study Dillingham et al. [2002] quantified the epidemiology of peripheral vascular disease (PVD) related limb amputations in the USA to be at an average annual rate of 44.3 per 100000 population. Yet the relationship between diabetes and LLA is significantly worse in developing countries, where $2 \%$ of diabetics develop PVD and $1 \%$ of people with diabetes undergo a LLA at some point in their lifetime [Boulton et al., 2004]. According to the International Diabetes Foundation (IDF), one in six diabetics in developing countries will develope a foot ulcer during their lifetime, and that $85 \%$ of all diabetes related amputations are preceded by foot ulcers Bakker et al. [2005]. Lack of sanitation and barefoot walking over rough terrain are contributing causes to the development of the diabetic foot in these regions [Boulton et al., 2004]. The IDF also estimates that globally one lower limb is lost to diabetes every thirty seconds [Bakker et al., 2005], the majority of which occur in Africa which has diabetic population of more than 14 million people, although indications are that $78 \%$ of African diabetics are undiagnosed [International Diabetes Federation,

\footnotetext{
${ }^{1}$ An unpublished thesis that was sourced by the National Research Foundation and reviewed by [Godlwana et al., 2008]
} 
2011a]. Locally, the estimated SA diabetic prevalence of $7 \%$ is one of the worst in the continent [International Diabetes Federation, 2011b]. Globally the incidence of diabetes is expected to double by 2030 [International Diabetes Federation, 2011a], but this increase is predicted to be greater in developing regions due to the higher rate of population growth Wild et al. [2004].

It is therefore clearly evident that the SA Department of Health $(\mathrm{DoH})$ is going to experience an exponential increase in diabetes associated complications over the next few decades, which presents the a significant primary healthcare financial burden. Further, the growth of an LLA population in SA presents a major secondary financial burden on the $\mathrm{DoH}$, due to the negative impact on the psychological condition and the economic implications thereof. This has also been found to ring true with the local population Kubheka [1993]. A major contributing cause to negative psychological and economical associations with LLA is the quality of the lower limb prosthetics distributed in developing countries. Amputees in developing countries typically require use of their prosthetics more than their counterparts in developed countries, which hastens the rate of deterioration. Furthermore, the poor quality of these devices and their poor suitability to rural environments often results in devices being used in a state of disrepair and/or not being used at all [Magnusson et al., $2014,2013]$, although this may in part also be due to lack of access to servicing. Thus, while preventative care is an absolute priority, rehabilitative care is of equal importance.

When considering that almost all prosthetics are imported from developed countries, it is not surprising that the $\mathrm{DoH}$ is limited to providing only the lowest cost prosthetics, whilst those of lowto mid-income can barely afford to purchase any devices of a higher grade. The most basic of prosthetic devices (SACH foot), which is the only prosthetic foot distributed by the Western Cape Prosthetics and Orthotics Centre, is purchased by the centre at a cost price of approximately \pm $R 2,000$ ( $\$ 150)$, whilst a bottom bracket private sector device will cost a client $\pm R 25,000(\$ 2,000)$. This, therefore, leads to the conclusion that there is a pressing need to ease the initial and long term financial burden on the DoH by providing cost effective prosthetic solutions, whilst simultaneously improving the quality of life of the amputees that rely on these supply chains. It is thus theorised that both of these goals can be achieved by: (i) improving the suitability of prosthetics to developing environments; (ii) improving the durability of the devices (and thus lengthen their lifespan); (iii) producing devices that meet the physical needs of this population and by (iv) considering socio-economic factors that might be important to these population groups. It is likewise important to consider factors relating to the characteristics of the public health sector in SA. Producing prosthetic devices in SA will only aid the attempt to raise the quality of living of local amputees if the device is able to seamlessly integrate with the current supply chain and provision methodologies used by the DoH. 


\section{Chapter 2}

\section{Overview of Natural Stance Mechanics}

\subsection{Gait Cycle Overview}

This section describes an overview of the normal walking gait cycle. It focuses on the kinematics of in the sagittal plane. Additionally, this section yields a broad relative overview of concepts and terminology.

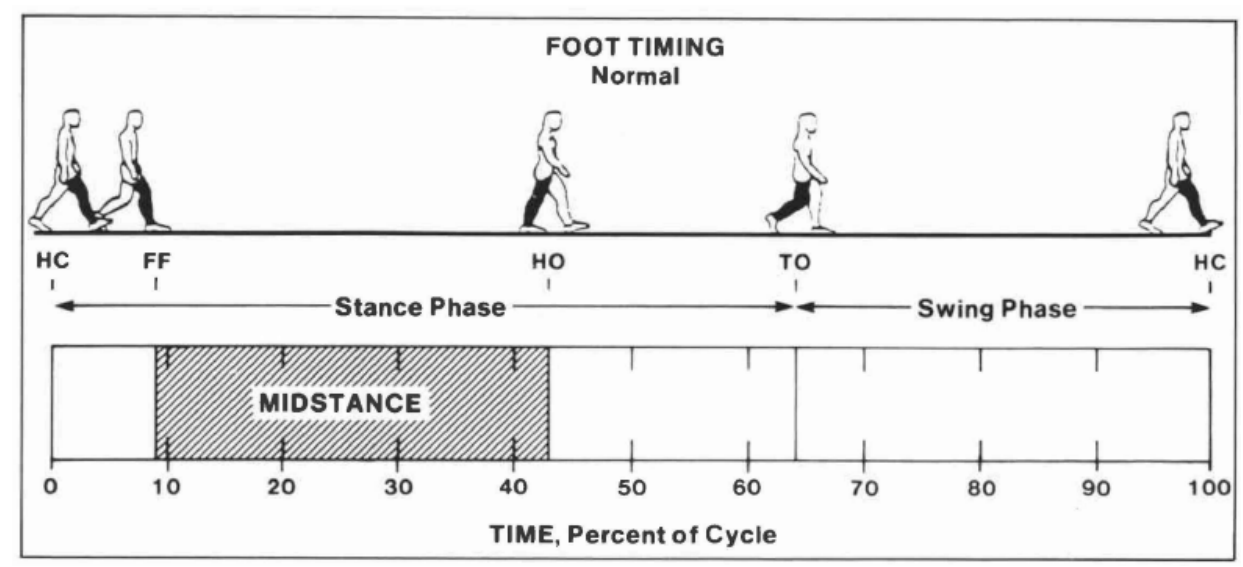

Figure 2.1: Gait Cycle Overview ([Wagner et al., 1987])

This image illustrates a typical representation of the gait cycle, and is phase dependant rather than time dependant. The cycle begins with heel contact of one heel, and terminates when that same heel makes in subsequent heel strike. The stance phase (0-65 percent) runs from heelstrike to toe off and includes the transition to footflat. The swing phase (65 - 100 percent) runs from toe-off to secondary heelstrike

The gait cycle is a term used to define one full stride, standardised as the transition from initial heel contact $(0 \%)$ to the successive heel contact of the same foot (100\%). As shown in Figure 2.1, the gait cycle is initiated at heel contact, shortly followed by footflat. This transition falls within the early stance phase. Footflat represents the start of mid-stance phase, which runs from footflat to heel rise. Push-off work occurs during late-stance between heel rise and toe-off. Initiation of heel rise roughly corresponds to the heel contact of the contra-lateral limb. Thus, double limb support 
occurs roughly between initiation of heel rise and toe-off of the stance limb. [Wagner et al., 1987]

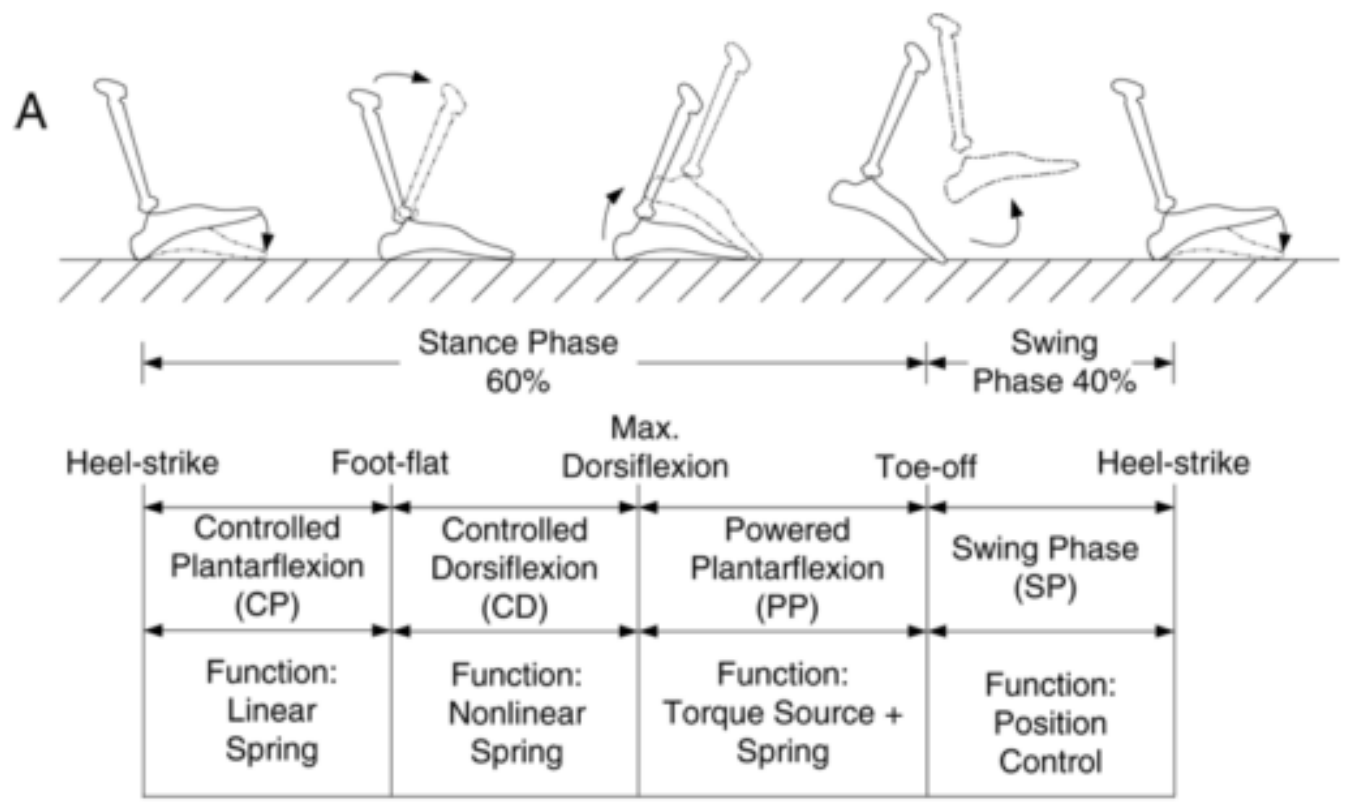

Figure 2.2: Ankle Mechanics during Able-Bodied Gait [Gates et al., 2004]

This figure provides visual aides for the description of the transition phases evident during the stance phase of the gait cycle. Observe the relative angular rotation between the tibia and the foot during level-ground walking of able-bodied $(A B)$ gait. Of specific interest is the stance phase, catergorised into three sub-phases: CPF; CDF; PPF. The mechanical characterisation of these phases is discussed in Section 4.1.

Gates et al. [2004] demonstrates that the ankle joint mechanics during the stance phase can be based on a principle of two rigid bodies rotating relative to each other (the tibia rotating relative to the calcaneal-metatarsal line in the foot), and thus that the stance phase can be described by three linear subphases: Controlled Plantarflexion (CPF), Controlled Dorsiflexion (CDF) and Powered Plantarflexion (PPF). Figure 2.2 illustrates these three sub-phases, all of which can be modelled as various stages of spring displacement [Gates et al., 2004] [Palmer, 1999], and are the core focus of this literature review. They can be described as follows:

1) CPF initiates at heelstrike, and controls the ankle through plantar flexion until footflat is reached.

2) CDF initiates at the termination of CPF, and is characterised by the control tibial forward progression as the lower leg rolls over the planted ankle.

3) PPF initiates at the termination of tibial progression, as the heel leaves the ground. In natural mechanics this is an active process that loads the forefoot, proceeding to the powered push-off that operates via powered plantar flexion. 


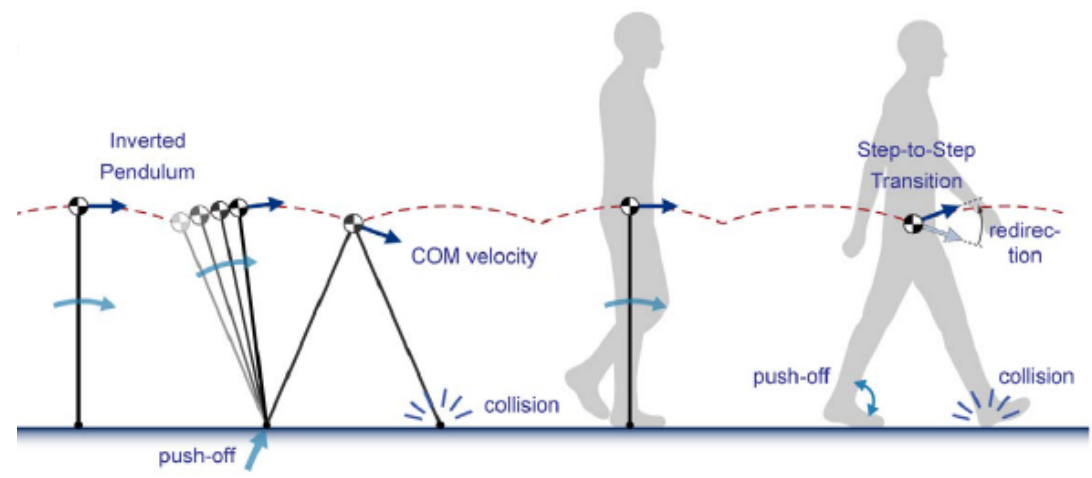

Figure 2.3: The Inverted Pendulum Model. [Collins and Kuo, 2010]

An alternative academic model of the gait cycle describes the kinematics of the legs during walking is that of the inverted pendulum (Figure 2.3), which simplifies walking by making a stiff-leg assumption, and provides excellent insight into the potential-to-kinetic energy conversion principles walking [Zajac et al., 2003] [Collins and Kuo, 2010]. However, whilst this model is an appropriate high-level estimation for the gait of a AB subjects, it is limited by the fact that it does not take into account the co-ordination and action principles between leg muscle groups [Zajac et al., 2003]. Thus this model fails when attempting to breakdown the gait cycle of subject with impaired or nonexistent lower leg, as it cannot explain the relevance of the missing ankle joint co-ordination that results from the amputation.

\subsection{Weight Acceptance Mechanisms}

During walking locomotion, the first point of ground contact for the focus leg is at the heel. Consequently, the impact energy imparted on the heel is rapid and abrupt, and must be absorbed by the body. In an intact limb, this is achieved via CPF, which performs by thee dynamics of relative controlled joint movement and activated muscle groups [Perry et al., 1997; Aerts et al., 1995; Neptune et al., 2001; Kepple et al., 1997]. Further, the mechanical process of accepting body weight impact is equally important in stability control. The weight transfer from the rear contra-lateral limb onto the forward stance leg is a finely tuned procedure that relies on on co-ordinated joint movement [Zajac et al., 2003; Neptune et al., 2001; Waters and Mulroy, 1999; Kepple et al., 1997].

Key to stability is the length of time that the subjects body weight is solely supported by the heel, a relationship that is inversely proportional. In the normal gait of an intact limb, heelstrike occurs with the ankle joint in an approximately neutral position relative to the tibia. Joint mobility during CPF then allows the ankle to plantarflex 12 to $15^{\circ}$ so as to reach footflat relatively fast, rapidly increasing the base area of support to allow for the transfer of body weight onto the stance limb [Perry et al., 1997; Breakey, 1976]. This is the kinetics of the CPF process described in Section 
2.1 , and is the first major shock absorption mechanism of early stance.

\subsection{Role of the Lower Leg Musculature during the Stance Phase}

Reviewed literature explains how the dorsiflexor and plantarflexor musculature play core roles in the control the stance mechanisms described in Section 2.1 and 2.2. Brockett and Chapman [2016] described the musculature mechanisms used to control CPF, CDF and PPF: during CPF the dorsiflexor muslces apply eccentric muscle force to control transition to foot flat; during CDF the plantarflexors apply eccentric muscle force to control tibial progression; and during PPF the planterflexors undergo concentric contraction in order to provide power for toe-off. In terms of CPF, Perry et al. [1997] discusses how early slowing of the foot is the first shock absorption mechanism after heel contact, and is predominantly controlled by the anterior tibialis and long toe extensors. This muscle activity both limits impact shock at footflat, but also aids conservation of momentum.

In terms of CDF and PPF control, Neptune et al. [2001] found that the uni-articular soleus and bi-articular gastrocnemius muscles (i.e. the calf muscles) are activated from early stance phase right through to pre-swing in late stance, increasing and decreasing vertical trunk energy before and after mid-stance, respectively. The data generated by Neptune et al. [2001] showed that while the quadriceps femoris muscles is the primary contributor to knee stability during early and late stance transitions, the soleus is the only muscle to provide stability throughout stance. Specifically during CDF, the body weight (BW) shifts onto the forefoot and creates a pivot over which the body can progress during the mid-to-late stance transition, and the soleus controls tibial progression by applying an antagonist deceleration force as the tibia rotates through its neutral position following footflat [Neptune et al., 2001]. The shift of weight simultaneously initiates the forward swing of the trailing leg, onto which body weight will be transferred once secondary ground contact is made. The summed effect of the soleus and gastrocnemius during PPF is to accelerate the foot into plantar flexion, via simultaneous flexion of the knee and vertical raising of the heel. This is required later to adequately perform push-off work incurred during PPF [Zajac et al., 2003]. The collective conclusions indicate that the plantar flexors dominate support of the trunk and limb during the stance phase, providing support for the transfer of power through the musculo-skeletal system.[Kepple et al., 1997; Neptune et al., 2001; Zajac et al., 2003]. Impairment or loss of these muscles would therefore result in an inability to control CDF and PPF, leading to reduced knee flexion and prolonged compensation on the part of the vastus muscle group.

In terms of metabolic impact, Neptune et al. [2001] found that the energy consumption of these plantarflexors during stance progression is expected to be low. A primary reason is that the efficient use of lower limb joint muscles is characterised by minimum change in length of muscle fibres, approaching isometric conditions [Waters and Mulroy, 1999]. Yet, a characteristic of the plantar flexors during stance activity is that of energy storage and return. Neptune et al. [2001] showed that, due to their considerable lengths, both the soleus and the gastrocnemius store considerable amounts of early stance energy in the aponeuroses and calcaneal tendon of the foot. This energy is returned in late stance to aid trunk forward progression and initiate push-off of the 
stance leg during PPF. Kuo [2001] argued that the most productive time to release the stored energy is during late stance, just prior to toe-off. Important to note is that the release of this energy first requires loading of the forefoot, a process that transpires during CDF. However, it is still important to acknowledge that in an intact limb the PPF is a net positive process, and thus cannot be completely mimicked via the recycling of energy.

In summary, the activity of the dorsiflexors and plantar flexors around the ankle during stance actively recycles the flow of energy from early stance to late stance, and dominates work output. It is therefore theorised that the loss of muscle and joint mechanisms in a TT amputee leads to compensatory actions of the upper-leg muscle groups, which is expected to increase the metabolic energy consumption [Waters and Mulroy, 1999]. Combined with the indication that loading of the forefoot is initiated during CDF, this reveals that a lack of adequate functional replacements in prosthetic or orthotic design would lead to gait cycles that deviate from normal and would thus be characterised by:

i) Passive and delayed plantar flexion, relying instead on momentum to initiate transition to footflat, which was further discussed in Section 2.2. This yields reduced stability control.

ii) Excessive stability compensation, using alternative muscles such as the vastus muscle group.

iii) Limited ability to control CDF, leading to reduced energy loading of the forefoot for push-off.

iv) Inability to actively perform PPF, resulting in net balanced (at best) or negative ankle power. 


\section{Chapter 3}

\section{Trends in Ankle-Foot Prosthetic (AFP) Design}

\subsection{Overview of AFP's}

Most AFP's discussed in this study can be broadly categorised into two categories.

'Baseline' AFP's are the most basic on the market, and are typically prescribed to low-income amputees and low-activity users. The AFP's in this category consists predominantly of solid ankle cushioned heel (SACH) feet and, a slight upgrade thereof, SACH-Dynamic feet. SACH feet has no moving parts, and a rigid internal keel, and relies on rubber or foam regions to deform under load in order to perform the minimum required functions. They are durable, stable and inexpensive, and are thus ideal for externally funded prescription to low-income amputees in developing regions. However, they are also inflexible, cannot be customised and offer minimal shock absorption. The $\mathrm{SACH}$-Dynamic feet differ from SACH feet only in that the keel is more flexible and thus perform better under forefoot loading [NZALS, 2018].

Dynamic Energy Response (DER) feet introduce a pseudo-spring system into the anatomical forefoot of the prosthetic (by the introduction of a carbon fibre blade), in an attempt to passively mimic forefoot dorsiflexion. These feet improve forefoot rollover shapes to a better degree than the SACH-Dynamic, and the pseudo-spring nature of the blades allows for the return of some of the energy stored during loading of the forefoot. These feet offer better shock absorption, are lightweight and adapt better to uneven terrain [NZALS, 2018]. This means that DER feet are better suited to amputees in developing regions, were not for the fact that they are priced out of the market.

\subsection{Attempts to Replicate Weight Acceptance Mechanisms}

Perry et al. [1997] analysed the weight acceptance mechanics of TT amputees in both baseline and DER AFP's, with the specific intention of identifying causes of instability in TT amputee gait. The major findings of this study is that the nature of zero or uncontrolled relative joint motion between the heel of the feet and tibia in both categories of feet leads to (i) a delay transition from 
heel contact to footflat, which causes (ii) diminished and delayed knee flexion. To elaborate on the former, the immobilisation of the ankle joint in a AFP design leads to a lack of artificial plantarflexion. Therefore relative angular rotation between the foot and tibia cannot occur, and thus the transition to footflat is delayed until the tibia can progress to an upright position [Perry et al., 1997; Wagner et al., 1987], as illustrated by Figure 3.1. Interestingly the SACH displayed a greater capacity for plantarflexion during early stance than the DER's [Wagner et al., 1987], but only half that of the natural ankle. Consequentially TT amputees adjust gait characteristics to minimise impact loading, torque and bending stress on their residual limb, using mechanisms such as flat foot ground contact [Gates et al., 2012; Wirta et al., 1991; Gailey et al., 2008]. These studies further suggest that these transition delays could be causing increased reliability on the hip and knee muscle groups, which could be a major contributor to the increased energy expenditure of TT amputees. Additionally, these studies have shown that the gait adjustments have negative long term consequences, with Gailey et al. [2008] examining the development of osteoarthritis of the knee and/or hip joints of both the intact and amputated limb.
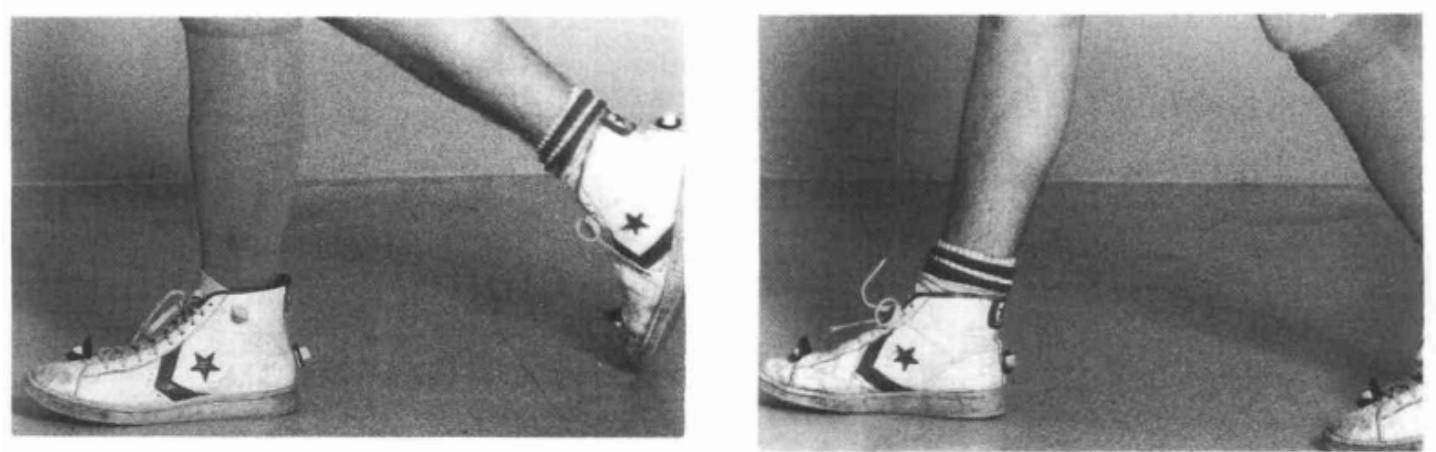

Figure 3.1: Delayed Transition to Foot-Flat in Trans-tibial Amputee Gait.[Wagner et al., 1987]

This figure displays the difference in plantarflexion mobility during early stance between a prosthetic limb (left image) and an intact limb (right image). Notice the position of the tibia when footflat occurs in the prosthetic, versus that of an intact limb.

Perry et al. [1997] found that equally as important as the provision of post-heel strike plantarflexion is the restraint thereof, via the analysis of a Single Axis foot (Medi; Germany) that is displayed in Figure 3.2. The Single-Axis provides ankle mobility via a sagittal plane ankle hinge, whereby the joint motion range of rotation is limited by rubber bumpers, in an attempt to allow for relative motion between the prosthetic tibia and foot. Yet, the analysis thereof shows that this AFP mechanism is characterised by an abrupt and uncontrolled free arc of plantarflexion during the transition to footflat. Further, in the analysis of gait mechanics of all three AFPs Perry et al. [1997] found that the consequence of both uncontrolled and rigid ankle motion results in knee flexion half that of the intact limb. This is reflective of the delay in tibial advancement, leading to instability thereof and excessive reliance on a stiffened knee in order to the lock the leg of the amputee.

Thus, it is concluded that a median between the two operating principles needs to be found via 

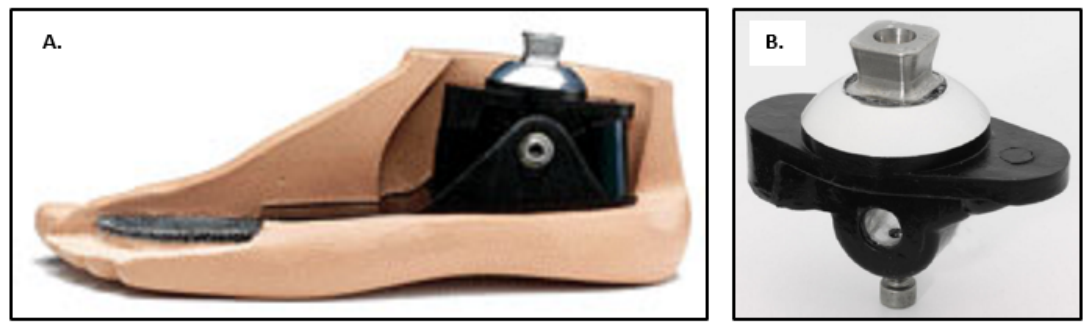

Figure 3.2: Single Axis Foot. [WillowWood, 2017]

The Single Axis foot (A) predominantly provides angular rotation in the sagittal plane, but with limited stiffness control. The ankle (B) operates around a central pivot, whilst the angular rotation range is limited by rubber bumpers [Perry et al., 1997].

the inclusion of mechanisms that will promote controlled rather than uncontrolled plantarflexion transition to footflat, as Perry et al. [1997] concluded such inclusions are needed in order to maintain stability during amputee gait cycles. These inclusions may additionally reduce loading on the residual limb, complement the dynamic joint movement of the hip and knee, and reduce energy expenditure requirements of the amputee.

\subsection{Attempts to Improve Stance Mechanics using DER's}

DER feet attempt to improve stance mechanics and energy efficiency of a trans-tibial amputees by the introduction of a flexible forefoot (often carbon fibre). Functionally, the loading experienced by the forefoot as mid-stance initiates is first elastically absorbed, and then idealistically returned as the amputee engages in toe-off [Wagner et al., 1987]. The loading of the DER keels perform the additional function of allowing more dorsiflextion than baseline AFP's. Wagner et al. [1987] explains that a DER device (Flex-Foot Assur (Ossur; Iceland), seen in Figure 3.3) can perform $20^{\circ}$ of relative ankle joint motion during late stance, in comparison to the $11^{\circ}$ of a SACH foot. This yields increased symmetry in uni-lateral TT amputees, corresponding to improved residual limb loading characteristics. [Schmalz et al., 2002; Su et al., 2010]

The DER designs would hypothetically then result in (i) a greater energy return efficiency of the forefoot keel, although the energy return available is limited to the amount that was passively stored during the CDF transition, and (ii) an efficiency increase in metabolic rates, when compared to a control experiment wherein the subject uses a non-DER prosthetic. And indeed a DER device shows a $57 \%$ energy return rate, compared to the $33 \%$ energy return of a stiff keel baseline AFP, despite indicating no differences in cadence, average velocity or swing period in [Barr et al., 1992]. However, Torburn et al. [1995]'s analysis of the variation in metabolic rates and gait patterns across four DER devices and a baseline AFP finds that the average metabolic rate per metre travelled improves by $4.35 \%$ when using the four DER devices, with the largest individual 


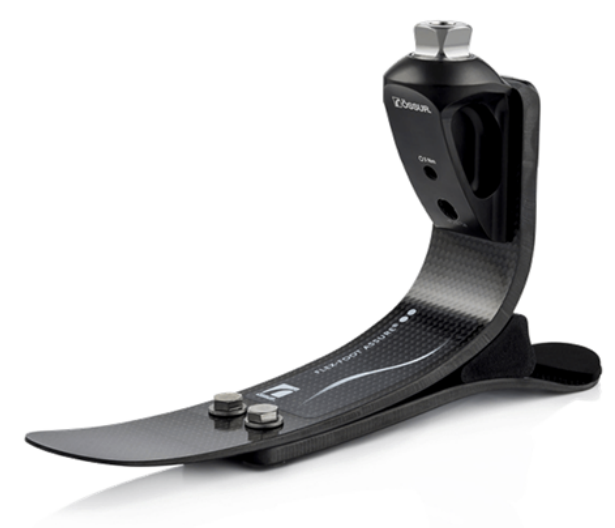

Figure 3.3: Flex-Foot Assure. [Ossur, 2018])

The Flex-Foot Assure provides a cushioned heel and flexible forefoot that intends to improve stance mechanics and gait energy efficiency of amputees during mid-to-late stance.

improvement being $8.8 \%{ }^{1}$ Schmalz et al. [2002]'s analysis of five AFP's of increasing forefoot stiffness and the effect thereof on oxygen consumption of amputees shows that there is no significant correlation between decreased stiffness and reduced energy consumption. It is also shown that the amputees display the same oxygen consumption rates as $A B$ subjects when standing, but a $25 \%$ increase in consumption when walking Schmalz et al. [2002]. Thus, whilst comparative studies of DER's do prove increased dynamic energy efficiency, the minimal increase in metabolic energy efficiency suggests that at walking gait speeds the peak energy costs of the gait cycle are occurring elsewhere.

\subsection{Attempt to Recycle Impact Energy into Push-off Power}

Based on the indication that poor impact absorption at initial impact may play a significant role in energy losses, and the indication that DER devices are not significantly reducing energy consumption, Collins and Kuo [2010] attempted to simultaneously solve both highlighted issues. The authors theorised that by using a spring-and-clutch mechanism, the impact energy incurred at heel strike can be stored and returned during push-off. This would then yield a reduction in energy expenditure of the amputee. Figure 3.4 displays the developed device as well as providing an overview of the devices operation. The internal validation study carried out by the authors used 11 healthy male non-amputee volunteers, and tested them under three conditions: (i) under free ambulation conditions; (ii) using a conventional DER prosthetic (the Seattle Lightfoot II); and (iii) using the CESR device.

For normal intact limb walking, the average value of push-off work is $17.7 \pm 3.4 \mathrm{~W}$. In contrast,

\footnotetext{
${ }^{1}$ Note that this study conducted a parallel experiment using 9 dyvascular amputees, which showed a metabolic rate decrease of 4.3 percent when using the same DER prosthetics.
} 


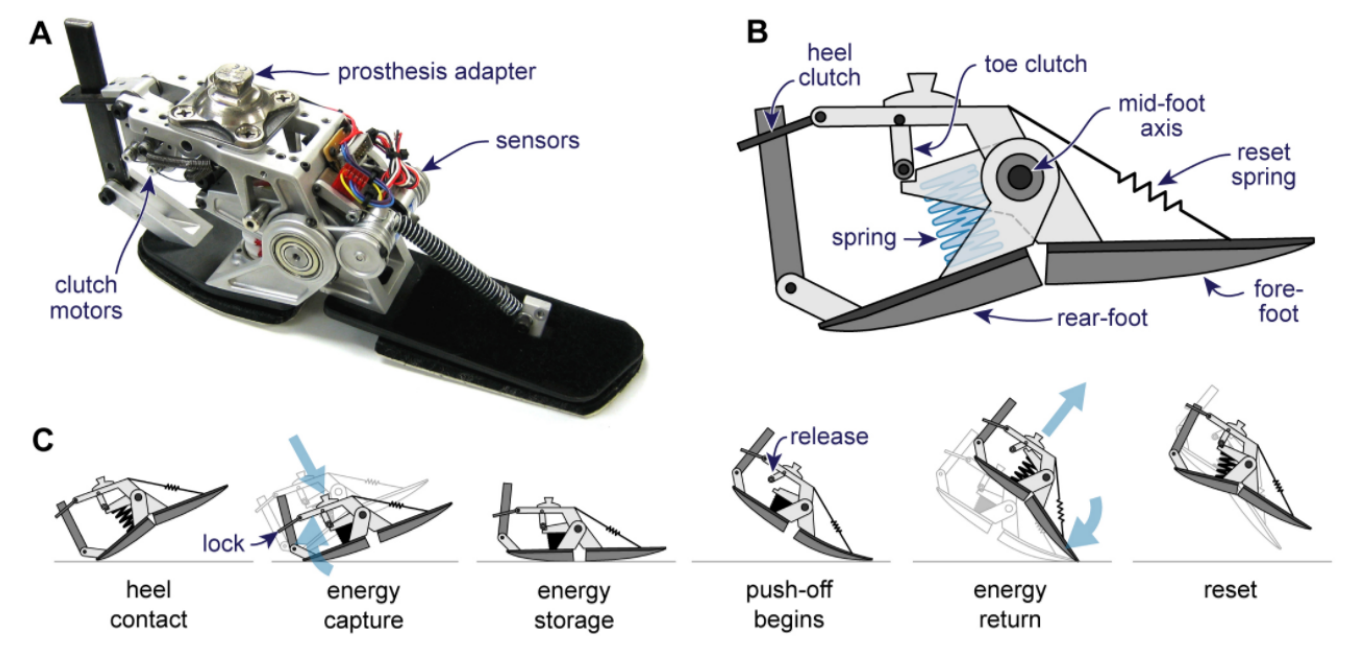

Figure 3.4: The Controlled Energy Storage and Return (CESR).[Collins and Kuo, 2010]

At heelstrike, energy is stored via compression of a spring, which is then locked into place by a clutch. The spring is then released during toe push-off, in an attempt to decrease energy requirements of the amputee. Note that there is no allowance for relative motion between the tibia and the core of the CESR

the CESR generates $18.9 \pm 1.5 \mathrm{~W}$ of push-off work, implying that the CESR actually produces more push-off work than an intact limb. Note that the standard DER prosthetic yields results of $9.8 \pm 1.4 \mathrm{~W}$, almost half that of the intact limb, which suggests that the CESR device should raise the metabolic efficiency of the subject to normal levels. However, amputees using the CESR still show a $14 \%$ increase in net metabolic rate. In comparison, the standard DER prosthetic yielded an average $23 \%$ increase. Therefore, despite raising the push off work to normal levels, the CESR is not proportionally reducing the net metabolic rate. Collins and Kuo [2010] suggest that these continued losses could be due to (amongst others) (i) imprecise capture of energy or (ii) poor timing of spring release during push-off, leading to additional work requirements of the amputated leg.

Due to the relative lack of success in both this and DER designs (see Section 3.3), it is thus theorised that the bulk of energy losses of TT gait cycle is occurring due to a lack of controlled relative ankle joint mobility described in Section 2.1 and 2.2. This theory is supported further by Vanderpool et al. [2008], where a study on the effect of ankle immobilisation on energy expenditure showed that active push-off can be eliminated with little effect on metabolic rate, suggesting that toe-off can be a passive process.

\subsection{Attempt to Mimic Musculature Stiffness Control with Flexible Ankle Joint Prosthetic}

Whilst the Single Axis AFP (discussed in Section 3.2) attempted to mimic the relative angular rotations between tibia and foot, the device fails to mimic musculature stiffness control discussed in 
Section 2.3. A better attempt to replicate these functions can be seen in the design of the Endolite Multiflex Ankle (Endolite; USA), a modular AJP that provides both mobility and linear moment control of the joint in the sagittal plane. Figure 3.5 displays and gives a brief description of the device.

The study Su et al. [2010] analyses whether the provision of controlled angular ankle mobility improves walking performance of bilateral TT amputees, whereby a Multiflex Ankle is attached to a baseline AFP of limited ankle mobility (Seattle Lightfoot II (Trulife USA; USA)). A retrospective analysis of the same data was later conductd conducted by the research team [Gard, 2011] ${ }^{2} \mathrm{Ad}-$ ditionally, a baseline database of the gait mechanics of $14 \mathrm{AB}$ subjects (average of 26 years, 174.2 $\mathrm{cm}, 72.3 \mathrm{~kg}, 7$ male) was created, which allowed the comparison of the amputee population (age $52.8 \pm 17.7$ years, $171.9 \pm 7.8 \mathrm{~cm}, 77.4 \pm 15.5 \mathrm{~kg}, 14$ male) to a 'normal' database [Su et al., 2010]. The summarised analysis of stance mechanics is described in Table 3.1 and Figures 3.6.
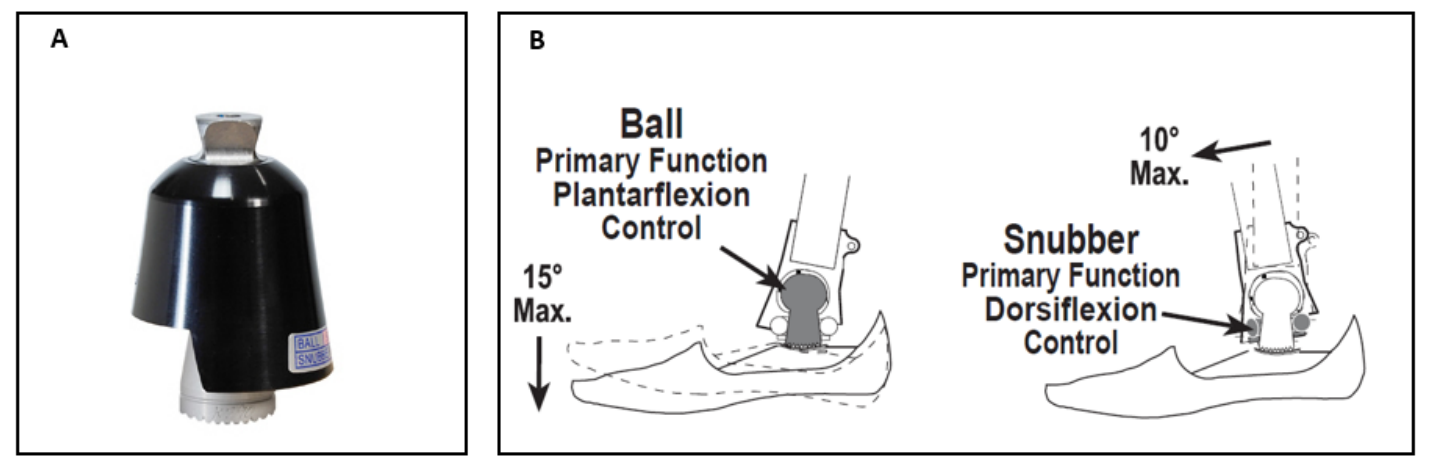

Figure 3.5: Endolite Multiflex Ankle. [Endolite, 2017]

The Multiflex Ankle (A) predominantly provides angular rotation and stiffness in the sagittal plane [Gard, 2011]. The 'ball' provides rotational limitations and moment controls during obtuse plantarflexion, whilst the 'snubber' provides the same for acute dorsi- and plantarflexion (B). Both the components control the stiffness via "combining different durometer viscoelastic elements" [Gard, 2011], and are available in a range that accommodates patient body weight, health, and activity level considerations.

\footnotetext{
${ }^{2}$ Note that both Su et al. [2010] and Gard [2011] consist of the same research teams, and that the data described in this section was originally recorded and reported in the former study. The latter study is a retrospective analysis thereof, with some adjustments to the data due to post-analysis error diagnosis.
} 
Table 3.1: The Effect of the Multiflex Ankle on Stance Mechanics. Data from Su et al. [2010].

\begin{tabular}{l|c|c|c|} 
& Able-Bodied & Baseline AFP & $\begin{array}{c}\text { Baseline AFP } \\
\text { Multiflex Ankle }\end{array}$ \\
\hline Free Walking Velocity (m/s) & $0.81(0.19)$ & $0.91(0.27)$ & $0.92(0.29)$ \\
\hline Ankle RoM ( $\left.{ }^{\circ}\right)$ & $20.2(3.5)(B, F)$ & $12.5(3.1)(F, A B)$ & $18.7(4.9)(B)$ \\
\hline Initial Vertical GRF (BW) & $1.05(0.03)$ & $1.09(0.06)$ & $1.11(0.17)$ \\
\hline Peak CDF Moment (Nm/kg) & $1.28(0.14)(B, F)$ & $1.13(0.16)(A B)$ & $1.08(0.14)(A B)$ \\
\hline Peak CPF Moment (Nm/kg) & $0.10(0.02)(B, F)$ & $0.19(0.14)(A B)$ & $0.17(0.09)(A B)$ \\
\hline Peak Power (W/kg) & $1.26(0.38)(B, F)$ & $0.38(0.18)(F, A B)$ & $0.56(0.30)(B, A B)$ \\
\hline
\end{tabular}

The letters in parentheses indicate that there was a statistically significant $(p<0.05)$ difference in means when compared to the able-bodied $(A B)$, baseline (B) or baseline + multiflex (F) groups.

Peak-to-peak RoM of the baseline, baseline + flexion unit, and AB groups are displayed in Table 3.1, which is visually displayed in Figure $3.6(\mathrm{~A})$. The analysis of this data demonstrates that the flexion attachment improves the sagittal plane motion of the baseline AFP from $64 \%$ to $96 \%$ of the able-bodied norm [Su et al., 2010; Gard, 2011]. The graphs provide a visual breakdown of the gait cycle of the $A B$ control. In terms of describing the points of transition between phases of stance of the $A B$ group, the following is observed ${ }^{3}$ : Heel (ground) contact occurs at $0 \%$, with the foot initially angled in a range around the neutral mark ${ }^{4}$. CPF follows ground contact, terminating at footflat at about $8 \%$ of the gait cycle, at a peak plantarflexion angle for this phase. CDF then facilitates the tibial progression through neutral, until a peak dorsiflexion angle for this phase is reached at about $55 \%$. Powered plantar flexion rotates the foot through neutral and into an obtuse angle at toe-off, occuring at $65 \%$ of the gait cycle. When analysing the graphical data of the amputee subjects wearing the Multiflex, it can be seen that the angle vs \% gait cycle curve follows a similar path to that of the $\mathrm{AB}$ norms. Exceptions to this are where peak dorsiflexion and plantarflexion angles occur at delayed points (5\%) to the norm (yet still falling within 1 SD in magnitude). The graph further illustrates that whilst heelstrike for the flexion and $A B$ group has been set on the graph to initiate at $0 \%$, in terms of time it takes the former longer ( $5 \%$ of the gait cycle) to reach footflat than the latter. This also occurs during the transition to PPF.

It is also notable that baseline-flexion unit appears to have been set to a neutral angle of $+4^{\circ}$, as opposed to the $0^{\circ}$ of the $A B$ group. Whilst the research teams do not give an explicit reason for this, it could be explained by the fact that clinicians often set the devices to have a dorsiflexed toe angle during swing phase of the gait cycle. What is also evident, and is an important consideration when designing passive mechanical devices, is the fact that during PPF the amputee ankle angle does not rotate further than the neutral position into an obtuse plantarflexion angle. This can be attributed to the passive nature of the unit, which relies on external force application (body weight in this instance) to move beyond neutral position.

\footnotetext{
${ }^{3}$ Note that these values are estimated from Figure $3.6(\mathrm{~A})$ and were measured via scaling.

${ }^{4} \mathrm{Neutral}$ is defined as " $0{ }^{\circ}$ between the long axis of the tibia/fibula and the line perpendicular to the plantar aspect of the foot projected onto the sagittal plane of the tibia/fibula" [Wu et al., 2002]
} 

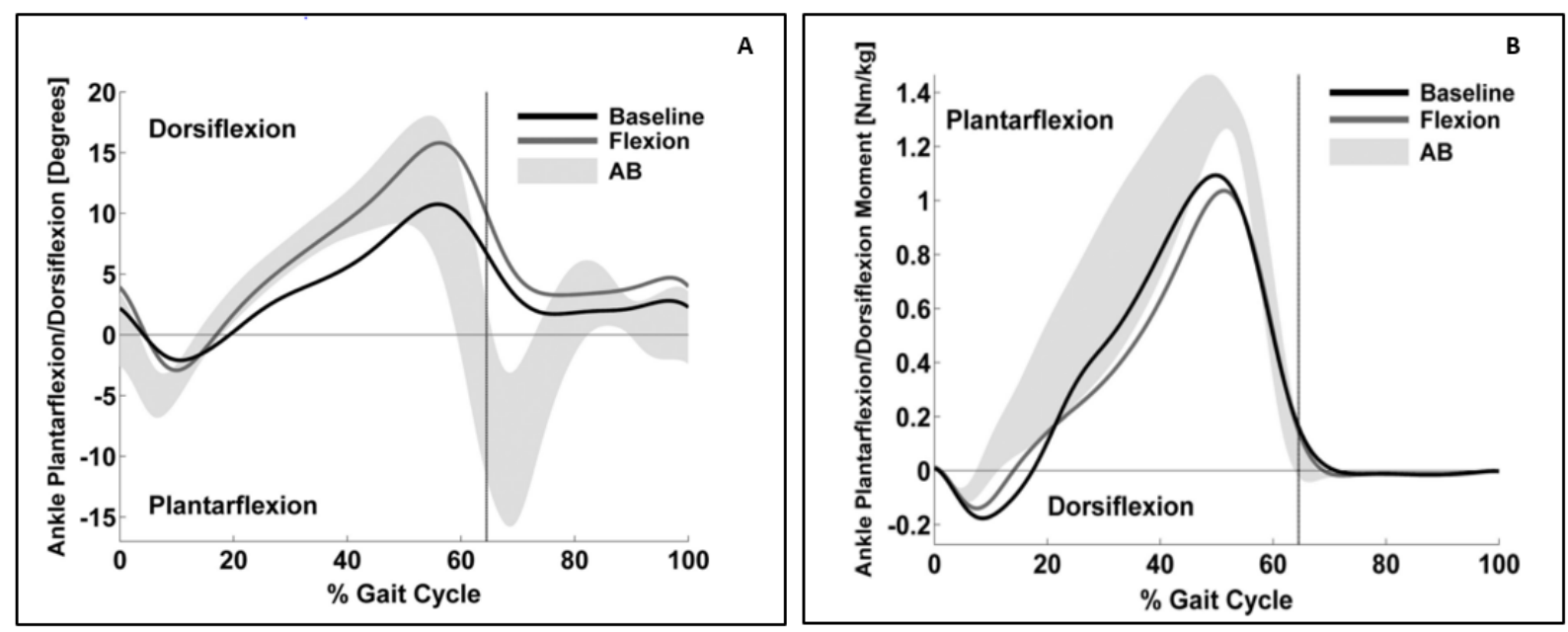

Figure 3.6: Impact of the Multiflex Ankle on Stance Mechanics. [Su et al., 2010]

This figure displays the graphical representation of stance mechanics (ankle RoM (A) and moments (B)) over the course of one stride (gait cycle). The shaded area represents the mean $\pm 1 S D$ of the able bodied (AB) group. The stance phase of the $A B$ group runs from $0 \%$ to the vertical line, which indicates the point of toe off for the $A B$ group.

The vertical GRF data shows that the reaction forces with the prosthetic configuration fall into acceptably similar ranges to that of the AB group. Also provided is graphical data (Figure 3.6 (B)) and peak summaries (Table 3.1) relating to ankle moments during the gait cycle. It is important here to reiterate that during CPF the dorsiflexor muscles apply eccentric muscle force to control transition to foot flat; during CDF the plantarflexors apply eccentric muscle force to control tibial progression; and during PPF the planterflexors undergo concentric contraction in order to provide power for toe-off [Brockett and Chapman, 2016]. The graphical representation of the data in Su et al. [2010], seen in Figure 3.1 (B), confirms the characterisation of dorsi- and plantarflexor function by Brockett and Chapman [2016]. The AB control normal curve clearly displays the transition from dorsiflexion moments to plantarflexion moments at points that occur concurrent to thes phase transitions. Likewise the moment transition points of the baseline and baseline + flexion unit group occur at their corresponding phase transitions, albeit delayed relative to the $A B$ control group. Visual analysis of Figure 3.1 (B) reveals that during the CPF phase, the mean applied dorsiflexion moments of the two amputee groups are consistently almost double that of the $A B$ group, despite displaying a corresponding ankle rotation about equal to that of the $A B$ group. This reveals that the pseudo-dorsiflexors of the unit is generating double the resistance to CPF than that of the $A B$ group. This increase in resistance could be the cause of the delayed termination of CPF discussed previously. During CDF the amputee groups mean applied plantarflexion moments are consistently $85 \%$ of the $A B$ group, indicating that the Multiflex is under-controlling the deceleration of tibial progression. Important to note is that the addition of the flexion unit decreases the peak moments when compared to the baseline group, although Su et al. [2010] notes that the differences are not statistically significant. As the peak GRF force of the AB group was statistically similar to the amputee groups, Su et al. [2010] attributed the decrease in peak moment to the 
prevention of forward shifting of the moment arm. This is due to the reduction in ankle stiffness during mid to late stance that results when the flexion unit is added.

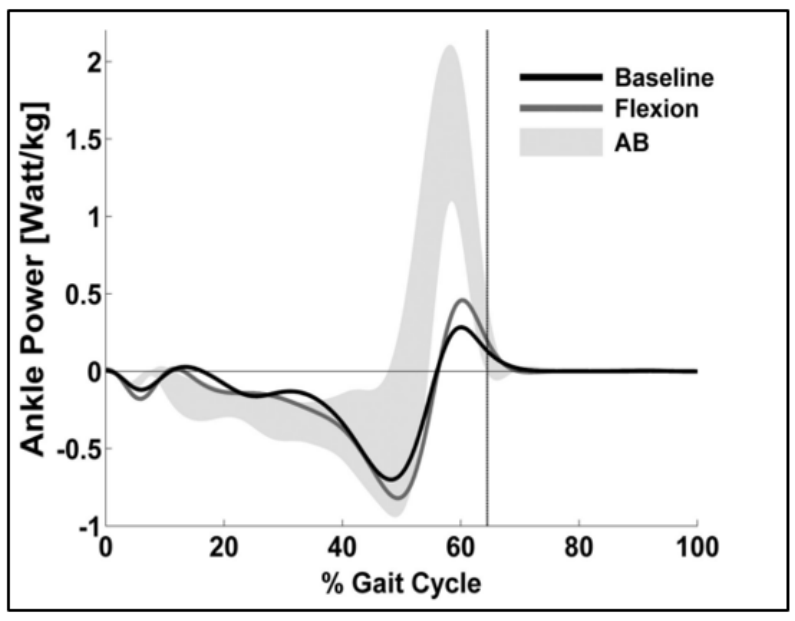

Figure 3.7: Impact of the Multiflex Ankle on Power. [Su et al., 2010].

The shaded area represents the $1 S D$ pattern of the $A B$ group. The stance phase of the $A B$ group runs from $0 \%$ to the vertical line. Negative power gradient indicates eccentric contraction of lower leg musculature, whilst positive power gradient indicates concentric contraction.

Ankle power data provides clarity as to when the musculature is absorbing (eccentric contraction = negative power gradient) or providing power (concentric contraction = positive power gradient) during the stance phase [Alexander and McNeill, 1984]. As such, Figure 3.7 shows that the CPF and CDF phases of the $\mathrm{AB}$ group are largely characterised by eccentric contraction of the dorsiflexor musculature and plantarflexor musculature respectively, whilst PPF is characterised by concentric contraction of these same muscles. Brockett and Chapman [2016] posit that maximum negative joint power occurs at the transition from CDF to PPF (45-50\% of the gait cycle), and a maximum positive joint power at about midway throught PPF (50-55\%). This is supported by the graphical representation of ankle power in the AB group in Su et al. [2010] (see Figure 3.7 and Table 3.1). One can also observe how the passive Multiflex fails to replicate these power characteristics. Thus, it is acknowledged that only the addition of moment actuators during PPF would be able to replicate the power generation of natural mechanics. This would also coincide with an obtuse plantar flexion angle during toe-off [Palmer, 1999].

Qualitative data collected from the subjects in Su et al. [2010] via a questionnaire indicates that the Multiflex provided better shock absorption and mobility during walking, and eases stair ambulation. Yet, the participants also expressed that they felt as though they might fall backwards during standing, indicating that the Multiflex results in a decrease in standing balance control. In contrast, the participants stated that the baseline unit has a rigid forefoot that is difficult to roll-over, and that it requires more effort to walk when using it. However, the participants did also indicate that they experience more standing stability without the Multiflex attachment. 


\section{Chapter 4}

\section{Theoretical Definition of Ankle Joint Mechanisms}

\subsection{Defining the Mechanics of the Ankle in Terms of Simple Mechan- ical Elements}

Palmer [1999] characterises the human ankle function during the stance phase of walking, limited to sagittal plane motion, and is specifically useful to prosthetic ankle design due to the fact that it quantifies the ankle as a function of net inputs and outputs. The study avoids identifying and characterising individual muscle, tendon or bone structure, but rather simplifies the sum of their functions into kinetic and kinematic results, operating around a central pivot point (the ankle). In this sense, it removes the requirement to directly replicate ankle input mechanisms, but rather to replicate that of the net output result. Further, the characterisation is classified into sections corresponding to the three phases of the stance previously identified (CPF, CDF and PPF), and made recommendations as to the reproduction of these dynamics in terms of simple mechanical elements based on the relationships that were observed ${ }^{1}$ (see Figure 4.1).

Analysis of the CPF phase by Palmer [1999] proves a trend of significant simple linear regression ${ }^{2}$ of increasing moment with increasing angle, as shown in Figure 4.1. Thus, it is concluded that the ankle can be modelled as a linear spring during CPF. This corresponds with the theory that the primary function of the dorsiflexor musculature during CPF is to reduce impact losses at FF, via eccentric contraction, whilst still minimising energy expenditure Alexander and McNeill [1984]. Further, the power was observed to be consistently negative, also indicating that the phase can be mimicked using passive elements. The specifics of the mechanical system implemented would depend on subject demographics.

\footnotetext{
${ }^{1}$ It is important to note that inter-participant analysis was not conducted, due to large variability in subject age, mass, height etc. Therefore statistical analysis across the sample population is not available. The study instead focused on in-depth intra-participant analysis of sagittal plane ankle mechanics, and statistical analysis thereof.

${ }^{2}$ Simple linear regression is a statistical term used to describe a straight line relationship between an independent and dependant variable. It thus describes a linear dependence of one variable on the other.
} 
Analysis of the CDF phase indicates that simple linear regression could not adequately charac-
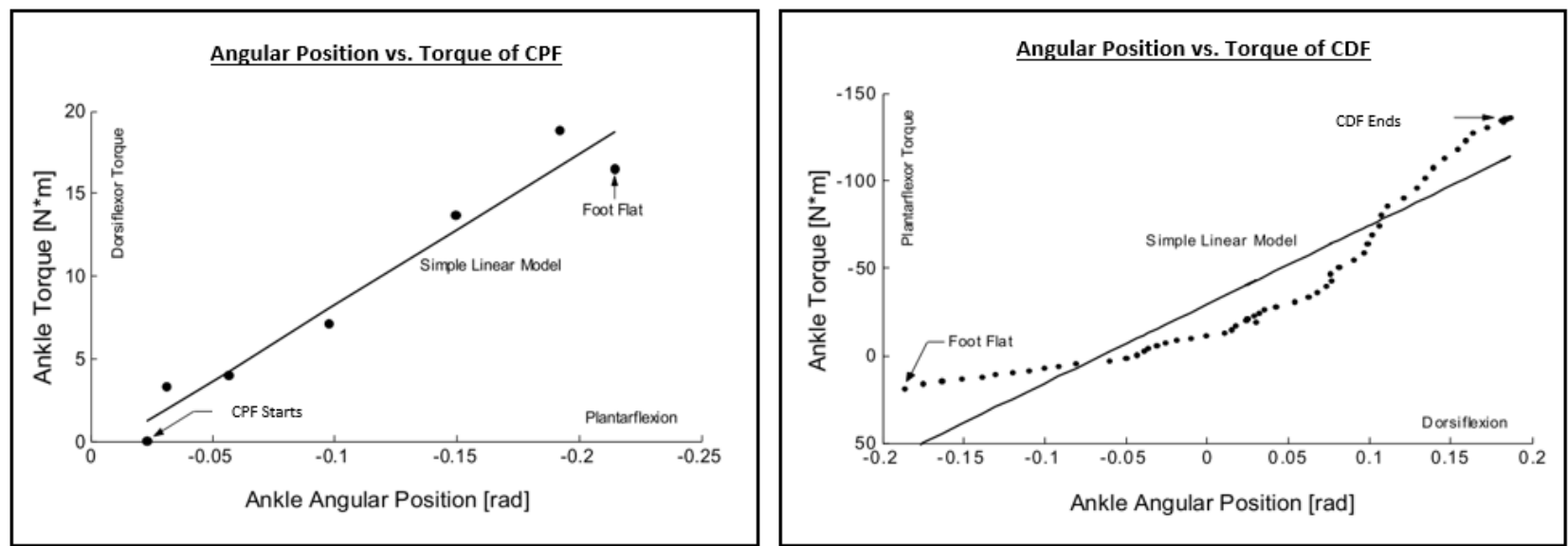

Figure 4.1: Ankle Angular Position vs moment Characterisation. [Palmer, 1999]

These graphs display an example of the relationship between ankle angle position and ankle moment for CPF (left) and CDF (right). From these relationships, Palmer [1999] characterised CPF as a linear spring $\left(p<0.001 ; r^{2}=0.95\right)$, and CDF as a nonlinear hardening spring $\left(p<0.001 ; r^{2}=0.87\right)$. Note that these figures and statistical results were generated in the analysis of a single trial.

terise the moment vs displacement relationships. Rather, it can be concluded that the ankle function during CDF should be characterised as a "nonlinear, hardening, spring for all self-selected speed groups" Palmer [1999]. This is explained by how in the latter stages of natural CDF there is a marked increase in GRF magnitude, as well as a distal shift of the GRF point of application. This yields an increase in moment arm and consequentially an increase in moment, to which the plantarflexor musculature must provide an antagonist dynamic stiffness. It is also worth noting that the phase difference between local maximum plantarflexion moment (dominant during CDF) and the local maximum angular displacement (in dorsiflexion) were consistently in phase with each other, further supporting the conclusion that the phase is dominated by springlike behaviour. Also observable is that the power during this phase was predominantly negative and could thus be modelled using passive elements.

Analysis of PPF reveals that this phase is characterised by positive power and, even in a case of $100 \%$ energy return from the passive systems used to replicate CPF and CDF, active addition of input energy is required to fully replicate the mechanics. As such, the recommendation is that the phase be characterised by "an active moment actuator for all self-selected walking speeds" [Palmer, 1999]. Thus Palmer [1999] provides an indication that stance mechanics can be replicated with the use of simple mechanical elements such as springs and moment actuators.

\subsection{Core Design Principles}

Baseline, DER and the CESR AFP's allow for zero relative ankle joint mobility, and resort to a cushioned or spring based heel as an impact absorption mechanism. Further, the immobilised 
ankle forces an unnatural tibial rollover during initial stance, with amputees often resorting to flat foot ground contact. AFP design trends that attempt to mimic the CPF of natural stance by providing a loosened joint, such as that of the mechanics of the Single Axis, have failed to do so due to the allowance of uncontrolled plantarflexion. Thus the progression to footflat is characterised as abrupt and unstable. Whilst basic AFP's such as the SACH poorly attempt to ease forefoot rollover during late stance via soft foam forefoot regions, an improvement in design thereof is the incorporation of the flexible keels in DER's. This design principle, based on replicating the natural CDF mechanism of acute controlled dorsiflexion while in footflat, would work best when working in tandem with an accurate replication of CPF.

Therefore, it is believed that by combining the acute controlled dorsiflexion design principle with a controlled version of the ankle joint mobility seen in the Single Axis, improved replication of the stance phase mechanics could be achieved. Key to such a design would be the allowance of controlled tibial-foot relative motion, and would thus differ significantly to design principles seen in the CESR. The Multiflex Ankle has shown significant improvements in this regard. Section 3.5 discussed how there is a marked increase in both angular and moment replications of natural stance mechanics when using the pseudo-ankle as an attachment to a DER.

Thus, this project will investigate the option of improving stance mechanics of baseline AFP's by the design of an AJP that operates on a principle of controlled relative ankle joint rotation, using only simple mechanical elements. ${ }^{3}$

\footnotetext{
${ }^{3}$ For the scope of this study, the term "simple mechanical elements" is used to loosely define elements that are non-electronic, simple to maintain and are ideally stock parts or at can at least be mass produced. Examples of simple mechanical elements considered in this study include a coil spring and an M12 nut and bolt set.
} 


\section{Chapter 5}

\section{Project Design}

\subsection{Hypothesis}

The research for this project has revealed that AFP's available to the mid- to low-income transtibial amputee population of South Africa are failing to replicate natural stance mechanics that occur during the level walking gait cycle. The AFP's that fall into this category have minimal to no ankle mobility, relying instead on stiff tibial roll-over shapes when the foot is in contact with the ground. This design principle in AFP's has been shown to yield poor gait mechanics and increased metabolic cost, the cause of which has been theorised to be poor functional replication of ankle joint mechanisms.

The hypothesis of this project is thus that the stance mechanics of TT amputees can be improved via the restoration of ankle joint mechanisms with a spring based AJP design attached superiorly to a baseline AFP. The baseline AFP selected for this study is a SACH-Dynamic 1D10 (Otto Bock; Germany)).

\subsection{Study Aims}

The aim of this study is thus to produce a low-cost modular AJP that restores natural ankle mechanics by replicating the function of plantar- and dorsi-flexor musculature, namely: (1) mobility in the sagittal plane around an ankle pivot; and (2) ankle joint stiffness (the relationship between ankle joint moment and angular rotation thereof), via the use of springs based design. The intention is for the AJP to serve as a sub-system that interfaces between a baseline AFP and the socket/pseudo-tibia of a TT amputees prosthetic. These three sub-systems will thus form a complete TT prosthetic system. 


\subsection{Objectives}

The objectives of this project are to:

1. Identify areas of AFP functionality that need to be improved on in low-income amputee gait and theoretically quantify the ankle joint mechanics of human stance phase during normal level walking gait, based on comparative studies of literature databases (split between Part I and II).

2. Design and develop a spring based AJP that replicates the stance mechanics specified as an output of Objective 1. The AJP must be able to integrate with a baseline AFP (Part II).

3. Perform kinetic and kinematic analysis of the AJP when in an AFP system, via pseudo-stance phase replication of TT gait using able bodied (AB) participants and pseudo-prostheses (Part III). This study will allow for the quantification of the improvement in ankle joint mechanics that the AJP provides when operating with a baseline AFP (Otto Bock 1D10).

\subsection{Scope}

The scope of this project focuses on the design of a low-cost spring-based AJP, based on ablebodied stance mechanics sourced from literature, and the analysis of the ankle joint mechanisms evident when the AJP is attached to a baseline AFP (Otto Bock 1D10, a SACH-Dynamic foot). The impact of the AJP on the baseline foot will be quantified via the simulation of trans-tibial amputee stance mechanics, using three AB participants and a custom designed pseudo-prosthesis. The study will run a comparative control test with a deactivated AJP in the pseudo-prosthesis system, to which the impact of the activated AJP can be relatively quantified in the experimental test.

The rationale for the simulation of the TT amputee gait is based on ethical implications of involving amputee participants prior to the validation of the mechanical, functional and safety performance of the device. Thus these metrics must first be validated in a simulated study. Yet the implication of this simulation methodology is that, in terms of the devices impact on actual amputee stance mechanics, it renders the results clinically insignificant. Rather, the outcomes of this study are used as indicators of potential success of a future clinical study on TT amputee participants. Therefore the experimental scope of this study includes only the analysis of the mechanical, functional and safety performance of the spring-based AJP design relative to that of a baseline AFP.

\subsection{Research and Design Limitations}

The results of this study will serve as validation of the functional impact that the AJP has on the stance mechanics of a baseline AFP. The findings will be used to provide recommendations for a subsequent design iteration. Based on these findings, future studies will be able to conduct a clinical analysis (using both quantitative and qualitative metrics) of the impact that a modified device 
has on stance mechanics of trans-tibial amputee participants.

It must also be stated that the dataset generated in the comparative baseline AFP test (i.e. with the deactivated AJP) cannot serve as quantification of baseline AFP mechanics, due to the numerous variables associated with the simulation methodology. This comparative study only serves as a control test to which the impact that the AJP has on the pseudo-prosthesis system can be relatively quantified. As only the state of the AJP is varied (activation/deactivation), this is deemed an appropriate methodology for this aim, yet the net results of baseline AFP mechanics must be seen in isolation from the reality of TT amputee walking gait cycles.

Furthermore, the research and design criteria is focused on level walking gait cycles, which largely limits ankle joint mechanics to the sagittal plane. These are idealised scenarios which do not take into account the living conditions of low-income amputees as it has been shown that metabolic cost of amputees increases when they attempt to ambulate on uneven and inclined terrain. However, this study has not broadened its focus to include the effects of these variables on TT amputee gait. Rather, the study has focused on gait characteristics on flat even surfaces, in an attempt to exclude the variability of terrain on stance mechanics. In this way the limitations serve as a baseline on which the first iteration of the AJP can be designed. 


\section{Part II}

\section{The Design of the Ankle-Joint Prosthetic}




\section{Chapter 6}

\section{Design Requirements}

\section{Overview}

The purpose of this study is to design an AJP that improves the stance mechanics of baseline AFP's (i.e. SACH/SACH-Dynamic feet or generic designs thereof). Part I conducted a review of the mechanics of the stance phase of natural gait, and concluded that common baseline AFP's are failing to replicate these natural functions. Therefore, this chapter seeks to extend on the research summarised in Part I and simplifying its findings into definitive design requirements and specifications, serving as the target criteria deemed necessary for the success of this product. Further, this chapter extends this research into the delivery of a final concept design that will meet the requirements and specifications outlined here.

As such, this chapter is divided into two sections. Section 6.1 will clarify the general and functional requirements of the device. Some of these requirements are generated from the functional analysis in Part I, such as the ability to perform controlled plantar flexion, but other requirements relate to broader ergonomic requirements, an example of which is 'ease of assembly by a clinician'. Section 6.2 will then proceed to numerically quantify the kinetic and kinematic specifications of stance mechanics, specifically that of the relationship between ankle joint angle and moment in the sagittal plane. This quantification requires the collection, sorting and comparison of the relevant data, finally concluding in the output of core specifications that can be replicated through spring-based design.

\subsection{General and Functional Requirements of a Passive Mechanical Ankle Prosthetic}

\subsubsection{Core Functional Design Objectives}

As per the research presented in Part I, the core functional design objectives (FDO's) of the design is to replicate stance mechanics, which can be divided into three phases: 
1) Control of Angular Transition into Foot Flat. As the stance phase of the gait cycle initiates, the first functional requirement of the designed prosthetic will be to replicate natural CPF. Key to this mechanism in natural mechanics is the co-ordination of the relationship between ankle joint angular rotation in the saggittal plane and moment control thereof. Thus it is theorised that the phase can be replicated using a mechanical angular-elastic relationship that mimics this joint-muscle co-ordination. Functionally this mechanism will be required to elastically absorb impact energy at heelstrike (initial ground impact) and guide the foots' transition into footflat while the tibia remains in a consistent posterior-angled position.

2) Control of Tibial Progression. Following the termination of the CPF phase is the seamless initiation of CDF, whereby an amputees' walking momentum induces the forward tibial advancement over the planted foot (from its previously poster-angled position). However, it is theorised that the elastic energy absorbed during CPF can be increasingly released here to aid the initiation and continuation of the forward tibial progression towards a neutral $\left(90^{\circ}\right)$ angle with the foot. As the knee- tibia then rotates through neutral, shifting the subjects weight anterior of the ankle, the plantar flexor musculature in an intact limb begins to decelerate forward progression for the sake of stability, preventing knee collapse. The design objective is thus to replicate this steady control with a second spring mechanism. This will serve additionally as a further method of elastic energy absorption. Natural mechanics during this phase indicate that a flat foot provides optimised stability during this phase, yet baseline AFP's (i.e. the SACH foot) are often characterised by early heel rise due to their rigid forefoot. Thus, an additional objective is to allow for an extended period of footflat during this phase.

3) Controlled Release of Stored Spring Energy during Push Off. As the tibia progresses over the stance foot, shifting the body weight anterior of the foot, dorsiflexion meets increasing resistance due to its linear relationship with ankle stiffness. Simultaneously, the contralateral limb that was previously swinging now comes into contact with the ground, marking the initiation of its own stance phase and shifting the majority of the body weight off the stance limb. This weight shift allows for the initiation of PPF in the stance limb, which in an intact limb would be characterised by powered activation of the plantar flexor musculature. However, a passive spring-based AJP would rely on the elastic return of the energy absorbed during late stage CDF. Thus the final design parameter is to allow for elastic mechanism in the prosthetic to release the energy stored during CDF, due to the reduced force application that results from the shift of weight onto the leading limb.

Outside of the focus on the stance phase, a final functional consideration is that of balance control during quiet standing. At present the design process has focused on generating a dynamic mechanical system that can passively aid the replication of the gait cycle of an intact limb. However, a secondary design consideration is how the system operates when relatively static i.e. how it reacts to uni- or bilateral quiet standing. 


\subsubsection{Ergonomic Related Considerations}

The feasibility of any AJP, AFP or prosthesis additions in a commercial market rely first on its ability to meet ergonomic considerations of amputees. Whilst this study does not intend on producing a commercialised device at this stage, the consideration of such a practicality is an important factor when assessing the ability of a device to improve the performance of the $\mathrm{SACH}$.

When attempting to replicate gait mechanics with simple mechanical elements such as springs, ergonomic considerations can potentially nullify the feasibility of a device in its concept stage. For example, net AJP size housing limitations can yield spring specifications that are infeasible to manufacture under financial constraints. Thus it is imperative that the ergonomic considerations provide rigid restrictions on the concept generation and selection procedure. The ergonomic considerations of this project are as follows:

1. For the ergonomics of use:

(a) The net size of the device has to be less than or equal to the net size of an intact ankle size of the target subject population. This is to ensure that the AJP is (i) slim for aesthetic reasons and (ii) fits within a pant leg, sock or high ankle footwear.

(b) The complete AJP must be of weight comparable to competitors (i.e. the Endolite Multiflex), to limit the energy expenditure of the amputee when using the device.

(c) The noise generation of the AJP must be negligible, to ensure that the amputee can choose to be discreet about their amputation/prosthetic if he or she prefers.

2. For the ergonomics of initial fitting:

(a) The elements within the AJP housing must be replaceable, to allow the prosthetist who fits the amputee with a prosthesis to select and insert elements that are most appropriate considering the age, weight, height and activity level of the amputee. This requirement is crucial to the modular nature of the device.

(b) For the same rationale as stated in 2 (a), the AJP housing must not change based on modular adjustments.

(c) The ease of assembly of the AJP must be maximised, and the modular adjustment of the device needs to utilise only basic toolsets.

(d) The AJP must be able to interface with a maximum range of prosthetic feet and prosthetic sockets. Further, the AJP must be removable from this assembly if required.

(e) The position of the AJP on the sagittal/transverse plane interface line must be adjustable (i.e. further anterior or posterior). This is to allow adjustments to balance control.

(f) The prosthetist who fits the AJP must have control over the initial plantarflexion/dorsiflexion angle of the foot relative to the tibia.

Likewise, the economic considerations must govern the design process. However as this study only entails the prototyping design for proof of hypothesis testing, the AJP designed in this study 
is not required to strictly adhere to these requirements, but must allow for it with minimal design changes in the future. The economic considerations for this design are thus simply to limit the cost price of the final prototype to less than R1 000.00 ( \pm 80 USD).

\subsection{Quantifying the Kinetic and Kinematic Requirements of an AJP}

This section describes the literature sourced quantification of ankle joint mechanics during the stance phase of natural gait, and the translation of the relevant data from these studies into definitive design specifications for the designed AFP device. The human ankle is a joint that has three planes of motion, yet Eng and Winter [1995] shows that $93 \%$ of the ankle work done during the gait cycle occurrs in the sagittal plane. As suggested by Palmer [1999], a model of the anklefoot based solely on sagittal plane mechanics should then produce a reasonable approximation of natural locomotion. For this reason, the collection of kinetic and kinematic variables will focus on sagittal plane mechanics. A kinetic and kinematic dataset of able-bodied stance mechanics during level walking on which the ankle stiffness of the AJP design can be based is thus required. A suitable dataset is found in Su et al. [2010], wherein the authors created an able-bodied dataset as a control for the assessment of the impact of flexion and torsion units have on baseline AFP's (as was discussed in Section 3.5). Further, the kinetic data is normalised in terms of weight, which creates the opportunity to input specific anthropometric variables into the dataset that outputs weight dependant design criteria.

Table 6.1: Able Bodied Stance Mechanics (sourced from Su et al. [2010]; Gard [2011]).

\begin{tabular}{l|c|} 
& $\begin{array}{c}\text { Able-Bodied Stance } \\
\text { Mechanics }\end{array}$ \\
\hline Free Walking Velocity (m/s) & 0.81 \\
\hline Ankle RoM $\left(^{\circ}\right)$ & 20.2 \\
\hline Initial Vertical GRF (BW) & 1.05 \\
\hline Peak CPF Angle $\left(^{\circ}\right)$ & $-6^{*}$ \\
\hline Peak CPF Moment (Nm/kg) & -0.10 \\
\hline CPF Stiffness (Nm/rad.kg) ${ }^{+}$ & $-0.95^{+}$ \\
\hline Peak CDF Angle $\left({ }^{\circ}\right)$ & $14^{*}$ \\
\hline Peak CDF Moment (Nm/kg) & 1.28 \\
\hline CDF Stiffness (Nm/rad.kg) & $5.24^{+}$ \\
\hline
\end{tabular}

(*) The value was approximated by analysing graphs displayed in Gard et al. [2011] and Su et al. [2010].

(†) The value was calculated in accordance with Equation 6.1.

The relevant temporal-spatial and stance mechanics variables from Su et al. [2010] are thus listed in Table 6.1. The kinematics and kinetics of the population gait is described as a normalised 
dataset, inclusive of standard deviations. Note that the variable "Ankle Stiffness" for each phase is calculated as a part of this design methodology, by the following equation:

Ankle Stiffness $(\mathrm{Nm} /(\mathrm{rad} . \mathrm{kg}))=$ Peak Phase Moment $(\mathrm{Nm} / \mathrm{kg}) /$ Peak Phase Ankle Angle $(\mathrm{rad})$

For example, the ankle stiffness of the CPF phase is calculated as:

$$
\text { CPF Ankle Stiffness }=0.1(\mathrm{Nm} / \mathrm{kg}) /-6^{\circ}=-0.95(\mathrm{Nm} /(\mathrm{rad} . \mathrm{kg}))
$$

Equation 6.1 generates an ankle stiffness output that assumes a linear relationship between relative joint angle and moment. This methodology is appropriate because CPF can be described as a linear relationship between moment and relative angular rotation (as discussed in Section 4.1). However, CDF is characterised as a nonlinear, hardening relationship [Palmer, 1999], and as such this methodology yields stiffness relationship that does not meet the non-linear characterisation. Yet, Palmer [1999] was not able to define this relationship with a usable equation, and in order to replicate the stance mechanics in a AJP design a clearly defined relationship is required. As for the first iteration of the AJP design, focus will be given to meeting only the peak moment requirements at $\pm 15^{\circ}$ of dorsiflexion via a linear relationship (via Equation 6.1). Thus the stiffness requirements (shown in Table 6.1) will form the basis for spring selection, with the CPF stiffness criteria generating the requirements for the pseudo-dorsiflexors and the CDF/PPF stiffness criteria generating the requirements the pseudo-plantarflexors.

Ideally the stiffness outcomes of the results of Su et al. [2010] would be assessed against a comparative source. Kerrigan et al. [1998], Palmer [1999] and Hansen et al. [2004] all produced such datasets. However Hansen et al. [2004] concludes that the quasi-stiffness characteristics of the human ankle varies with walking velocity, and as such any comparative analysis will be undermined. Thus it must be stressed that the stiffness derivative [Su et al., 2010] is an approximation of human ankle stiffness, and in reality cannot be expected to serve as a definitive characterisation thereof. Recommendations for the future iterations of this AJP design must therefore be the provision of materials (i.e. springs) that allow the fitting personnel to implement appropriate options via the use of a pre-defined selection methodology. This recommendation is further motivation for the design of a modular AJP and an ease of assembly and disassembly thereof. 


\section{Chapter 7}

\section{Design Process}

This chapter details the AJP design process, from concept development to the finalisation of the prototype design, and is laid out as described in Figure 7.1.

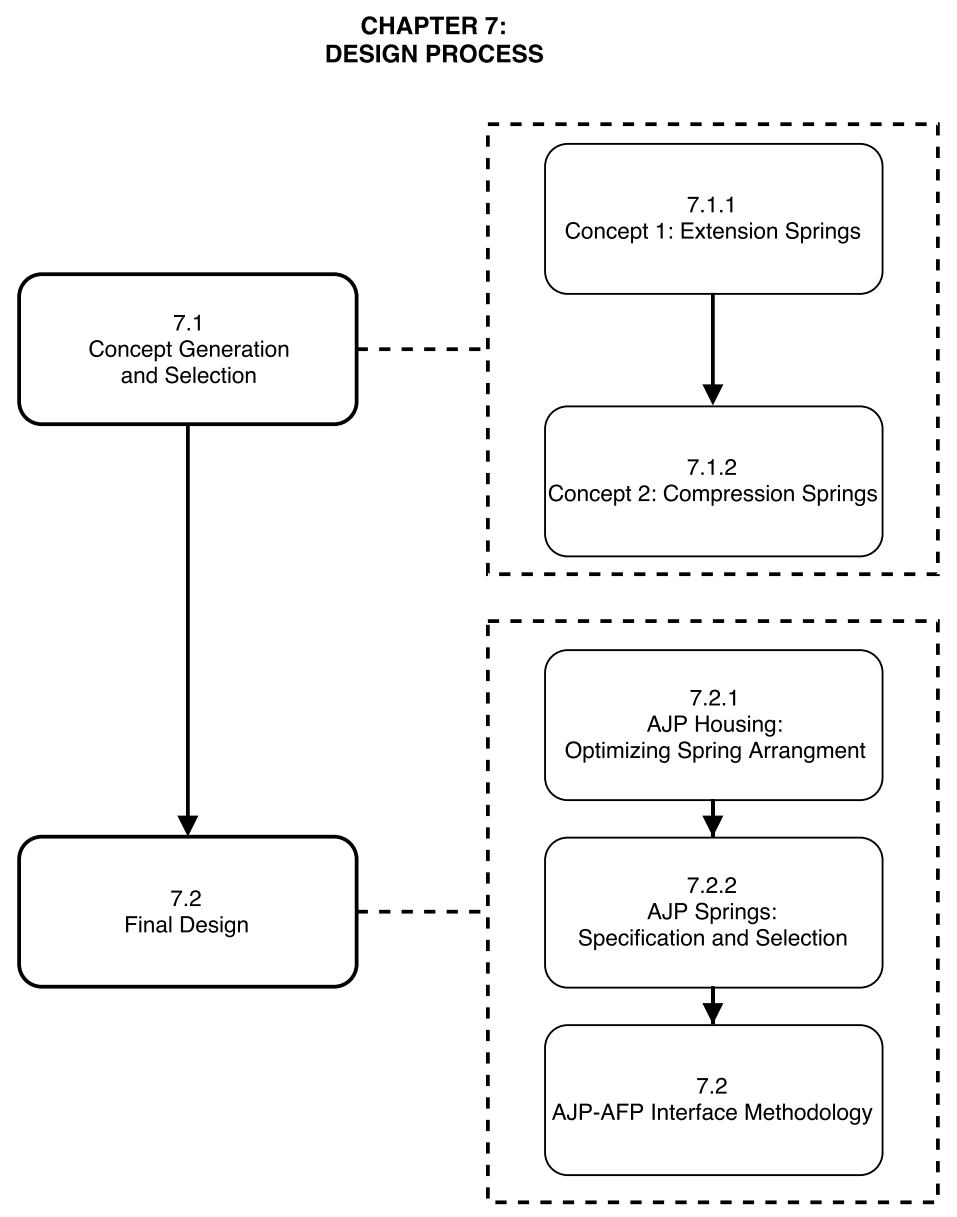

Figure 7.1: Design Process Flowchart.

This figure displays the flowchart that visually describes the summarised design process that lead to the finalisation of the AJP design. 


\subsection{Concept Development}

This section contains a linear presentation of the concept design and evaluation process. Based on the general and functional criteria presented in Section 6.1 and the stance mechanics quantified in Section 6.2, the design and evaluation of each concept considered the following factors: the identification of a spring option (i.e. torsional/extension/compression; linear/non-linear; etc.); functional implications of the spring option (i.e. spring stiffness requirements; etc.); availability and realistic assessment of springs of those specifications (i.e. is that spring a realistic option to manufacture?); safety considerations for balance control during quiet standing; and any other relevant considerations for the identified spring option.

Subsequent to the presentation and analysis of each concept, a final design concept is selected based on a variety of factors that will be discussed in this chapter. The final design and relevant information will be presented in Section 7.2 to Section 8 .

\subsubsection{Concept 1: AJP using Extension Springs}

Concept 1 was the first generation concept of this project, and differs significantly from Concept 3 and the final design. The concept intends to directly mimic plantar- and dorsi-flexor musculature via the use of extension springs. Under this principle, the springs would need to operate around a central pivot (ankle joint), as illustrated in Figure 7.2.

A critical issue that arises when using opposing extension springs is that during the extension of one spring, the opposite spring would need to undergo compression that exceeds its free length in order to accommodate the angular rotation in its direction. Natural musculature accommodates for this is more complex ways, such as lateral deflection in compression around the muscles line of action (LoA), but springs cannot replicate these compensation mechanisms. A solution to this space problem is to place the tibial hinge of each spring on a sliding rail, allowing the hinge to slide up the length of the tibia during inactivity. Figure 7.3 displays how the AJP would function when mimicking CDF. This concept uses industry standard prosthetic attachments (PA's) and procedures to attach the AJP to the pseudo-tibia and AFP. The pseudo-tibia of the device attaches to the base plate at the pivot via a nut-and-bolt arrangement. The pseudo-tibia then interfaces directly with the base of the prosthesis socket.

To induce standing equilibrium using this concept, an amputee would need to rotate forward over a flat foot as they would with an intact limb, initiating dorsiflexion which activates the pseudoplantarflexors. Yet, the risk with this balance control mechanism is that should the pseudoplantarflexors fail, either due to failure of the hinges or spring hyper-extension that yields plastic deformation, the balance control mechanism fails in its entirety and leaves the subject at risk of bodily harm. A secondary safety mechanism is required, but the incorporation thereof would complicate design, assembly and manufacturing.

As the base plate can be no larger than the approximate size of a natural ankle, the fixed hinges 


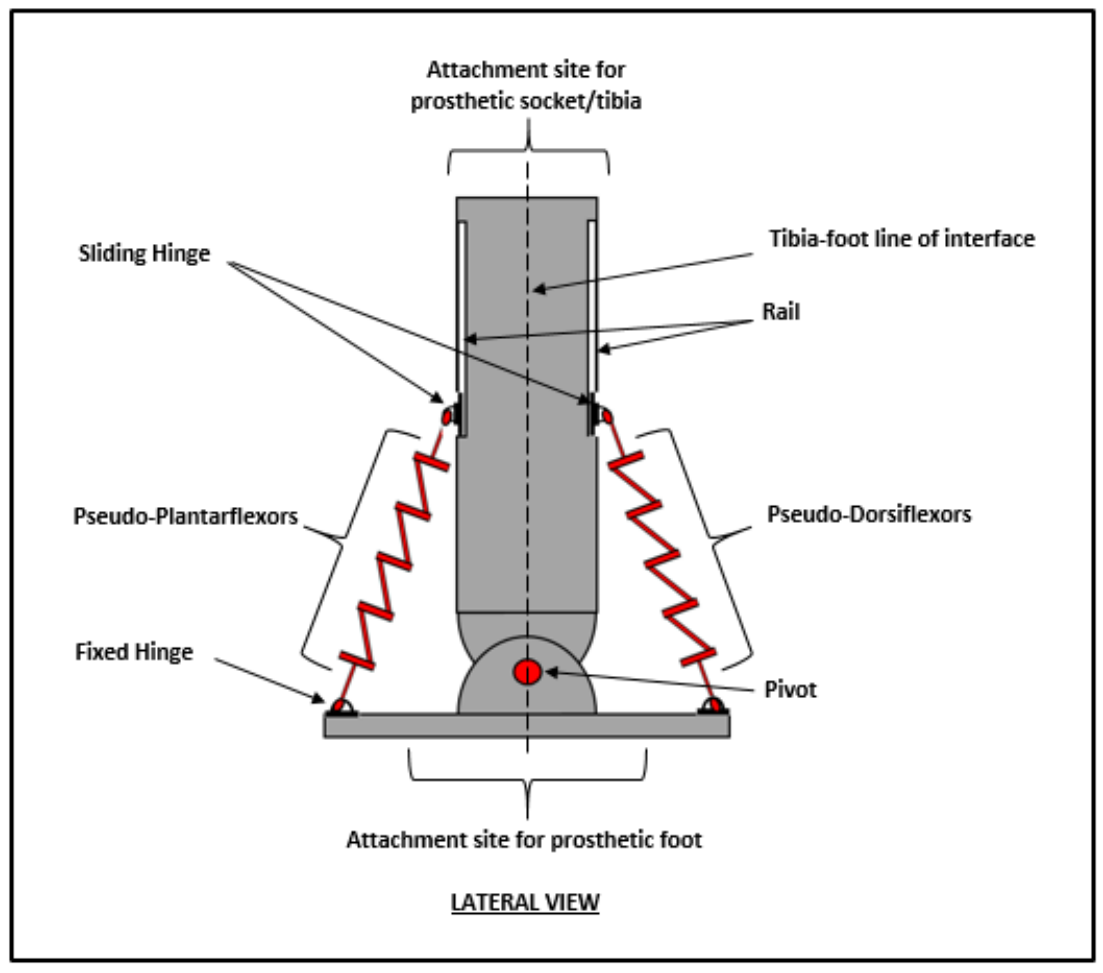

Figure 7.2: Concept 1: Using Extension Springs.

This figure displays a possible assembly arrangement of an AJP using extension springs. Note that the lateral view is displayed, and as such, the right side of the figure is anterior. In this assembly, the pivot point is a freely rotating hinge, which allows for relative rotation between the pseudo-tibia and base of the AJP. Acute dorsiflexion and acute plantarflexion is controlled by the pseudo-plantarflexor (spring) loading response, whilst obtuse plantarflexion is controlled by the pseudo-dorsiflexor loading response. The tibial hinge of each spring is able to slide up and down a rail, in order to accommodate acute angular rotation in its direction (i.e. when the spring is inactive). Attachment sites of the AJP to the pseudo-tibia/prosthetic socket and the prosthetic $(\mathrm{SACH})$ foot are indicated in the figure.

of the springs can be located $\pm 50 \mathrm{~mm}$ from the tibia-prosthetic foot interface line. Thus the line of action (LoA) of the springs creates an acute angle with the pseudo-tibia, yielding a perpendicular force application on the pseudo-tibia that is a small percentage of the loading response of the spring. Increased relative motion between the pseudo-tibia and the base plate compounds the problem, as the LoA - tibial angle becomes increasingly acute. To compensate for this, there are two design variables that can simultaneously be adjusted: (i) an increase in the LoA-tibial angle by shortening the spring and moving the sliding hinge connection point closer to the pivot; and (ii) an increase in the stiffness of the springs by adjusting the spring index (ratio between wire diameter and mean spring diameter). Yet initial calculations reveal that these design adjustments yield springs with specifications that cannot feasibly be manufactured, and compromised springs will not replicate the mechanics to an accuracy of replication required.

The dominant conclusions from this design iteration are the following: 


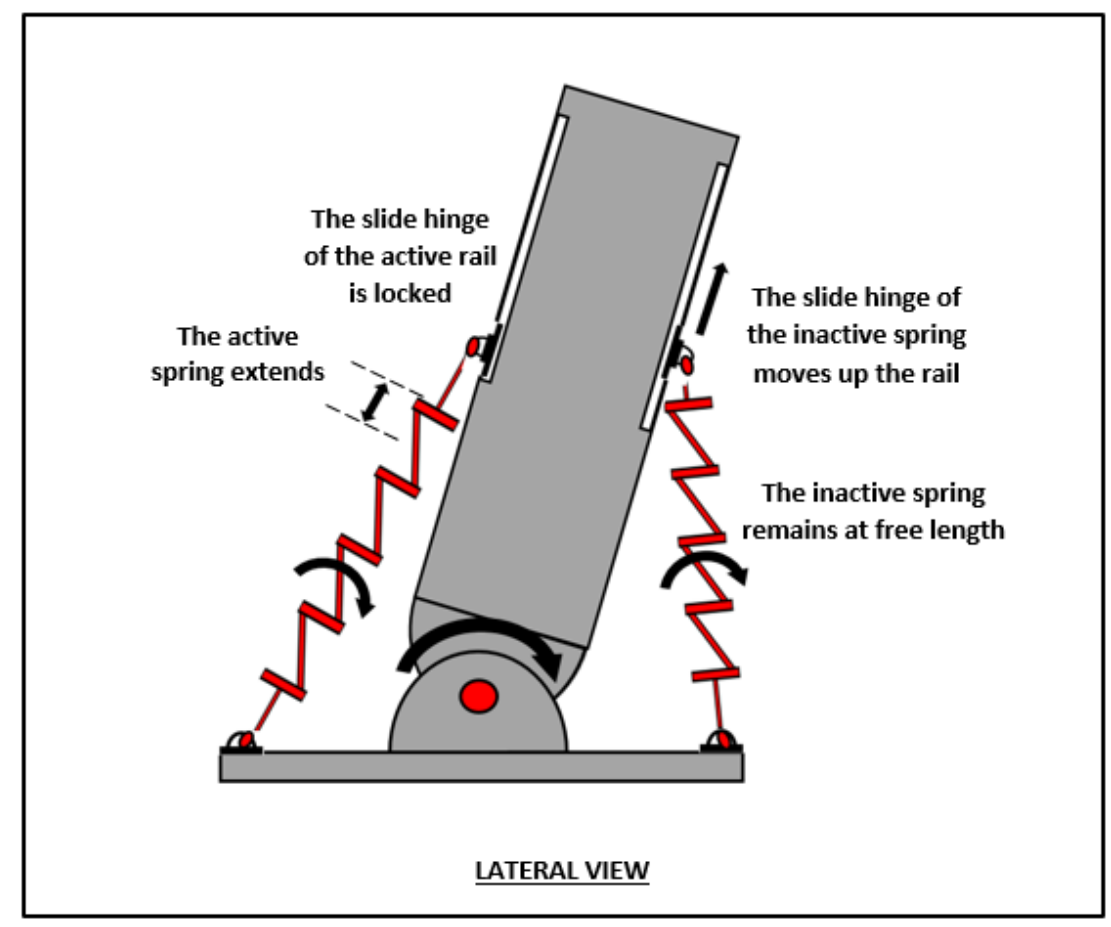

Figure 7.3: Concept 1: Function during CDF.

This figure describes how Concept 1 would function during CDF. The pseudo-plantarflexors are actively controlling forward tibial rotation during CDF, and perform this function via an extension loading response. The sliding hinge of the active spring is locked against the base of the rail. The sliding (tibial) hinge of the inactive spring, which is at free length (i.e. minimum length), slides up the rail order to accommodate acute dorsiflexion .

1. Using extension springs to replicate muscle function has intolerable risk as they do not have inherent safety mechanisms.

2. The spring specifications and assembly arrangements that are required in order to produce the necessary moment around the ankle pivot create manufacturing issues that are difficult to circumnavigate.

3. The springs are exposed, leaving them able to interface with external objects (i.e. trouser leg) and susceptible to damage and/or interrupted function.

\subsubsection{Concept 2: AJP using Compression Springs}

The criticism of Concept 1 lead to the exploration of a concept that confines the action of the pseudo-musculature into a compact housing structure. The purpose of this housing structure is to isolate and protect the spring during function. This arrangement is beneficial as it: (i) minimises interference complications with the rest of the prosthesis components; (ii) confines function to within the AJP; (iii) maximises the variability of socket and pseudo-tibia length. Here, the feasibility of using compression springs is explored. The use of compression springs immediately presents the 
need to invert the location of the pseudo-plantarflexors and pseudo-dorsiflexors, as the springs will be providing antagonist forces to CPF and CDF via compression response rather than elastic response. This is an inversion of natural musculature principles, but is deemed to be an acceptable mechanism to replicate the functions thereof. Figure 7.4 displays a possible assembly arrangement.

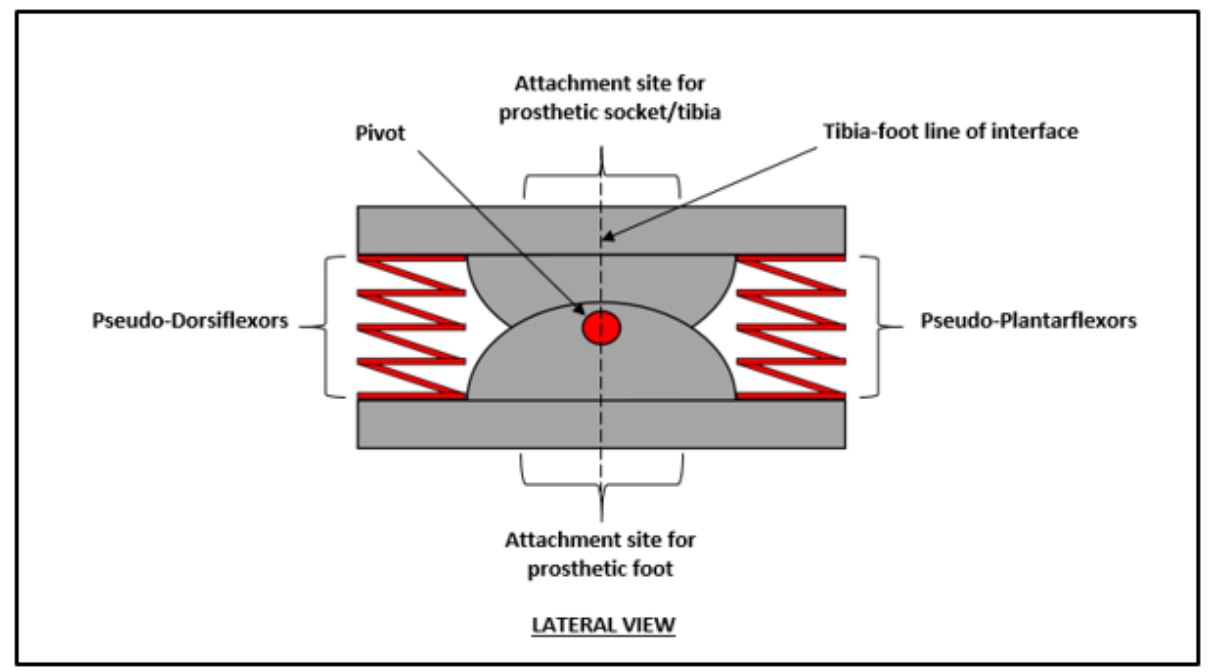

Figure 7.4: Concept 2: Using Compression Springs.

This figure displays a possible assembly arrangement of an AJP using compression springs. Note that it is viewed from the lateral view, and as such the right side of the figure is anterior. Further, not how the position of the pseudoplantarflexors and pseudo-dorsiflexors in this arrangement is an inversion of the musculature position in the human body. In this assembly, the pivot point is a freely rotating hinge, allowing for relative rotation between the upper and lower portions of the AJP housing. Acute dorsiflexion and acute plantarflexion are controlled by the pseudo-plantarflexor compression loading response, whilst obtuse plantarflexion is controlled by the pseudo-dorsiflexor compression loading response.

An advantageous characteristic of compression springs in this application is that they would provide additional structural integrity with increasing compression, as the spring itself will stiffen its rigidity up to a point of complete solidity. This characteristic of loading response can be incorporated into balance control mechanisms. Further, maximum rotational allowance in both CPF and CDF could be limited by the springs complete solidity at maximum compression (i.e. solid height). This is also advantageous in terms of minimising the risk of instability during both walking and standing, as the solid height can be used as back-up safety mechanism should the elastic response of the springs fail. In contrast, elastic failure of the extension springs yields absolute failure of stability control (as discussed in Concept 1). The other advantage of using compression springs in an AJP housing, in comparison to flat spiral springs from Concept 2, is that the location of the springs LoA can be extended to the anterior and posterior boundaries of the AJP housing, thus increasing the moment arm and consequentially the radial moment. Additionally, the number of springs in each set can be optimised by arrangement on the anterior and posterior boundaries 
of the housing, and all can be aligned perpendicular to the moment arm. Based on the ergonomic requirements listed in Section 6.1, the rough target dimensions of the AJP housing are $90 \mathrm{~mm} \mathrm{x}$ $50 \mathrm{~mm} \times 40 \mathrm{~mm}(\mathrm{LxBxH})$. This allows for a moment arm of $\pm 40 \mathrm{~mm}$ (parallel to the length (L) of the housing), a spring free length of $\pm 35 \mathrm{~mm}$ (parallel to height $(\mathrm{H})$ ), and a relationship between spring numbers and diameters that accumulate a net width of $\pm 50 \mathrm{~mm}$ (i.e. $x 2$ springs of $25 \mathrm{~mm}$ diameter, or $\mathrm{x} 3$ springs of $15 \mathrm{~mm}$ diameter, aligned parallel to the breadth (B) of the housing).

This concept does present design challenges, specifically relating to how a spring that compresses in a straight line can be arranged such that it can adequately accommodate radial displacement. Yet upon analysis of the three presented concepts, it can be concluded that the use of compression springs, and thus Concept 2 , has the highest probability of success in replicating human ankle mechanics during stance. Thus this concept forms the foundation on which the final AJP is designed, the core information of which is described in Section 7.2.

\subsection{Final Design}

This section details the design process, specifically discussing core decisions that related to the generation of a design that met the general and functional requirements. These subsections are: AJP Housing: Optimising Spring Arrangement in the AJP housing design; AJP Springs: Selection and Specification for the target prototype design; and AJP - AFP Interface Methodology that describes how the AJP will integrate with existing baseline AFP's. Refer to Figure figure 7.1 for a visual description of this process.

\subsubsection{AJP Housing: Optimising Spring Arrangement}

This subsection contains the descriptions of the core decisions relating to the optimisation of the spring arrangement within the AJP housing, namely: Balance Control Mechanisms; Adjusting Moment Arm Length Ratio; and adjustments made due to Using Linear Springs for Radial Stiffness

\section{Balance Control Mechanism}

The natural balance compensation mechanism in the body involves the anterior/posterior sway of BW, which sits anterior of the CoR, by $3-5^{\circ}$ [Winter et al., 2001]. The body alternates activation of the plantarflexors and dorsiflexors to balance sway by equalising the 'BW $x$ CoM moment' against the 'muscle force x CoP moment' based on an inverted pendulum model [Winter et al., 2001, 2003; Loram and Lakie, 2002]. Yet the concern is that the passive controllability and slower response time of spring based prostheses creates an unacceptable risk of poor balance control. It is theorised that this is a contributing cause of Multiflex Ankle instability during quiet standing (indicated by qualitative feedback from Section 3.5), and serves as motivation for the design of baseline (rigid ankle) prosthetics. 
One of the most significant design decision taken is to shift the CoR of the joint $15 \mathrm{~mm}$ posteriorly from the tibia-foot interface line (Figure 7.5). As a consequence, the CoM is shifted further forward of the CoR. This shift ensures that during quiet standing all BW is being supported by the anterior spring set. Part of the spring selection criteria therefore includes the consideration that the anterior spring set must be selected such that its compression is limited to a minimum when under a BW $x$ CoM moment (i.e. on one leg), in order to minimise the forward rotation angle when standing. Based on the approximate allowable forward rotation angle, spring deflection is limited to to $\pm 2.25 \mathrm{~mm}$ when at a moment arm of $\pm 45 \mathrm{~mm}$.

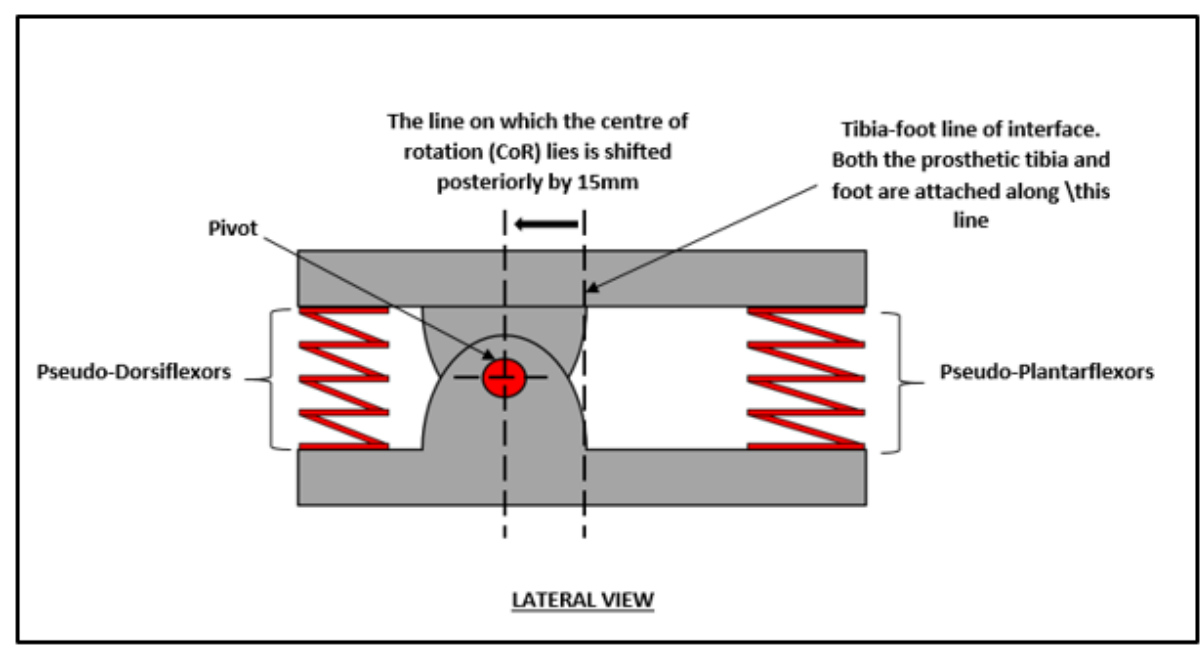

Figure 7.5: Design Solution to Improve Balance Control

This figure displays the design solution to improve balance control, the posterior shift of the CoR. This adjustment transfers more BW onto the pseudo-platnarflexors during quiet standing, introducing a balance control mechanism that intends to increase stability and remove the risk of backwards rotation of the amputee during quiet standing.

\section{Adjusting Moment Arm Length Ratio}

As well as providing a potential solution to the balance requirement, shifting the CoR posteriorly within the AJP housing also changes the ratio of anterior-to-posterior moment arm length. Given that the target stiffness of the anterior set/pseudo-plantarflexors $(5.24 \mathrm{Nm} / \mathrm{rad} . \mathrm{kg})$ is greater than the posterior set $(-0.95 \mathrm{Nm} / \mathrm{rad} . \mathrm{kg})$ by more than a factor of 5.5 , the moment arm ratio adjustment is beneficial. The pivot shift also impacts on the relative deflection of the anterior/posterior spring sets due to the adjustments to chord length at their LoA's. On the posterior side of the pivot, the pseudo-dorsiflexor spring set experiences a change in chord length of $\pm 3.93 \mathrm{~mm}$ for $\pm 10^{\circ}$ of angular rotation, while the pseudo-plantarflexors on the anterior side of the pivot experiences a $\pm 7.99 \mathrm{~mm}$ change of chord length. This is another beneficial result of the pivot shift, as it reduces the linear spring stiffness requirements of the pseudo-plantarflexors due to the increase in 
allowable deflection. Thus, by shifting the pivot point in a posterior direction, the spring stiffness requirements of the pseudo-plantarflexors is significantly reduced. This optimisation, working within the bounds of ergonomic requirements, yields the best conditions to meet target requirements when using compression springs.

\section{Using Linear Compression Springs for Radial Stiffness}

In the concept AJP housing arrangement the dual spring sets are specified to work independently of each other, and are only active during specific phases: the pseudo plantarflexors are active during acute dorsiflexion and acute plantarflexion; and the pseudo-dorsiflexors are active during obtuse plantarflexion only. A characteristic of compression springs is that they are only active under compression from free length. Thus, when the chord length is equal to or less than free length, a loading response from the springs will be induced. When the chord length is greater than free length the springs are inactive, as required. Yet a major challenge that arises during inactivity of each spring set is how to keep them aligned and located within the housing, whilst keeping the AJP housing design simple and uncomplicated to manufacture. As such, moving parts are kept to a minimum, with the only movement localised to: (i) the relative rotational movement of the two housing plates around the axle; (ii) and the localised spring deflection. The increase in chord length during inactivity of each spring set is an important factor to consider as it indicates how much 'free space' is generated, as too much space could lead to spring dislocation from the AJP housing. For the posterior and anterior side of the axle, the free space generated is $+3.93 \mathrm{~mm}$ and $+7.99 \mathrm{~mm}$ respectively. Figure 7.6 displays the design solutions that are described.

The 'free space' exposure of the posterior spring set is accommodated for with a $3.0 \mathrm{~mm}$ raised lip around the spring seats. At neutral position, the superior and inferior seats are in parallel, but at $\pm 10^{\circ}$ they are not. Once the pseudo-dorsiflexors are specified, the lateral flexibility thereof must therefore be assessed to ensure that: (i) the springs can deflect laterally to accommodate the relative angle between the superior and inferior spring seats; and (ii) that the lateral flexion does not impede the relative motion of the AJP housing around the axle. The same design amendment was used to try solve the 'free space' exposure of the anterior spring set, but it was found that their expected spring stiffness would severely limit lateral flexibility and would generate a grinding action between the spring and the spring seat. An appropriate solution for the anterior spring set is to secure the superior flat of the springs into the seat itself, leaving the inferior flat free. This would allow for the springs to remain secured in parallel with the superior AJP housing plate when inactive, thus rotating away from the inferior AJP housing plate. When the inferior and superior plates return to parallel and initiate active compression, each spring in the set still makes parallel contact with both plates. When active and under compression, the springs in the set interface

with a semicircular lip on the pivot side of the inferior AJP housing plate, preventing it from sliding towards the pivot. 


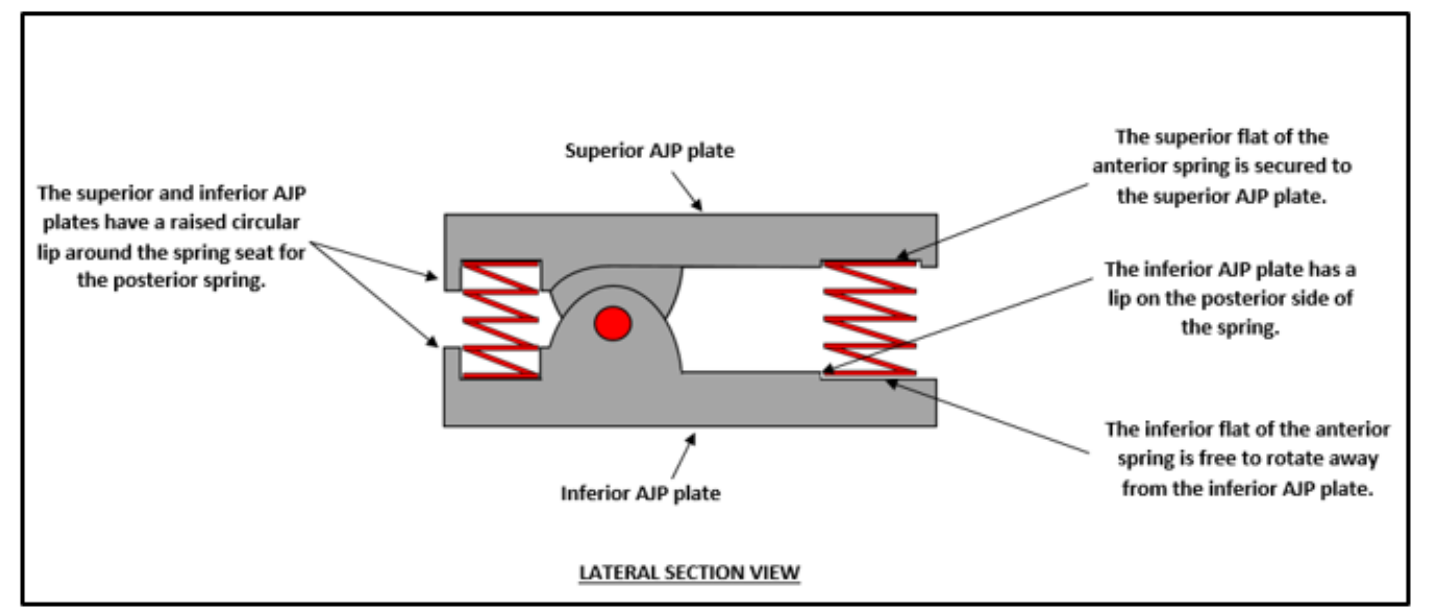

Figure 7.6: Design Solution to Accommodate for Compression Springs in Angular Rotation.

This figure displays the design solution that allows for the use of compression springs in a device that undergoes angular rotation. In this solution, the posterior springs are located within raised spring seats. During inactivity, the angle between the inferior and superior AJP plates increases on the posterior side of the pivot, creating free space around the springs. This design solution keeps the springs within the housing, but requires the springs to have some lateral flexibility in order to create an arc between the inferior and superior seats. However, the anterior springs are significantly less laterally flexible than the former due its higher stiffness requirements, and thus cannot form an arc. Thus the superior flat of the springs are secured to the superior AJP plate, and will remain perpendicular to it at all times during inactivity. During active compression, the inferior flat of the spring will lock against an inside lip on the inferior AJP plate, preventing the spring from buckling.

\subsubsection{AJP Springs: Specification and Selection}

This subsection describes the spring selection process that lead to both the final spring specification and the internal AJP housing design. Note that whilst this is presented in this report as a linear process, in reality it was an iterative process. This is due to the number of unknown spring variables (e.g. spring length, wire diameter, outer diameter, material, number of coils, and other variables) that need to be simultaneously adjusted in conjunction with internal AJP housing arrangement (e.g. moment arm length). The relationship between these variables needs to be iteratively adjusted until a theoretically viable final specification that meets the target ankle stiffness requirements is produced. Further, once the spring dimensions have been theoretically specified they must be sourced from the catalogue, and it is not guaranteed that they will be available. Thus an approximation of this iterative process is displayed in Figure 7.7. 

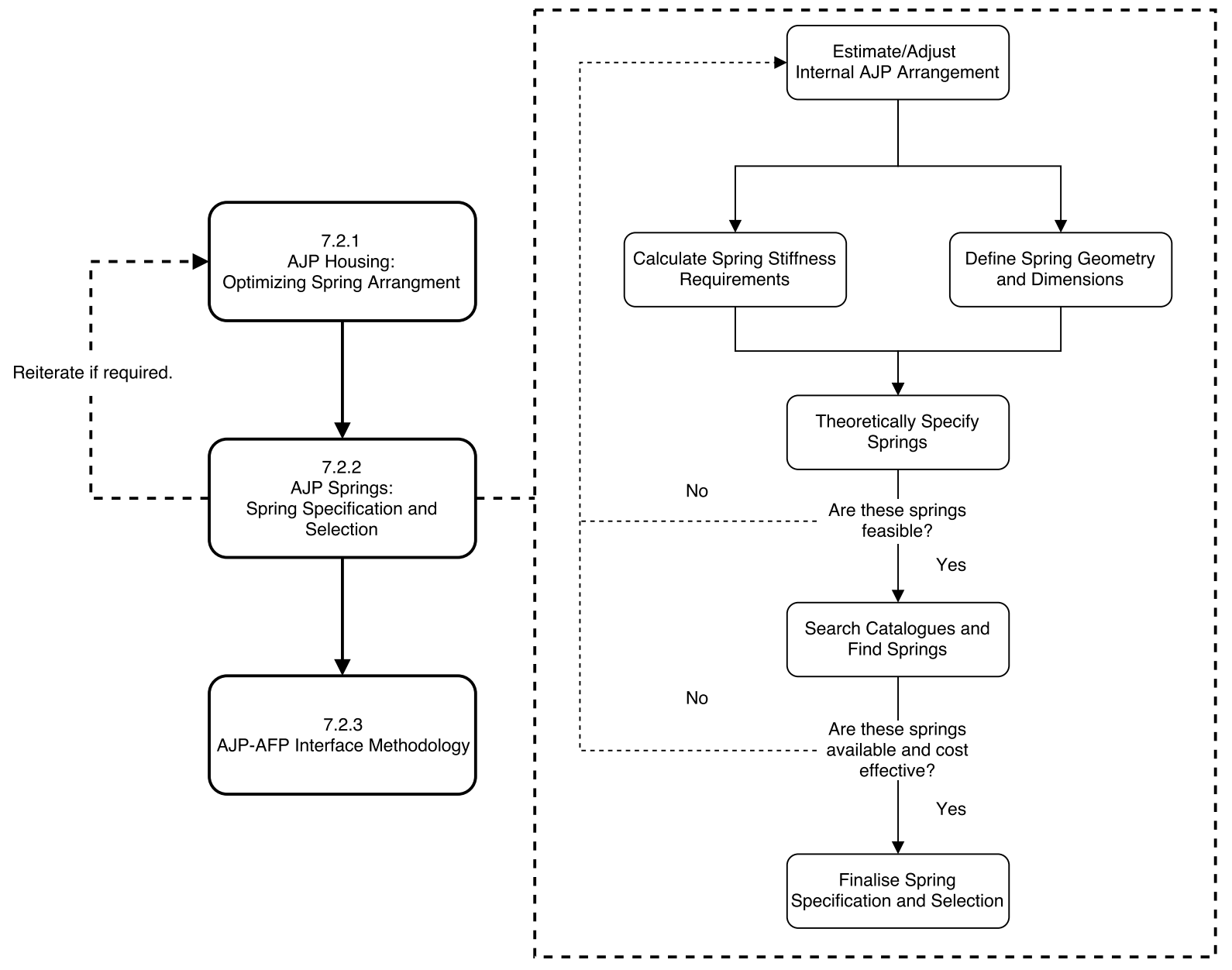

Figure 7.7: Flowchart of the Iterative Spring Specification and Selection Procedure.

This figure displays the flowchart that visually describes the iterative nature of the spring selection and specification procedure. This procedure uses general AJP housing design dimensions from Section 7.2.1 as a start-point to estimate the internal AJP arrangement and design. It further attempts to specify and select springs that meet the functional requirements, yet are also feasible to manufacture and are available from local suppliers. This flowchart also displays the context of when this process occurs in relation to the high level design procedure.

As such, the design variables and options under this subsection are presented in a linear fashion that approximates how the design process would follow if the internal AJP housing arrangement was predefined and set as an non-adjustable design factor. Yet in reality the final internal housing arrangement was the the last design variable to be finalised as it is dictated by the dimensions of the springs selected from a catalogue based on stiffness criteria.

\section{Input Criteria}

A simplified version of the final AJP internal arrangement is displayed in Figure 7.8, inclusive of the dimensions relating to the spring specification inputs. The principle purpose of displaying these dimensions is to highlight the compression that each of the pseudo-plantarflexors (left) and 
pseudo-dorsiflexors (right) experience at $10^{\circ}$ of active rotation $(8.10 \mathrm{~mm}$ and $4.01 \mathrm{~mm}$ respectively), as well as to display the radius of rotation and linear moment arm of each springset. These dimensions are used as input variables of spring specification.

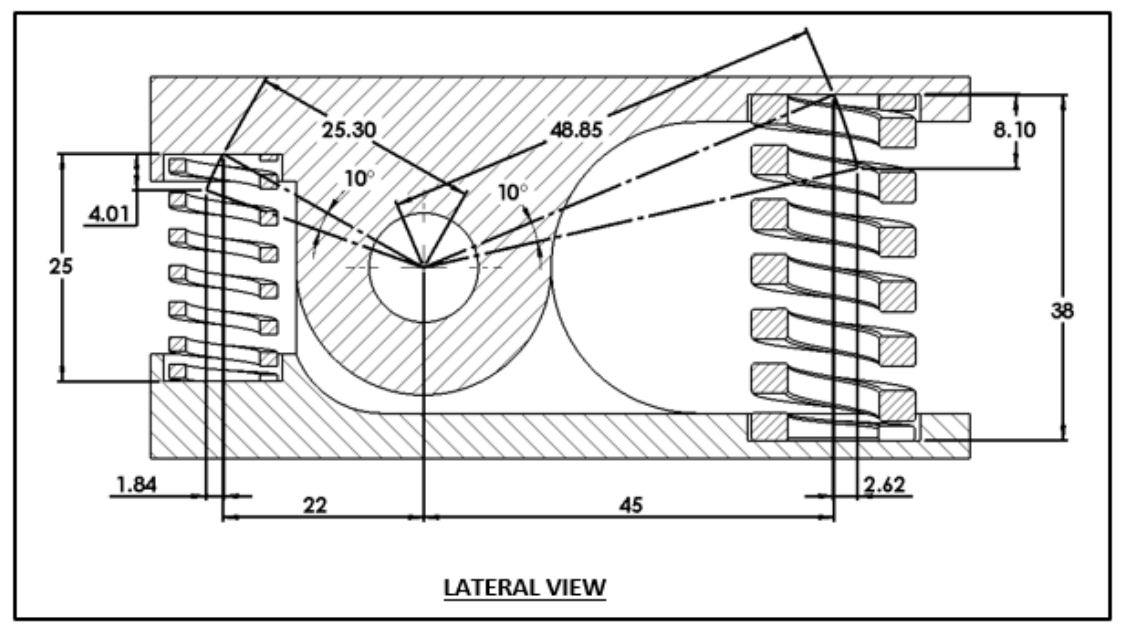

Figure 7.8: Internal AJP Housing Arrangement.

This figure displays a cross section of the AJP housing, as seen from a lateral view (anterior on the right of the image). The principle purpose of displaying this figure is to display the compression that the pseudo-plantarflexors and pseudodorsiflexors experience when under to $10^{\circ}$ of active rotation $(8.10 \mathrm{~mm}$ and $4.01 \mathrm{~mm}$ respectively). Additionally the figure displays the radius of rotation of each springset, the moment arm length when at neutral position, and the extension of moment arm when under $10^{\circ}$ of compression (2.62 $\mathrm{mm}$ and $1.84 \mathrm{~mm}$ respectively).

\section{Spring Selection}

The first compression spring option evaluated are circular wire compression springs. The limiting factor of spring stiffness in these springs is the ratio of mean coil diameter to wire diameter, termed spring index. The strength of a spring is inversely proportional to the spring index, and thus a lower spring index yields a stiffer spring. Yet the reduction of the spring index ratio is limited by manufacturing feasibility. Spring manufacturers commonly produce springs with an index of $6-12$, as springs in this range are cost effective to produce and also yield a long spring life. Yet springs with an index as low as 4 can be custom manufactured, albeit at a higher cost due to the associated manufacturing complexities. However, the spring design process (detailed in Appendix A) finds that wire compression springs fail to produce springs that are feasible to manufacture whilst still meeting the specifications required to reproduce the target pseudo-ankle mechanics.

Therefore, an alternative spring option was sought that meets both these requirements. A solution was found in die springs of rectangular cross section. In comparison to a circular wire profile, a rectangular wire profile yields an increase in structural rigidity via an increase of cross sectional area for a given width. The increase in rigidity yields a decrease of the stresses in a wire 
when under a given compression, and increases the spring constant of the spring. The rectangular cross sectional profile of the spring spiral is arranged such that breadth is greater than height. Thus, the breadth maintains lateral stiffness and mitigates buckling, whilst the height reduction increases the available space for compression. Yet the cross sectional area remains equal to or greater than that of a circular profile. The net results of this profile adjustment is an increase in both stiffness and range of compressibility, thus yielding an increase in the maximum allowable load response for a given deflection. The manufacture of the springs is done in accordance with ISO 10243 ISO [2010], which dictates the standardization of die springs. Additionally, it dictates that springs be characterised into different load capacity categories assuming all factors the same with the exception of the cross sectional profile. Furthermore, the standardisation includes the prescription of a colour code to each category: light load (green); medium load (blue); heavy load (red); and extra heavy load (yellow).

A South African company (Mould and Die Solutions (Pty) Ltd) manufactures die springs locally in accordance with ISO 10243:2010 [ISO, 2010]. Further, the manufacture of the springs is also in accordance with quality control processes as stipulated by ISO 9001:2015 [ISO, 2015]. This ensures dimensional accuracy, durability and reliability. Springs were selected from their catalogues based on the requirements of the AJP design. Table 7.1 displays the specifications of the springs selected from this catalogue, an extract of which is displayed in Appendix C. Figure 7.9 displays an example of the recommended hole and shaft dimensions when locating the pseudo-plantarflexor spring in an assembly.

Table 7.1: Selected Die Spring Specifications.

\begin{tabular}{l|r|r} 
& Pseudo-Plantarflexor & Pseudo-Dorsiflexor \\
\hline Location in AJP Housing & Anterior & Posterior \\
\hline Number of Springs & 2 & 3 \\
\hline Load Category & Heavy (Red) & Heavy (Red) \\
\hline Free Length (mm) & 38 & 25 \\
\hline Wire Breadth x Height (mm x mm) & $4.0 \times 3.3$ & $2.4 \times 1.9$ \\
\hline Hole Diameter (mm) & 20 & 12.5 \\
\hline Shaft Diameter (mm) & 10 & 6.3 \\
\hline Spring Rate (N/mm) & 129.0 & 42.1 \\
\hline Long Life (20\%) Deflection (mm) & 7.6 & 5.0 \\
\hline Max (30\%) Deflection (mm) & 11.4 & 7.5 \\
\hline
\end{tabular}

\section{Theoretical Stiffness Output}

Based on the variables displayed in Figure 7.8, the approximate moment response of the AJP at $13^{\circ}$ of dorsiflexion and $6^{\circ}$ of plantarflexion is as displayed in Table 7.2. The calculations that yield these results are displayed Appendix A. 

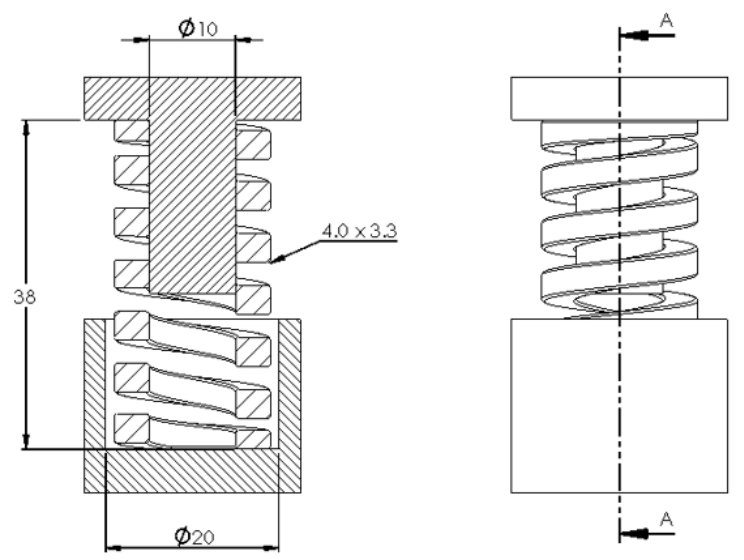

Figure 7.9: Recommended Die Spring Assembly.

This figure displays a cross section of a pseudo-plantarflexor spring when located within an example assembly, with the hole and shaft dimensions given as recommended by [ISO, 2010].

Table 7.2: Theoretical AJP Stiffness.

\begin{tabular}{|c|c|c|}
\hline & Pseudo-Plantarflexors & Pseudo-Dorsiflexors \\
\hline Spring Stiffness (N/mm) & 129.00 & 42.10 \\
\hline Expected Rotation $\left({ }^{\circ}\right)+$ & 13 & 6 \\
\hline Compression $(\mathrm{mm})$ & 10.43 & 2.33 \\
\hline Force Response (N) & 2691.60 (x2 springs) & 295.17 (x3 springs) \\
\hline Moment $(\mathrm{Nm} / \mathrm{kg})^{*}$ & 1.74 & 0.75 \\
\hline Approximate Stiffness (Nm/rad.kg)* & 7.69 (146.78\% of target) & 0.88 (92.36\% of target) \\
\hline
\end{tabular}

(†) As per the source data [Su et al., 2010; Gard, 2011])

(*) For a participant of $72.7 \mathrm{~kg}$ (as per the source data [Su et al., 2010; Gard, 2011])

\subsubsection{AJP-AFP Interface Methodology}

As indicated in the concept drawings, the AJP housing is required to interface between the pseudotibia and AFP of an amputees prosthesis. Additionally, as per the ergonomic requirements stipulated in Section 6.1, the methodology of attachment must be universal such that the device can easily integrate with common industry fitment procedure as well as to maximise the range of prosthetic feet that the device can attach to.

The most used standard prosthetic attachments (PA's) used for the interface of a pseudo-tibia and a AFP in the industry are male and female pyramid socket attachment plates. Typically the prosthetic foot has an inbuilt male pyramid socket attachment, whilst the distal end of the pseudotibia is fitted with a female socket attachment. This is the sole methodology used in the local public sector. Therefore male and female pyramid socket attachments were sourced for the AJP device, 
and attached to the AJP housing as displayed in Figure 7.10. The female is attached to the inferior plate of the housing for interface with the prosthetic foot, whilst the male is fitted to the superior plate for interface with the pseudo-tibia.

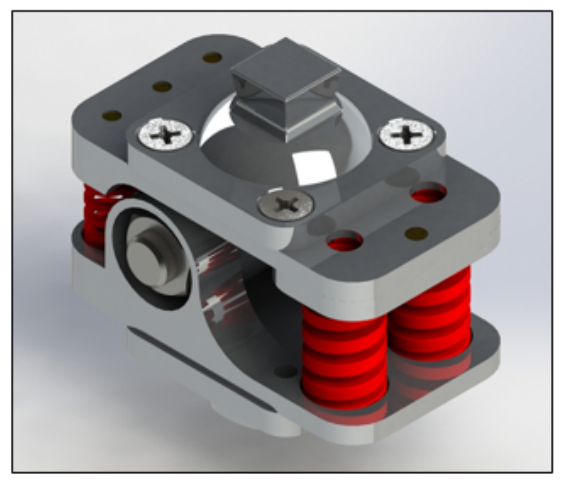

SUPERIOR/ANTERIOR VIEW

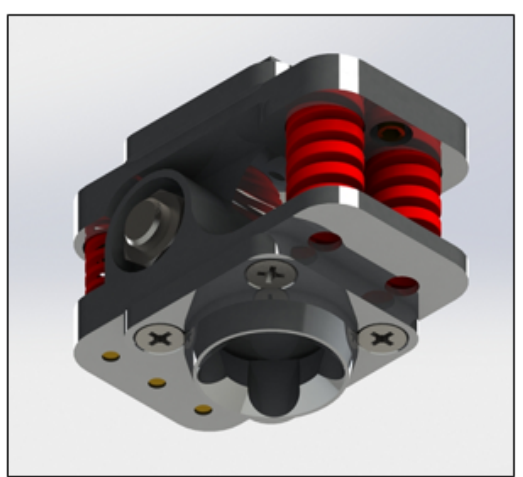

INFERIOR/ANTERIOR VIEW

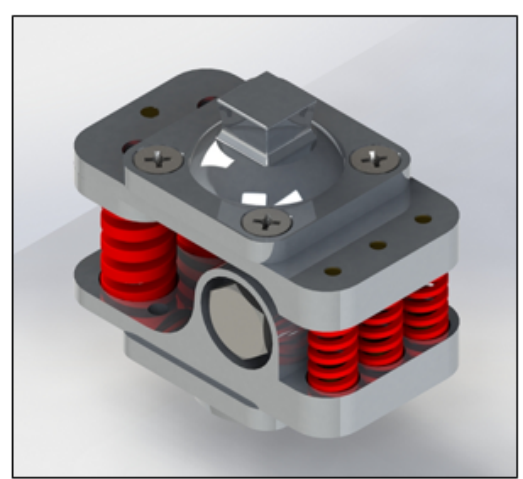

SUPERIOR/POSTERIOR VIEW

Figure 7.10: Render of Final AJP Model.

This image displays three views of a render of the final AJP model, which is created using SolidWorks 3D CAD software (SolidWorks Corp.; USA). Prominent in the superior/anterior view are the pseudo-plantarflexor springs (x2) and the superior prostheses pyramid attachment (male), whilst the inferior prosthesis pyramid attachment (female) is displayed in the inferior/anterior view. The superior/posterior view provides a perspective on the back of the AJP, in which the pseudo-dorsiflexors springs (x3) are displayed. The central axle of the device is an M12 hex head bolt. 


\section{Chapter 8}

\section{Evaluation of Final Design}

This section describes the post-design analyses of the AJP, for functiona and structural verification prior to the human trials. The key potential failure points that were identified in this design are: (a) the structural integrity and expected life cycle of the springs; (b) the stress and deflection that the AJP body will experience under peak loading conditions; and (c) the stress/deflection that the axle will experience under peak loading conditions.

A render of the final AJP model, inclusive of prosthetic attachments (PA's) and generated with SolidWorks 3D CAD software (Solidworks Corp.; USA), is displayed in Figure 7.10. For dimension information and assembly explanations, refer to the part and assembly drawings displayed in Appendix D. Figure 8.1 displays images of the final manufactured AJP device mounted on an Otto Bock 1D10 (a SACH-Dynamic foot), using only the inferior (female) PA. The foot is sized at $27 \mathrm{~cm}$ in length, in accordance with approximate foot size derived from the height and sex of an average male [Giles and Vallandigham, 1991].

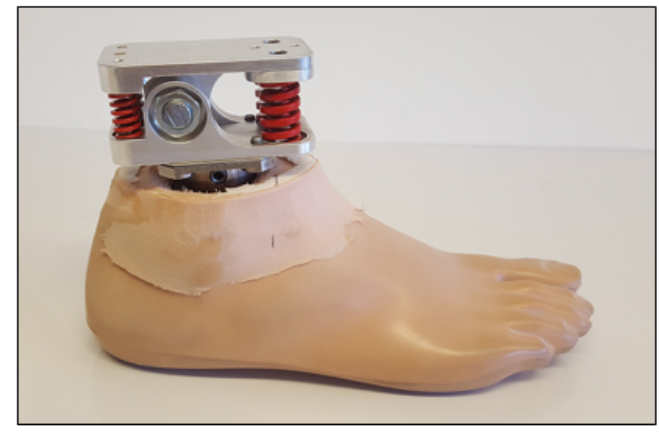

LATERAL VIEW

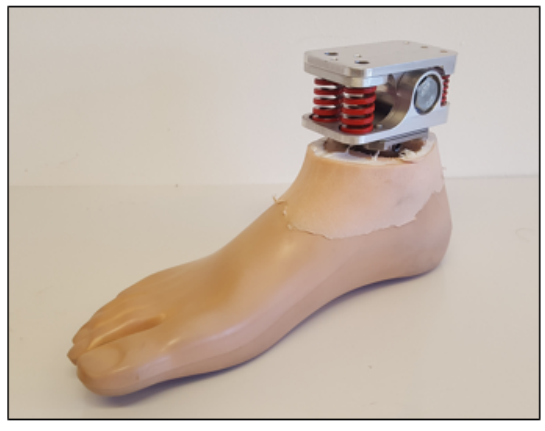

MEDIAL/ANTERIOR VIEW

Figure 8.1: Manufactured AJP Mounted on SACH Foot.

This figure displays images of the manufactured AJP as mounted on a SACH foot (27 cm, right foot). The AJP is connected to the SACH's male pyramid prosthesis attachment via a female prosthesis attachment 


\subsection{Spring Lifetime}

The quality control of the die cast springs is strictly governed by ISO 10243:2010 ISO [2010]. It states that spring catalogues must quantify spring deflections that will yield a corresponding number of life (compression) cycles. Extracts from two such a catalogues are displayed in Appendix C. The magnitude of deflection that the springs used in the prototype AJP are expected to undergo, during the normal use of the prosthesis, yields a maximum life cycles of $>3$ million cycles for both sets of springs. Under maximum expected deflections (extreme circumstances), the pseudoplantarflexor springs have an expected lifetime of $<1.5$ million (calculations displayed in Appendix C.

A reasonable estimation for the number of steps taken is 2500 steps per day per foot. Thus the pseudo-plantarflexor springs have an estimated lifetime of at least 1200 days (3 years and 4 months), whilst the pseudo-dorsiflexor springs have an estimated lifetime of 600 days (1 year and 8 months), when accounting for maximum expected spring compression during each step. Therefore maintenance plans for the AJP will specify replacement of the springs accordingly. Due to the ease of assembly/disassembly of the device, a patient can replace the springs themselves if they are provided with the replacement springs and appropriate tools (i.e. a spanner and a set of allen keys).

\subsection{Cost Breakdown and Assembly Analysis}

The cost breakdown of the prototype AJP is detailed in Table 8.1, which states that the prototype developed for experimental validation has a cost price of R595.00 ( \pm 50 USD), not inclusive of labour. The materials listed were purchased from local wholesalers, but the cost price of the prototype could be reduced if the material was brought in bulk. The Multiflex Ankle discussed in Section 3.5 has an estimated commercial price in South Africa of \pm R25 000.00, and thus the potential commercial price of the prototype AJP is well below that of its functional competitor, although this is dependant on factors not discussed in the scope of this study.

Table 8.1: Cost Breakdown of AJP Prototype.

\begin{tabular}{l|r|r}
\multicolumn{1}{c|}{ Item } & \multicolumn{1}{c|}{ Cost (Rands) } & \multicolumn{1}{|c}{ Details } \\
\hline AJP Housing Material & 342.00 & $\begin{array}{r}\text { Aluminium } 6082-\mathrm{T} 6 \\
\times 2 \text { off } 100 \times 45 \times 60 \mathrm{~mm} \text { block }\end{array}$ \\
\hline Die Springs (Anterior Set) & 117.00 & $\begin{array}{r}\times 2 \text { Heavy } \\
4.0 \times 3.3 \times 38 \mathrm{~mm}\end{array}$ \\
\hline Die Springs (Posterior Set) & 106.00 & $\begin{array}{r}\times 3 \text { Heavy } \\
2.4 \times 1.9 \times 25 \mathrm{~mm}\end{array}$ \\
\hline Nut and Bolt (Axle) & 15.00 & $\begin{array}{r}\text { Galvanised Steel } \\
\text { M12 }\end{array}$ \\
\hline Extras (screws etc.) & 15.00 & \\
\hline Total Cost per AJP & 595.00 & \\
\hline
\end{tabular}


Ease of maintenance of the device has been maximised via the use of an M12 bolt and nut (see Appendix D), thus allowing anyone with a socket wrench to disassemble the AJP housing. Disassembly of the AJP housing frees the posterior spring set, thus allowing for ease of replacement thereof. However, the prototype assembly methodology utilises an adhesive to secure the anterior spring set into its superior spring seat. This methodology, whilst appropriate for the continuation of this study, needs to be rethought if ease of maintenance is to be maximised.

Yet indications are that the aim of producing a low-cost and low-maintenance AJP has been achieved. Furthermore, the simplicity of the device has been prioritised and as such the entire AJP can be disassembled and maintained by anyone with (i) a socket wrench, (ii) a set of hex keys and (iii) a replacement set of springs.

\subsection{Finite Element Analysis of AJP Housing}

To verify the structural integrity of the AJP housing, the 3D CAD model is evaluated under peak dorsiflexion and plantarflexion loading conditions via the computational finite element analysis (FEA) simulation feature in SolidWorks. FEA is a computational methodology that is used for analysis of structural integrity by isolating key areas that are highlighted as potential failure points, and simulates the conditions that they will experience under peak load. These peak loading conditions are described in Table 8.2.

An example of the results of the stress analysis under peak dorsiflexion load conditions is displayed in Figure 8.2, which also visually displays some of the assumptions made in the simulation.

Table 8.2: Peak Load Conditions

\begin{tabular}{l|r|r} 
& \multicolumn{1}{|c|}{ Plantarflexion } & \multicolumn{1}{|c}{ Dorsiflexion } \\
\hline Angular Rotation $\left(^{\circ}\right)$ & 15 & 15 \\
\hline Spring Compression $(\mathrm{mm})$ & $3.93(16 \%)$ & $12.07(32 \%)$ \\
\hline Force Response $(\mathrm{N})$ & $752.37(\times 3$ springs $)$ & $3114.70(\times 2$ springs $)$ \\
\hline
\end{tabular}

The following assumptions are made when simulating the peak loading conditions in the FEA:

1) The AJP is manufactured from Aluminium Alloy 6082-T6, of elastic modulus $71 \mathrm{GPa}$ and yield strength $280 \mathrm{MPa}$.

2) The locking points of the male and female PA's are assumed to be a fixed geometry.

3) The spring force at peak load is assumed to act perpendicular to the spring seat faces (i.e. perpendicular to the upper and lower housing plates) in equal magnitude. Thus each spring seat of the pseudo-plantarflexors experiences $1557.35 \mathrm{~N}$ of direct force. 
4) The contact sites between components is set to bonded, making the assumption that the upper and lower AJP housing parts are bonded to the axle. Whilst this is not an accurate replication of peak loading, the assumption is based on the fact that these parts will not be able to freely move relative to each other under peak loading.

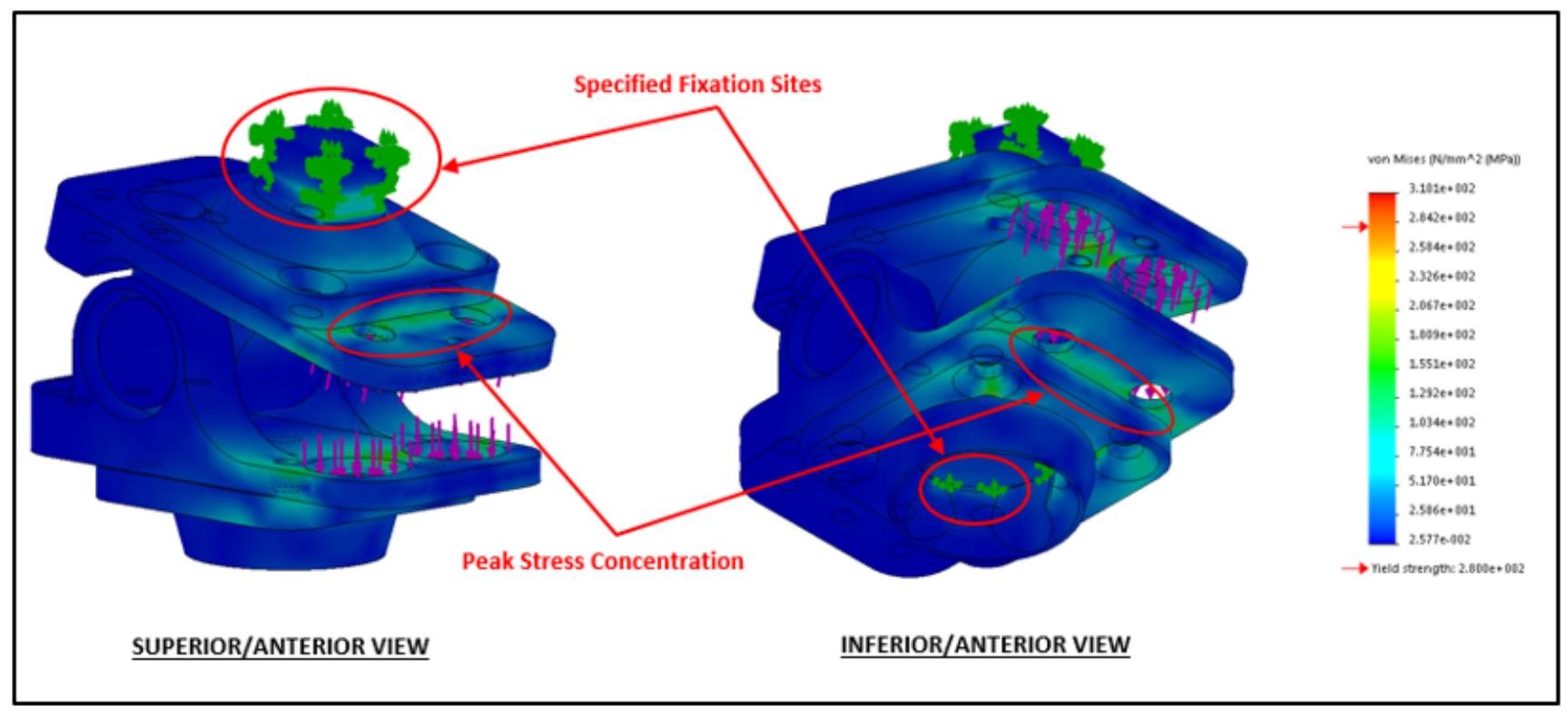

Figure 8.2: Results of FEA Stress Simulation under Peak Dorsiflexion Loading Conditions.

This image displays results of the FEA stres simulation of the AJP under peak dorsiflexion loading conditions. Additionally the image displays the fixation sites assumed in the FEA simulation, as well as the force load distribution at the spring seats. Note that the yield stress of the AJP housing material (Aluminium Alloy 6082-T6) is $280 \mathrm{MPa}$, which is indicated by the red arrow on the colour bar. Thus stresses approaching material yield are indicated by an orange-red colour. The simulation was conducted using SolidWorks Simulation package.

The summarised results of the FEA simulation are displayed in Figure 8.3. In the figure it can be seen that the maximum stress the AJP housing experiences occurs when under peak dorsiflexion of $15^{\circ}$, yet the stress induced is only $\pm 65 \%$ of material yield strength. Thus it is concluded that the design has the structural integrity required to ensure that it can withstand the peak loading during use. 


\section{PEAK DORSIFLEXION}
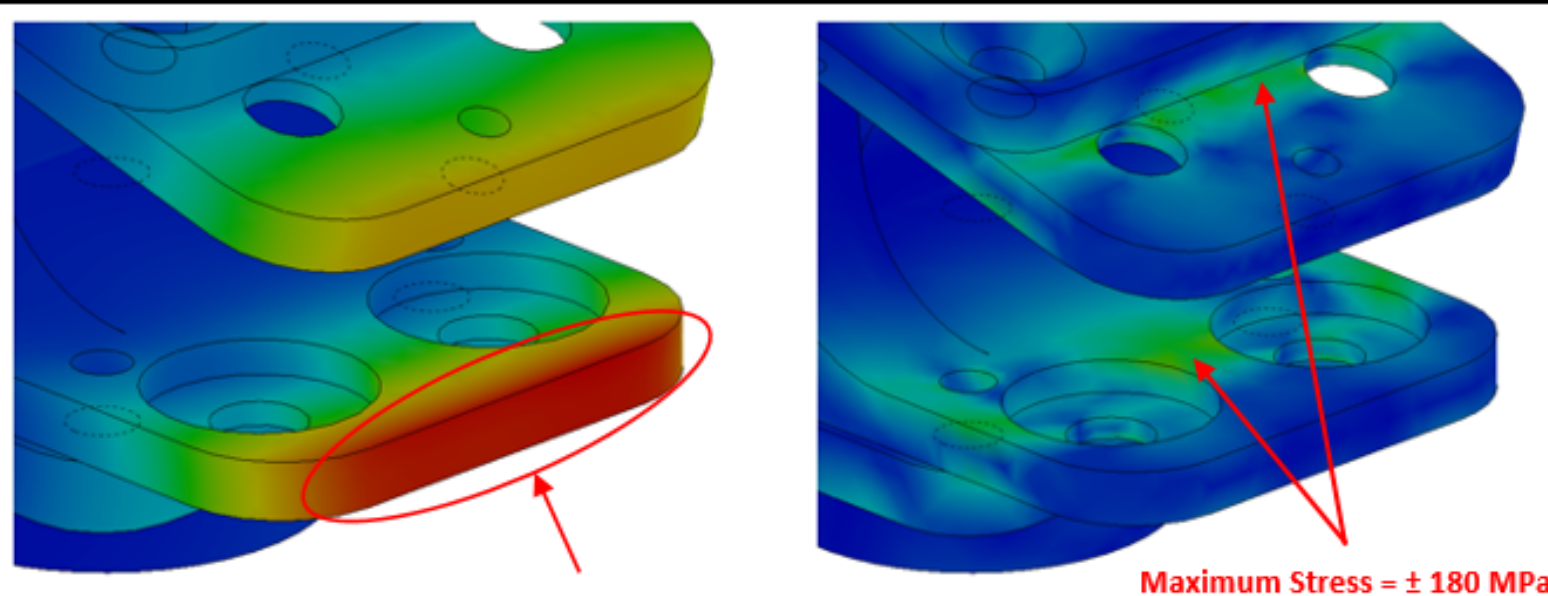

Maximum Deflection $= \pm 0.257 \mathrm{~mm}$

Maximum Stress $= \pm 180 \mathrm{MPa}$

PEAK PLANTARFLEXION

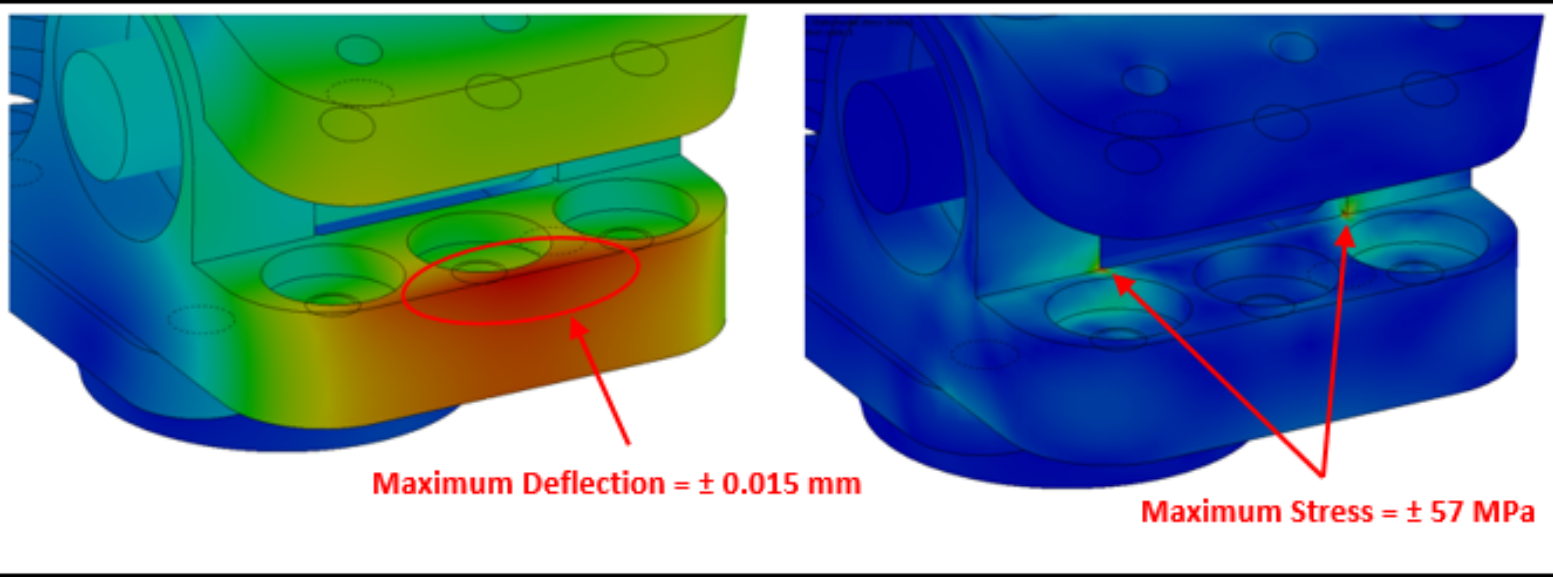

Figure 8.3: Results of FEA Simulation.

This image displays results of the FEA simulation at peak dorsiflexion and plantarflexion load conditions (see Table 8.2). The images on the left display the location and magnitude of maximum deflection under these conditions, whilst the images on the right display the location and magnitude of peak stress. Note that the yield stress of the AJP housing material (Aluminium 6082-T6) is $280 \mathrm{MPa}$. Also note that each image has an independent colour scale. The simulation was conducted using SolidWorks Simulation package. 


\subsection{Axle Calculations}

Figure 8.4 displays the free body diagram (FBD) that is used to determine the peak reaction force on the AJP axle when under peak loading conditions ( $15^{\circ}$ of dorsiflexion).
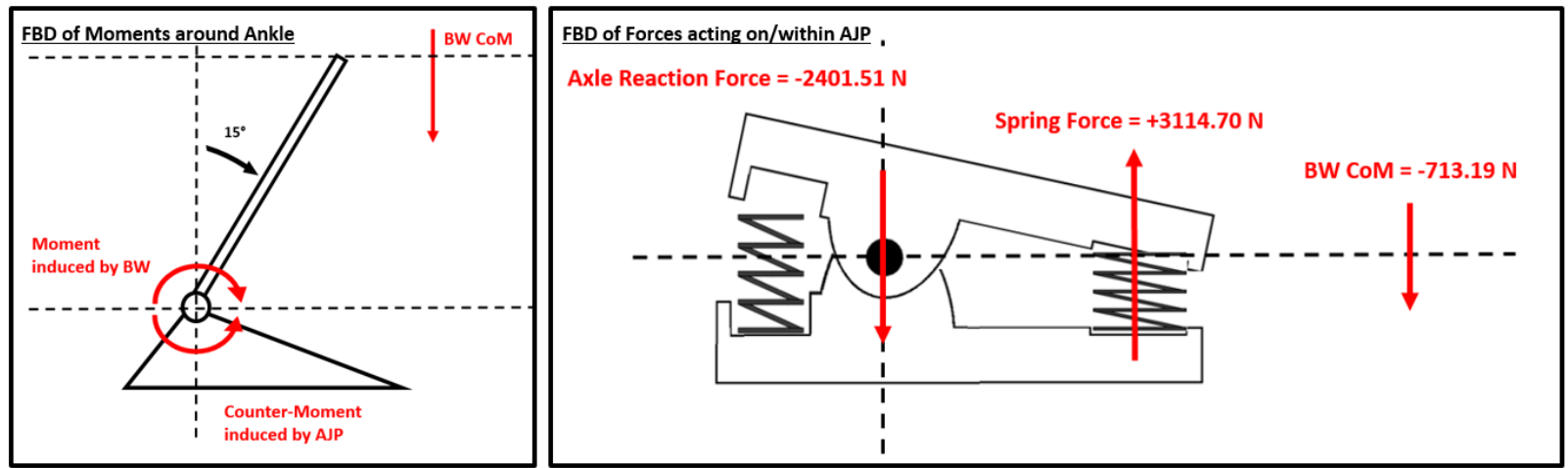

Figure 8.4: Free Body Diagram (FBD) of Forces and Moments acting on AJP.

This image displays a simplified FBD of the forces and moments acting on and around the AJP under peak dorsilfexion load conditions. This FBD is used to determine the reaction force on the AJP axle under these load conditions, with a participant of body weight $72.7 \mathrm{~kg}$ in accordance with the source data [Su et al., 2010]. An assumption made in this analysis is that the forces on or within the AJP are all acting in a vertical LoA.

Thus, the peak force that the axle will incur is approximately $2400 \mathrm{~N}( \pm 3 \mathrm{BW})$. This reaction force is approximated as a load evenly distributed along the length of the portion of the axle that is in contact with the upper portion of the AJP housing, as displayed in Figure 8.5.

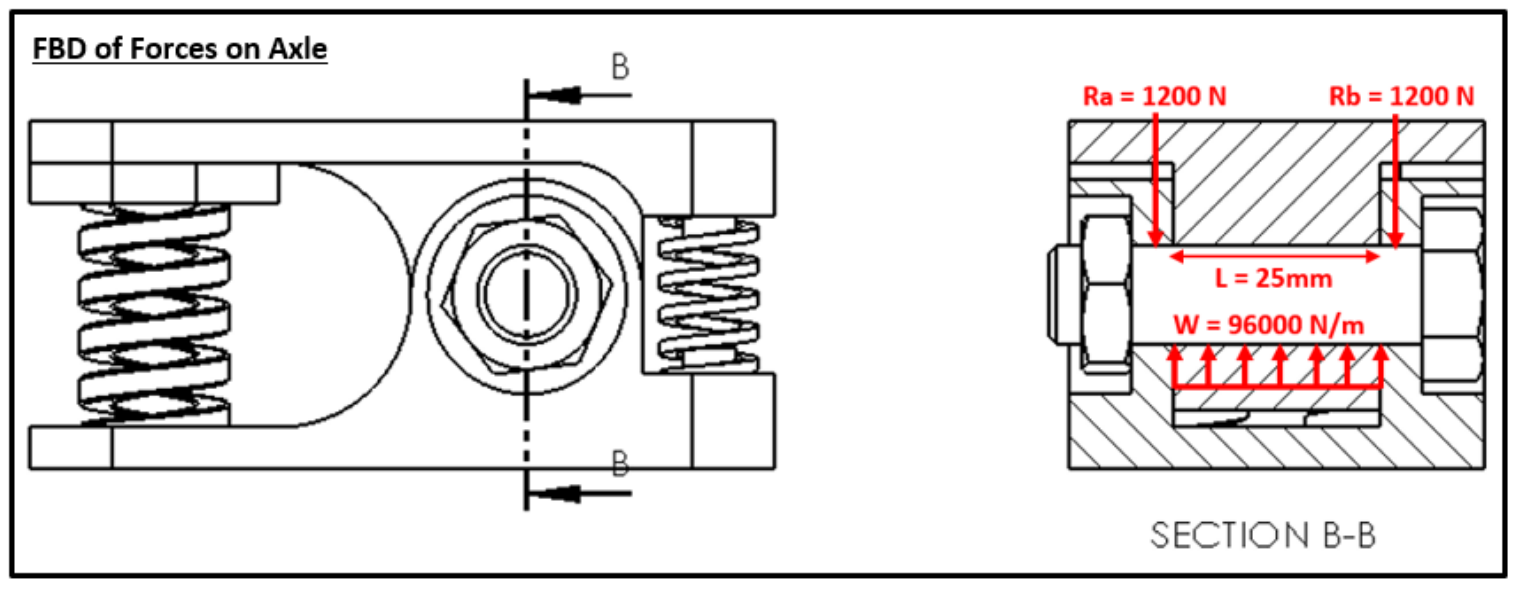

Figure 8.5: Free Body Diagram (FBD) of Forces acting AJP Axle.

This image displays a simplified FBD of the forces acting on the AJP axle during peak loading conditions. Under these conditions an evenly distributed force of $96000 \mathrm{~N} / \mathrm{m}$ is applied onto the portion of the AJP axle that is in contact with the upper support of the AJP housing. The values in the FBD form the inputs for the calculations used to generate the graphs seen in Figure 8.6. 
Calculations according to Budynas and Nisbett [2011] verify that the axle used, a M12 hex head bolt of galvanised steel (modulus of elasticity (E) of $200 \mathrm{GPa}$ and yield strength of $203 \mathrm{MPa}$ ), can withstand this peak stress incurred with a safety factor of 4.59 . The equations used for this analysis are displayed in Appendix B, and the results thereof are displayed in Figure 8.6.
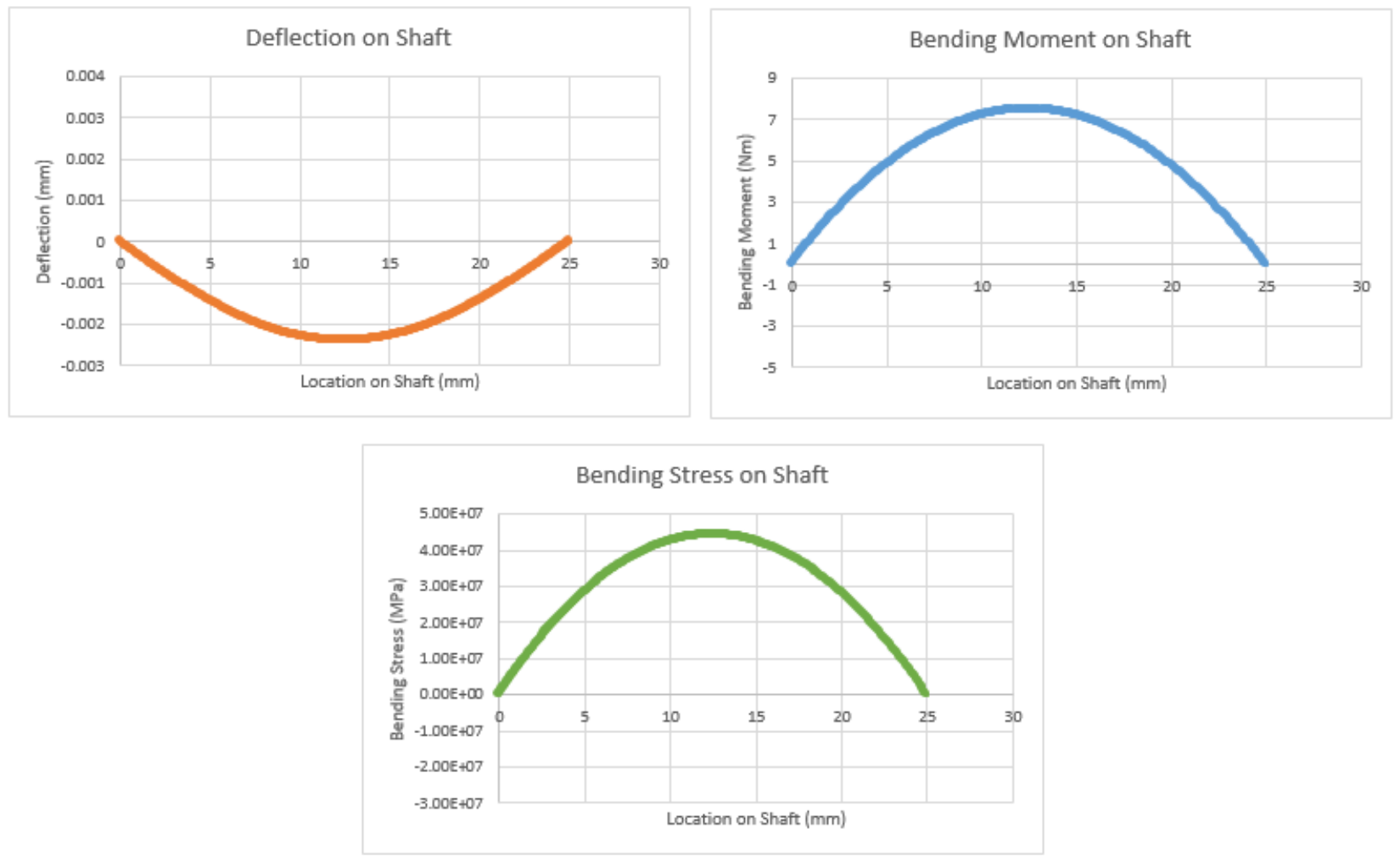

Figure 8.6: Results of Axle Stress and Deflection Calculations.

This image displays results of the calculations done to verify that the AJP axle can withstand the peak loads incurred during peak dorsiflexion load conditions, in accordance with Figure 8.4. The results are as follows: Maximum shaft deflection < 0.0025mm; Maximum shaft bending moment $<8 \mathrm{Nm}$; Maximum shaft bending stress $<50 \mathrm{MPa}$. Galvanised steel has a yield strength of $203 \mathrm{MPa}$, thus the safety factor of the axle is 4.59 . 


\section{Part III}

\section{Experimental Analysis}




\section{Chapter 9}

\section{Experimental Methodology}

\section{Overview}

The AJP designed in this project is based on the quantification of natural stance mechanics detailed in Section 6.2, in an attempt to fulfill the objectives stipulated in Section 6.1.1. The device has been designed to be modular, as it allows for a clinician to insert springs of varying stiffness to accommodate for different amputee weight categories.

The scope of this experimental methodology focuses on the analysis of the mechanical, functional and safety performance of the designed AJP. Due to the ethical implications of involving amputee participants prior to the validation of these metrics, the clinical analysis of the AJP's impact on TT amputee stance mechanics is thus deferred to later research. The AJP will instead be evaluated by a simulation of the stance phase of TT amputee gait cycle via the use of a pseudo-prosthesis (PP) and healthy (able-bodied) participants, a methodology that has been used in literature [Collins and Kuo, 2010], [Hansen et al., 2004]. The results of this study are thus used as indicators of potential success of a future clinical study on TT amputee participants. As the outcomes of the study relate to the mechanical performance of the AJP during this phase, it is deemed acceptable that amputees are not involved in this experimental methodology, and the rationale thereof is discussed using examples in literature in Section 9.1.1. In this way, a PP system is designed, manufactured and assembled. The system, shown in Figure 9.1, consists of three subsystems: (i) an ankle immobiliser boot (AIB), which immobilises the lower leg from mid-tibia to the metatarsal-phalangeal joint of the foot; (ii) the designed AJP, which interfaces to the base of the AIB using a custom designed plate; (iii) a baseline AFP (Otto Bock 1D10), the male pyramid head attachment of which is secured into the female socket of the AJP. AFP's such as the 1D10 are intentionally manufactured inclusive of a heel-to-toe drop of $10 \pm 5 \mathrm{~mm}$, such that they sit appropriately in a shoe. As such it is recommended that they are tested in this intended setup, and thus the PP and DPP apparatus will include an ASICS HN6G2 shoe of heel-to-toe drop of approximately $10 \mathrm{~mm}$.

The aim (see Chapter 5) of this experimental methodology is to evaluate the effect the AJP has on the baseline AFP's sagittal plane stance phase mechanics during simulated TTA gait. As such, 

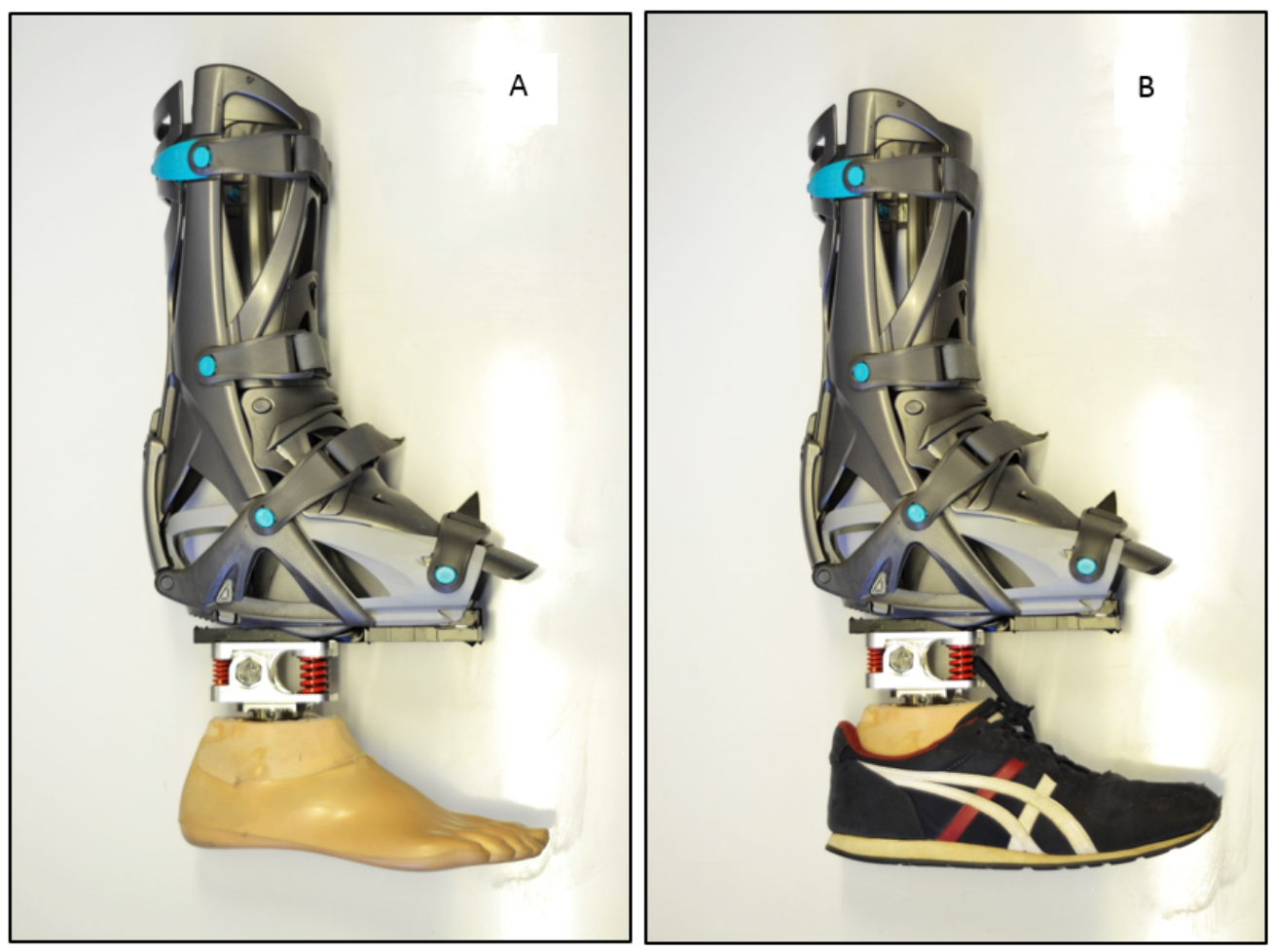

Figure 9.1: The Pseudo-Prosthesis.

This figure displays the final pseudo-prosthesis (PP) with and without the shoe used in this studies experimental methodology. The PP consists of three sub-systems: (i) an ankle immobiliser boot (AIB), which immobilises the lower leg from mid-tibia to the metatarsal-phalangeal joint of the foot; (ii) the designed AJP, which interfaces to the base of the AIB using a custom designed plate; (iii) a baseline AFP (Otto Bock 1D10), the male pyramid head attachment of which is secured into the female socket of the AJP. See Appendix E for detailed drawings of this assembly.

the experimental methodology consists of two core tests, a control test (CON) and an experimental test (EXP), within which the AJP in the PP system is activated and deactivated, respectively. Figure 9.1 displays the activated PP and Figure 9.7 displays the deactivated PP (DPP). Note that the PP system is converted into the DPP system by removing the posterior spring set (the pseudodorsiflexors) and securing a set of solid stainless steel shafts into the spring seats. This swop-out is done between the CON and EXP test, and additionally serves as an indication of the ease of assembly/disassembly of the AJP.

Thus, this chapter describes the experimental methodology, centred around the CON and EXP test. Figure 9.2 displays a flow chart that provides an overview of this methodology. 


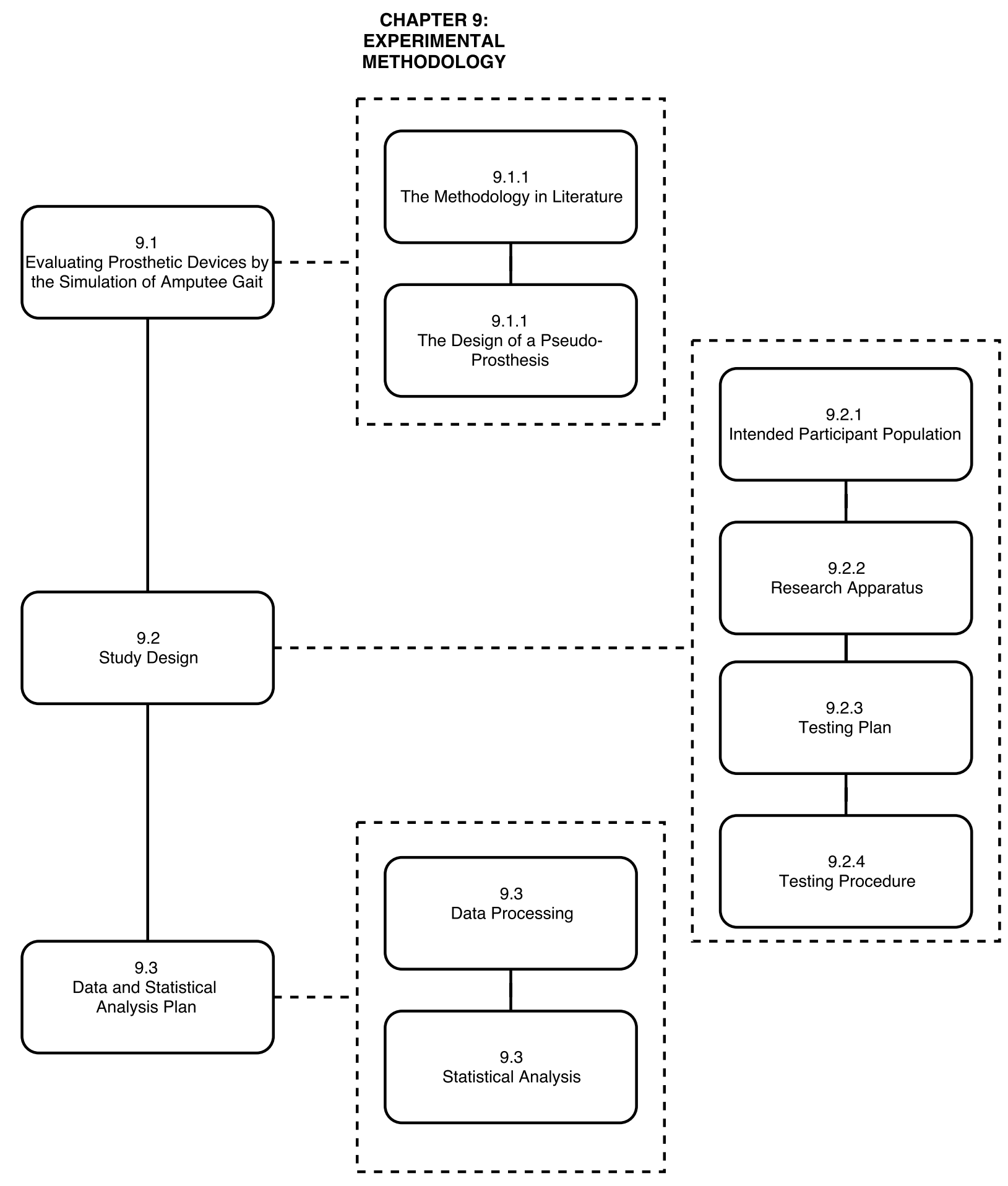

Figure 9.2: Flowchart of the Experimental Methodology.

This figure displays the flowchart that visually describes the experimental methodology designed for this study. 


\subsection{Evaluating Prosthetic Devices by the Simulation of Amputee Gait}

\subsubsection{The Methodology in Literature}

A review of the literature that relates to AFP design has revealed that it is common practice to evaluate the devices using able-bodied subjects and PP. These systems consists of an AIB that have been adapted to allow for the intergration of an AFP sub-systems into its base, as shown in Figure 9.3. These systems thus allow for a simulations of a TT amputation by rendering the subjects' own ankle and associated musculature immobile, translating any angular motion and moment requirements during a stride into the AFP. This will allow for initial validation of the device via an analysis of its mechanics during the simulated stance phase of TT amputee gait using ablebodied subjects. Figure 9.3 displays a visual description of Collins and Kuo [2010] experimental procedure, an example of a study that utilises able-bodied participants to evaluate AFP's using a PP.
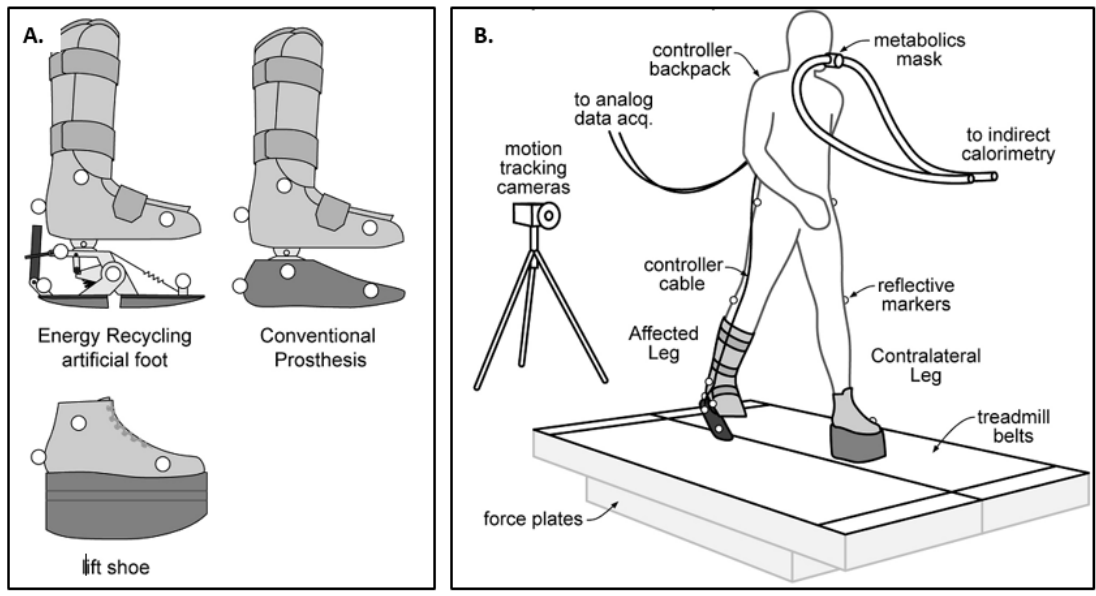

Figure 9.3: CESR Experimental Procedure. [Collins and Kuo, 2010]

This figure provides a visual description of the experimental procedure used by Collins and Kuo [2010], whereby they used a prosthetic simulator ( $A$ ) to examine the influence on gait mechanics of their design (CESR) in comparison to a conventional prosthesis, as well as the associated impact on metabolic energy energy expenditure (B).

The alternative method of AFP evaluation that negates the need for amputee subjects is that of machine bench testing, where the mechanical properties of the AFP's are objectively isolated and examined, and studies that exhaustively validate AFP's prior to amputee interaction adopt testing methodologies that utilise parallel machine and pseudo-gait research protocols. One such study that used this parallel methodology was that of Hansen et al. [2000], for the examination of the relationship between prosthetic alignment and the roll-over shapes of four rigid-ankle AFP's.

Part 1 of their methodology was that of machine-simulation, involving the use of a prosthetic foot loading apparatus (PLFA) seen in Figure 9.4. The PFLA allows for the quasi-static loading of the AFP's at five angular orientations in the sagittal plane, each simulating an orientation that might occur during the stance phase of gait. Combining the results of each angular test allowed for the 
approximation of rollover shape for each AFP tested. Part 2 of the methodology is the simulation of

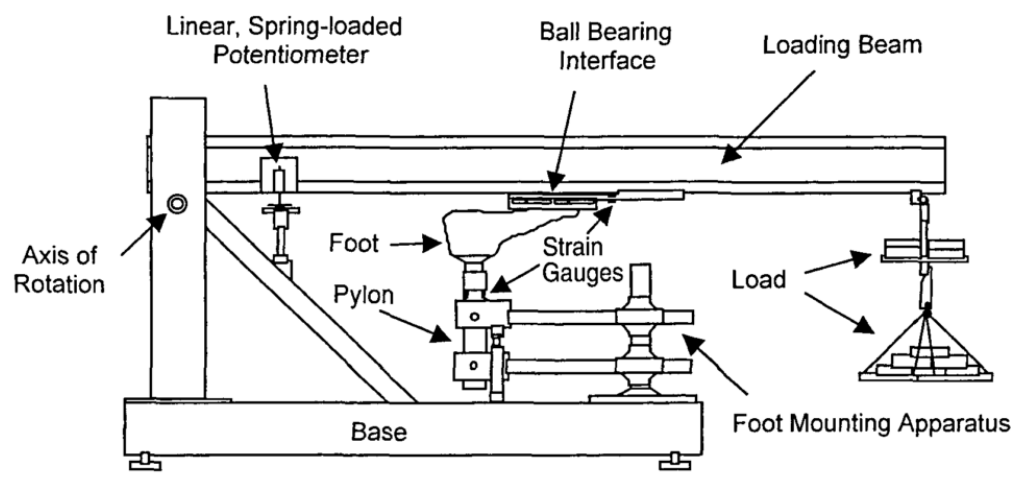

Figure 9.4: The Prosthetic Foot Loading Apparatus. [Hansen et al., 2000]

The PLFA is used for a mechanical quasi-static test of AFP devices. It operates as a second class lever around the Axis of Rotation, with a load applied at a moment arm length longer than that of the AFP reaction force. The Foot Mounting Apparatus allows for adjustments to the angular orientation of the AFP relative to the Loading Beam

the stance phase of gait using able-bodied subjects wearing a PP, which allows for an examination of the roll-over shape using both quasi-static roll-over methods and dynamic roll-over methods. The dynamic method refers to a test that requires the subject to walk normally with the PP. The only variable that changes significantly between these two tests is the speed of loading, as the quasi-static test takes approximately 5 seconds for a complete roll-over whilst the dynamic test takes approximately 0.8 seconds [Hansen et al., 2000]. In comparing the results from each of the three testing methodologies, Hansen et al. [2000] concluded that the AFP's act in a quasi-static manner even when operating during the simulated stance phase of a TTA level walking gait cycle and concluded that they governed by their quasi-static properties.

Whilst a thorough analysis of the AJP designed for this project would ideally involve using the entirety of the methodology used by Hansen et al. [2000], the quasi-static PFLA test requires use of the PFLA itself, or a similar test rig. However, the conclusion of Hansen et al. [2000] provides sound reason to evaluate prosthetic feet using the dynamic roll-over methodology. Therefore the experimental methodology for this project will rely on a dynamic roll-over tests using a PP.

\subsubsection{The Design of Pseudo-Prosthesis}

The PP used for this research was designed and assembled in-house. It consists of three core sub-assemblies: (i) an OPED VACOped (OPED Medical, Inc.; USA), an orthopaedic AIB; (ii) the manufactured AJP; and (iii) a right sided Otto Bock 1D10 foot of length $28 \mathrm{~cm}$. The attachments that interface sub-system 1 and 2 was designed by the research team and manufactured at the UCT Health Sciences workshop (see the drawings thereof in Appendix E). AFP's such as the 1D10 are intentionally manufactured inclusive of a heel-to-toe drop of $10 \pm 5 \mathrm{~mm}$, such that they sit appropriately in a shoe. Thus the PP and DPP apparatus will include an ASICS HN6G2 shoe of 
heel-to-toe drop of approximately $10 \mathrm{~mm}$. Section 7.2.3 describes the interface methodology used to attach the baseline AFP to the AJP. The final assembly of the PP is displayed in Figure 9.1.

The system is designed with the ability to adjust the alignment of the PP in the following ways:

1) The AJP is aligned parallel to the sagittal plane, inferior to the base of the VACOped. There are four possible posterior-anterior positions, each 15mm apart. This will allow for some degree of ankle CoR location shift on site, to ensure accurate replication of a prosthetic tibial-foot alignment.

2) The interface between the AJP and the baseline AFP is analogous to the interface between a pseudo-tibia and baseline AFP. The attachment thus allows for rotational alignment of the baseline AFP relative to the AJP in the transverse, coronal and sagittal plane.

\subsection{Study Design}

\subsubsection{Intended Participant Population}

For the purpose of validating the mechanics of the AJP via the simulation of TT amputee gait cycles, and specifically the stance phase thereof, a subject population consisting of three (3) healthy able-bodied (i.e. non-amputees) volunteers will be sought. For this selection, precision of participant demographic groups is deemed more important than the accuracy of demographic grouping when compared to the ideal study population demographic. As such, participants of a relatively consistent age and mass are to be selected. Ethical clearence for this study has been approved by the UCT's Human Research and Ethics Committee (HREC), reference number 542/2017 (see Appendix F).

\subsubsection{Research Apparatus}

The experimental procedure for this study sets out to analyse the mechanics (kinematics and kinetics) of the AJP during a pseudo-stance phase that intends to replicate the stance phase mechanics of the natural gait cycle. The specific kinetic and kinematics variables to be observed are foot rotation around the ankle during CPF and CDF, and the moment profile that is associated with this motion. From these variables, the ankle stiffness profile (moment vs angular rotation) during this pseudo-stance can be assessed. Thus the relationship between angular rotation and moment in the sagittal plane needs to be described, which will be done by using a motion capture camera system and force feedback apparatus.

Such a set-up can be found at UCT's Biomechanics Laboratory, located at the Sports Science Institute of South Africa. The facility has an 8-camera Vicon motion capture system (Oxford Metrics, Oxford, UK), a photogrammetry apparatus that is used to track marker motion in three dimensional space. This system will be used to track markers placed on the PP at $250 \mathrm{~Hz}$. Further, the facility 


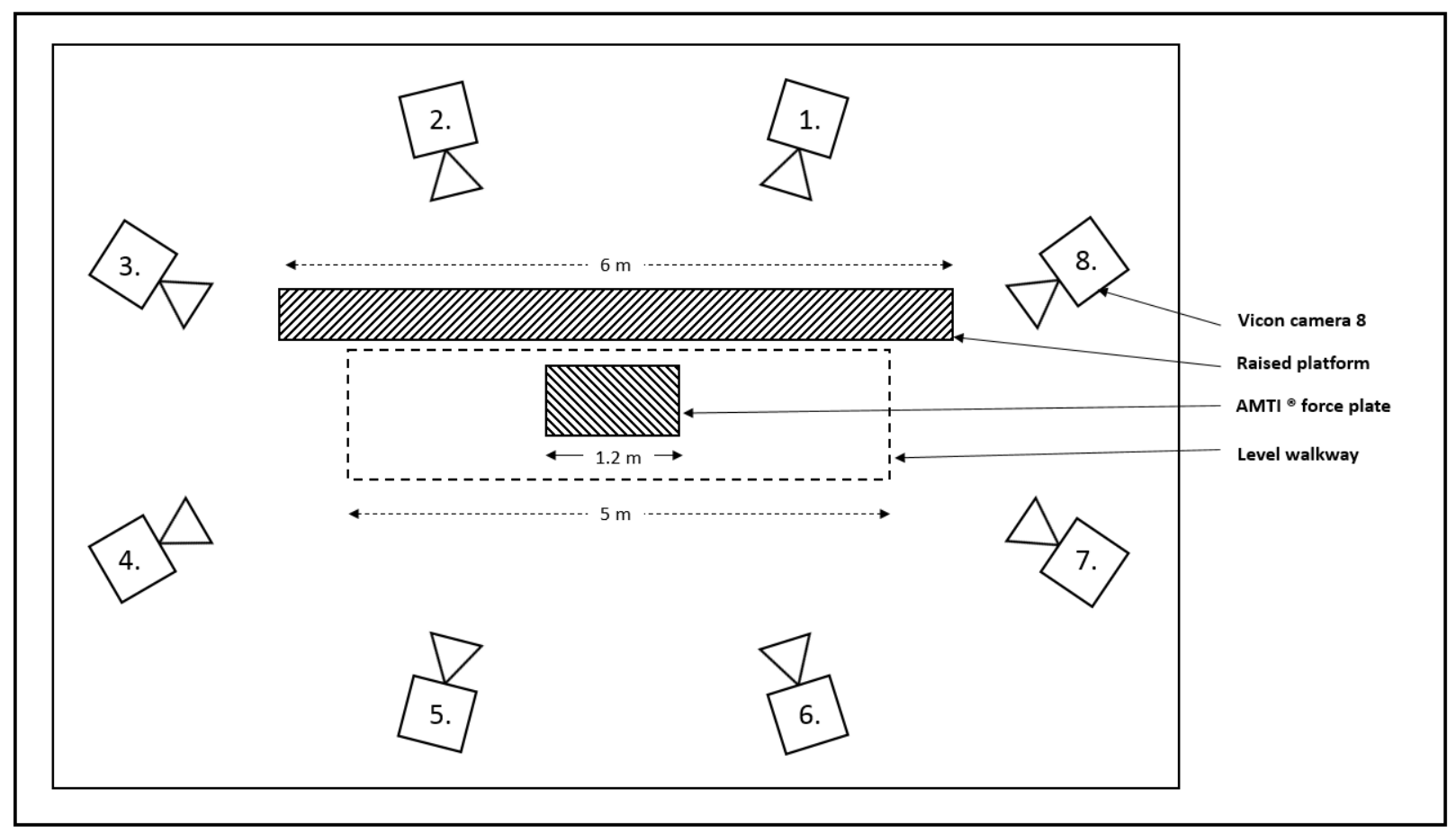

Figure 9.5: Kinetic and Kinematic Measurement Apparatus

This figure displays a birds eye view of the testing apparatus to be used, located at UCT's Biomechanics Laboratory at SSISA. The system consists of an 8-camera Vicon motion capture apparatus (Oxford Metrics, Oxford, UK) and one $1200 \mathrm{~mm} \times 600 \mathrm{~mm}$ AMTI (R) FP (AMTI, Watertown, MA, USA). The Vicon system directs its focus towards the $5 \mathrm{~m}$ straight level walkway, at the centre of which the FP is embedded. A raised platform, of height equal to the leg length extension associated with the PP/DPP, is aligned parallel to the walkway.

has a AMTI (B) FP apparatus (AMTI, Watertown, MA, USA) that allows for the capture the magnitude and centre of pressure (CoP) of ground reaction force (GRF) vectors. This plate will record GRF vectors at $1000 \mathrm{~Hz}$, the CoP of which will be synced with the Vicon system and thus allows for the quantification of stance mechanics. The marker system used to describe gait kinematics is detailed in Section 9.3.1. This is based on an adapted Helen Hayes [Kadaba et al., 1990] model [Hansen et al., 2004] and is displayed in Figure 9.9.

Hansen et al. [2000] and Collins and Kuo [2010] both compensated for the leg length increase by attaching a PP to both the left and right leg of the subject, the focus limb utilising an assembly that consisted of the focus device and the contralateral limb utilising an assembly that consisted of a control device, and examined both limbs as part of their experimental procedure. Yet, the force capture apparatus available for this project limits data analysis to one stride per test. Thus to balance the participants stride, a raised platform of height equal to the extension length is aligned parallel to the walkway. Figure 9.6 presents a description the research location apparatus. 

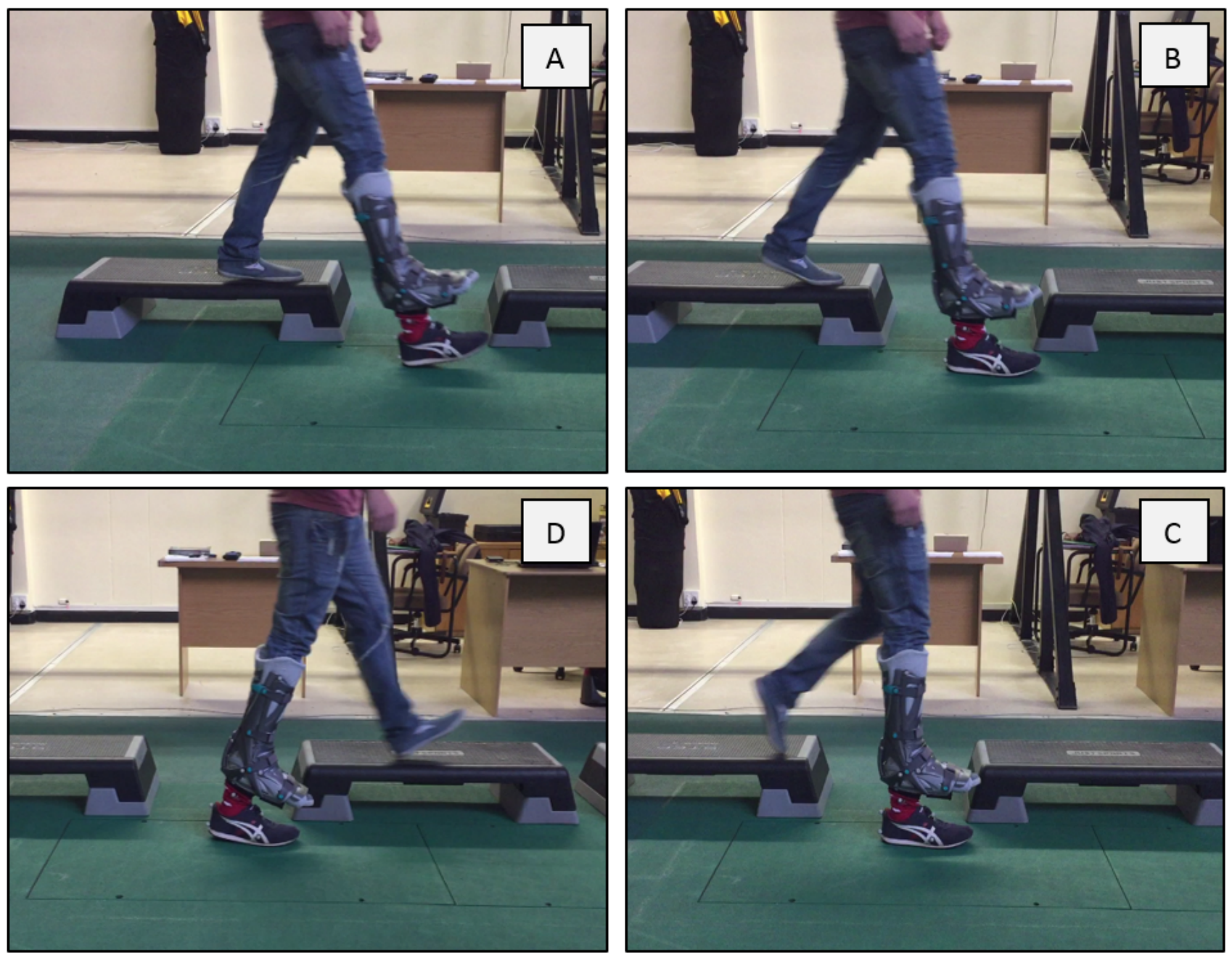

Figure 9.6: The Simulation of Trans-Tibial Amputee Gait

This figure displays the methodology used to simulate TT amputee gait via the use of the PP, an able-bodied participant and the research apparatus. Clockwise from top left is displayed examples of: heelstrike; footflat; mid-stance; and peak dorsiflexion as the heel begins to rise. Also observe in the figure that the FP is embedded into the ground, the participant walks with their right limb in the PP and their left limb balancing the height difference on a raised platform, and the reflective markers placed on the $P P$.

\subsubsection{Testing Plan}

The experimental procedure is divided into two core tests, a control test (CON) and an experiment (EXP). The EXP test utilised the PP as designed and described in Section 9.1.2, and derives the major outcomes of this testing plan. Yet to quantify the effect of the AJP based on the data derived from the EXP, the PP will be adjusted in the CON test such that the AJP is deactivated using solid stainless steel shafts that thread into the spring seats wherein the springs would normally sit, thus locking the AJP. This adjustment limits all mobility within the AJP, and as such all angular rotation and moment response during a gait cycle using this deactivated PP (DPP) is ideally localised to 
the baseline AFP itself. Thus the CON test will allow for stance mechanics quantification of the baseline AFP, whilst the EXP test will generate that of the baseline AFP + AJP. See Figure 9.7 for a visual representation of these changes, and Appendix $E$ for assembly drawings of the DPP.

It cannot, however, be assumed that the CON test will localise all stance phase mechanics to the baseline AFP, as it is likely that the PP/DPP structure (specifically the AIB and the shoe) will have inherent margins of flexibility that is difficult to estimate. This creates an unquantified margin of error. Yet in this methodology the margin of error is eliminated with the assumption that the inherent flexibility of the DPP is the same of that in the PP. Thus by comparing the stance mechanics of the PP in the EXP test to the stance mechanics of the DPP in the CON test, an indication of the improved mechanics introduced by the AJP can still be derived. This is the principle goal of the experimental procedure.
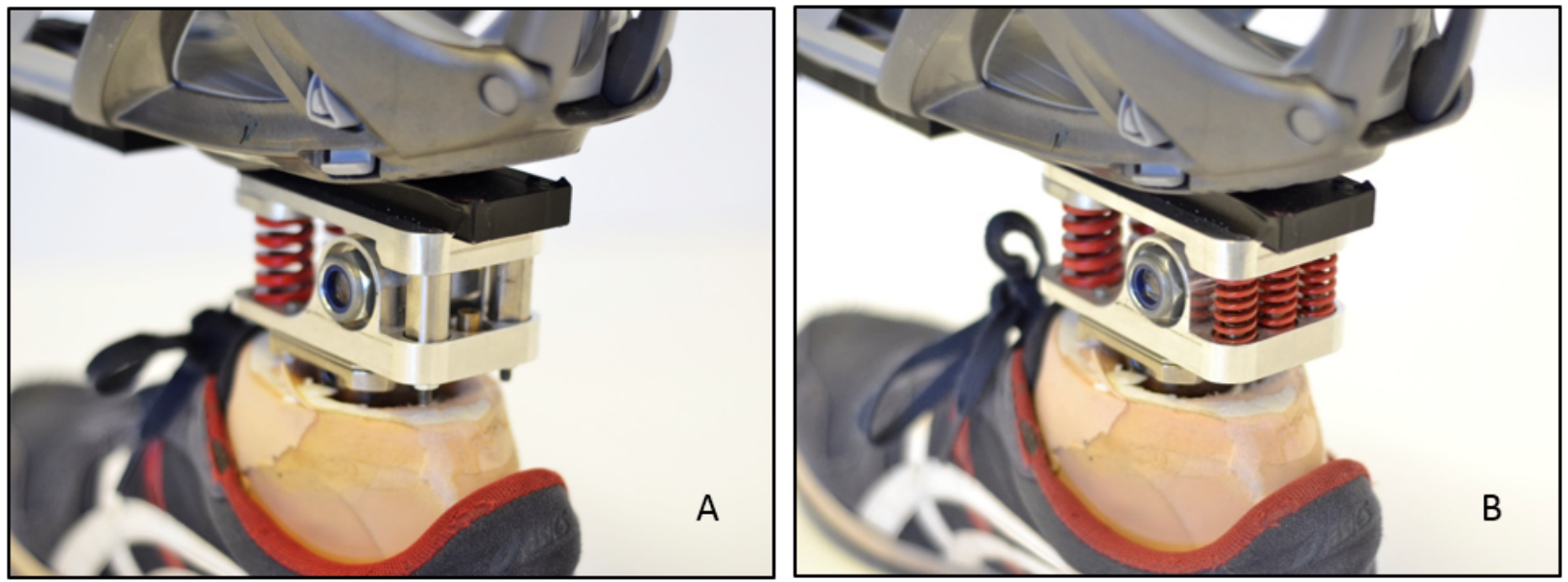

Figure 9.7: The Deactivated Pseudo-Prosthesis (DPP) and the Pseudo-Prosthesis (DPP).

This figure displays a close up of both the $D P P(A)$ and $P P(B)$ used in this experimental methodology. The $P P$ is converted into the DPP by the removal of the AJP's posterior spring set (i.e. the pseudo-dorsiflexors) and securing a set of solid stainless steel shafts into the spring seats. This immobilises the AJP in both plantarflexion and dorsiflexion, and thus transfers all stance mechanics into the baseline AFP. See Appendix E for detailed drawings of this adjustment.

\subsubsection{Testing Procedure}

The testing procedure that follows is visually described in Figure 9.8. 


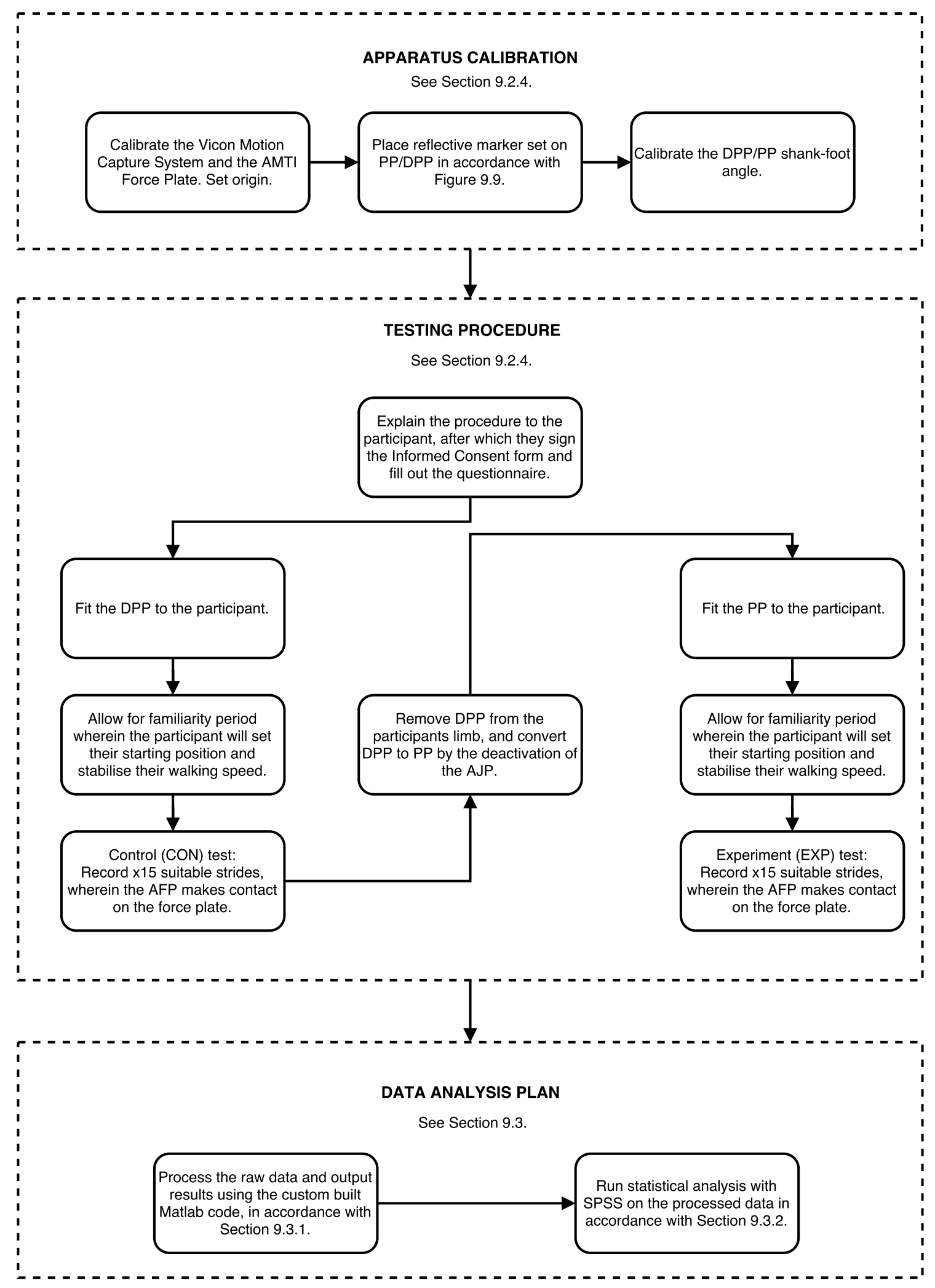

Figure 9.8: Flowchart of the Testing Procedure and Data Analysis Plan.

This figure displays the flowchart that visually describes the apparatus calibration, the testing procedure and an overview the data analysis plan designed for this study. Note that this procedure is repeated for each participant. 
The research team will arrive at the location prior to the arrival of the participant, and begin the apparatus set-up. This will primarily consist of system calibration, namely:

\section{The Calibration of the Vicon and AMTI FP}

The 8-camera Vicon system is calibrated using the standard Vicon calibration procedure. Subsequently, the origin of the system is defined to the corner of the AMTI FP closest to camera 3. The FP is also zero'd.

\section{The Placement of the Marker Set on the PP/DPP.}

The custom 7 point marker set will be placed on the PP/DPP, in a manner approximated as shown in Figure 9.9. This set-up is an adaptation of the marker set used by [Hansen et al., 2004], which itself is an adapted Helen Hayes marker set [Kadaba et al., 1990]. This data processing methodology is used to calculate kinetics of stance (described in Section 9.3.1). This marker set-up attempts to create a perpendicular shank-foot angle.

\section{Calibration of PP/DPP Shank-Foot Angle.}

As the marker set will be hand placed on the DPP/PP by a trained member of the research team (in accordance with Section 9.3.1), the shank-foot angle created by the markers will have a margin of error that needs to be accounted for by calibration within the Vicon system. Thus the assembled PP/DPP + marker set will be placed onto the walkway, aligned with the sagittal plane, and the shank-foot angle will be measured. This will allow the research team to computationally set the neutral shank-foot angle to zero in post-processing, thus allowing any rotation around the AJP ankle joint to be indicative of plantar- and dorsi-flexion. Thus the angle preceding heelstrike is expected to be zero. By calibrating this angle before each participant interaction with the PP/DPP, any margin of error associated with the placement of the markers will be eliminated.

Upon the arrival of the participants, each one will individually be processed in accordance with a procedure approved by the HREC, which involves giving consent and the completion of a questionnaire that ensures they are eligible for the study. The weight and height of the participants is measured on site. The PP/DPP is then fitted to the right lower leg of the participant, who will then be assisted to the walkway. The walkway is a five meter level (flat) pad that consists of one AMTI (R) FP, and the raised level for the contralateral limb (left) is set parallel to the walkway (refer to Figure 9.6). The participant will be encouraged to acclimatise to the PP/DPP. The following tests will then be carried out: 


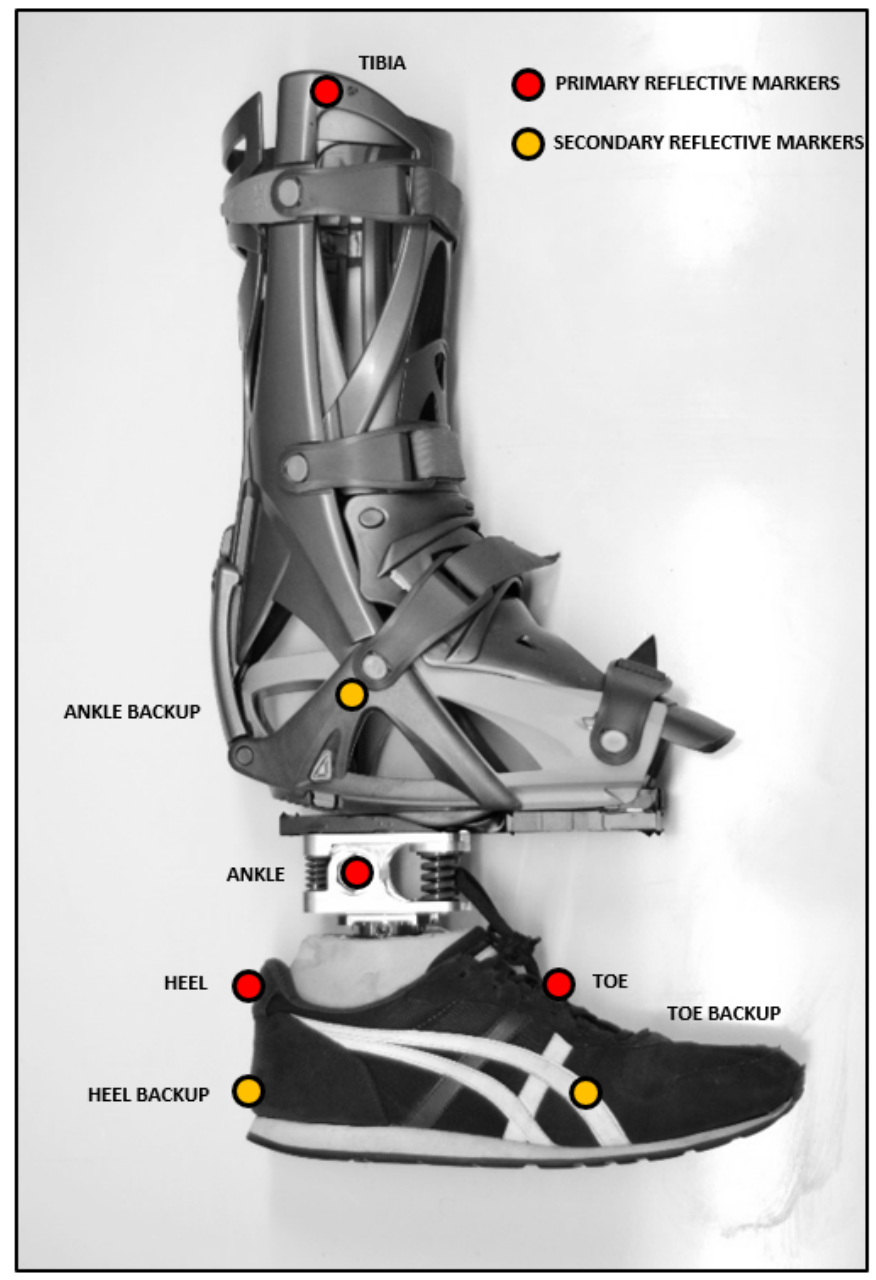

Figure 9.9: The Motion Capture Marker System.

This figure displays the reflective markers placed on the PP/DPP for kinematic motion capture using the Vicon system. Red markers are the primary markers, and allow for the approximation of a tibial-ankle line that rotates relative to the heel-toe line. These two lines are assumed to be rigid bodies, and thus the relative motion is directly related to the relative rotation of the inferior and superior AJP plates around the pivot. The yellow markers are secondary markers for the purpose of assuring complete motion capture should any of the primary markers be hidden from camera focus during testing. Further, the secondary heel-toe line is used to prove the rigid body assumption of the foot is a valid assumption, via the analysis of its angular change in comparison to that of the primary heel-toe line.

\section{The Control Test (CON).}

This test will consist of $x 15$ suitably recorded trials (i.e. strides), and will utilise the DPP apparatus as described in Section 9. Figure 9.7 displays the locking adjustment made to the AJP. The participants will be asked to walk along the level walkway, beginning from a standing start and initiating the walk with the contra lateral limb (left). Following the footflat of the contralateral stride, the immobilised leg will initiate its swing phase and proceed to heel strike of the baseline AFP foot. For each trial the participants will be asked to ensure that the PP/DPP heelstrike and subsequent rollover is placed on the AMTI FP. Note that the FP is 
only sized $(1200 \mathrm{~mm} \times 600 \mathrm{~mm})$ to collect force information on a single stride, and therefore only one stride test at a time can be recorded. Thus following the rollover the participants will be allowed to continue walking for a few steps, to ensure that they do not abruptly end their gait pattern, but will then return to the starting position. The motion markers attached to the pseudo-prosthesis will assess the kinematics of the entire cycle by the Vicon system.

The participants will be asked to walk at a comfortable free velocity that they feel is normal, and are encouraged to walk at this velocity across all tests. Thus the starting position (or 'run up') will be dictated by a distance from the FP that the participants feel is appropriate given the instruction to ensure PP/DPP stance occurs on the FP. As such the participants will be given numerous attempts to finalise this 'run-up' length and 'free walking velocity'. Once the participants indicate that they have finalised these variables, the actual testing will commence. The research team will begin recording x15 strides. If the research team notices that one stride failed in terms of amputee balance control, or in terms of equipment failure, that stride test will be noted and an extra stride test will be recorded.

\section{The Experimental Test (EXP).}

This test will consist of the same control methodology as described in the CON test, with the exception that the DPP will be converted into the PP (see Figure 9.7).

\subsection{Data Analysis Plan}

\subsubsection{Data Processing}

The marker set joint kinematics (recorded by the 8-camera Vicon system at $250 \mathrm{~Hz}$ ) and the GRF vector data (recorded by the AMTI $(R) F P$ at $1000 \mathrm{~Hz}$ ) for each test will be analysed using a custom-built MATLAB (R) (Mathworks, Natick, USA) code. The GRF magnitude and COP are filtered using a 4th order zero-lag ${ }^{1}$ low-pass butterworth filter ${ }^{2}$ at $100 \mathrm{~Hz}$, whilst the joint angle datasets are smoothed using rolling averages method (10 points).

Shank-foot angle (i.e. plantar- and dorsi-flexion) is zero'd at a perpendicular angle, adjusted for the calibration angle of the marker set, as displayed in Equation 9.1. Thus the neutral ankle angle (thus the angle just prior to heelstrike) is expected to be $0^{\circ}$. Indication of heelstrike initiation will be taken at when GRF $>=15 \mathrm{~N}$. The GRF vector is presented in two directions, $\mathrm{X}$ (direction of travel in the sagittal plane $=$ positive) and $Z$ (upwards = positive). One period of stance will run from heelstrike to toe-off of the focus limb. This period will be presented as a percentage of a completed stance phase (0\% to $100 \%)$.

$$
\text { Ankle Angle }()=90^{\circ} \text { - (Shank Angle - Foot Angle) - Calibration Angle }
$$

\footnotetext{
${ }^{1}$ Otherwise referred to as forward-backward filtering, where the data is filtered in each direction. This ensures no phase delay of true datapoints.

${ }^{2} \mathrm{~A}$ low-pass butterworth filter is a passband filter that blocks datapoints above a certain cutoff frequency.
} 
Joint moments are typically calculated using Vicon's Plug-In Gait interface and inverse dynamics. However, the programme is built with a modified Helen Hayes marker set that assumes variables associated with typical human anthropometrics and mass distributions. Thus the lower leg length extension renders these assumptions inaccurate for this study, and creates a complication when attempting to calculate joint moments. An approach that negates the need to use inverse dynamics is thus used, based on the methodology used by Hansen et al. [2004] wherein ankle joint moments of human participants were calculated with the assumptions of static equilibrium over the course of the stance phase and a static weightless link between COP and the CoR (in this case the AJP's CoR). This methodology was found to produce acceptably similar results for ankle joint moment calculations in comparison to the inverse dynamics approach [Wells, 1981] at slow to normal walking speeds. For the use of this methodology in this study, Figure 9.10 displays a visual aid for Equation 9.2. Further, the ankle moment will be normalised by body weight (BW).

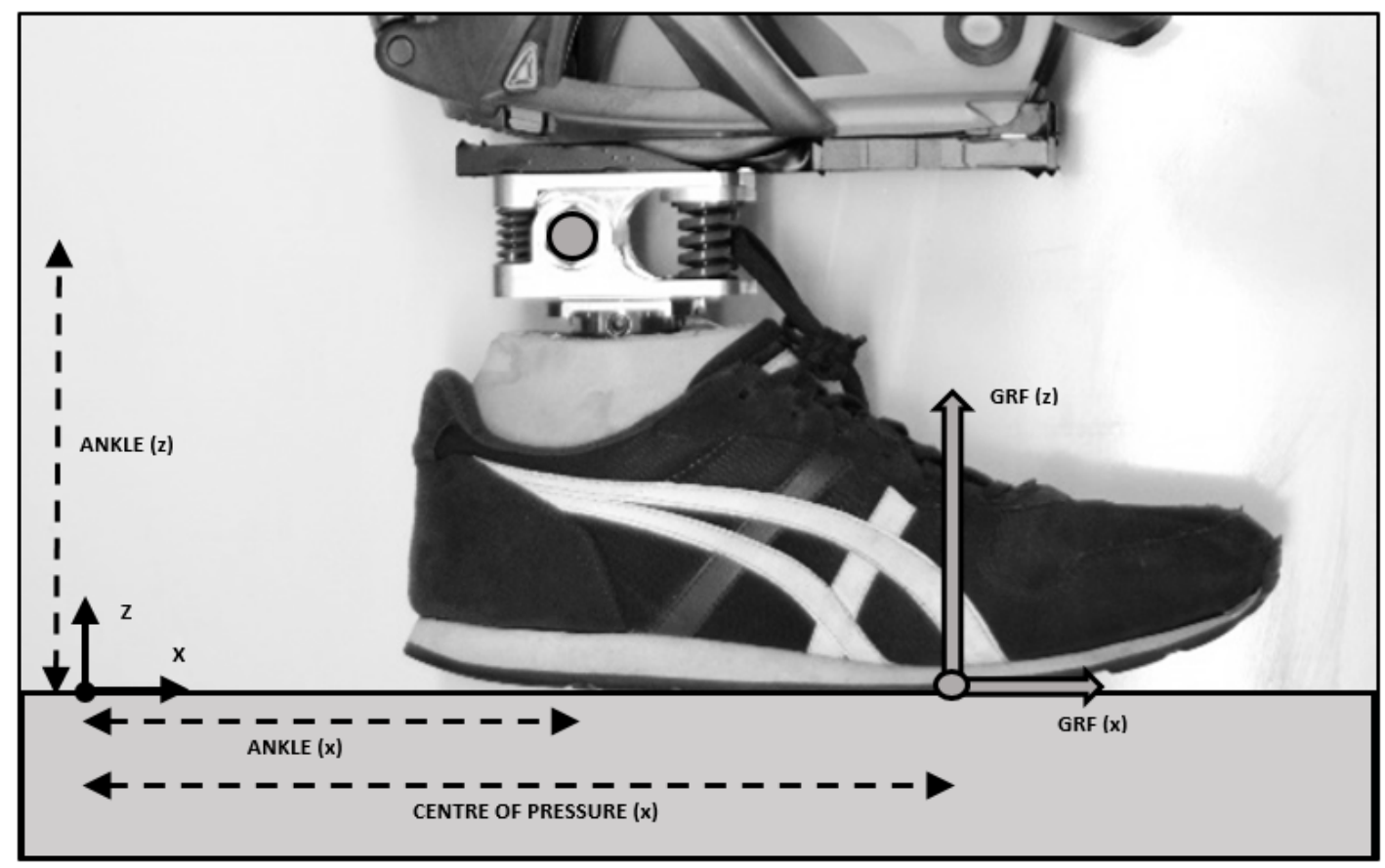

Figure 9.10: Visual Representation of Ankle Moment Calculation.

This figure displays the methodology used for the calculation of ankle moment during stance, and is to be understood in conjunction with Equation 9.2. This methodology is an adaptation of Hansen et al. [2004]. All distances are measured relative to the FP origin. 


$$
\begin{array}{r}
\text { if } \operatorname{CoP}(x)>\operatorname{Ankle}(x) \\
\text { Ankle Moment }=(\operatorname{GRF}(z) \times(\operatorname{CoP}(x)-\operatorname{Ankle}(x)))+(\operatorname{GRF}(x) \times \operatorname{Ankle}(z)) \\
\text { if } \operatorname{CoP}(x)<\operatorname{Ankle}(x) \\
\text { Ankle Moment }=-(\operatorname{GRF}(z) \times(\operatorname{Ankle}(x)-\operatorname{CoP}(x)))+(\operatorname{GRF}(x) \times \operatorname{Ankle}(z))
\end{array}
$$

where postive $G R F(x)$ is in the direction of travel, positive $G R F(z)$ is directed upwards, and positive moment is anticlockwise

Ankle stiffness during CPF and CDF phases of stance are estimated using the assumption of a linear relationship between moment and angular displacement during those respective phases, and is estimated as displayed in Equation 9.3. Note that 'CPF/CDF Peak moment' refers to the value that corresponds to the 'Peak Angle', and is thus not necessarily the absolute peak moment.

CPF Phase Stiffness (Nm/rad.kg) = CPF Peak moment $(\mathrm{Nm} / \mathrm{kg}) \div$ CPF Peak Angle (rad)

CDF Phase Stiffness $(\mathrm{Nm} / \mathrm{rad} . \mathrm{kg})=$ CDF Peak moment $(\mathrm{Nm} / \mathrm{kg}) \div \mathrm{CDF}$ Peak Angle (rad)

\subsubsection{Statistical Analysis}

Intra-participant statistical analysis will be conducted on each participants datasets using SPSS Statistics (IBM, Chicago, USA). An example of this methodology is displayed in Appendix I.

In summary, there are 10 datasets that are to be analysed for each CON and EXP tests of each participant: $x 1$ temporal-spatial (TS) group of $x 4$ variables and $x 1$ kinetic and kinematic (KK) group of $x 6$ variables. The $x 4$ TS variables are stride length $(\mathrm{m})$, walking velocity $(\mathrm{m} / \mathrm{s})$, and the point at which peak angle of CPF and CDF occur as a percentage of the stance phase. The $\times 6 \mathrm{KK}$ variables are peak ankle moment, peak ankle angle, and ankle stiffness at peak ankle angle, for CDF and CPF.

The quartiles ranges of each dataset will be calculated, with which datapoints that are a factor of $x 3$ greater than the interquartile range ${ }^{3}$ are to be designated as outliers. If a datapoint is designated as an outlier, the entire trial will be removed from both the KK and the TS datasets. Once outliers are removed, each dataset will be assessed for normality of distribution using the ShapiroWilk Test for Normality (S-W) [Shapiro and Wilk, 1965], a null hypothesis of a normally distributed dataset, and an alpha value of $\alpha=0.05^{4}$. The results from each S-W test will be reported. Addi-

\footnotetext{
${ }^{3}$ The interquartile range is a measure of statistical dispersion, otherwise referred to as the middle $50 \%$ of the data spread i.e. the 75th percentile - 25th percentile

${ }^{4}$ If the $\mathrm{p}$ value is less than the $\alpha$ value, then we can reject the null hypothesis, thus providing evidence that the dataset is not normally distributed
} 
tionally, SPSS will be used to generate the mean value of the dataset and $95 \%$ confidence intervals $(95 \% \mathrm{Cl})$.

Intrasubject analysis will be conducted on the difference in means between the CON and EXP values of each analysed variable, using an independent t-test, a null hypothesis that the difference in means is equal to zero, and an alpha value of $\alpha=0.05$. A Levene's Test for equality of variance [Levene, 1960] will determine whether the assumption of equal variance can be used.

Due to the small participant sample size of this study, inter-subject statistical analysis will not be conducted, but relevant trends will be observed. 


\section{Chapter 10}

\section{Experimental Results}

This chapter details the results of the experimental methodology described in Chapter 9. Section 10.1 details the graphical and tabulated results of each of the three participants, and contains additional information relevant to the testing methodology of that participant. A summary of the demographics of each participant is displayed in Table 10.1, where they are identified by their initials and a participant number that will from this point forward be their individual referral.

Table 10.1: Participant Demographics.

\begin{tabular}{l|l|l|r} 
& \multicolumn{2}{|l|}{$\begin{array}{l}\text { Participant 1: } \\
\text { 'ACD' }\end{array}$} & $\begin{array}{l}\text { Participant 2: } \\
\text { 'SMT' }\end{array}$ \\
\hline Sex & Male & Male & 'SPB' \\
\hline Age (yrs.) & 21 & 24 & 29 \\
\hline Mass (kg) & 81.5 & 85.0 & 85.0 \\
\hline Height (m) & 1.93 & 1.83 & 1.80 \\
\hline
\end{tabular}

\subsection{Intra-Participant Results and Analysis}

This section details the tabulated results of each participant, in tabulated and graphical form.

Tables 10.2 , 10.4 and 10.6 details the mean of the temporal spatial datasets and a $95 \% \mathrm{Cl}$ for both the CON and EXP tests; the results of the Shapiro-Wilk normality test for each variable; the impact of the AJP as a percent increase/decrease of the mean CON results; and the results of the independent T-test when comparing the difference in CON and EXP means. Tables 10.3, 10.5 and 10.7 detail the mean of kinetic and kinematic datasets and a $95 \% \mathrm{Cl}$ for both the CON and EXP tests; the results of the Shapiro-Wilk normality test for each variable; the impact of the AJP as a percent increase/decrease of the mean CON results; and the results of the independent T-test when comparing the difference in CON and EXP means. Figures 10.1 and 10.2 serve as a graphical representation of the ankle angle and torque profiles against a full stance period. These graphs provide an opportunity to visualise the impact that the AJP has on the baseline AFP stance mechanics (EXP vs CON). The data is taken from the final results of one of the participants 
(Participant 2). Please refer to the Appendix J, where these profiles for Participant 1 and 3 are displayed.
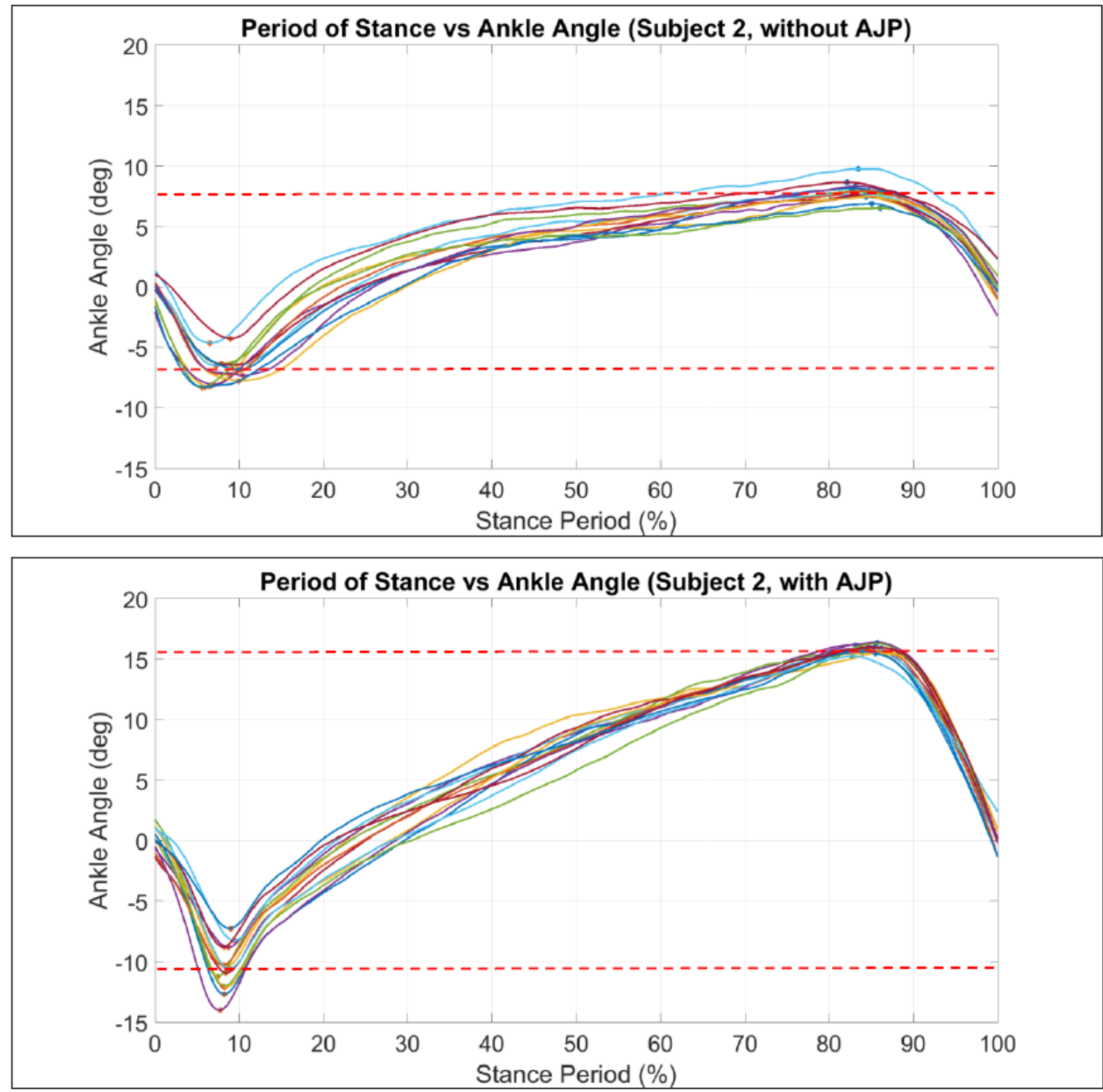

Figure 10.1: Period of Stance vs Period of Stance of Participant 2.

This figure displays the profile of ankle angle against the period of stance of the CON tests (top) and EXP tests (bottom) of Participant 2. The horizontal dashed red lines indicate the mean peak ankle angles during CPF and CDF for each dataset. Note that due to the removal of outliers identified during the statistical analysis of the data, both the CON and the EXP tests only contain 13 samples. 

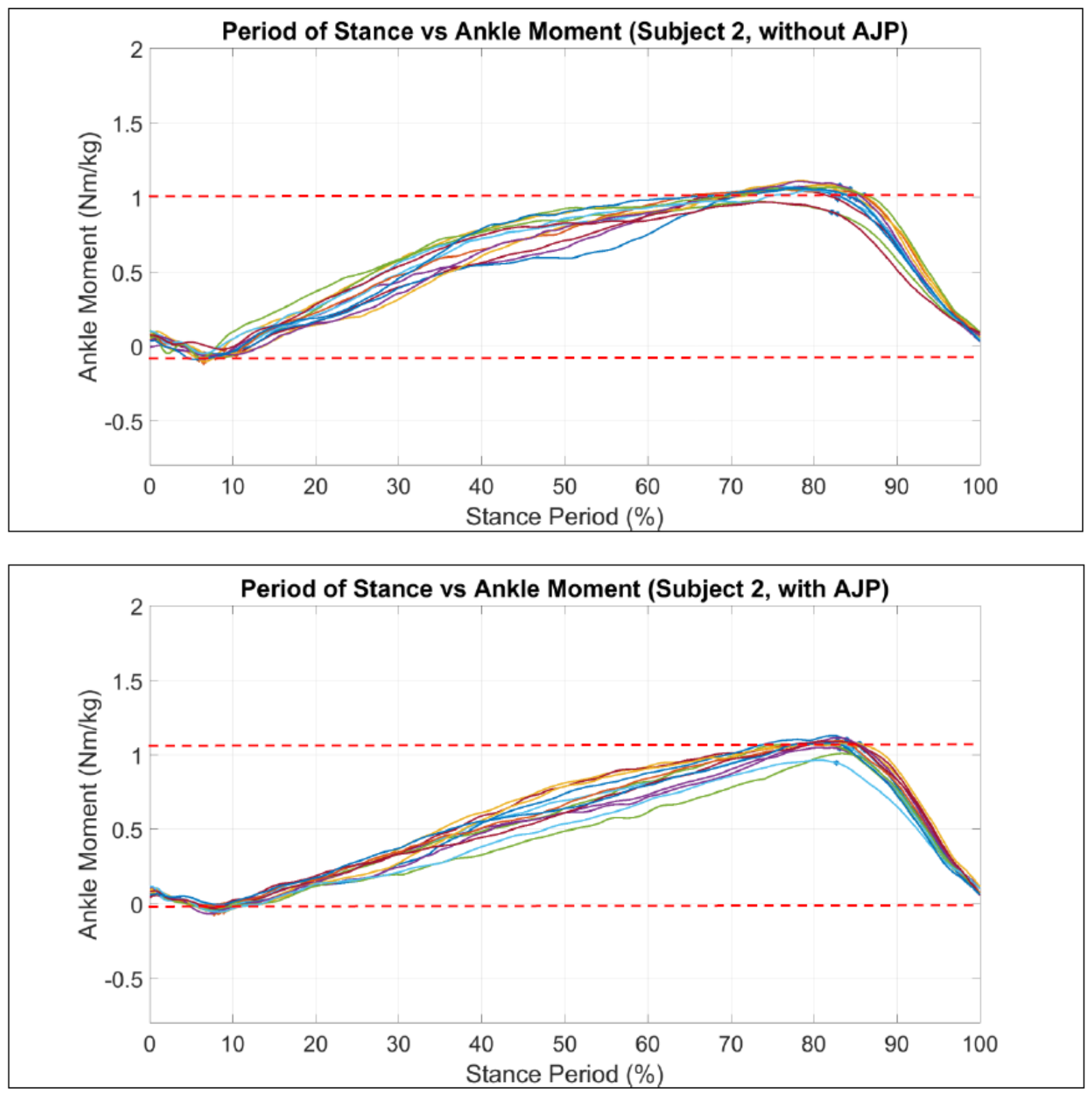

Figure 10.2: Period of Stance vs Ankle Moment of Participant 2.

This figure displays the profile of ankle moment against the period of stance of the CON tests (top) and EXP tests (bottom) of Participant 2. The horizontal dashed red lines indicate the mean peak moment during CPF and CDF for each dataset. Note that due to the removal of outliers identified during the statistical analysis of the data, both the CON and the EXP tests only contain 13 samples.

\subsubsection{Participant 1}

Initial review of the data recorded during the testing of Participant 1 revealed no obvious outliers. As such, 15 samples for both the CON and EXP tests were filtered using the MATLAB code and statistically analysed using SPSS. Statistical analysis revealed that of the CON tests, sample 
2 and 4 contained statistical outliers (walking velocity and peak dorsiflexion as a \% of stance, respectively). As such, the sample size of the CON tests was reduced to 13 samples, whilst the EXP tests remained at 15 samples.

Table 10.2: Temporal-Spatial Results and Analysis of Participant 1.

\begin{tabular}{|c|c|c|c|c|c|c|}
\hline & \multicolumn{2}{|c|}{ Control $(n=13)$} & \multicolumn{2}{|c|}{ Experiment $(n=15)$} & \multicolumn{2}{|c|}{ Impact of AJP } \\
\hline & $\begin{array}{l}\text { Mean } \\
(95 \% \mathrm{Cl})\end{array}$ & $\begin{array}{c}\text { S-W Norm. Test } \\
\text { p-value }\end{array}$ & $\begin{array}{l}\text { Mean } \\
(95 \% \mathrm{Cl})\end{array}$ & $\begin{array}{l}\text { S-W Norm. Test } \\
\text { p-value }\end{array}$ & $\begin{array}{c}\text { Mean Change } \\
(95 \% \mathrm{Cl})\end{array}$ & $\begin{array}{c}\text { Ind. T-Test }{ }^{b} \\
\text { (t-value) p-value }\end{array}$ \\
\hline Stride Length (m) & $\begin{array}{c}1.23 \\
(1.21,1.26)\end{array}$ & 0.994 & $\begin{array}{c}1.27 \\
(1.24,1.29)\end{array}$ & 0.826 & $\begin{array}{c}+2.83 \% \\
(+0.19 \%,+5.47 \%)\end{array}$ & $\begin{array}{c}(2.204) \\
0.037\end{array}$ \\
\hline Walking Velocity $(\mathrm{m} / \mathrm{s})$ & $\begin{array}{c}0.73 \\
(0.70,0.77)\end{array}$ & 0.014 & $\begin{array}{c}0.81 \\
(0.76,0.86)\end{array}$ & 0.011 & $\begin{array}{c}+10.75 \% \\
(+2.36 \%,+19.13 \%)\end{array}$ & $N / A$ \\
\hline $\begin{array}{l}\text { Peak Plantarflexion } \\
\text { (as a \% of stance phase) }\end{array}$ & $\begin{array}{c}7.84 \\
(6.92,8.76)\end{array}$ & 0.305 & $\begin{array}{c}7.78 \\
(7.31,8.25)\end{array}$ & 0.523 & $\begin{array}{c}-0.78 \% \\
(-12.77 \%,+11.22 \%)\end{array}$ & $\begin{array}{c}(-0.133) \\
0.895\end{array}$ \\
\hline $\begin{array}{l}\text { Peak Dorsiflexion } \\
\text { (as a \% of stance phase) }\end{array}$ & $\begin{array}{c}83.06 \\
(82.17,83.96)\end{array}$ & 0.523 & $\begin{array}{c}83.47 \\
(82.88,84.06)\end{array}$ & 0.123 & $\begin{array}{c}+0.49 \% \\
(-0.70 \%,+1.68 \%)\end{array}$ & $\begin{array}{c}(0.844) \\
0.406\end{array}$ \\
\hline
\end{tabular}

${ }^{(a)}$ Shapiro-Wilk (S-W) test to examine normality of dataset. Bold values indicate significant evidence of normality $(p>0.05)$.

$\left.{ }^{b}\right)$ Independent $T$-test to determine if experimental mean is statistically significantly different from control mean. Bold values indicate significant evidence of change $(p<0.05)$.

(c) Increase/decrease of mean experiment value from mean control value (\%).

(*) Levene's Test for Equality of Variance indicates that equal variance cannot be assumed.

Table 10.3: Kinetic and Kinematic Results and Analysis of Participant 1.

\begin{tabular}{|c|c|c|c|c|c|c|}
\hline & \multicolumn{2}{|c|}{ Control $(n=13)$} & \multicolumn{2}{|c|}{ Experiment $(n=15)$} & \multicolumn{2}{|c|}{ Impact of AJP } \\
\hline & Mean $(95 \% \mathrm{Cl})$ & $\begin{array}{c}\text { S-W Norm. Test }{ }^{\mathrm{a}} \\
\text { p-value }\end{array}$ & Mean $(95 \% \mathrm{Cl})$ & $\begin{array}{c}\text { S-W Norm. Test }{ }^{\mathrm{a}} \\
\text { p-value }\end{array}$ & $\begin{array}{c}\text { Mean Change }{ }^{c} \\
(95 \% \mathrm{Cl})\end{array}$ & $\begin{array}{c}\text { Ind. T-Test b } \\
\text { (t-value) p-value }\end{array}$ \\
\hline & \multicolumn{6}{|c|}{ Controlled Dorsiflexion (CDF) } \\
\hline Peak Torque (Nm/kg) & $\begin{array}{c}0.99 \\
(0.95,1.04)\end{array}$ & 0.673 & $\begin{array}{c}1.05 \\
(1.03,1.07)\end{array}$ & 0.954 & $\begin{array}{c}+5.93 \% \\
(+1.25 \%,+10.62 \%)\end{array}$ & $\begin{array}{c}(2.654)^{*} \\
0.016\end{array}$ \\
\hline Peak Angle ( $\left.{ }^{\circ}\right)$ & $\begin{array}{c}5.21 \\
(4.88,5.54)\end{array}$ & 0.129 & $\begin{array}{c}12.09 \\
(11.77,12.42)\end{array}$ & 0.984 & $\begin{array}{c}+132.07 \% \\
(+123.55 \%,+140.59 \%)\end{array}$ & $\begin{array}{l}(31.874) \\
<0.001\end{array}$ \\
\hline \multirow[t]{2}{*}{ 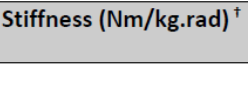 } & $\begin{array}{c}10.99 \\
(10.37,11.61) \\
\end{array}$ & 0.011 & $\begin{array}{c}4.99 \\
(4.82,5.17) \\
\end{array}$ & 0.033 & $\begin{array}{c}-54.58 \% \\
(-49.36 \%,-60.36 \%) \\
\end{array}$ & $N / A$ \\
\hline & \multicolumn{6}{|c|}{ Controlled Plantarflexion (CPF) } \\
\hline Peak Torque (Nm/kg) & $\begin{array}{c}-0.06 \\
(-0.08,-0.05)\end{array}$ & 0.737 & $\begin{array}{c}-0.02 \\
(-0.03,-0.01)\end{array}$ & 0.734 & $\begin{array}{c}-66.69 \% \\
(-40.82 \%,-92.56 \%)\end{array}$ & $\begin{array}{l}(5.408)^{*} \\
<0.001\end{array}$ \\
\hline Peak Angle $\left({ }^{\circ}\right)$ & $\begin{array}{c}-9.40 \\
(-9.95,-8.85)\end{array}$ & 0.503 & $\begin{array}{c}-11.45 \\
(-12.28,-10.62) \\
\end{array}$ & 0.846 & $\begin{array}{c}+21.77 \% \\
(+11.36 \%,+32.18 \%)\end{array}$ & $\begin{array}{l}(-4.299) \\
<0.001\end{array}$ \\
\hline 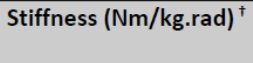 & $\begin{array}{c}-0.39 \\
(-0.49,-0.30)\end{array}$ & 0.873 & $\begin{array}{c}-0.11 \\
(-0.14,-0.07)\end{array}$ & 0.996 & $\begin{array}{c}-73.34 \% \\
(-47.51 \%,-99.18 \%)\end{array}$ & $\begin{array}{l}(6.053)^{*} \\
<0.001\end{array}$ \\
\hline
\end{tabular}

${ }^{(a)}$ Shapiro-Wilk (S-W) test to examine normality of dataset. Bold values indicate significant evidence of normality $(p>0.05)$.

$\left.{ }^{b}\right)$ Independent $T$-test to determine if experimental mean is statistically significantly different from control mean. Bold values indicate significant evidence of change $(p<0.05)$.

(c) Increase/decrease of mean experiment value from control value (\%).

(*) Levene's Test for Equality of Variance indicates that equal variance cannot be assumed.

(') Calculated as per Equation 8.3, using the peak phase angle and its corresponding torque. 


\subsubsection{Participant 2}

Initial review of the data recorded during the testing of Participant 2 revealed an obvious outlier in the CON test (trial 3, wherein the participant made double contact with the force plate). As such, 14 samples for the CON test and 15 samples for the EXP tests were filtered using the MATLAB code and statistically analysed using SPSS. Statistical analysis revealed a further 1 statistical outliers in the CON tests (trial 5; peak plantarflexion as a \% of stance), and 2 staastical outliers in the EXP test (trial 2 and 3; walking velocity outliers). As such the sample size of the EXP tests was reduced to 13 samples.

Table 10.4: Temporal-Spatial Results and Analysis of Participant 2.

\begin{tabular}{|c|c|c|c|c|c|c|}
\hline & \multicolumn{2}{|c|}{ Control $(n=13)$} & \multicolumn{2}{|c|}{ Experiment $(n=13)$} & \multicolumn{2}{|c|}{ Impact of AJP } \\
\hline & $\begin{array}{l}\text { Mean } \\
(95 \% \mathrm{Cl})\end{array}$ & $\begin{array}{c}\text { S-W Norm. Test } \\
\text { p-value }\end{array}$ & $\begin{array}{l}\text { Mean } \\
(95 \% \mathrm{Cl})\end{array}$ & $\begin{array}{l}\text { S-W Norm. Test } \\
\text { p-value }\end{array}$ & $\begin{array}{l}\text { Mean Change } \\
\qquad(95 \% \mathrm{Cl})\end{array}$ & $\begin{array}{c}\text { Ind. T-Test }{ }^{b} \\
\text { (t-value) p-value }\end{array}$ \\
\hline Stride Length (m) & $\begin{array}{c}1.24 \\
(1.21,1.28)\end{array}$ & 0.742 & $\begin{array}{c}1.24 \\
(1.20,1.27)\end{array}$ & 0.743 & $\begin{array}{c}-0.067 \% \\
(-4.51 \%,+3.16 \%)\end{array}$ & $\begin{array}{c}(-0.362) \\
0.721\end{array}$ \\
\hline Walking Speed $(\mathrm{m} / \mathrm{s})$ & $\begin{array}{c}0.79 \\
(0.75,0.84)\end{array}$ & 0.102 & $\begin{array}{c}0.82 \\
(0.80,0.85)\end{array}$ & 0.061 & $\begin{array}{c}+2.41 \% \\
(-1.58 \%,+6.46 \%)\end{array}$ & $\begin{array}{c}(1.247)^{*} \\
0.228\end{array}$ \\
\hline $\begin{array}{l}\text { Peak Plantarflexion } \\
\text { (as a \% of stance phase) }\end{array}$ & $\begin{array}{c}8.01 \\
(7.01,9.01)\end{array}$ & 0.288 & $\begin{array}{c}8.41 \\
(8.08,8.73)\end{array}$ & 0.934 & $\begin{array}{c}+6.04 \% \\
(-7.95 \%,+17.87 \%)\end{array}$ & $\begin{array}{c}(0.821)^{*} \\
0.425\end{array}$ \\
\hline $\begin{array}{l}\text { Peak Dorsiflexion } \\
\text { (as a \% of stance phase) }\end{array}$ & $\begin{array}{c}83.53 \\
(82.61,84.46)\end{array}$ & 0.773 & $\begin{array}{c}84.55 \\
(83.83,85.28)\end{array}$ & 0.263 & $\begin{array}{c}+1.22 \% \\
(-0.11 \%,+2.56 \%)\end{array}$ & $\begin{array}{c}(1.894) \\
0.070\end{array}$ \\
\hline
\end{tabular}

${ }^{(a)}$ Shapiro-Wilk (S-W) test to examine normality of dataset. Bold values indicate significant evidence of normality $(p>0.05)$.

$\left.{ }^{b}\right)$ Independent $T$-test to determine if experimental mean is statistically significantly different from control mean. Bold values indicate significant evidence of change $(p<0.05)$.

(c) Increase/decrease of mean experiment value from mean control value (\%).

$\left({ }^{*}\right)$ Levene's Test for Equality of Variance indicates that equal variance cannot be assumed. 
Table 10.5: Kinetic and Kinematic Results and Analysis of Participant 2.

\begin{tabular}{|c|c|c|c|c|c|c|}
\hline & \multicolumn{2}{|c|}{ Control $(n=13)$} & \multicolumn{2}{|c|}{ Experiment $(n=13)$} & \multicolumn{2}{|c|}{ Impact of AJP } \\
\hline & Mean $(95 \% \mathrm{Cl})$ & $\begin{array}{c}\text { S-W Norm. Test a } \\
\text { p-value }\end{array}$ & Mean $(95 \% \mathrm{Cl})$ & $\begin{array}{c}\text { S-W Norm. Test }{ }^{a} \\
\text { p-value }\end{array}$ & $\begin{array}{l}\text { Mean Change }{ }^{c} \\
(95 \% \mathrm{Cl})\end{array}$ & $\begin{array}{c}\text { Ind. T-Test b } \\
\text { (t-value) } \mathrm{p} \text {-value }\end{array}$ \\
\hline & \multicolumn{6}{|c|}{ Controlled Dorsiflexion (CDF) } \\
\hline Peak Torque (Nm/kg) & $\begin{array}{c}1.00 \\
(0.97,1.04)\end{array}$ & 0.055 & $\begin{array}{c}1.04 \\
(1.02,1.07)\end{array}$ & 0.547 & $\begin{array}{c}+3.96 \% \\
(+0.03 \%,+7.89 \%)\end{array}$ & $\begin{array}{l}(2.078) \\
0.049\end{array}$ \\
\hline Peak Angle $\left({ }^{\circ}\right)$ & $\begin{array}{c}7.87 \\
(7.38,8.35) \\
\end{array}$ & 0.389 & $\begin{array}{c}15.86 \\
(15.66,16.05) \\
\end{array}$ & 0.884 & $\begin{array}{c}+101.53 \% \\
(+95.22 \%,+107.84 \%) \\
\end{array}$ & $\begin{array}{l}(33.216) \\
<0.001\end{array}$ \\
\hline \multirow[t]{2}{*}{ Stiffness $(\mathrm{Nm} / \mathrm{kg} \cdot \mathrm{rad})^{\dagger}$} & $\begin{array}{c}7.38 \\
(6.87,7.89)\end{array}$ & 0.539 & $\begin{array}{c}3.77 \\
(3.67,3.88)\end{array}$ & 0.812 & $\begin{array}{c}-48.88 \% \\
(-41.90 \%,-55.87 \%)\end{array}$ & $\begin{array}{c}(-15.130)^{*} \\
<0.001\end{array}$ \\
\hline & \multicolumn{6}{|c|}{ Controlled Plantarflexion (CPF) } \\
\hline Peak Torque (Nm/kg) & $\begin{array}{c}-0.06 \\
(-0.07,-0.05)\end{array}$ & 0.970 & $\begin{array}{c}-0.03 \\
(-0.04,-0.02)\end{array}$ & 0.596 & $\begin{array}{c}-51.68 \% \\
(-25.18 \%,-78.17 \%)\end{array}$ & $\begin{array}{l}(4.026) \\
<0.001\end{array}$ \\
\hline Peak Angle ( $\left(^{\circ}\right)$ & $\begin{array}{c}-6.92 \\
(-7.71,-6.13)\end{array}$ & 0.074 & $\begin{array}{c}-10.54 \\
(-11.69,-9.39)\end{array}$ & 0.978 & $\begin{array}{c}+52.35 \% \\
(+33.23 \%,+71.47 \%)\end{array}$ & $\begin{array}{l}(-5.650) \\
<0.001\end{array}$ \\
\hline Stiffness $(\mathrm{Nm} / \mathrm{kg} \cdot \mathrm{rad})^{\dagger}$ & $\begin{array}{c}-0.51 \\
(-0.59,-0.42)\end{array}$ & 0.692 & $\begin{array}{c}-0.15 \\
(-0.20,-0.10)\end{array}$ & 0.504 & $\begin{array}{c}-69.98 \% \\
(-51.55 \%,-88.40 \%)\end{array}$ & $\begin{array}{l}(7.839) \\
<0.001\end{array}$ \\
\hline
\end{tabular}

(a) Shapiro-Wilk (S-W) test to examine normality of dataset. Bold values indicate significant evidence of normality ( $p>0.05)$.

$(b)$ Independent $T$-test to determine if experimental mean is statistically significantly different from control mean. Bold values indicate significant evidence of change $(p<0.05)$.

(c) Increase/decrease of mean experiment value from control value (\%).

(*) Levene's Test for Equality of Variance indicates that equal variance cannot be assumed.

$\left(^{+}\right)$Calculated as per Equation 8.3, using the peak phase angle and its corresponding torque.

\subsubsection{Participant 3}

Initial review of the data recorded during the testing of Participant 3 revealed no obvious outliers. However, there was a recording error with one EXP sample and as such only 14 samples for the EXP were filtered using the MATLAB code and statistically analysed using SPSS, whilst the same was done for all 15 CON samples. Statistical analysis revealed 2 statistical outliers in the CON tests (trial 3 and 7; peak plantarflexion as a \% of stance) removed from the dataset. As such the sample size of the CON tests was reduced to 13 samples. 
Table 10.6: Temporal-Spatial Results and Analysis of Participant 3.

\begin{tabular}{|c|c|c|c|c|c|c|}
\hline & \multicolumn{2}{|c|}{ Control $(n=13)$} & \multicolumn{2}{|c|}{ Experiment $(n=14)$} & \multicolumn{2}{|c|}{ Impact of AJP } \\
\hline & $\begin{array}{l}\text { Mean } \\
(95 \% \mathrm{Cl})\end{array}$ & $\begin{array}{l}\text { S-W Norm. Test a } \\
\text { p-value }\end{array}$ & $\begin{array}{l}\text { Mean } \\
(95 \% \mathrm{Cl})\end{array}$ & $\begin{array}{l}\text { S-W Norm. Test a } \\
\text { p-value }\end{array}$ & $\begin{array}{l}\text { Mean Change } \\
\qquad(95 \% \mathrm{Cl})\end{array}$ & $\begin{array}{c}\text { Ind. T-Test }{ }^{\text {b }} \\
\text { (t-value) p-value }\end{array}$ \\
\hline Stride Length (m) & $\begin{array}{c}1.39 \\
(1.31,1.46)\end{array}$ & 0.874 & $\begin{array}{c}1.33 \\
(1.30,1.37)\end{array}$ & 0.763 & $\begin{array}{c}-4.00 \% \\
(-9.44 \%,-1.45 \%)\end{array}$ & $\begin{array}{c}(-1.514) \\
0.143\end{array}$ \\
\hline Walking Speed (m/s) & $\begin{array}{c}1.02 \\
(0.97,1.07)\end{array}$ & 0.033 & $\begin{array}{c}0.97 \\
(0.94,1.00)\end{array}$ & 0.039 & $\begin{array}{c}-4.39 \% \\
(-9.65 \%,+0.89 \%)\end{array}$ & $N / A$ \\
\hline $\begin{array}{l}\text { Peak Plantarflexion } \\
\text { (as a \% of stance phase) }\end{array}$ & $\begin{array}{c}8.73 \\
(8.42,9.06)\end{array}$ & 0.644 & $\begin{array}{c}7.36 \\
(6.68,8.05)\end{array}$ & 0.359 & $\begin{array}{c}-15.67 \% \\
(-7.94 \%,-24.60 \%)\end{array}$ & $\begin{array}{c}(-4.106)^{*} \\
<0.685\end{array}$ \\
\hline $\begin{array}{l}\text { Peak Dorsiflexion } \\
\text { (as a \% of stance phase) }\end{array}$ & $\begin{array}{c}83.26 \\
(82.36,84.15)\end{array}$ & 0.686 & $\begin{array}{c}83.41 \\
(82.78,84.07)\end{array}$ & 0.673 & $\begin{array}{c}+0.19 \% \\
(-0.94 \%,+1.40 \%)\end{array}$ & $\begin{array}{l}(0.411) \\
0.685\end{array}$ \\
\hline
\end{tabular}

${ }^{(a)}$ Shapiro-Wilk (S-W) test to examine normality of dataset. Bold values indicate significant evidence of normality $(p>0.05)$.

${ }^{b}$ ) Independent $T$-test to determine if experimental mean is statistically significantly different from control mean. Bold values indicate significant evidence of change $(p<0.05)$.

(c) Increase/decrease of mean experiment value from mean control value (\%).

$\left(^{*}\right)$ Levene's Test for Equality of Variance indicates that equal variance cannot be assumed.

Table 10.7: Kinetic and Kinematic Results and Analysis of Participant 3.

\begin{tabular}{|c|c|c|c|c|c|c|}
\hline & \multicolumn{2}{|c|}{ Control $(n=13)$} & \multicolumn{2}{|c|}{ Experiment $(n=14)$} & \multicolumn{2}{|c|}{ Impact of AJP } \\
\hline & Mean $(95 \% \mathrm{Cl})$ & $\begin{array}{c}\text { S-W Norm. Test a } \\
\text { p-value }\end{array}$ & Mean $(95 \% \mathrm{Cl})$ & $\begin{array}{l}\text { S-W Norm. Test a } \\
\text { p-value }\end{array}$ & $\begin{array}{l}\text { Mean Change }{ }^{\circ} \\
(95 \% \mathrm{Cl})\end{array}$ & $\begin{array}{c}\text { Ind. T-Test }{ }^{\text {b }} \\
\text { (t-value) } p \text {-value }\end{array}$ \\
\hline & \multicolumn{6}{|c|}{ Controlled Dorsiflexion (CDF) } \\
\hline Peak Torque (Nm/kg) & $\begin{array}{c}1.17 \\
(1.12,1.22)\end{array}$ & 0.074 & $\begin{array}{c}1.23 \\
(1.21,1.25)\end{array}$ & 0.816 & $\begin{array}{c}+4.71 \% \\
(-0.27 \%,+8.63 \%)\end{array}$ & $\begin{array}{c}(1.988)^{*} \\
0.064\end{array}$ \\
\hline Peak Angle $\left({ }^{\circ}\right)$ & $\begin{array}{c}9.13 \\
(8.61,9.64)\end{array}$ & 0.362 & $\begin{array}{c}16.40 \\
(16.11,16.69)\end{array}$ & 0.256 & $\begin{array}{c}+79.66 \% \\
(+73.76 \%,+85.11 \%)\end{array}$ & $\begin{array}{l}(28.834) \\
<0.001\end{array}$ \\
\hline \multirow[t]{2}{*}{ 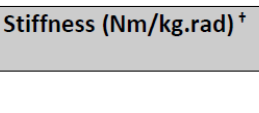 } & $\begin{array}{c}7.39 \\
(7.01,7.76)\end{array}$ & 0.288 & $\begin{array}{c}4.29 \\
(4.21,4.37)\end{array}$ & 0.481 & $\begin{array}{c}-41.93 \% \\
(-37.43 \%,-46.98 \%)\end{array}$ & $\begin{array}{c}(-19.055)^{*} \\
<0.001\end{array}$ \\
\hline & \multicolumn{6}{|c|}{ Controlled Plantarflexion (CPF) } \\
\hline Peak Torque (Nm/kg) & $\begin{array}{c}-0.07 \\
(-0.09,-0.06)\end{array}$ & 0.285 & $\begin{array}{c}-0.01 \\
(-0.02,0.00)\end{array}$ & 0.769 & $\begin{array}{c}-84.23 \% \\
(-64.70 \%,-103.96 \%)\end{array}$ & $\begin{array}{l}(8.984)^{*} \\
<0.001\end{array}$ \\
\hline Peak Angle $\left(^{\circ}\right)$ & $\begin{array}{c}-5.75 \\
(-6.30,-5.19)\end{array}$ & 0.691 & $\begin{array}{c}-10.19 \\
(-11.16,-9.23)\end{array}$ & 0.664 & $\begin{array}{c}+77.40 \% \\
(+56.97 \%,+94.46 \%)\end{array}$ & $\begin{array}{l}(-8.319) \\
<0.001\end{array}$ \\
\hline 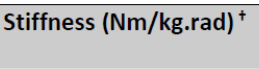 & $\begin{array}{c}-0.74 \\
(-0.86,-0.62)\end{array}$ & 0.942 & $\begin{array}{c}-0.07 \\
(-1.10,-0.03)\end{array}$ & 0.856 & $\begin{array}{c}-90.86 \% \\
(-73.85 \%,-105.38 \%)\end{array}$ & $\begin{array}{c}(12.088)^{*} \\
<0.001\end{array}$ \\
\hline
\end{tabular}

${ }^{(a)}$ Shapiro-Wilk (S-W) test to examine normality of dataset. Bold values indicate significant evidence of normality $(p>0.05)$.

(b) Independent $T$-test to determine if experimental mean is statistically significantly different from control mean. Bold values indicate significant evidence of change $(p<0.05)$.

(c) Increase/decrease of mean experiment value from control value (\%).

$\left({ }^{*}\right)$ Levene's Test for Equality of Variance indicates that equal variance cannot be assumed.

$\left.{ }^{+}\right)$Calculated as per Equation 8.3, using the peak phase angle and its corresponding torque. 


\section{Chapter 11}

\section{Discussion}

\subsection{Summary of Results}

The AJP produced for this study was designed with the intention of increasing sagittal plane rotation of a baseline AFP (Otto Bock 1D10) relative to a pseudo tibia during stance, with a controlled moment response that is specifically designed to replicate natural human stance mechanics. As outlined earlier, the experimental methodology runs two sets of trials on each participant, a CON and EXP test. The CON test uses a DPP which contains a deactivated AJP, whilst the EXP test uses a PP which contains an activated AJP (see Section 9.2.3). Thus the intention of this experimental analysis is to define this AJP impact on a baseline AFP (Otto Bock 1D10, a SACH-Dynamic foot) using quantitative metrics. The analysis steers clear of qualitative metrics (comfort, balance, ease of walking) due to the implications that arise when attempting to define qualitative outcomes using a participant population of non-amputees and a simulated amputee gait cycle. The methodology for this study was additionally designed with the intention to control TS variables in order to produce a reliable analysis of the impact that the AJP has on the KK variables of stance. Figure 11.1 graphically displays the summarised changes of kinetic and kinematics stance metrics that each participant displays when utilising the activated AJP (i.e. the increase/decrease in variable value with the PP in comparison to the DPP control). Figure 11.2 likewise displays the summarised changes in TS metrics.

The analysis of the results begins here with a discussion on the KK results. The specific phases of focus are CPF and CDF of the simulated gait cycle, and thus each of these two phases requires a pre-definition of what an improvement would be defined as.

For the CDF phase, an improvement in the peak moment and joint angle would be evident from a stable or slight decrease in peak moment magnitude and an increase in peak angle. An increase in peak angle would be indicative of easier forefoot rollover during mid-to-late stance, whilst a slight decrease in peak moment would be attributed to the prevention of GRF CoP forward progression during forefoot rollover due to the relative reduction in ankle stiffness. Improvements for both of these metrics would be indicative of an ability to maintain a flat foot during forward BW progression, which is the most stable position during single limb support [Wagner et al., 1987; 


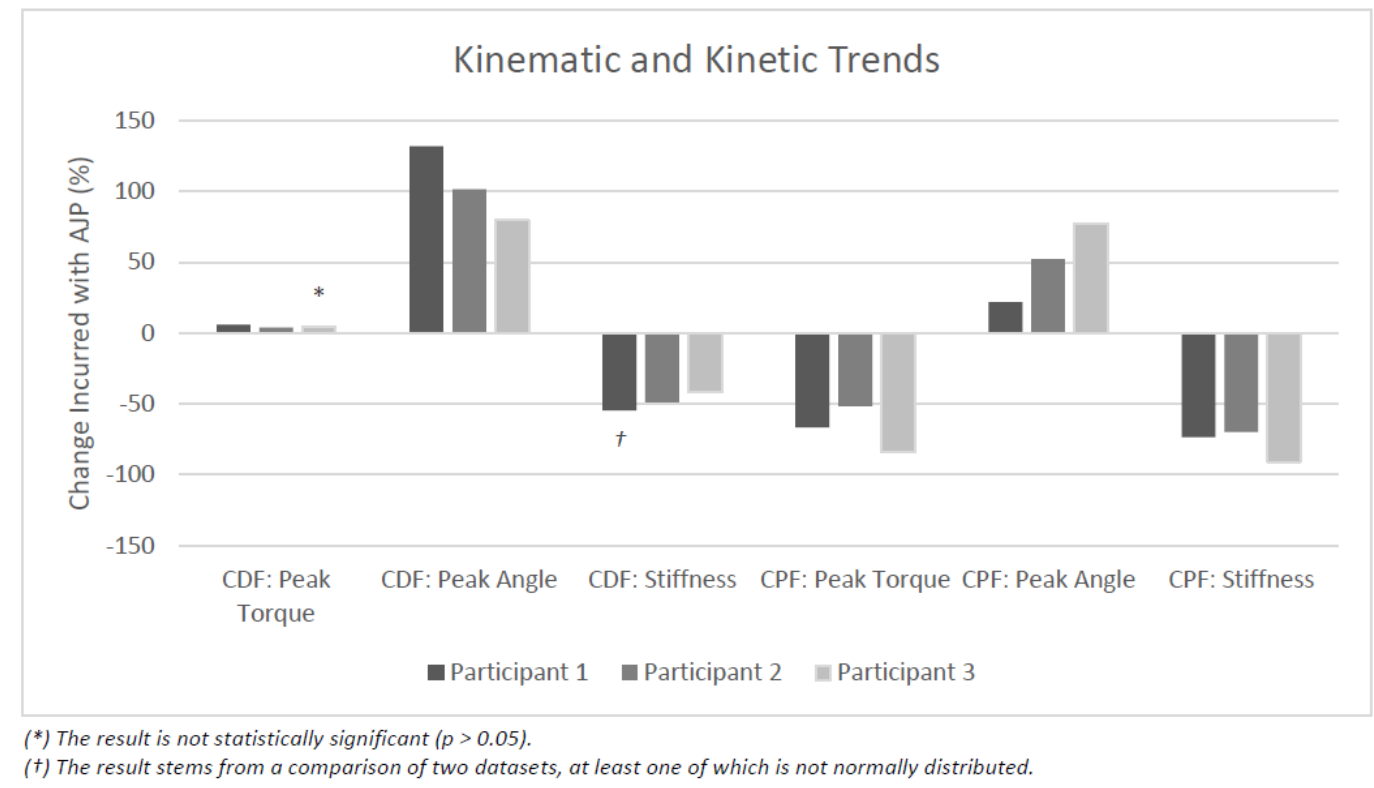

Figure 11.1: Kinetic and Kinematic Changes and Trends.

This figure graphically displays the changes in the kinetic and kinematic variables that are of focus during the stance phase of the simulated amputee gait cycle when using an activated AJP. It also provides an ability to analyse the trends across all three participants, and to determine whether this change is considered to be evident of a 'positive' response.

Perry et al., 1997]. These changes were observed in [Su et al., 2010] when a flexion unit was added to a baseline foot. The combination of a slight decrease in moment profile (or else stable) and a large increase in joint RoM would thus result in a decrease in the ankle stiffness metric (Equation 9.3).

Analysing CDF trends across the three participants reveals that activating the AJP yields a consistent marginal impact on peak moment, increasing the variable by $6 \%, 4 \%$ and $5 \%$ for each participant respectively. This result was statistically significant for only Participant $1(p=0.016)$ and $2(p=0.049)$, whilst Participant 3's $(p=0.064)$ experienced a non-significant increase between CON and EXP trials. Further, the activation of the AJP increased the ankle joint angle RoM during this phase by $132 \%, 102 \%$ and $80 \%$ for each participant respectively, providing a dorsiflexion termination angle of $12^{\circ}, 16^{\circ}$ and $16^{\circ}$ respectively, as opposed to the $5^{\circ}, 8^{\circ}$ and $6^{\circ}$ angle observed when relying on the baseline AFP (i.e. using the deactivated AJP). This is an important result as the approximate doubling of RoM indicates that the AJP is allowing for forward tibial progression over a flat foot for an extended period of time, akin to natural mechanics, and thus provides an increased period of maximum stability during tibial progression. Additional verification of this positive result displayed in Figure 11.3, where it is evident that during CDF the AJP allows the baseline AFP foot to remain in flat contact with the ground, whilst when reliant on the baseline AFP for forefoot mobility the participant is forced into early heel rise that indicates a rigid forefoot rollover. Further, observe how the change in occurrence of CDF termination as a percentage of a complete stance phase is statistically insignificant for all participants $(p>0.05)$. 


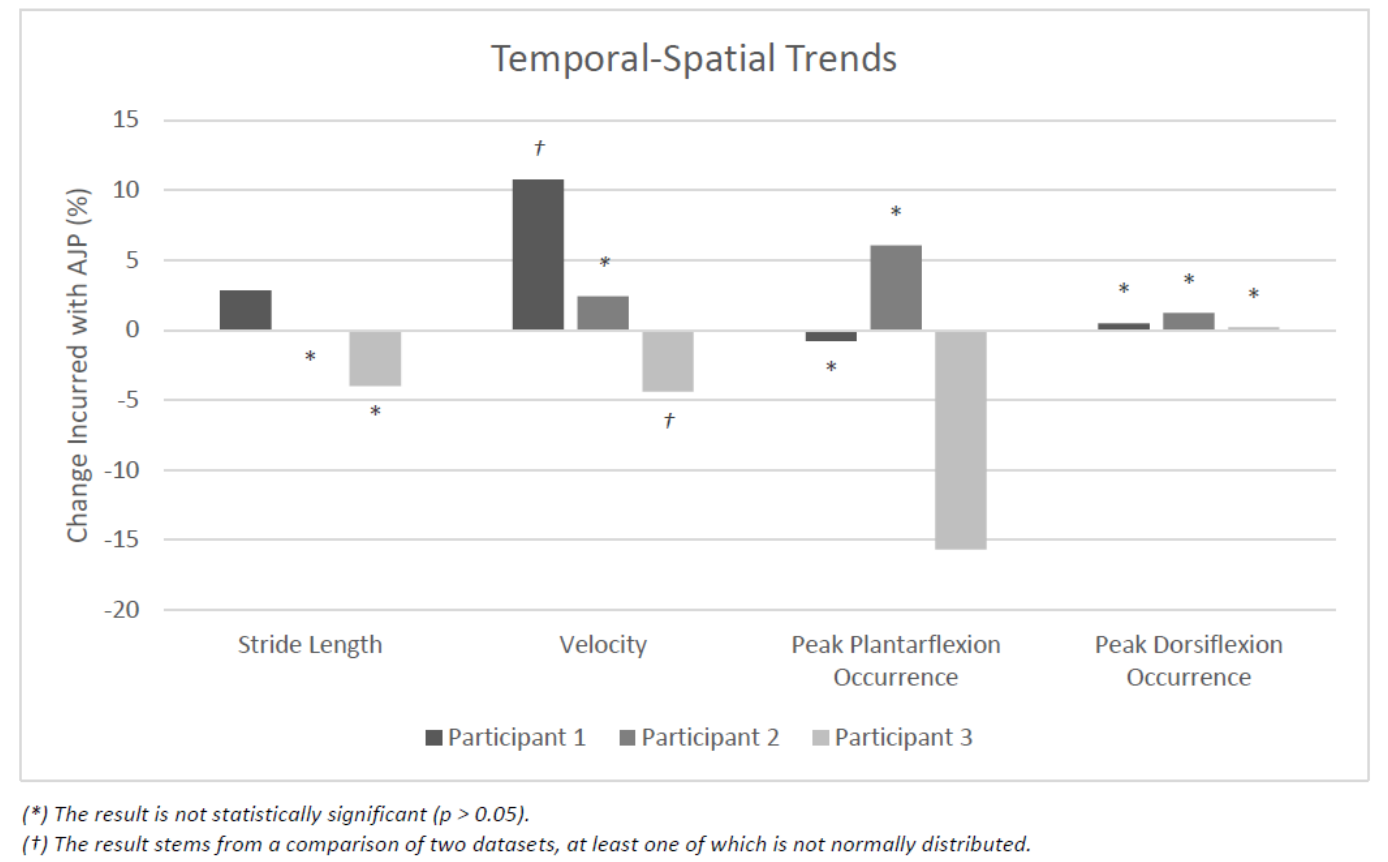

Figure 11.2: Temporal-Spatial Changes and Trends.

This figure graphically displays the changes in the temporal spatial variables that are of focus during the stance phase of the simulated amputee gait cycle when using an activated AJP. It also provides an ability to analyse the trends across all three participants, and to determine whether this change is considered to be evident of a 'positive' response.

Whilst a slight decrease in ankle moment was expected, there are a variety of factors that influence this change (e.g. GRF magnitude, accuracy of CoP measurement etc.), and a relatively small increase $(>10 \%)$ can actually be considered stable when considering the magnitude of increase in joint RoM. The combination of a stable moment profile and the more doubling of joint RoM during CDF results in the decrease in ankle stiffness response by $55 \%, 49 \%$ and $42 \%$ for each participant respectively, approximately halving the resistance to forward tibial progression for all participants. Note however that whilst this result was statistically significant for Participant 2 and $3(p<0.001)$, both the CON and EXP datasets of Participant 1 were not normally distributed and thus the difference in means was marked as $\mathrm{N} / \mathrm{a}$. This is problematic, and is perhaps evidence of an insufficient number of CON and EXP trials.

With regards to the CPF phase, a successful change to peak moment and joint angle would be evident from a decrease in peak moment and an increase in angle at phase termination. This decrease in peak moment is expected due to the improved weight acceptance mechanism and accelerated transition to footflat that the plantarflexion mobility of the AJP provides, i.e. the impact energy of the GRF is absorbed by the springs and is converted into a driving plantarflexion response in the the baseline AFP. This would thus result in a decrease in ankle stiffness. Results show that the peak moment decreased by $67 \%, 52 \%$ and $84 \%$ for each participant respectively, which for all participants was statistically significant $(p<0.001)$. Further, the peak angle during 

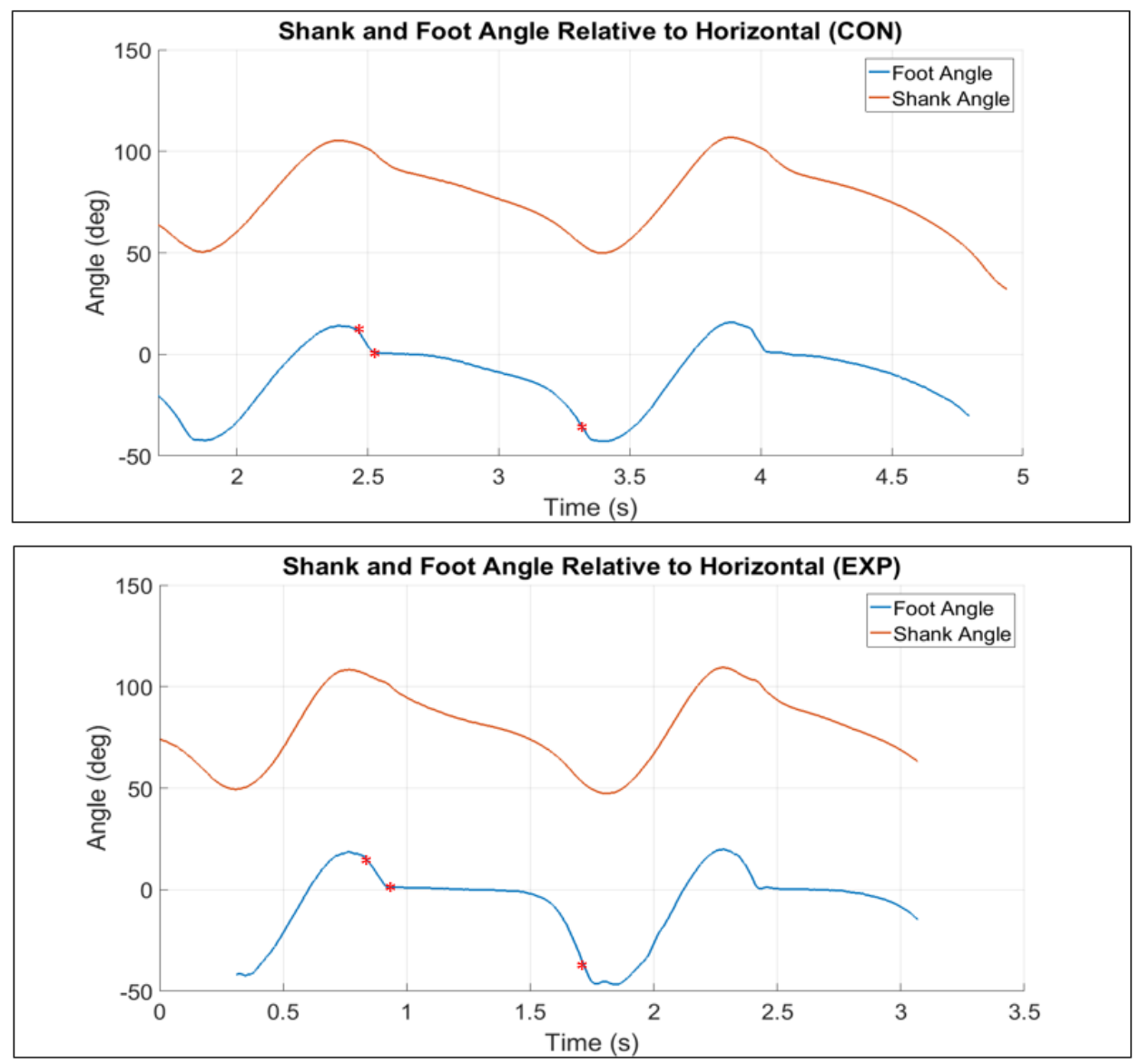

Figure 11.3: Foot Angle during Stance.

This figure graphically displays the foot and shank angle of a single trial from Participant 2's CON (top) and EXP (bottom) datasets. The stars indicate the initiation of (from left to right) heelstrike, footflat and toe-off respectively. The figure serves as a method of analysing the variation duration of footflat with and without the activate AJP. Observe how the shape of the shank angle curve of the CON trial is approximately similar to that of the EXP trial, yet the foot angle differs during stance - specifically during the transition from footflat to toe off. Further, note how the CON foot angle is characterised as a gradual curve of a negative (plantarflexed) angle during this stage, indicating that the heel leaves the ground as the tibia progresses over the ankle. In contrast the EXP trial allows for a significant period of foot flat (when foot angle $=0^{\circ}$ ), and then a high gradient curve of plantarflexion to toe off. This difference in shape is due to the fact that during mid-stance the AJP used in the EXP trial allows for forward tibial progression over a flat foot, whilst the lack of ankle mobility in the CON test forces the participant to rollover the forefoot of the baseline AFP. This is graphical representation of the unnatural rollover that is associated with low cost AFP's. 
this phase increased by $22 \%, 52 \%$ and $77 \%$ to angles of $-11^{\circ},-11^{\circ}$ and $-10^{\circ}$ for each participant respectively ( $p<0.001$ for all participants). This is interesting as although Participant 3 had a larger relative increase in RoM, all three participants displayed a strong correlation of peak angle during the EXP test. This can be attributed to one of two explanations, either that their plantarflexion RoM is limited by footflat (i.e. all participants incur heelstrike with the foot at an angle of $10^{\circ}$ $11^{\circ}$ to the horizontal), or alternatively that the AJP posterior spring set (which is responsible for controlling plantarflexion) only allows for up to $11^{\circ}$ of plantarflexion. Yet, the latter is unlikely as the spring set in question has an allowable deflection of up to $9.8 \mathrm{~mm}$ yielding a potential plantarflexion of $20^{\circ}+$, indicating that the former explanation should be correct. Analysis of the raw data confirmed this by showing that the plantarflexion angle during CPF is indeed physically limited by ground contact. And whilst the same is true of the CON test when the participants used the DPP, analysis of the foot angle relative to the horizontal just before heelstrike shows that participants utilise a heel contact foot angle that is $\pm 5^{\circ}$ more acute, i.e. they utilise a flat(ter) foot ground contact angle as opposed to a natural heelstrike seen in the EXP trials. This indicates that after a period of adjustment to the DPP the participants become aware of the baseline AFP's natural plantarflexion limitations, and subsequently accommodate for the low ankle mobility and stiff CPF transition by pre-emptively utilising a flatter foot contact. This theory is confirmed in literature [Wirta et al., 1991]. The hypothesised rationale behind this is that the participants find the baseline AFP's rollover shape unnatural and uncomfortable, leading them to autonomously utilise a flat foot ground contact that yields a decreased moment through their immobilised limb.

The combined results of an increased RoM and decreased moment yields a $73 \%, 70 \%$ and $91 \%$ decrease in ankle stiffness during this phase for each participant respectively. What is therefore evident from these results is that across all three participants, activating the AJP results in a consistent 'positive' change for all variables of CPF.

Important to analyse is the loop created when plotting ankle moment against the corresponding joint mangle during the stance phase of the simulated gait cycles. These graphs are displayed in Figure 11.4. Immediately evident from Figure11.4 is the difference in consistency of shape between the CON and EXP tests. Observe in the EXP plot that whilst the trial-to-trial magnitude of the termination points (both angle and moment) varies, the gradient of CPF/CDF slope (stiffness) to reach these termination points remains approximately consistent. In contrast the profiles of the CON tests are more irregular, as the the gradients of CPF/CDF slope in the CON tests are inconsistent and the trial-to-trial termination points are scattered.

Further, observe how the CPF gradient appears to have a linear relationship between ankle angle and moment, as was found in the analysis of natural CPF by Palmer [1999] (see Figure 4.1). This indicates that the introduction of the activated AJP produces a reliable and consistent elastic response during CPF, and thus a single trial that imparts a larger peak GRF will produce a angular response that could reliably be predicated according to the gradient (i.e. the $-0.51 \mathrm{Nm} / \mathrm{rad}$.kg CPF stiffness found for Participant 2). Palmer [1999] also found that the CDF elastic response in natural biomechanics does not obey a linear relationship (see Figure 4.1). The same can be observed in both the CON and EXP datasets of this study. However, what is evident in Figure 11.4 is that 
the EXP CDF curve appears to follow two linear lines, the first initiating at peak plantarflexion and terminating at $0^{\circ}$, the second steeper line that initiates at $0^{\circ}$ and terminates at peak dorsiflexion. Note that this second steeper line represents the CDF stiffness of $3.77 \mathrm{Nm} / \mathrm{rad} . \mathrm{kg}$ of Participant 2. This differentiation is thus reflective of the AJP's two separate spring sets, a weaker pseudoplantarflexor set and a stronger pseudo-dorsiflexor set that are active for negative and positive ankle angles respectively. This will be further discussed in Section 11.2.

It is difficult to define the relationship between temporal spatial variables and stance mechanics, but literature indicates that variation of ankle flexion/torsion response has no significant impact on either step length nor walking speed [Su et al., 2010; Lehmann et al., 1993; Powers et al., 1994; Torburn et al., 1990]. Yet when considering the fact that the AJP design utilises compression springs that have loading/compression response that is directly proportional to loading magnitude and would thus yield a greater angular rotation of the baseline AFP relative to the AIB, variables such as body weight, walking velocity and stride length have the potential to impact these stance mechanics. Although it is not an absolute requirement, for the purpose of defining the impact the AJP has on the baseline AFP it is desirable to control these three variables and thus have consistency in intra-participant gait parameters. Thus each participant was encouraged to maintain a consistent walking velocity and stride length through both the EXP and CON tests. The independent T-test conducted on each participants EXP and CON tests in this study would therefore ideally produce stride length and walking velocity datasets that (i) are normally distributed with (ii) have a difference of means that is statistically insignificant. Yet the results (see Tables 10.2, 10.4 and 10.6) show that this is not always the case. Whilst Participant 2's walking velocity results indicate a statistical insignificant difference of means $(p=0.228)$, Participant 1 and Participant 3's CON and EXP datasets indicate non-normal distribution and thus statistical analysis of difference of means using an independent T-test is not applicable (N/a). This is problematic, as for Participant 1 and 3 it adds a potentially non-consistent variable that could impact the analysis of the EXP vs CON improvements. In terms of stride length, Participant $2(p=0.721)$ and 3 's $(p=0.143)$ results showed a difference of means that is statistically insignificant. However, Participant 1's difference of means is shown to be statistically significant, although this margin of increase is small (-4\%). Possible causes of these discrepancies are explored in Section 11.3.

Yet, the outcome of the analysis of TS gait variables is that only Participant 2 yielded statistically insignificant difference of means for both walking velocity and stride length. Visual analysis of the vertical and fore-aft GRF profiles of Participant 2 is displayed in Figures 11.5 and 11.6, which indicate that the CON vs EXP profiles are consistent, although a slight increase in peak-to-peak fore-aft GRF is observed in the EXP test. At present the cause of this increase is not yet clear, especially since there is no significant increase in participant 2's walking velocity or stride length. Possible causes for this include a more natural rollover shape or a more confident stride. Yet the consistency in vertical and fore-aft GRF shape for participant 2 is evidence of the benefits associated with controlling TS variables. However, due to the consistent findings in literature that flexion response of AFP's has no significant effect on walking velocity or stride length, these TS variable inconsistencies do not yield the KK results unverifiable. 

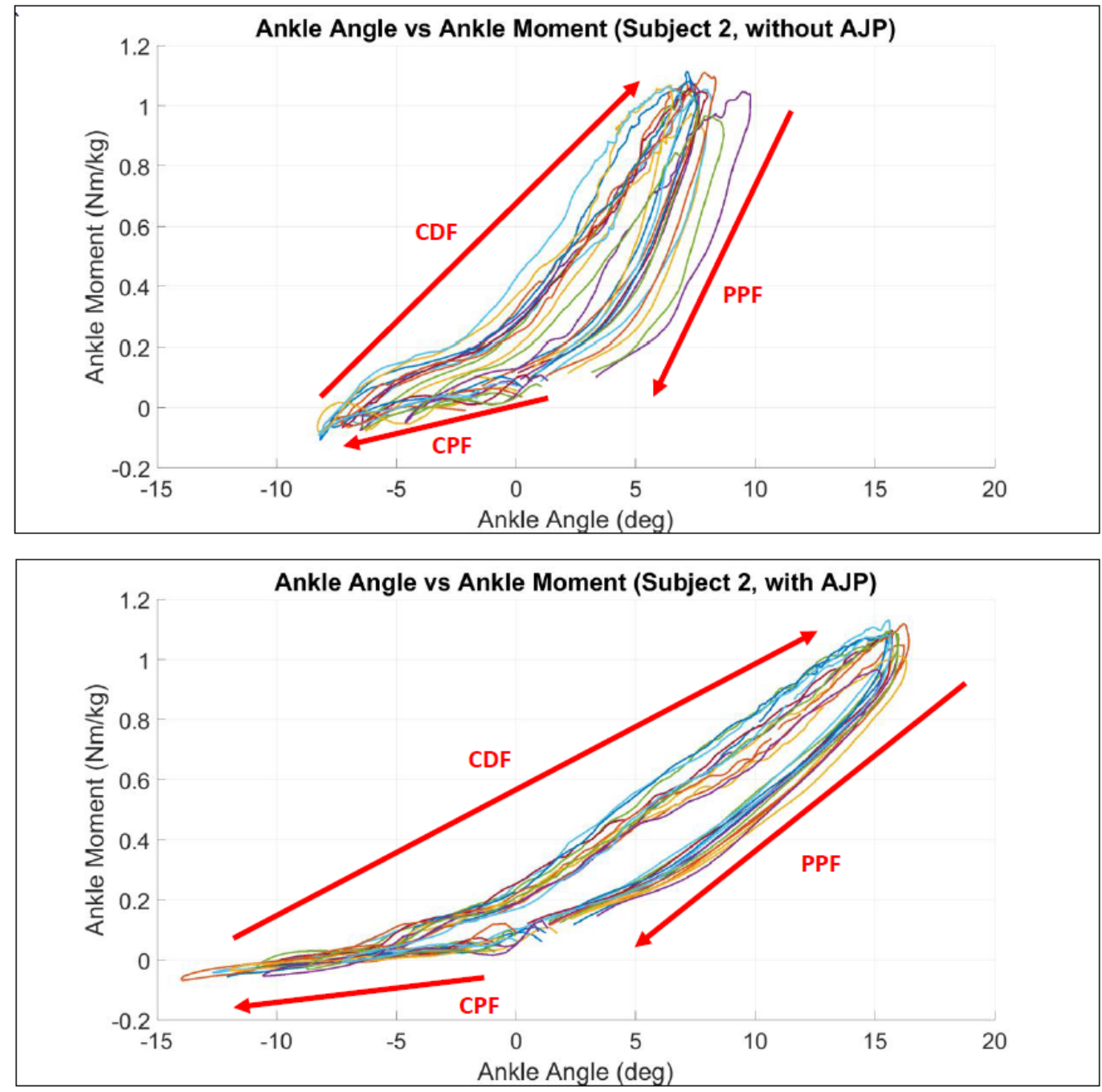

Figure 11.4: Ankle Angle vs Ankle Moment of Participant 2.

This figure displays the profile of ankle joint angle vs moment of the CON datasets (top) and EXP datasets (bottom) of Participant 2. The red arrows are used to indicate the clockwise rotation of these plots, and how the three phases of stance (CPF, CDF, PPF) are defined by different gradients (i.e. stiffness). The use of the term PPF here denotes the unloading of the PP/DPP system but is not a technically accurate description of this phase, as this is the term used when describing an musculature-powered transition to toe off in natural stance mechanics. In this case the phase is actually characterised as a passive unloading of energy stored in the AJP during CDF. 

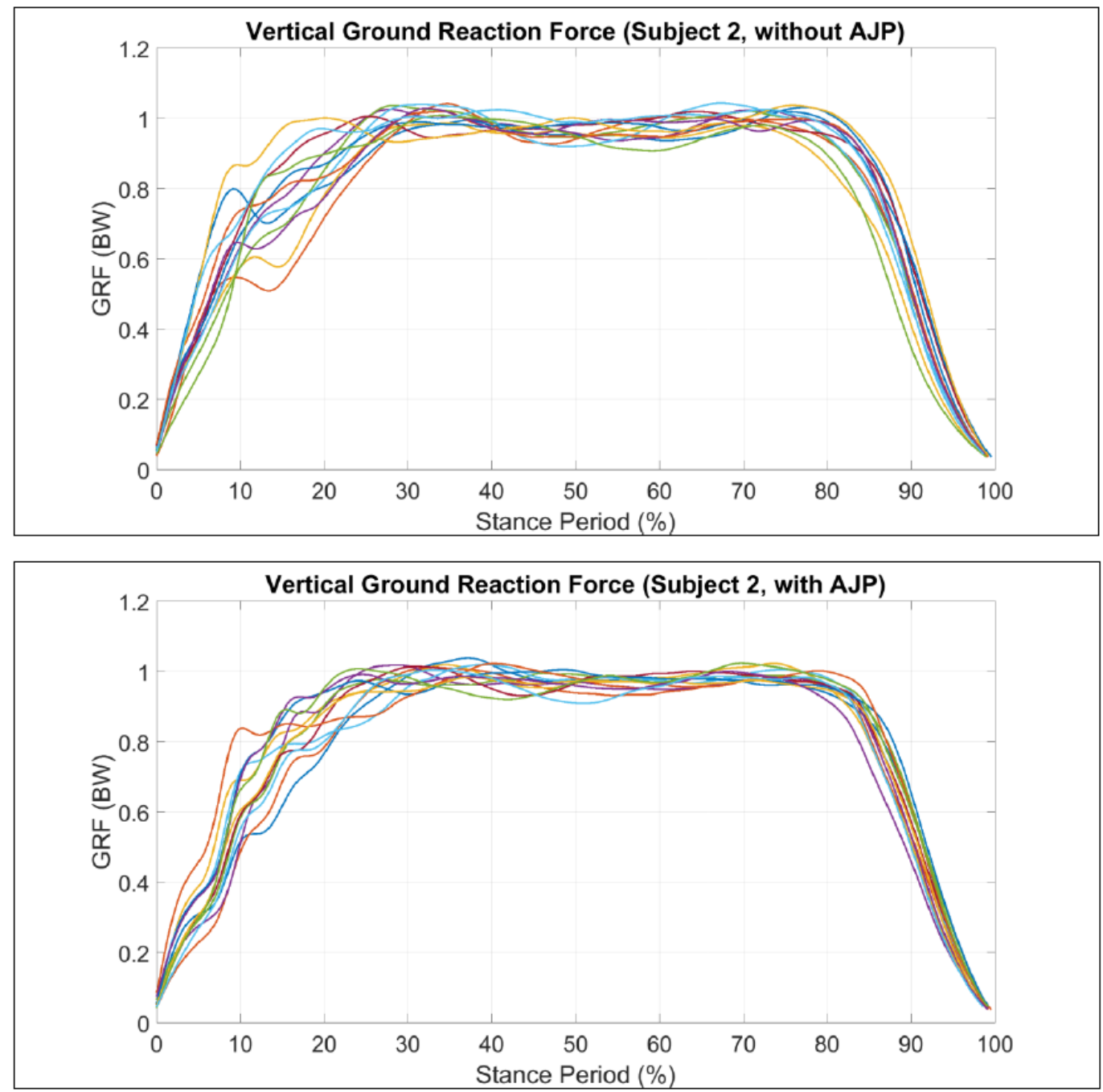

Figure 11.5: Vertical Ground Reaction Force over Period of Stance of Participant 2.

This figure displays the profile of vertical GRF against the period of stance of of the CON tests (top) and EXP tests (bottom) of Participant 2. The graphs reflects that the CON vs EXP vertical GRF profile is consistent. This is expected, as the statistical analysis of the CON vs EXP temporal-spatial variables showed no significant difference of means. This evidence of controlled GRF via the control of temporal-spatial variables in the experimental methodology thus adds further validity to the outcomes of the analysis of the kinetic and kinematic results. Further, note that due to the removal of outliers identified during the statistical analysis of the data, both the CON and the EXP tests only contain 13 samples. 

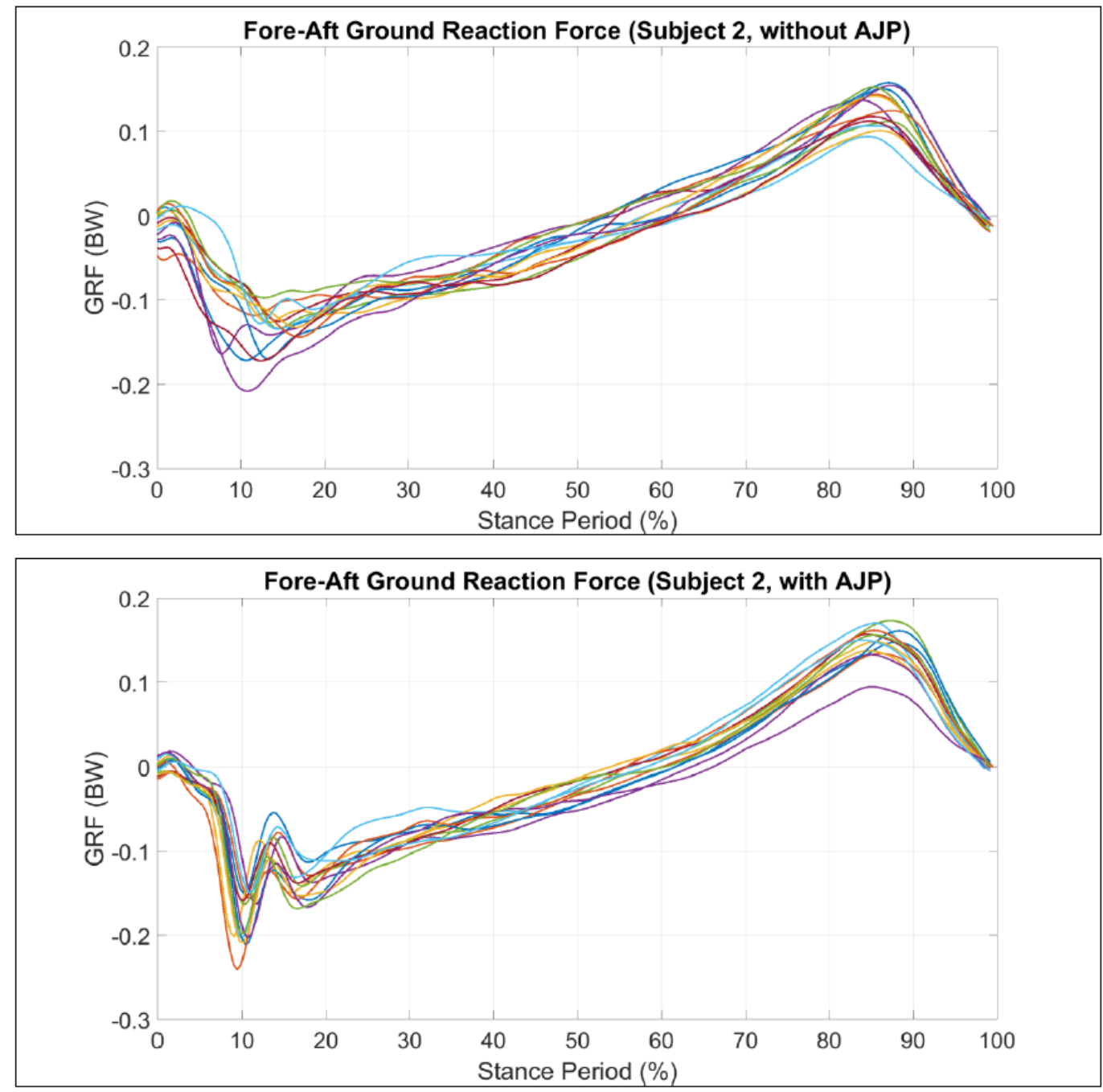

Figure 11.6: Fore-Aft Ground Reaction Force over Period of Stance of Participant 2.

This figure displays the profile of gore-aft GRF against the period of stance of of the CON tests (top) and EXP tests (bottom) of Participant 2. The graphs reflects that the CON vs EXP fore-aft GRF profile is consistent. This is expected, as the statistical analysis of the CON vs EXP temporal-spatial variables showed no significant difference of means. This evidence of controlled GRF via the control of temporal-spatial variables in the experimental methodology thus adds further validity to the outcomes of the analysis of the kinetic and kinematic results. Further, note that due to the removal of outliers identified during the statistical analysis of the data, both the CON and the EXP tests only contain 13 samples. 


\subsection{Comparing the Results to Theoretical Design Aims and Litera- ture}

The AJP was designed to independently replicate two phases of stance, CPF and CDF, and thus for each phase a separate set of target criteria was identified: $-6^{\circ}$ of plantarflexion at $-0.1 \mathrm{Nm} / \mathrm{kg}$ (thus at a stiffness $-0.96 \mathrm{Nm} / \mathrm{kg}$.rad) during CPF; and $14^{\circ}$ of peak dorsiflexion at $1.19 \mathrm{Nm} / \mathrm{rad} . \mathrm{kg}$ (thus at a stiffness $5.24 \mathrm{Nm} / \mathrm{rad} . \mathrm{kg}$ ) during CDF. Yet, in order to accommodate for the sagittal plane flexibility of the baseline AFP, the spring set that responds to dorsiflexion was over-designed by $47 \%$ of this value $(7.69 \mathrm{Nm} / \mathrm{rad} . \mathrm{kg})$. This over-design was expected to be neutralised by any inherent flexibility in the baseline AFP. The spring set that controls plantarflexion during initial ground impact was selected based on the availability of a standard manufactured spring that would theoretically introduce the target value of CPF stiffness $(-0.88 \mathrm{Nm} / \mathrm{rad} . \mathrm{kg} ; 92 \%$ of target value). Thus the expectation from the results of this study is that EXP CDF and CPF stiffness replicates the target criteria of $5.24 \mathrm{Nm} / \mathrm{rad} . \mathrm{kg}$ and $-0.96 \mathrm{Nm} / \mathrm{rad} . \mathrm{kg}$ respectively.

Recall the literature review of Su et al. [2010] in Section 3.5, where the effect that the attachment of a sagittal plane flexion unit (Multiflex Ankle) to a baseline AFP (Seattle Lightfoot) has on TT amputee stance mechanics was analysed. The results of that study are further used here, due to the fact that baseline AFP's used in Su et al. [2010] and this study are classified as SACHDyanmic AFP's [NZALS, 2018], and as such Su et al. [2010] can be used as a comparative study. The work of Gard [2011] is also incorporated into this analysis, although it must be stated here that Gard [2011] is a retrospective analysis of the data collected in Su et al. [2010], and both papers must be considered as an analysis of the same dataset. The phase termination points of the baseline is approximated from the graph generated in Gard [2011] to be $-2^{\circ}$ and $0.18 \mathrm{Nm} / \mathrm{kg}$ for CPF, yielding a linear $-5.16 \mathrm{Nm} / \mathrm{rad} . \mathrm{kg}$ approximation of stiffness, and $10^{\circ}$ and $1.12 \mathrm{Nm} / \mathrm{kg}$ for CDF, yielding a $6.42 \mathrm{Nm} / \mathrm{rad} . \mathrm{kg}$ linear approximation of stiffness. The phase termination points of the baseline with the addition of the flexion unit is approximated at $-4^{\circ}$ and $0.13 \mathrm{Nm} / \mathrm{kg}$ for CPF, yielding a $-1.86 \mathrm{Nm} / \mathrm{rad} . \mathrm{kg}$ approximation of stiffness, and a $15^{\circ}$ and $1.09 \mathrm{Nm} / \mathrm{kg}$ for CDF, yielding a $4.16 \mathrm{Nm} / \mathrm{kg}$.rad linear approximation of stiffness.

Thus, it is evident from the results of Su et al. [2010]; Gard [2011] that in comparison to the able-bodied stance mechanics that Su et al. [2010] collected (see Section 3.5), the baseline unit yields both CPF and CDF stiffness that is significantly greater than that of the ideal (able-bodied) stiffness. Yet, the results indicate that whilst the flexion unit reduces the stiffness of both phases, the CPF stiffness has been under reduced (from $-5.16 \mathrm{Nm} / \mathrm{rad} . \mathrm{kg}$ to $-1.86 \mathrm{Nm} / \mathrm{rad} . \mathrm{kg}$, failing to reproduce $-0.95 \mathrm{Nm} / \mathrm{rad} . \mathrm{kg}$ ) and the CDF stiffness has been over reduced (from $6.42 \mathrm{Nm} / \mathrm{rad} . \mathrm{kg}$ to $4.16 \mathrm{Nm} / \mathrm{rad} . \mathrm{kg}$, as opposed to the able-bodied target of $5.24 \mathrm{Nm} / \mathrm{rad} . \mathrm{kg}$ ).

The results of this study confirm that the baseline AFP's dorsiflexion capacity is characterised as stiff relative to the able bodied CDF stiffness (5.24 Nm/rad.kg [Su et al., 2010]), as evident in the CON CDF stiffness across all 3 participants $(10.99 \mathrm{Nm} / \mathrm{kg} . \mathrm{rad}, 7.38 \mathrm{Nm} / \mathrm{rad} . \mathrm{kg}$, and 7.39 $\mathrm{Nm} / \mathrm{rad} . \mathrm{kg}$ for each participant respectively). Note how the baseline AFP CDF stiffness for par- 
ticipant 2 and 3, who are of very similar demographics, is near identical. Further, these results indicate that the baseline AFP is stiffer in dorsiflexion than the Seattle Lightfoot II. Yet as with the Multiflex Ankle, the addition of the designed AJP to the baseline AFP reduced the stiffness of the apparatus to beyond the target value (4.99 Nm/rad.kg, 3.77 Nm/rad.kg and $4.29 \mathrm{Nm} / \mathrm{rad} . \mathrm{kg}$ for each participant respectively). Recall that the AJP was over designed by a factor of $1.5(47 \%)$ in anticipation of the undefined inherent flexibility of the baseline AFP, and the results would appear to indicate that this over-design should have been to a greater factor. However it is likely that this over-reduction of stiffness is associated with the inherent flexibility of the PP structure, which is a methodology complication that arises when simulating TT amputee gait as opposed to using amputees directly. This complication will be further discussed in Section 11.3. Yet it is encouraging that the baseline + flexion unit in Gard [2011] yields a CDF stiffness (4.16 Nm/rad.kg) that falls into the range of the baseline AFP + AJP, thus validating that the isolated design of the AJP is producing results on par with that of the Multiflex Ankle. However it is disappointing that the AJP prototype used in this iteration of the project has also failed to accurately reproduce the idealised stance mechanics, but due to the modular nature thereof it is believed that by replacing the CDF springs with as stiffer set a more accurate replication of these idealised mechanics can be achieved.

In terms of the CPF phase replication, the target stiffness was identified as $-0.95 \mathrm{Nm} / \mathrm{rad}$. $\mathrm{kg}$. The EXP results yield CPF stiffness values of $-0.11 \mathrm{Nm} / \mathrm{rad} . \mathrm{kg},-0.15 \mathrm{Nm} / \mathrm{rad} . \mathrm{kg}$ and $-0.07 \mathrm{Nm} / \mathrm{rad} . \mathrm{kg}$ for each participant respectively. In contrast, the data of Gard [2011] suggests a CPF stiffness of $1.86 \mathrm{Nm} / \mathrm{rad} . \mathrm{kg}$ with the addition of the flexion unit. Equally concerning is the large discrepancy between the CPF stiffness of the CON tests $(-0.39 \mathrm{Nm} / \mathrm{rad} . \mathrm{kg},-0.51 \mathrm{Nm} / \mathrm{rad} . \mathrm{kg}$ and $-0.74 \mathrm{Nm} / \mathrm{rad} . \mathrm{kg}$ for each participant respectively) and the baseline results of Gard [2011] (-5.16 Nm/kg.rad). A major finding in the review of literature is the inability of basic AFP's to plantarflex at heel contact, yet the results of this study indicate that the CPF stiffness of the baseline AFP with and without the AJP are significantly below expectations. Thus it is obvious that the methodology used here to quantify CPF stiffness has failed. Diagnosis of the error reveals that whilst the methodology used to capture kinematics (angular rotation of the joint) has succeeded, it appears that the error relates to the capture of kinetic data (joint moment). Specifically, the error is generated by inaccurate location of CoP at small magnitudes of GRF, which will be further discussed in Section 11.3. Yet the outcome of this diagnosis is the conclusion that the methodology fails to accurately calculate ankle joint moment at heelstrike and during the transition to footflat. This leaves the outcome of the kinetic analysis of ankle kinetics during CPF inconclusive, and thus the recommendation is that the methodology needs to be adjusted before the impact the AJP has on stance mechanics of CPF can be analysed. The calculation of joint moments via inverse dynamics needs to be reconsidered. However, it is observed in Figure 3.6 that the Multiflex Ankle had a marginal impact on the joint RoM of the baseline AFP during CPF, and fails to replicate the AB joint RoM during this phase. In contrast, the designed AJP is showing significant improvement on the baseline AFP's joint RoM during CPF. As this mechanism was identified in Section 3.2 as being critical to weight acceptance and stability control during early stance, and is undervalued by traditional AFP design, 
improvement in this regard is crucial and joint RoM evidence suggests that the designed AJP is producing better results than that of the Multiflex Ankle.

In spite the errors associated with the experimental methodology, what is clearly evident is that the AJP has successfully achieved the core functional design objectives (FDO's) set out in Section 6.1.1. The device displays the intended allowance of the restrained yet mobile transition from heelstrike to footlfat during CPF as per FDO (1), specifically a significant improvement of the baseline AFP foot joint angle RoM during this phase. There are also strong indications that the AJP is more successful in the replication of this stance mechanism than comparative attempts (i.e. the Single Axis foot). However, due to the measurement methodology limitations, it is not yet possible to definitively compare the CPF performance of the AJP to that of natural stance mechanics nor that of its closest competitor, the Endolite Multiflex Ankle. Also clearly evident is the ability of the AJP to facilitate the control of tibial progression (i.e. CDF), as per FDO (2). The results show that the device is drastically improving the RoM of the ankle joint angle during CDF whilst maintaining a consistent moment across CON and EXP trials, therefore allowing for an extended period of footflat time and thus improving forefoot rollover shapes of the baseline AFP. Yet the CDF stiffness results show that the device has been under specified in this regard, and thus re-specification of the springs is required to improve the replication accuracy of natural stance mechanisms. Finally, there are indications of the release of the elastic energy stored during CDF (FDO (3)), as evident in the steep slope gradient of ankle join moment and the return of the device to neutral position following toe-off. However, this variable requires alternative verification methodologies, such as an analysis of push-off power and metabolic consumption, and qualitative debriefings with participants.

There are concerns relating to the RoM evident in the baseline AFP (i.e. in the CON test) when comparing the results to comparative studies defined in literature. Wagner et al. [1987] documents that baseline AFP's (a SACH foot) display $\pm 6^{\circ}$ of plantarflexion during early stance, yet in this study planatarflexion capacity of $6-9^{\circ}$ is documented. However it must again be noted that due to the inherent flexibility associated with the AIB in the PP/DPP structure and the compressive capacity of the shoe heel, these values cannot be seen as quantification of baseline AFP mechanics. Rather, it must be seen simply as a control test to which the capacity introduced by the AJP can be relatively quantified. This, again, motivates for the recommendation that the baseline AFP + AJP be tested on actual TT amputees.

\subsection{Review of Methodology}

The principle aim of this experimental methodology (described in Chapter 9) is to characterise the impact that the designed AJP has on the stance mechanics of the baseline AFP via the analysis of a simulated TT amputee gait cycle, with and without an active AJP. Yet there are a few self critiques on the methodology used, which are described and discussed in this section. 
The major error with the methodology used in this study is that of the capturing of kinetic data during early stance. As discussed in Section 11.2, a significant error occurred when attempting to calculate ankle joint moment at heelstrike and the transition to footflat, in that the CPF stiffness for EXP tests across participants yielded results that are less than $10 \%$ of the target stiffness. Lower than expected results are also recorded during the CON tests. The diagnosis of this issue revealed that the error lies in the inability of the AMTI force plate to accurately record CoP at early stages of stance. Figure 11.7 displays the sagittal plane position of CoP and the ankle CoR of one focus trial of Participant 2.

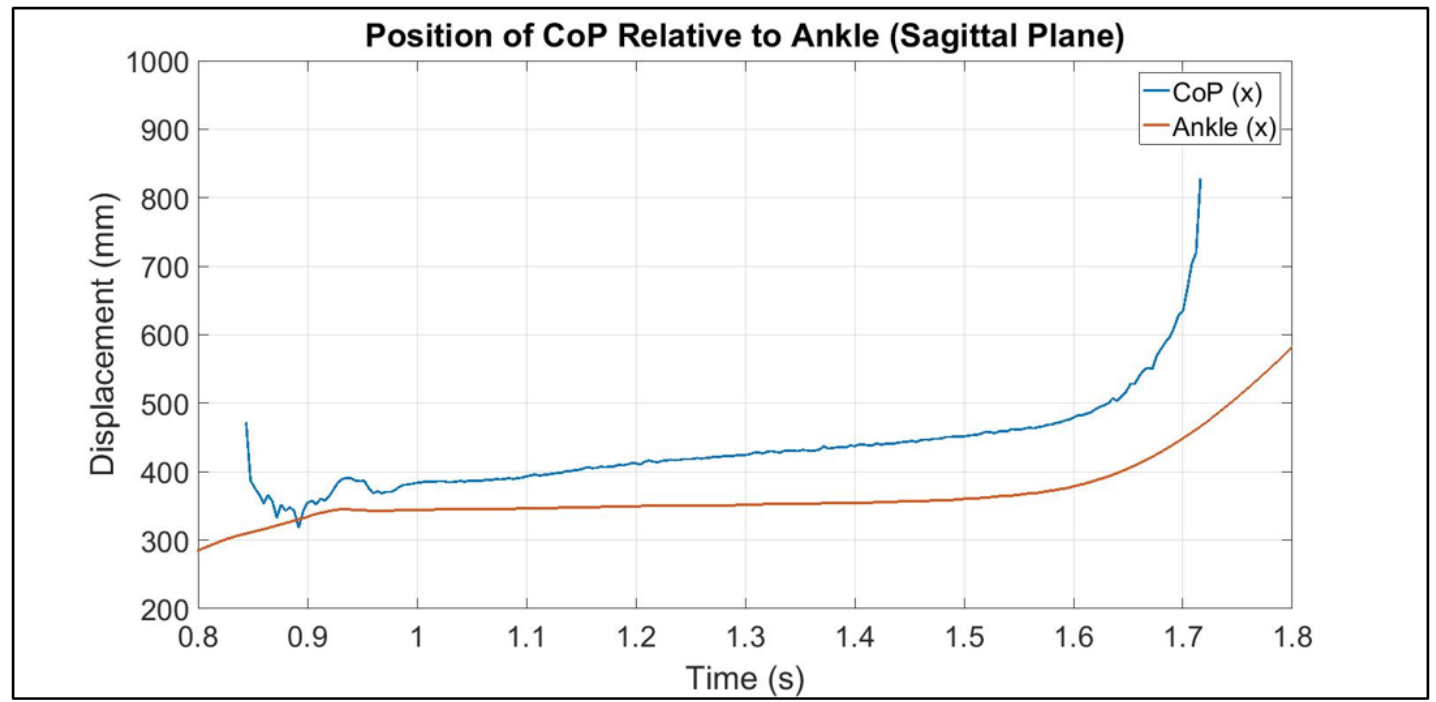

Figure 11.7: Location of CoP Relative to Ankle Position in Sagittal Plane.

This figure graphically displays the position of the CoP of GRF as recorded by the AMTI Force Plate and the position of the ankle joint marker. The data is extracted from the same trial of Participant 2 as that seen in Figure 11.3. Note that the CoP dataset has been intentionally left unfiltered for the purpose of visually describing the erratic readings that are associated with $\operatorname{GRF}(\mathrm{z})$ magnitudes of under $200 \mathrm{~N}(0.24 \mathrm{BW})$. Force plate data reveals that the GRF magnitude is below this threshold before $0.89 \mathrm{~s}$ and after $1.67 \mathrm{~s}$. These time intervals corresponds to the unexpected CoP location data seen during early stance/at heelstrike (where peak joint CoP distance is recorded $\pm 300 \mathrm{~mm}$ anterior of the ankle joint) and during late stance/at toe-off (where peak joint to CoP distance is recorded at $\pm 350 \mathrm{~mm}$ anterior of the ankle). Thus it is concluded that the AMTI FP fails to accurately describe the CoP location for GRF magnitude of under $200 \mathrm{~N}$, and as such fails to allow for the calculation of moment arm lengths for GRF's under this threshold. Due to the fact that during the majority of CPF/early stance the GRF falls under this threshold, it is concluded that the methodology used to determine joint moments (see Equation 9.2) will fail to quantify joint moments during the majority of this phase.

Analysis of ankle joint angle for this focus trial reveals that the foot reached footflat at $\pm 0.9 \mathrm{~s}$, after undergoing $\pm 8^{\circ}$ of plantarflexion. This confirms that CPF took place as intended, which would imply that the GRF reaction to footflat would have created a moment around the ankle joint that forced the foot to plantarflex. In order to create this plantarflexion moment the GRF CoP would need to be located at least in line with the ankle joint sagittal plane location, if not posterior thereof.

Yet Figure 11.7 shows that at the initiation of stance the CoP appears $\pm 300 \mathrm{~mm}$ (approximately 
one foot length) ahead of the ankle joint, and is only posterior to the ankle joint for one datapoint during early stance (at $\pm 0.89 \mathrm{~s}$ ). Thus, the CoP GRF prior to $\pm 0.89 \mathrm{~s}$ is obviously yielding incorrect readings. Referring to Equation 9.2, it can be seen that the moment arm of the GRF is calculated as the difference in CoP and ankle joint location in the sagittal plane. At stance initiation the CoP location of $\operatorname{GRF}(z)$ is expected to be posterior of the ankle position, and would thus create a clockwise moment around the ankle joint that forces the foot to plantarflex. Combined with the clockwise moment induced by the $\operatorname{GRF}(x)$ moment (due to the fact that $\operatorname{Gr}(x)$ is negative during early-mid stance), a net clockwise moment around the ankle joint is thus expected. Yet the the error in recording of CoP at heelstrike results in a negative (opposite) moment arm length, and thus an anti-clockwise GRF(z) moment is calculated that opposes the clockwise induced GRF(x) moment, yielding reduced a 'peak moment' of CPF relative to that which is expected.

Further, it can be seen that CoP stabilises as the BW transfers anterior to the ankle, gradually increasing from $\pm 0 \mathrm{~mm}$ (at $\pm 0.89 \mathrm{~s}$ ) to $\pm 130 \mathrm{~mm}$ anterior of the ankle position, at which point the foot approaches toe-off and GRF(z) dips below $200 \mathrm{~N}$ (at $\pm 1.7 \mathrm{~s}$ ). For a foot of $280 \mathrm{~mm}$ in length (and thus an approximate distance from the ankle joint to the metatarsal-phalangeal joint of \pm 130 $\mathrm{mm}$ ), this seems an appropriate location of CoP. Yet, notice that immediately after $1.7 \mathrm{~s}$ the CoP exponentially spikes exponentially to a peak of $\pm 350 \mathrm{~mm}$ anterior of the ankle. This is a repeat of the CoP error seen in early stance, which leads to the assumption that this error is being generated at GRF magnitudes of less than $200 \mathrm{~N}$ (0.24 BW). During CDF the GRF(z) magnitude can peak at $1 \mathrm{BW}$, and thus is well clear of the minimum magnitude required to accurately record CoP. However, the GRF(z) during CPF peaks at termination of the phase at a magnitude of $0.36 \mathrm{BW}$ (for this participant), and thus the majority of the phase is under the minimum required magnitude to record accurate CoP.

Equation 9.2 and the associated data processing methodology is based on that of Hansen et al. [2004], and the authors of the study similarly found it difficult to quantify joint moment during early stance and noted that the force plate (also AMTI) failed to accurately locate CoP at GRF(z) magnitudes under $120 \mathrm{~N}$. The results of Hansen et al. [2004] indicate a peak joint moment during CPF of approximately $0 \mathrm{Nm} / \mathrm{kg}$, despite plantarflexion of between $5^{\circ}$ and $8^{\circ}$ (at slow and fast walking velocities, respectively), implying that the natural foot/ankle has no stiffness during CPF. This is in contrast to statistically significant linear characterisation of CPF by Palmer [1999] across a range of subjects, who used the inverse dynamics approach.

Thus the conclusion from this error diagnosis is that the methodology of Hansen et al. [2004] is not appropriate when attempting to define the kinetics of the CPF phase i.e. early stance. Therefore, whilst the characterisation of CPF kinematics yielded positive results (i.e. the increase of angle RoM) and all indications are that the AJP improves rollover shape and phase transitions during early stance, the characterisation of stance kinetics for CPF in this study must be declared inconclusive. This is unfortunate, as characterising the stance mechanics of CPF is crucial to the analysis of the impact that the AJP has on baseline AFP mechanics, and it is highly recommended that future analysis of the AJP utilises alternative kinetic analysis methodologies (such as the reexploration of inverse dynamics). 
As discussed earlier, the experimental methodology attempts to produce consistent intra-participant walking velocity variable due to $\mathrm{KK}$ variation that is associated with increased walking velocity Hansen et al. [2004]. Yet normal distribution of the CON and EXP datasets is required in order to analyse this variable via an independent T-test, and statistical analysis of these two datasets for Participant 1 and 3 reveals that none are normally distributed. Ideally walking velocity would be calculated by the division of distance travelled over a number of strides by the time period, assuming that net acceleration has reached zero. However, this methodology attempts to calculate walking velocity from the raw kinetic and kinematic data in post processing i.e. how far the ankle joint marker travelled over the period of the one focus stride, measured from footflat to footflat (the initial and secondary local minimums of ankle joint angle). Further, the Vicon motion capture apparatus has a limited focus range that only allows for the adequate recording of markers (using this custom marker set on the PP/DPP) for a duration of approximately two to three strides. Thus one issue that arises is that walking velocity is calculated based on one stride length of only the focus (right) limb, whereas at minimum walking velocity should be calculated from two stride lengths (focus limb and then contralateral limb, if we are to assume that double stride length/time is consistent). A further flaw in this testing methodology is that it does not necessarily guarantee that net acceleration of zero has been reached, as the participant begins from a standing start half a stride length away from initiating the focus (right) stride (see Section 9.2.4). The combination of these methodology flaws could be the cause of non-normal distribution of walking velocity, and thus the conclusion from this critique is that an alternative method of recording walking velocity must be incorporated.

As stipulated in Section 6.2, the AJP was designed based on stance mechanics derived from an able-bodied population of seven males and seven females, of mean mean demographics 26 years, $1.74 \mathrm{~m}$ in height and $72.3 \mathrm{~kg}$ in mass, walking at a self-selected slow mean velocity of $0.82 \mathrm{~m} / \mathrm{s}$ Su et al. [2010]. Yet the participant population available for this study was limited to three males of ages 21,24 and 29 years, masses of $81.5 \mathrm{~kg}, 85.0 \mathrm{~kg}$ and $85.0 \mathrm{~kg}$, and heights of $1.93 \mathrm{~m}$, $1.83 \mathrm{~m}$ and $1.80 \mathrm{~m}$. Thus the mean mass and height off this participant population sits well above the demographics of the population on which the AJP has been designed, although ankle joint moment has consistently been normalized by mass in literature [Hansen et al., 2004; Su et al., 2010] and by mass and height in other cases [Kerrigan et al., 1998]. Another potential concern is the gender bias of this methodologies participant population, yet Kerrigan et al. [1998] found mostly no significant differences in inter-gender gait kinetics and kinematics despite finding significant differences in stride length and walking velocity. A further concern relates to the height of Participant 1 , who is $10 \mathrm{~cm}$ and $13 \mathrm{~cm}$ taller than Participant 2 and 3 respectively, as there is an expectation that increased height would result in increase stride length and thus increased plantarflexion at heelstrike/dorsiflexion at toe-off. Yet whilst inter-subject statistical analysis must be conducted in order to conclude any analysis on these inter-variable relationships, from the results it is evident that the 95\% Cl of Participant 1's CON and EXP mean stride lengths overlap with those of Participant 2's. Further, the EXP walking velocity for both Participants 1 and 2 overlap (although the CON walking velocity of Participant 1 is slower than that of Participant 2). This indicates that, despite the height 
difference, both participants produce similar walking velocities and stride lengths when using the AJP, and thus the height variance is not destabilising the trend analysis. An interesting result from the analysis is that the $95 \% \mathrm{Cl}$ of walking velocity and stride length of Participant 2 and 3 , who have near identical height and weight demographics, do not overlap. Thus the conclusion is that the relationship between inter-subject height and weight variation and the controlled experimental TS variables (walking velocity and stride length) is not yet a concern, although this is pending a broader inter-participant statistical analysis on these inter-variable relationships. 


\section{Chapter 12}

\section{Conclusions}

The a-priori research conducted in this project motivates why the restoration of ankle joint mechanisms for the purpose of improving stance mechanics will have a positive impact on TT amputee walking gait cycles. As such, AJP that is attachable to the proximal head of baseline AFP's was designed with the intention of replicating the functional ankle mechanisms evident in natural stance mechanics (quantified as objectives). The prototype designed for testing in this study has additionally been proven to be low-cost and low-maintenance, thus fulfilling the aim of producing a device that can sustainably serve the low-income amputee population in developing countries. The experimental aim of this project was to quantify the impact that the designed AJP has on the stance mechanics of a baseline AFP, which in this particular case is a SACH-Dynamic foot (the Otto Bock 1D10). In order to do so, the TT amputee gait cycle was simulated via the use of a pseudoprosthesis system and three able-bodied participants. This methodology allowed for quantification of the impact that the designed AJP has on the stance mechanics of the baseline AFP based on predefined kinetic and kinematic metrics, and negates the need to involve amputees.

The results are positive, indicating that in most areas the AJP statistically improves the stance mechanics of the baseline AFP. In attempting to replicate CDF, the AJP improves forefoot rollover shapes by providing a stable joint moment and a 80\%-132\% $(p<0.001)$ increase in joint RoM, allowing for a prolonged period of footflat during forward tibial progression. The net result is a $42-56 \%$ decrease in ankle CDF stiffness ( $p<0.001$ for all but one participant). Yet indications are that this is an over reduction in stiffness, and as such the recommendation is the re-selection of the pseudo-plantarflexor springs. In attempting to replicate the natural CPF weight acceptance mechanism during early stance the AJP improved plantarflexion mobility by $22 \%-77 \%(p<0.001)$. Visual analysis of ankle flexion revealed that participants initiated stance with a heel strike when the AJP was activated, yet incorporated a flatter foot impact when relying on the baseline AFP, indicating that the AJP provides an improved transition from heelstrike to the stable position of footflat. However equipment and methodology errors results in the inability to accurately define joint kinetics for GRF's below $200 \mathrm{~N}$, and thus the joint moments prevalent during early stance were unverifiable. Thus despite evidence of a $50 \%+$ reduction in ankle stiffness during this phase, it is officially stated as unquantified and in need of re-analysis using an altered methodology. 
These results are comparable to that of the Multiflex Ankle documented in [Su et al., 2010; Gard, 2011], although a comparative experimental study is recommended. Common trends relate to CDF mechanics, yet analysis of joint RoM during CPF indicates that the designed AJP is providing better mobility in this regard. This phase of stance was specifically identified as being of critical design importance due to its facilitation of stability control during early stance, despite being largely ignored in traditional AFP design.

Overall, the conclusions are that the AJP has a positive impact on the stance mechanics of a baseline AFP. The results show that the device improves rollover shapes, eases phase transitions and appropriately facilitates forward progression. In combination with the low cost price of the prototype (R595/50 USD), its ease of assembly and modular design, the designed AJP is thus a potentially preferable option for low-income amputees in developing countries. Finally, the device has provided significant evidence of functional and mechanical reliability, and therefore testing of the device can progress to a clinical study involving amputee participants. In anticipation thereof, the major recommendations that arise relate to the design of a mass and height based spring selection guideline, and the use of an inverse-dynamics data processing methodology to better quantify stance kinetics during early stance. 


\section{References}

Aerts, P., Ker, R. F., De Clercq, D., Ilsley, D. W., and Alexander, R. M. (1995). The mechanical properties of the human heel pad: A paradox resolved. Journal of Biomechanics, 28(11):12991308.

Alexander and McNeill, R. (1984). Walking and Running. American Scientist, 72(4):348-354.

Bakker, K., Houtum, W. H. V., and Riley, P. C. (2005). 2005 : The International Diabetes Federation Focuses on the Diabetic Foot. Technical report.

Barr, a. E., Siegel, K. L., Danoff, J. V., McGarvey, C. L., Tomasko, a., Sable, I., and Stanhope, S. J. (1992). Biomechanical comparison of the energy-storing capabilities of SACH and Carbon Copy II prosthetic feet during the stance phase of gait in a person with below-knee amputation. Physical therapy, 72(5):344-354.

Boulton, A. J. M., Vileikyte, L., Ragnarson-tennvall, G., and Apelqvist, J. (2004). The global burden of diabetic foot disease. Diabetes Care, 27:1047-53.

Breakey, J. (1976). Gait of Unilateral Below-Knee Amputees. Orthotics and Prosthetics, 30(3):1724.

Brockett, C. L. and Chapman, G. J. (2016). Biomechanics of the ankle. Orthopaedics and Trauma, 30(3):232-238.

Budynas, R. and Nisbett, J. (2011). Shigley’s Mechanical Engineering Design. New York, McGrawHill.

Collins, S. H. and Kuo, A. D. (2010). Recycling energy to restore impaired ankle function during human walking. PLOS ONE, 5(2).

Dillingham, T. R., Pezzin, L. E., and Mackenzie, E. J. (1996). Limb Amputation and Limb Deficiency : Epidemiology and Recent Trends in the United States. Southern Medical Journal, 95(8):875884.

Dillingham, T. R., Pezzin, L. E., Mackenzie, E. J., Tr, A. D., Le, P., and Ej, M. (2002). Racial Differences in the Incidence of Limb Loss Secondary to Peripheral Vascular Disease : A PopulationBased Study. Archives of Physical Medicine and Rehabilitation, 83(September):1252-1257. 
Ellis, E. (2016). 700 Amputations at Hospital. Accessed at: http://www.heraldlive.co.za/news/topnews/2016/06/27/700-amputations-hospital/. Last accessed on: 24/01/18.

Endolite (2017). Multiflex Ankle Clamp -Instructions for Use. Accessed at: $\quad$ http://www.endolite.com/catalogue/ankles-adapters/ankles/multiflex-standard30mm/ifu/938224 Iss3.pdf. Last accessed on 2018-01-19.

Eng, J. J. and Winter, D. A. (1995). Kinetic analysis of the lower limbs during walking: What information can be gained from a three-dimensional model? Journal of Biomechanics, 28(6):753758.

Gailey, R., Allen, K., Castles, J., Kucharik, J., and Roeder, M. (2008). Review of secondary physical conditions associated with lower-limb amputation and long-term prosthesis use. Journal of Rehabilitation Research and Development, 45(1):15-29.

Gard, S. A. (2011). Effect of prosthetic ankle units on roll-over shape characteristics during walking in persons with bilateral transtibial amputations. Journal of Rehabilitation Research and Development, 48(9):1037-1048.

Gates, D. H., Dingwell, J. B., Scott, S. J., Sinitski, E. H., and Wilken, J. M. (2012). Gait characteristics of individuals with transtibial amputations walking on a destabilizing rock surface. Gait and Posture, 36(1):33-39.

Gates, D. H., Lelas, J., Della Croce, U., Herr, H., and Bonato, P. (2004). Characterization of ankle function during stair ambulation. Conference proceedings : ... Annual International Conference of the IEEE Engineering in Medicine and Biology Society. IEEE Engineering in Medicine and Biology Society. Conference, 6:4248-4251.

Giles, E. and Vallandigham, P. H. (1991). Height estimation from foot and shoeprint length. Journal of Forensic Sciences, 36(4):1134-1151.

Godlwana, L., Nadasan, T., and Pucktree, T. (2008). Global Trends in Incidence of Lower Limb Amputation :. SA Journal of Physiotherapy, 64(Henry 1993):1-5.

Hansen, a. H., Childress, D. S., and Knox, E. H. (2000). Prosthetic foot roll-over shapes with implications for alignment of trans-tibial prostheses. Prosthetics and Orthotics International, 24(3):205-215.

Hansen, A. H., Childress, D. S., Miff, S. C., Gard, S. A., and Mesplay, K. P. (2004). The human ankle during walking: Implications for design of biomimetic ankle prostheses. Journal of Biomechanics, 37(10):1467-1474.

Henry, J. (1993). A decriptive survey of lower-limb amputees admitted at Tygerberg hospital (19851987): Theses (unpublished). University of Stellenbosch.

International Diabetes Federation (2011a). Global Diabetes Atlas (5th edition). Technical report. 
International Diabetes Federation (2011b). Global Diabetes Atlas (5th edition). Technical report.

ISO (2010). Tools for pressing Compression springs with rectangular section Housing dimensions and colour coding. ISO 10243:2010.

ISO (2015). Quality Management Systems. ISO 9001:2015.

Kadaba, M. P., Ramakrishnan, H. K., and Wootten, M. E. (1990). Measurement of Lower Extremity Kinematics During Level Walking. Journal of Orthopaedic Research, pages 383-392.

Kepple, T. M., Siegel, K. L., and Stanhope, S. J. (1997). Relative contributions of the lower extremity joint moments to forward progression and suport during gait. Gait \& Posture, 6:1-8.

Kerrigan, D. C., Todd, M. K., and Della Croce, U. (1998). Gender differences in joint biomechanics during walking: normative study in young adults. American Journal of Physical Medicine \& Rehabilitation, 77(1):2-7.

Kubheka, B. (1993). An evaluation of community based rehabilitative care given to amputated patients living in the suburban areas north of Durban: Theses (unpublished). University of Kwazulu-Natal.

Kuo, A. D. (2001). Energetics of Actively Powered Locomotion Using the Simplest Walking Model. Journal of Biomechanical Engineering, 124(1):113.

Lehmann, J. F., Price, R., Boswell-bessette, S., Dralle, A., and Quest, K. (1993). Comprehensive Analysis of Dynamic Elastic Response Feet : Seattle Ankle / Lite Foot Versus SACH Foot. Archive of Physical Medicine Rehabilitation, 74(August).

Levene, H. (1960). Robust tests for equality of variance. In Contributions to Probability and Statistics (I. Olkin, ed.) 278292. Stanford Univ. Press, Palo Alto, CA.

Loram, I. D. and Lakie, M. (2002). Direct measurement of human ankle stiffness during quiet standing : the intrinsic mechanical stiffness is insufficient for stability. Journal of Physiology, 545(3):1041-1053.

Magnusson, L., Ahlström, G., Ramstrand, N., and Fransson, E. I. (2013). Malawian prosthetic and orthotic users' mobility and satisfaction with their lower limb assistive device. Journal of Rehabilitation Medicine, 45(4):385-391.

Magnusson, L., Ramstrand, N., Fransson, E. I., and Ahlström, G. (2014). Mobility and satisfaction with lower-limb prostheses and orthoses among users in Siera Leone: A cross-sectional study. Journal of Rehabilitation Medicine, 46(5):438-446.

Neptune, R. R., Kautz, S. A., and Zajac, F. E. (2001). Contributions of the individual ankle plantar flexors to support, forward progression and swing initiation during walking. Journal of Biomechanics, 34(11):1387-1398. 
NZALS (2018). Feet and Ankles. Accessed at: http://www.nzals.co.nz/products/categories/feetand-ankles. Last accessed on 2018-01-19.

Ossur (2018). Flex-Foot $\AA$ Assure. Accessed at: https://www.ossur.com/prostheticsolutions/products/balance-solutions/flex-foot-assure. Last accessed on: 2018-01-19.

Palmer, M. L. (1999). Sagittal Plane Charactrization of Normal Human Ankle Function Across a Range of Walking Gait Speeds. Rehabilitation, pages 1-74.

Perry, J., Boyd, L. A., Rao, S. S., and Mulroy, S. J. (1997). Prosthetic weight acceptance mechanics in transtibial amputees wearing the Single Axis, Seattle Lite, and Flex Foot. IEEE Transactions on Rehabilitation Engineering, 5(4):283-289.

Powers, C., Torburn, L., Perry, J., and Ayyappa, E. (1994). Influence of Prosthetic Foot Design on Sound Limb Loading in Adults With Unilateral Below-Knee Amputations. Archives of Physical Medicine and Rehabilitation, 75(July):825-829.

Schmalz, T., Blumentritt, S., and Jarasch, R. (2002). Energy expenditure and biomechanical characteristics of lower limb amputee gait:. Gait \& Posture, 16(3):255-263.

Shapiro, A. S. S. and Wilk, M. B. (1965). An Analysis of Variance Test for Normality. Biometrika, 52(3):591-611.

Su, P.-F., Gard, S. a., Lipschutz, R. D., and Kuiken, T. a. (2010). The effects of increased prosthetic ankle motions on the gait of persons with bilateral transtibial amputations. American journal of physical medicine \& rehabilitation / Association of Academic Physiatrists, 89(1):34-47.

Torburn, L., Perry, J., Ayyappa, E., and Shanfield, S. L. (1990). Below-knee amputee gait with dynamic elastic response prosthetic feet : A pilot study. Journal of Rehabilitation Research and Development, 27(4):369-384.

Torburn, L., Powers, C. M., Guiterrez, R., and Perry, J. (1995). Energy expenditure during ambulation in dysvascular and traumatic below-knee amputees: a comparison of five prosthetic feet. Journal of Rehabilitation Research and Development, 32(2):111-119.

Vanderpool, M. T., Collins, S. H., and Kuo, A. D. (2008). Ankle fixation need not increase the energetic cost of human walking. Gait and Posture, 28(3):427-433.

Wagner, J., Sienko, S., Supan, T., and Barth, D. (1987). Motion analysis of SACH vs. Flex-Foot in moderately active below-knee amputees. Clinical Prosthetics and Orthotics, 11(1):55-62.

Waters, R. L. and Mulroy, S. (1999). The energy expenditure of normal and pathological gait. Gait and Posture, 9:207-231.

Wells, R. (1981). The projection of the ground reaction force as a predictor of internal joint moments. Bulletin of Prosthetic Researchs, 18(1):15-19. 
Wild, S., Roglic, G., Green, A., Sicree, R., and Hilary, K. (2004). Global prevalence of diabetes: estimates for the year 2000 and projections for 2030. Diabetes Care, 27(5).

WillowWood (2017). Single Axis Foot. Accessed at: https://www.willowwoodco.com/productsservices/feet/low-activity/single-axis-foot/. Last accessed on 2018-01-19.

Winter, D. A., Patla, A. E., Ishac, M., and Gage, W. H. (2003). Motor mechanisms of balance during quiet standing. Journal of Electromyography and Kinesiology, 13:49-56.

Winter, D. A., Patla, A. E., Rietdyk, S., and Ishac, M. G. (2001). Ankle muscle stiffness in the control of balance during quiet standing. J Neurophysiol, 85(6):2630-2633.

Wirta, R. W., Mason, R., Calvo, K., and Golbranson, F. L. (1991). Effect on gait using various prosthetic ankle-foot devices. Journal of Rehabilitation Research and Development, 28(2):1324.

Wu, G., Siegler, S., Allard, P., Kirtley, C., Leardini, A., Rosenbaum, D., Whittle, M., D'Lima, D. D., Cristofolini, L., Witte, H., Schmid, O., and Stokes, I. (2002). ISB recommendation on definitions of joint coordinate system of various joints for the reporting of human joint motionpart I: ankle, hip, and spine. Journal of Biomechanics, 35(4):543-548.

Zajac, F. E., Neptune, R. R., and Kautz, S. A. (2003). Biomechanics and muscle coordination of human walking: Part II: Lessons from dynamical simulations and clinical implications. Gait and Posture, 17(1):1-17. 


\section{Appendices}


Appendix A

AJP Theoretical Stiffness Calculations 
This Appendix serves to illustrate how the force output of the spring sets are calculated. As an example, the methodology for calculating the AJP stiffness under dorsiflexion (i.e. when the pseudoplantarflexors are activated) will be explained.

Figuer A1 displays the internal arrangement of the AJP housing and the location of the spring sets relative to the centre of rotation (axle) in the sagittal plane.

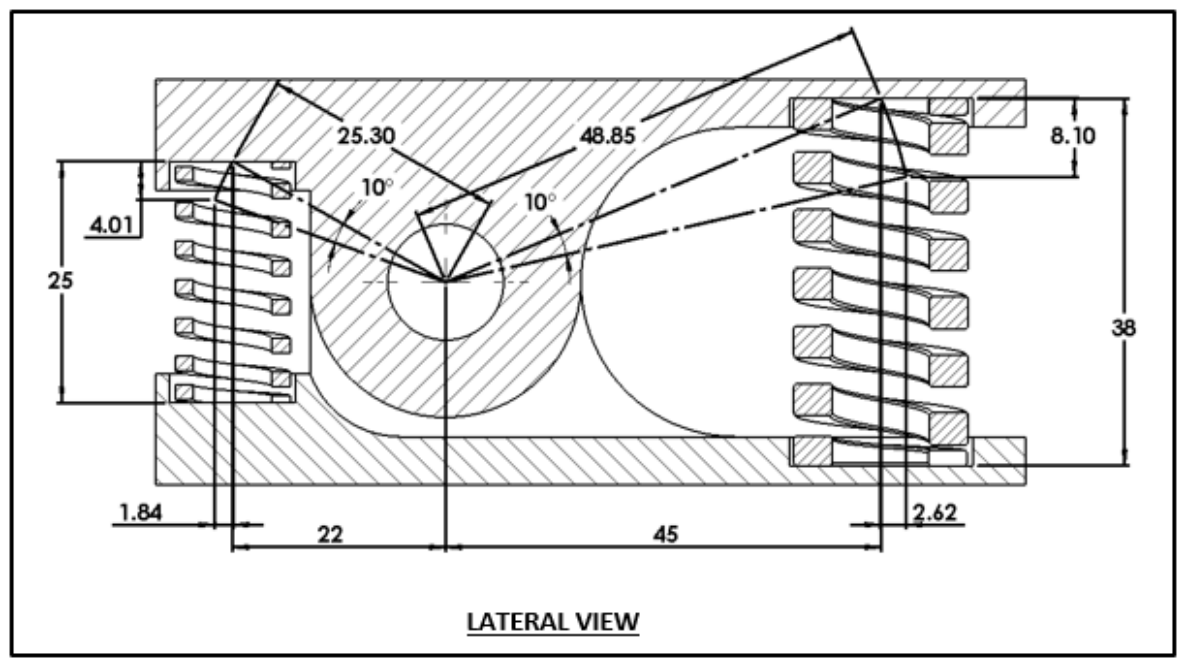

Figure A1: Cross-section of AJP housing.

The relevant variable is thus:

Moment Arm at Neutral (i.e.no compression) $=45 \mathrm{~mm}$

As displayed in Table 7.1, the pseudo-plantarflexor spring set selected consists of $x 2$ springs of:

Spring Free Length $=38 \mathrm{~mm}$

Spring Stiffness $=129 \mathrm{~N} / \mathrm{mm}$

Thus the effective radius from the CoR to the centre of the spring seats is calculated as follows:

$$
\begin{aligned}
\text { Effective Radius }(\text { from CoR }) & =\sqrt{\text { Moment Arm }(\text { at neutral })^{2}+\left(\frac{\text { Free Length }}{2}\right)^{2}} \\
& =48.85 \mathrm{~mm}
\end{aligned}
$$

Thus the angle created between the opposing spring seats, relative to the CoR, at neutral is calculated as follows:

Angle $($ at neutral $)=2 x \tan ^{-1}\left(\frac{\frac{\text { Free Length }}{2}}{\text { Moment Arm (at Neutral })}\right)$ 
Therefore, the angle created when the AJP is under $13^{\circ}$ of dorsiflexion is:

Angle (at maximum dorsiflexion $)=45.78^{\circ}-13^{\circ}$

$$
=32.78^{\circ}
$$

Meanwhile, the maximum dorsiflexion the moment arm has increased to:

Moment Arm (at maximum dorsiflexion)

$$
\begin{aligned}
& =\text { Effective Radius } x \cos \left(\frac{\text { Angle (at maximum dorsiflexion) }}{2}\right) \\
& =46.86 \mathrm{~mm}
\end{aligned}
$$

Therefore, the net compression of each pseudo-plantarflexor spring at maximum dorsiflexion (assuming straight line compression) is:

Spring Compression (at maximum dorsiflexion)

$$
\begin{aligned}
& =2 x \text { Effective Radius from CoR } x\left[\sin \left(\frac{\text { Angle (at neutral) }}{2}\right)\right. \\
& \left.-\sin \left(\frac{\text { Angle }(\text { at maximum dorsiflexion })}{2}\right)\right] \\
& =10.43 \mathrm{~mm}
\end{aligned}
$$

Thus the total spring force response is:

$$
\begin{aligned}
\text { Spring Force Response } & =\text { Number of Springs } x \text { Spring Compression } x \text { Spring Stiffness } \\
& =2961.60 \mathrm{~N}
\end{aligned}
$$

And the moment response is therefore:

$$
\begin{aligned}
& \text { AJP Moment (at maximum dorsiflexion) } \\
& \qquad \begin{array}{l}
=\text { Spring Force Response } x \text { Moment Arm (at maximum dorsiflexion) } \\
=126.13 \mathrm{Nm}
\end{array}
\end{aligned}
$$

Thus a $72.7 \mathrm{~kg}$ participant will yield a moment response of $1.75 \mathrm{Nm} / \mathrm{rad}$.

Assuming a linear relationship, the dorsiflexion stiffness of the AJP can thus be calculated as:

$$
\begin{aligned}
\text { AJP Drosiflexion Stiffness } & =\frac{\text { AJP Moment }(\text { at maximum dorsiflexion })}{\text { Angle }(\text { at maximum dorsiflexion })} \\
& =7.69 \frac{\mathrm{Nm}}{\mathrm{rad} . \mathrm{kg}}
\end{aligned}
$$


Appendix B

Axle Strength Calculations 
This appendix describes the methodology used to analyse the axle under peak dorsiflexion load, in accordance with the free body diagrams displayed Sectio 8.3.

The axle strenght calculations were done in accoradnce with Budynas et al. [2011], whereby the axle was approximated as a beam with simple supports, on which a uniform load is applied. The relevant extract from the textbook is displayed in Figure B2:

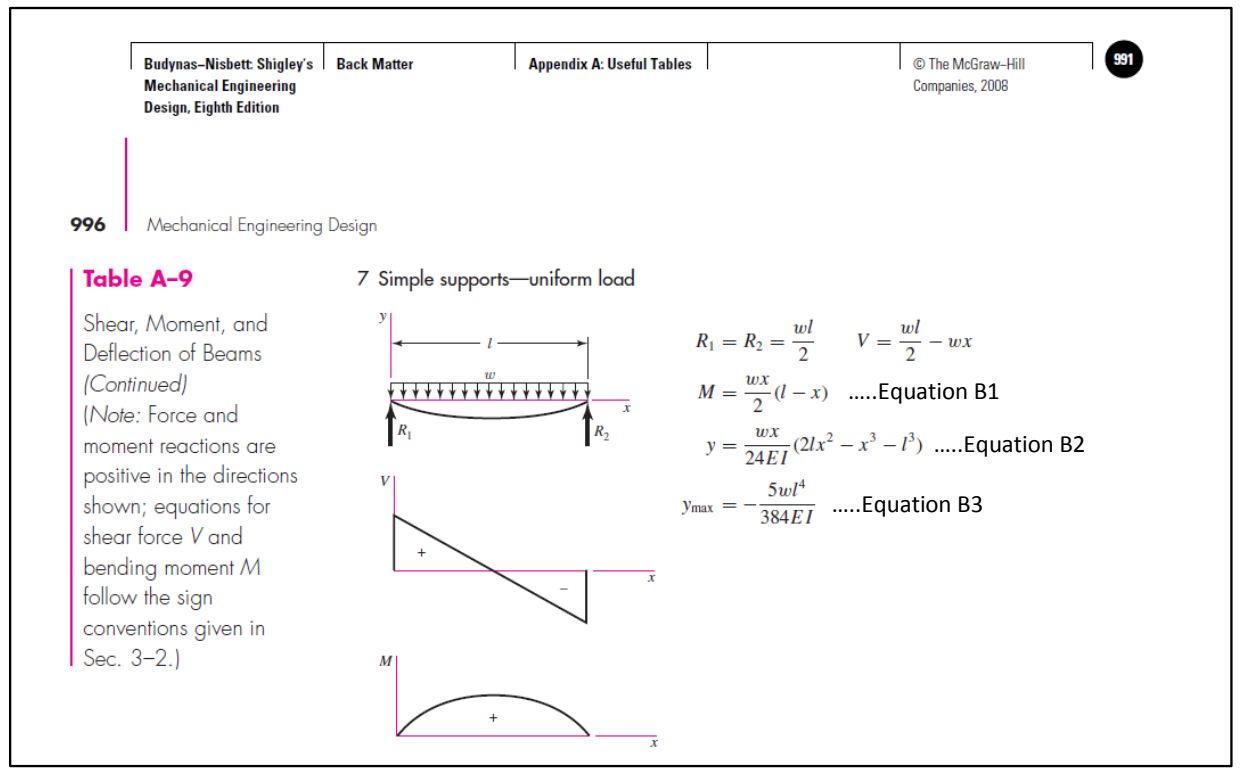

Figure B1: Extract from Budynas et al. [2011]

Where ' $w$ ' is the applied evenly distributed load; ' $L$ ' is the length between supports; ' $x$ ' is measured from ' $R 1$ ' towards ' $R 2$ '; ' $I$ ' is the area moment of inertia; ' $E$ ' is the elastic modulus.

The adaption of the methodology to the axle (a shaft of $12 \mathrm{~mm}$ diameter) is displayed in Figure B2:

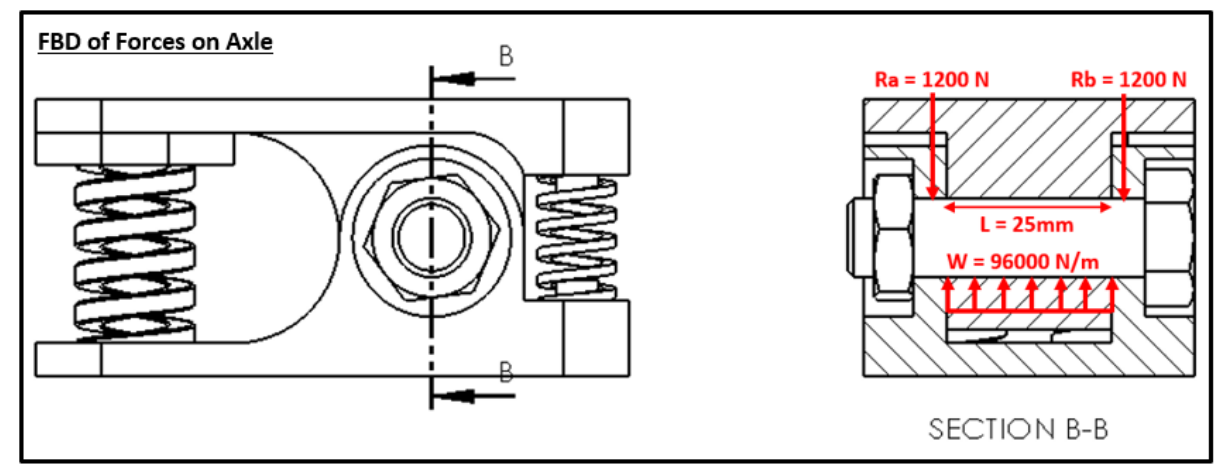

Figure B2: Free Body Diagram of Axle under Load 
Due to the symmetry of the force load, the maximum deflection of the axle under this load is calculated in accordance with the Equation B3 of Figure B1:

$$
\begin{aligned}
y_{\max } & =\frac{5 *\left(96000 \frac{\mathrm{N}}{\mathrm{m}}\right) *(0.025 \mathrm{~m})^{2}}{384 *\left(2 * 10^{11} \mathrm{~Pa}\right) *\left(\pi *\left(\frac{(0.012 \mathrm{~m})^{4}}{64}\right)\right.} \\
& =0.0024 \mathrm{~mm}
\end{aligned}
$$

Equation B1 of Figure B1 is used to calculate the bending moment along the axle (displayed in Figure 8.6) whilst Equation B2 is used to calculate the deflection along the shaft (displayed in Figure 8.6).

For the axle described in Figure B2, the maximum bending stress is found to be $7.50 \mathrm{MPa}$.

The bending stress along the shaft is calculated by the following equation:

Bending Stress $=\frac{M * y}{I}$

Where ' $y$ ' is the vertical distance from the neutral axis (i.e. the centre of the shaft cross sectional area); ' $M$ ' is the bending moment, calculated in accordance with Equation B1; and ' $I$ ' is the area moment of inertia.

Thus the bending stress along the shaft is calculated as:

Bending Stress $(\mathrm{MPa})=\frac{\text { Bending Moment }\left(\text { at ' } x^{\prime} \text { 'along the shaft }\right) * \frac{\text { Shaft Diameter }}{2}}{\pi * \frac{\text { Shaft Diameter }}{4}}$

The material used for the axle is Galvanised Steel, which has a yield stress of $203 \mathrm{MPa}$. Thus the maximum bending stress on the shaft needs to be less than $203 \mathrm{MPa}$. For a shaft of $12 \mathrm{~mm}$, the maximum bending stress is $44.2 \mathrm{MPa}$, thus resulting in a safety factor of 4.59 . 


\section{Appendix C}

Spring Catalogue Sample (ISO 10243) 


\section{DIE SPRINGS}

ISO 10243
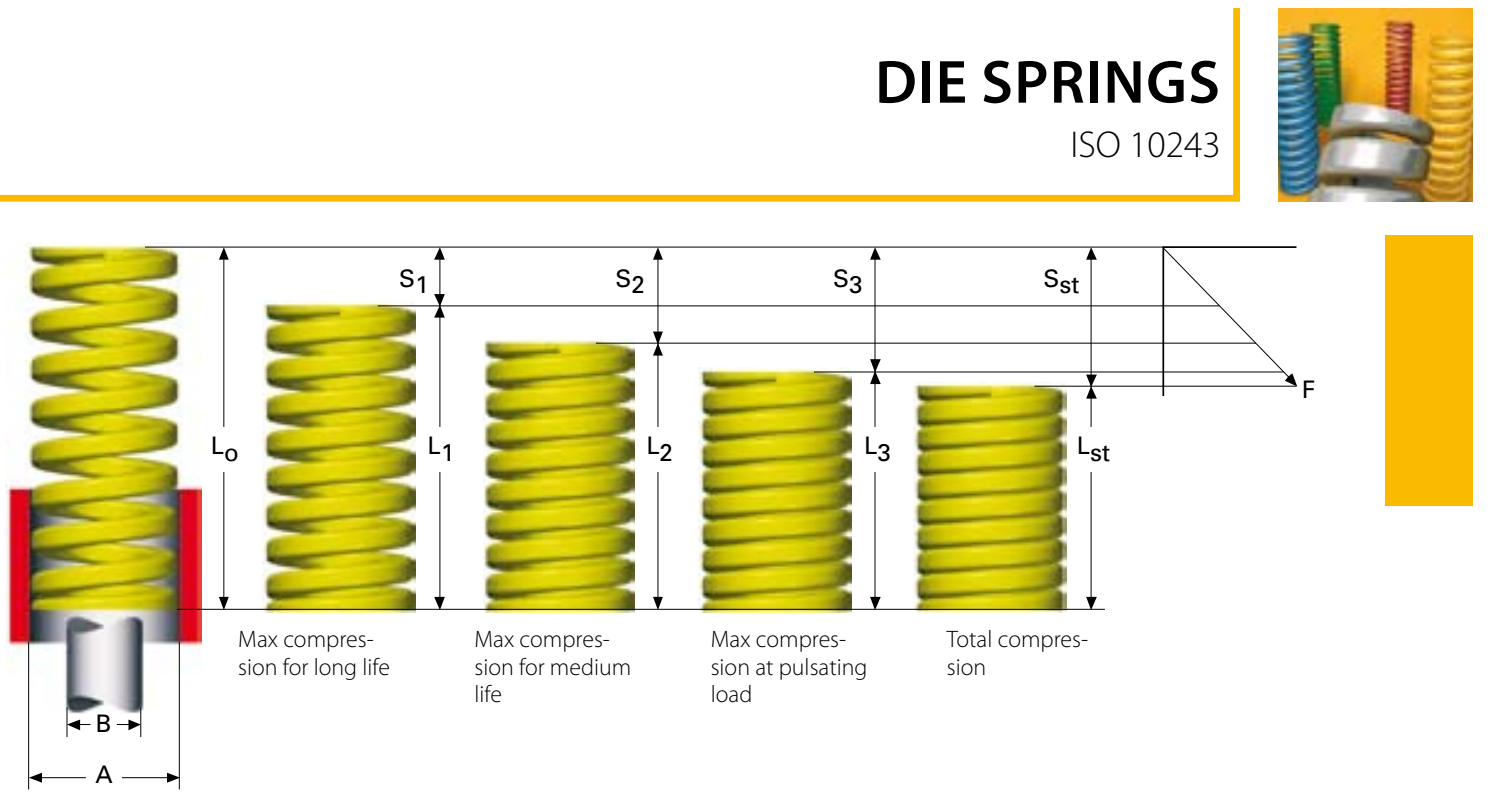

Die springs from rectangular wire are a series of extra high quality compression springs for use in press tools, valves, couplings, fixtures and other applications where high loads are required in a confined space. For each dimension, there are 4-5 different force classes to choose from.

In order to achieve the best characteristics and lifecycle, we follow a well-developed production process.

- A special alloy has been developed, giving improved heat resistance and less relaxation. The closest comparable material is EN 1008951 CrV4.

- Extremely low surface decarburisation free from surface defects.

- The trapezoid profile allows a shorter solid length, thereby increasing deflection capacity.

- A higher permitted level of stress affords a considerably longer lifespan.

- Close tolerances allow for precise fitting in existing space.

\section{How to select a spring}

Compare the available space for the spring to the hole and rod diameter and unloaded length as shown in the catalogue. Then select the suitable force class; 1, 2, 3, 4 or 5 , which is identified by the colour coding system on the next page. The maximum spring deflection stated in the catalogue $\left(S_{3}\right)$ should never be exceeded, as this will shorten the life of the spring. In addition, the spring should always have a certain amount of pre-tension.
All dimensions are in $\mathrm{mm}$

A = Hole diameter

B = Shaft diameter

$\mathrm{L}_{\mathrm{O}} \quad=$ Unloaded length

c $=$ Rate

$\mathrm{s}_{1}=$ Spring deflection at $\mathrm{F}_{1}$

$\mathrm{F}_{\mathrm{n}}=$ Spring force in Newtons at $\mathrm{s}_{1}$

$\mathrm{s}_{2}=$ Spring deflection at $\mathrm{F}_{2}$

$\mathrm{F}_{\mathrm{n}}=$ Spring force in Newtons at $\mathrm{s}_{2}$

$s_{3}=$ Spring deflection at $F_{3}$

$\mathrm{F}_{\mathrm{n}}=$ Spring force in Newtons at $\mathrm{s}_{3}$

$\mathrm{s}_{\mathrm{St}}=$ Spring deflection to solid length

Material: EN 10089 51CrV4

Finish: Epoxy varnish

Tolerances: SS2384, see page 220 for more information.

Max. working temperature: $200^{\circ} \mathrm{C}$

$1 \mathrm{kp}=9.80665$ Newtons, 1 Newton $=0.10197 \mathrm{kp}$ 


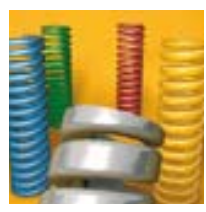

\section{DIE SPRINGS}

ISO 10243

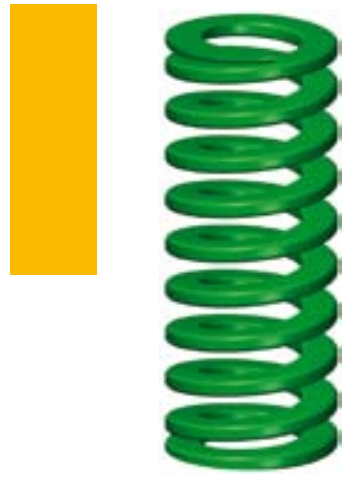

1. Low load

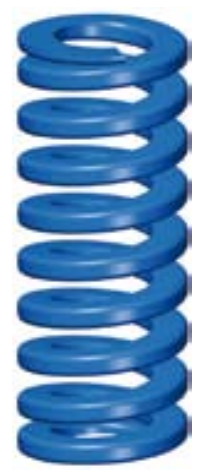

2. Medium load

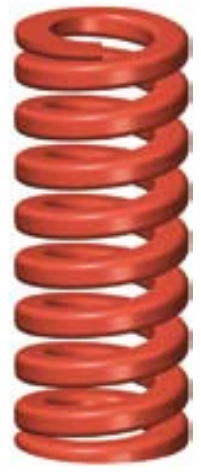

3. High load

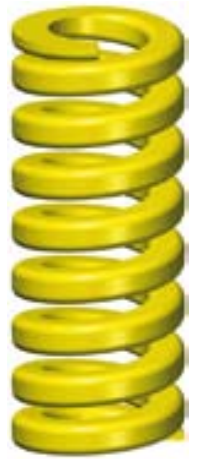

4. Very high load

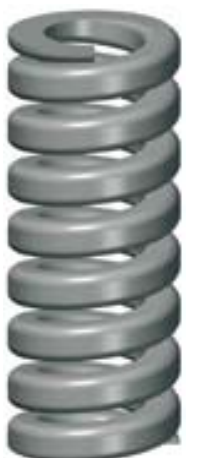

5. Ultrahigh load See separate table on page 69

\begin{tabular}{|c|c|c|c|c|c|c|c|c|c|c|c|c|c|}
\hline $\begin{array}{l}\text { Force } \\
\text { class }\end{array}$ & A & B & $\mathrm{L}_{\mathrm{o}}$ & c & $\mathbf{F}_{1}$ & $s_{1}$ & $\mathbf{F}_{2}$ & $s_{2}$ & $\mathbf{F}_{\mathbf{3}}$ & $s_{3}$ & $\mathbf{F}_{\text {st }}$ & $s_{s t}$ & Cat. no \\
\hline 1 & 10 & 5 & 25 & 10 & 63 & 6,3 & 75 & 7,5 & 100 & 10 & 135 & 13,5 & 5092 \\
\hline 1 & 10 & 5 & 32 & 8,5 & 68 & 8 & 82 & 9,6 & 109 & 12,8 & 149 & 17,5 & 5093 \\
\hline 1 & 10 & 5 & 38 & 6,8 & 65 & 9,5 & 78 & 11,4 & 103 & 15,2 & 141 & 20,8 & 5094 \\
\hline 1 & 10 & 5 & 44 & 6 & 66 & 11 & 79 & 13,2 & 106 & 17,6 & 143 & 23,9 & 5095 \\
\hline 1 & 10 & 5 & 51 & 5 & 64 & 12,8 & 77 & 15,3 & 102 & 20,4 & 145 & 28,9 & 5096 \\
\hline 1 & 10 & 5 & 64 & 4,3 & 69 & 16,0 & 83 & 19,2 & 110 & 25,6 & 155 & 36,1 & 5097 \\
\hline 1 & 10 & 5 & 76 & 3,2 & 61 & 19,0 & 73 & 22,8 & 97 & 30,4 & 138 & 43,2 & 5098 \\
\hline 1 & 10 & 5 & 305 & 1,1 & 84 & 76,3 & 101 & 91,5 & 134 & 122 & 197 & 178,7 & 5099 \\
\hline 2 & 10 & 5 & 25 & 16 & 101 & 6,3 & 120 & 7,5 & 150 & 9,4 & 163 & 10,2 & 5188 \\
\hline 2 & 10 & 5 & 32 & 13 & 104 & 8 & 125 & 9,6 & 156 & 12 & 185 & 14,2 & 5189 \\
\hline 2 & 10 & 5 & 38 & 11,9 & 113 & 9,5 & 136 & 11,4 & 170 & 14,3 & 200 & 16,8 & 5190 \\
\hline 2 & 10 & 5 & 44 & 10,3 & 113 & 11 & 136 & 13,2 & 170 & 16,5 & 200 & 19,4 & 5191 \\
\hline 2 & 10 & 5 & 51 & 8,9 & 114 & 12,8 & 136 & 15,3 & 170 & 19,1 & 208 & 23,4 & 5192 \\
\hline 2 & 10 & 5 & 64 & 7,5 & 120 & 16 & 144 & 19,2 & 180 & 24 & 212 & 28,2 & 5193 \\
\hline 2 & 10 & 5 & 76 & 5,3 & 101 & 19 & 121 & 22,8 & 151 & 28,5 & 181 & 34,2 & 5194 \\
\hline 2 & 10 & 5 & 305 & 1,6 & 122 & 76,3 & 146 & 91,5 & 183 & 114,4 & 214 & 133,8 & 5195 \\
\hline 3 & 10 & 5 & 25 & 22,1 & 111 & 5,0 & 139 & 6,3 & 166 & 7,5 & 203 & 9,2 & 5285 \\
\hline 3 & 10 & 5 & 32 & 17,5 & 112 & 6,4 & 140 & 8 & 168 & 9,6 & 212 & 12,1 & 5286 \\
\hline 3 & 10 & 5 & 38 & 17,1 & 130 & 7,6 & 162 & 9,5 & 195 & 11,4 & 226 & 13,2 & 5287 \\
\hline 3 & 10 & 5 & 44 & 15 & 132 & 8,8 & 165 & 11 & 198 & 13,2 & 227 & 15,1 & 5288 \\
\hline 3 & 10 & 5 & 51 & 12,8 & 131 & 10,2 & 164 & 12,8 & 196 & 15,3 & 250 & 19,5 & 5289 \\
\hline 3 & 10 & 5 & 64 & 10,7 & 137 & 12,8 & 171 & 16 & 205 & 19,2 & 233 & 21,8 & 5290 \\
\hline 3 & 10 & 5 & 76 & 7,5 & 114 & 15,2 & 143 & 19 & 171 & 22,8 & 209 & 27,9 & 5291 \\
\hline 3 & 10 & 5 & 305 & 2,1 & 128 & 61 & 160 & 76,3 & 192 & 91,5 & 267 & 127,2 & 5292 \\
\hline 4 & 10 & 5 & 25 & 36,8 & 158 & 4,3 & 184 & 5 & 232 & 6,3 & 283 & 7,7 & 5381 \\
\hline 4 & 10 & 5 & 32 & 27,9 & 151 & 5,4 & 179 & 6,4 & 223 & 8 & 296 & 10,6 & 5382 \\
\hline 4 & 10 & 5 & 38 & 23,7 & 154 & 6,5 & 180 & 7,6 & 225 & 9,5 & 299 & 12,6 & 5383 \\
\hline 4 & 10 & 5 & 44 & 19,2 & 144 & 7,5 & 169 & 8,8 & 211 & 11 & 265 & 13,8 & 5384 \\
\hline 4 & 10 & 5 & 51 & 16,5 & 144 & 8,7 & 168 & 10,2 & 211 & 12,8 & 267 & 16,2 & 5385 \\
\hline 4 & 10 & 5 & 64 & 13,2 & 144 & 10,9 & 169 & 12,8 & 211 & 16 & 269 & 20,4 & 5386 \\
\hline 4 & 10 & 5 & 76 & 10,9 & 141 & 12,9 & 166 & 15,2 & 207 & 19 & 275 & 25,2 & 5387 \\
\hline 4 & 10 & 5 & 305 & 2,6 & 135 & 51,9 & 159 & 61 & 198 & 76,3 & 288 & 110,8 & 5388 \\
\hline
\end{tabular}




\section{DIE SPRINGS}

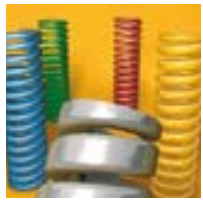

\begin{tabular}{|c|c|c|c|c|c|c|c|c|c|c|c|c|c|}
\hline $\begin{array}{l}\text { Force } \\
\text { class }\end{array}$ & A & B & $L_{0}$ & C & $\mathbf{F}_{\mathbf{1}}$ & $\mathbf{s}_{1}$ & $\mathbf{F}_{\mathbf{2}}$ & $s_{2}$ & $\mathbf{F}_{\mathbf{3}}$ & $s_{3}$ & $\mathbf{F}_{\text {st }}$ & $\mathrm{s}_{\text {st }}$ & Cat. no \\
\hline 1 & 12,5 & 6,3 & 25 & 17,9 & 113 & 6,3 & 134 & 7,5 & 179 & 10 & 236 & 13,2 & 5100 \\
\hline 1 & 12,5 & 6,3 & 32 & 16,4 & 131 & 8,0 & 157 & 9,6 & 210 & 12,8 & 295 & 18 & 5101 \\
\hline 1 & 12,5 & 6,3 & 38 & 13,6 & 129 & 9,5 & 155 & 11,4 & 207 & 15,2 & 286 & 21 & 5102 \\
\hline 1 & 12,5 & 6,3 & 44 & 12,1 & 133 & 11 & 160 & 13,2 & 213 & 17,6 & 290 & 24 & 5103 \\
\hline 1 & 12,5 & 6,3 & 51 & 11,4 & 146 & 12,8 & 174 & 15,3 & 233 & 20,4 & 327 & 28,7 & 5104 \\
\hline 1 & 12,5 & 6,3 & 64 & 9,3 & 149 & 16 & 179 & 19,2 & 238 & 25,6 & 333 & 35,8 & 5105 \\
\hline 1 & 12,5 & 6,3 & 76 & 7,1 & 135 & 19 & 162 & 22,8 & 216 & 30,4 & 303 & 42,7 & 5106 \\
\hline 1 & 12,5 & 6,3 & 89 & 5,4 & 120 & 22,3 & 144 & 26,7 & 192 & 35,6 & 272 & 50,4 & 5107 \\
\hline 1 & 12,5 & 6,3 & 305 & 1,4 & 107 & 76,3 & 128 & 91,5 & 171 & 122,0 & 241 & 172 & 5108 \\
\hline 2 & 12,5 & 6,3 & 25 & 30 & 189 & 6,3 & 225 & 7,5 & 282 & 9,4 & 357 & 11,9 & 5196 \\
\hline 2 & 12,5 & 6,3 & 32 & 24,8 & 198 & 8 & 238 & 9,6 & 298 & 12 & 402 & 16,2 & 5197 \\
\hline 2 & 12,5 & 6,3 & 38 & 21,4 & 203 & 9,5 & 244 & 11,4 & 306 & 14,3 & 400 & 18,7 & 5198 \\
\hline 2 & 12,5 & 6,3 & 44 & 18,5 & 204 & 11 & 244 & 13,2 & 305 & 16,5 & 394 & 21,3 & 5199 \\
\hline 2 & 12,5 & 6,3 & 51 & 15,5 & 198 & 12,8 & 237 & 15,3 & 296 & 19,1 & 397 & 25,6 & 5200 \\
\hline 2 & 12,5 & 6,3 & 64 & 12,1 & 194 & 16 & 232 & 19,2 & 290 & 24 & 392 & 32,4 & 5201 \\
\hline 2 & 12,5 & 6,3 & 76 & 10,2 & 194 & 19 & 233 & 22,8 & 291 & 28,5 & 398 & 39 & 5202 \\
\hline 2 & 12,5 & 6,3 & 89 & 8,4 & 187 & 22,3 & 224 & 26,7 & 281 & 33,4 & 386 & 45,9 & 5203 \\
\hline 2 & 12,5 & 6,3 & 305 & 2,1 & 160 & 76,3 & 192 & 91,5 & 240 & 114,4 & 320 & 152,5 & 5204 \\
\hline 3 & 12,5 & 6,3 & 25 & 42,1 & 211 & 5,0 & 265 & 6,3 & 316 & 7,5 & 413 & 9,8 & 5293 \\
\hline 3 & 12,5 & 6,3 & 32 & 33,2 & 212 & 6,4 & 266 & 8 & 319 & 9,6 & 452 & 13,6 & 5294 \\
\hline 3 & 12,5 & 6,3 & 38 & 29,3 & 223 & 7,6 & 278 & 9,5 & 334 & 11,4 & 428 & 14,6 & 5295 \\
\hline 3 & 12,5 & 6,3 & 44 & 24,6 & 216 & 8,8 & 271 & 11 & 325 & 13,2 & 445 & 18,1 & 5296 \\
\hline 3 & 12,5 & 6,3 & 51 & 19,6 & 200 & 10,2 & 251 & 12,8 & 300 & 15,3 & 437 & 22,3 & 5297 \\
\hline 3 & 12,5 & 6,3 & 64 & 15,0 & 192 & 12,8 & 240 & 16 & 288 & 19,2 & 410 & 27,3 & 5298 \\
\hline 3 & 12,5 & 6,3 & 76 & 13,2 & 201 & 15,2 & 251 & 19 & 301 & 22,8 & 437 & 33,1 & 5299 \\
\hline 3 & 12,5 & 6,3 & 89 & 11,4 & 203 & 17,8 & 254 & 22,3 & 304 & 26,7 & 443 & 38,9 & 5300 \\
\hline 3 & 12,5 & 6,3 & 305 & 2,8 & 171 & 61 & 214 & 76,3 & 256 & 91,5 & 391 & 139,7 & 5301 \\
\hline 4 & 12,5 & 6,3 & 25 & 58,5 & 252 & 4,3 & 293 & 5 & 369 & 6,3 & 474 & 8,1 & 5389 \\
\hline 4 & 12,5 & 6,3 & 32 & 43,9 & 237 & 5,4 & 281 & 6,4 & 351 & 8 & 435 & 9,9 & 5390 \\
\hline 4 & 12,5 & 6,3 & 38 & 36 & 234 & 6,5 & 274 & 7,6 & 342 & 9,5 & 464 & 12,9 & 5391 \\
\hline 4 & 12,5 & 6,3 & 44 & 30,3 & 227 & 7,5 & 267 & 8,8 & 333 & 11 & 427 & 14,1 & 5392 \\
\hline 4 & 12,5 & 6,3 & 51 & 26,2 & 228 & 8,7 & 267 & 10,2 & 335 & 12,8 & 456 & 17,4 & 5393 \\
\hline 4 & 12,5 & 6,3 & 64 & 21,2 & 231 & 10,9 & 271 & 12,8 & 339 & 16 & 445 & 21 & 5394 \\
\hline 4 & 12,5 & 6,3 & 76 & 17,1 & 221 & 12,9 & 260 & 15,2 & 325 & 19 & 451 & 26,4 & 5395 \\
\hline 4 & 12,5 & 6,3 & 89 & 14,5 & 219 & 15,1 & 258 & 17,8 & 323 & 22,3 & 457 & 31,5 & 5396 \\
\hline 4 & 12,5 & 6,3 & 305 & 4,3 & 223 & 51,9 & 262 & 61 & 328 & 76,3 & 479 & 111,3 & 5397 \\
\hline
\end{tabular}




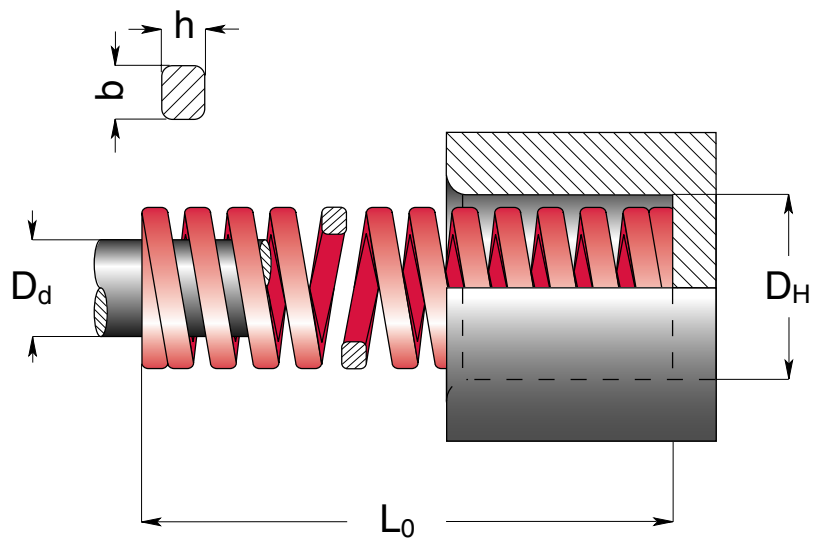

Note: $1 \mathrm{~N}=0,102 \mathrm{Kg}$ (force)

\begin{tabular}{|c|c|c|c|c|c|c|c|c|c|c|c|}
\hline $\mathrm{D}_{\mathrm{H}}$ & $\mathrm{D}_{\mathrm{d}}$ & $L_{0}$ & \multirow[b]{2}{*}{$\mathrm{N} / \mathrm{mm}$} & \multicolumn{2}{|c|}{$\begin{array}{c}20 \% \\
3.000 .000\end{array}$} & \multicolumn{2}{|c|}{$\begin{array}{c}25 \% \\
1.500 .000\end{array}$} & \multicolumn{2}{|c|}{$\begin{array}{c}30 \% \\
\text { Max. Defl. }\end{array}$} & \multicolumn{2}{|c|}{$\begin{array}{c}\text { D } \\
\text { Approx. }\end{array}$} \\
\hline $\mathrm{mm}$ & $\mathrm{mm}$ & $\mathrm{mm}$ & & $\mathrm{mm}$ & $\mathrm{N}$ & $\mathrm{mm}$ & $\mathrm{N}$ & $\mathrm{mm}$ & $\mathrm{N}$ & $\mathrm{mm}$ & $\mathrm{N}$ \\
\hline \multirow{7}{*}{10} & \multirow{7}{*}{5} & 25 & 22.1 & 5.0 & 111 & 6.3 & 139 & 7.5 & 168 & 9.2 & 203 \\
\hline & & 32 & 17.5 & 6.4 & 112 & 8.0 & 140 & 9.6 & 168 & 12.1 & 212 \\
\hline & & 39 & 17.1 & 7.6 & 130 & 9.5 & 162 & 11.4 & 195 & 13.2 & 226 \\
\hline & & 44 & 15.0 & 8.8 & 132 & 11.0 & 165 & 13.2 & 198 & 15.1 & 227 \\
\hline & & 51 & 12.8 & 10.2 & 131 & 12.8 & 164 & 15.3 & 198 & 19.5 & 250 \\
\hline & & 64 & 10.7 & 12.8 & 137 & 16.0 & 171 & 19.2 & 205 & 21.8 & 233 \\
\hline & & 76 & 7.5 & 15.2 & 114 & 19.0 & 143 & 22.8 & 171 & 27.9 & 200 \\
\hline \multicolumn{2}{|c|}{$1.9 \times 1.5$} & 305 & 2.1 & 61.0 & 128 & 76.3 & 160 & 91.5 & 192 & 127.2 & 267 \\
\hline \multirow{9}{*}{12.5} & \multirow{9}{*}{6.3} & 25 & 42.1 & 5.0 & 211 & 6.3 & 265 & 7.5 & 316 & 9.8 & 413 \\
\hline & & 32 & 33.2 & 6.4 & 212 & 8.0 & 266 & 9.6 & 319 & 13.6 & 452 \\
\hline & & 39 & 29.3 & 7.6 & 223 & 9.5 & 278 & 11.4 & 334 & 14.6 & 428 \\
\hline & & 44 & 24.6 & 8.8 & 216 & 11.0 & 271 & 13.2 & 325 & 18.1 & 445 \\
\hline & & 51 & 19.6 & 10.2 & 200 & 12.8 & 251 & 15.3 & 300 & 22.3 & 437 \\
\hline & & 64 & 15.0 & 12.8 & 192 & 16.0 & 240 & 19.2 & 289 & 27.3 & 410 \\
\hline & & 76 & 13.2 & 15.2 & 201 & 19.0 & 251 & 22.8 & 301 & 33.1 & 437 \\
\hline & & 89 & 11.4 & 17.8 & 203 & 22.3 & 254 & 26.7 & 304 & 38.9 & 443 \\
\hline & & 102 & 8.4 & 20.4 & 171 & 25.5 & 214 & 30.6 & 257 & 43.8 & 368 \\
\hline \multicolumn{2}{|c|}{$2.4 \times 1.9$} & 305 & 2.8 & 61.0 & 171 & 76.3 & 214 & 91.5 & 256 & 139.7 & 391 \\
\hline \multirow{10}{*}{16} & \multirow{10}{*}{8} & 25 & 75.7 & 5.0 & 379 & 6.3 & 477 & 7.5 & 568 & 8.4 & 636 \\
\hline & & 32 & 52.8 & 6.4 & 338 & 8.0 & 422 & 9.6 & 507 & 10.5 & 554 \\
\hline & & 39 & 48.5 & 7.6 & 369 & 9.5 & 461 & 11.4 & 563 & 13.6 & 660 \\
\hline & & 44 & 42.8 & 8.8 & 377 & 11.0 & 471 & 13.2 & 565 & 15.9 & 681 \\
\hline & & 51 & 37.1 & 10.2 & 378 & 12.8 & 475 & 15.3 & 568 & 18.9 & 701 \\
\hline & & 64 & 30.3 & 12.8 & 389 & 16.0 & 495 & 19.2 & 582 & 24.9 & 754 \\
\hline & & 76 & 25.7 & 15.2 & 391 & 19.0 & 498 & 22.8 & 588 & 29.2 & 750 \\
\hline & & 89 & 21.7 & 17.8 & 398 & 22.3 & 494 & 26.7 & 579 & 34.5 & 749 \\
\hline & & 102 & 19.3 & 20.4 & 394 & 25.5 & 492 & 30.6 & 591 & 39.1 & 755 \\
\hline & & 115 & 15.7 & 23.0 & 361 & 28.8 & 452 & 34.5 & 542 & 44.0 & 691 \\
\hline \multicolumn{2}{|c|}{$3.1 \times 2.5$} & 305 & 7.1 & 61.0 & 433 & 76.3 & 542 & 91.5 & 660 & 103.6 & 736 \\
\hline \multirow{14}{*}{20} & \multirow{14}{*}{10} & 25 & 216 & 5.0 & 1080 & 6.3 & 1361 & 7.5 & 1620 & 8.3 & 1793 \\
\hline & & 32 & $16 \mathrm{~B}$ & 6.4 & 1075 & 8.0 & 1344 & 9.6 & 1613 & 10.9 & 1831 \\
\hline & & 39 & 129 & 7.6 & 980 & 9.5 & 1226 & 11.4 & 1471 & 12.5 & 1613 \\
\hline & & 44 & 112 & 8.8 & 986 & 11.0 & 1232 & 13.2 & 1478 & 15.0 & 1680 \\
\hline & & 51 & 94.0 & 10.2 & 969 & 12.8 & 1203 & 15.3 & 1438 & 17.6 & 1654 \\
\hline & & 64 & 72.1 & 12.8 & 923 & 16.0 & 1154 & 19.2 & 1384 & 22.6 & 1629 \\
\hline & & 76 & 59.7 & 15.2 & 907 & 19.0 & 1134 & 22.8 & 1361 & 27.5 & 1642 \\
\hline & & 89 & 50.5 & 17.8 & 899 & 22.3 & 1126 & 26.7 & 1348 & 31.7 & 1601 \\
\hline & & 102 & 44.2 & 20.4 & 902 & 25.5 & 1127 & 30.6 & 1353 & 37.5 & 1669 \\
\hline & & 115 & 38.4 & 23.0 & 883 & $2 B .8$ & 1106 & 34.5 & 1325 & 42.6 & 1636 \\
\hline & & 127 & 34.1 & 25.4 & 968 & 31.8 & 1084 & 38.1 & 1299 & 45.5 & 1552 \\
\hline & & 139 & 31.0 & 28.0 & 969 & 35.0 & 1085 & 42.0 & 1302 & 50.1 & 1553 \\
\hline & & 152 & 28.2 & 30.4 & 857 & 38.0 & 1072 & 45.6 & 1286 & 55.8 & 1574 \\
\hline & & 305 & 15.0 & 61.0 & 915 & 76.3 & 1145 & 91.5 & 1373 & 114.1 & 1712 \\
\hline
\end{tabular}




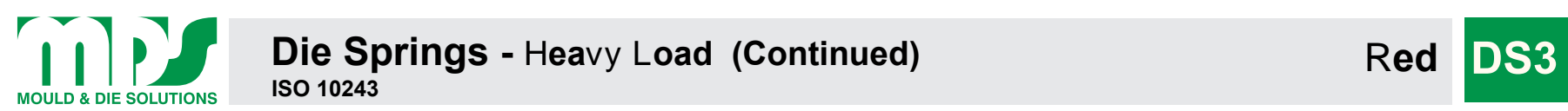

Note: $1 \mathrm{~N}=0,102 \mathrm{Kg}$ (force)

\begin{tabular}{|c|c|c|c|c|c|c|c|c|c|c|c|}
\hline $\mathrm{D}_{\mathrm{H}}$ & $D_{d}$ & $\mathrm{~L}_{0}$ & Rate & \multicolumn{2}{|c|}{$\begin{array}{c}20 \% \\
3.000 .000\end{array}$} & \multicolumn{2}{|c|}{$\begin{array}{c}25 \% \\
1.500 .000\end{array}$} & \multicolumn{2}{|c|}{$\begin{array}{c}30 \% \\
\text { Max. Defl. }\end{array}$} & \multicolumn{2}{|c|}{$\begin{array}{c}\text { A } \\
\text { Approx. }\end{array}$} \\
\hline $\mathrm{mm}$ & $\mathrm{mm}$ & $\mathrm{mm}$ & $\mathrm{N} / \mathrm{mm}$ & $\mathrm{mm}$ & $\mathrm{N}$ & $\mathrm{mm}$ & $\mathrm{N}$ & $\mathrm{mm}$ & $\mathrm{N}$ & $\mathrm{mm}$ & $\mathrm{N}$ \\
\hline \multirow{15}{*}{25} & \multirow{15}{*}{12.5} & 25 & 375 & 5.0 & 1875 & 6.3 & 2363 & 7.5 & 2813 & 8.5 & 3189 \\
\hline & & 32 & 297 & 6.4 & 1901 & 8.0 & 2376 & 9.6 & 2851 & 11.0 & 3267 \\
\hline & & 38 & 219 & 7.6 & 1664 & 9.5 & 2081 & 11.4 & 2497 & 12.6 & 2759 \\
\hline & & 44 & 197 & B. $B$ & 1646 & 11.0 & 2057 & 13.2 & 2468 & 14.8 & 2768 \\
\hline & & 51 & 156 & 10.2 & 1591 & 12.8 & 1997 & 15.3 & 2397 & 17.9 & 2792 \\
\hline & & 64 & 123 & 12.8 & 1574 & 16.0 & 1968 & 19.2 & 2362 & 23.1 & 2841 \\
\hline & & 76 & 99.0 & 15.2 & 1505 & 19.0 & 1881 & 22.8 & 2257 & 26.3 & 2604 \\
\hline & & 89 & 84.0 & 17.8 & 1495 & 22.3 & 1873 & 26.7 & 2243 & 30.5 & 2562 \\
\hline & & 102 & 73.0 & 20.4 & 1489 & 25.5 & 1862 & 30.6 & 2234 & 37.3 & 2723 \\
\hline & & 115 & 65.0 & 23.0 & 1495 & 28.8 & 1872 & 34.5 & 2243 & 41.9 & 2724 \\
\hline & & 127 & 57.7 & 25.4 & 1466 & 31.8 & 1835 & 38.1 & 2198 & 46.2 & 2668 \\
\hline & & 139 & 52.7 & 28.0 & 1476 & 35.0 & 1845 & 42.0 & 2213 & 49.3 & 2598 \\
\hline & & 152 & 47.8 & 30.4 & 1453 & 38.0 & 1816 & 45.6 & 2180 & 55.7 & 2662 \\
\hline & & 178 & 41.0 & 35.6 & 1460 & 44.5 & 1825 & 53.4 & 2189 & 65.1 & 2669 \\
\hline & & 203 & 35.8 & 40.6 & 1453 & 50.8 & 1819 & 60.9 & 2180 & 74.5 & 2667 \\
\hline & 4.2 & 305 & 22.9 & 61.0 & 1397 & 76.3 & 1747 & 91.5 & 2095 & 110.2 & 2524 \\
\hline & & 38 & 398 & 7.6 & 2949 & 9.5 & 3686 & 11.4 & 4423 & 12.5 & 4950 \\
\hline & & 44 & 324 & 8.8 & 2851 & 11.0 & 3564 & 13.2 & 4277 & 14.9 & 4928 \\
\hline & & 51 & 272 & 10.2 & 2774 & 12.8 & 3482 & 15.3 & 4162 & 17.8 & 4942 \\
\hline & & 64 & 212 & 12.8 & 2714 & 16.0 & 3392 & 19.2 & 4070 & 22.4 & 4749 \\
\hline & & 76 & 172 & 15.2 & 2614 & 19.0 & 3269 & 22.8 & 3922 & 26.1 & 4489 \\
\hline & & 89 & 141 & 17.8 & 2510 & 22.3 & 3144 & 26.7 & 3765 & 30.8 & 4343 \\
\hline 32 & 16 & 102 & 122 & 20.4 & 2489 & 25.5 & 3111 & 30.6 & 3733 & 36.8 & 4490 \\
\hline (1) & 10 & 115 & 107 & 23.0 & 2461 & 28.8 & 3082 & 34.5 & 3692 & 41.4 & 4430 \\
\hline & & 127 & 93.0 & 25.4 & 2362 & 31.8 & 2957 & 38.1 & 3543 & 44.4 & 4129 \\
\hline & & 139 & 86.0 & 28.0 & $240 B$ & 35.0 & 3010 & 42.0 & 3612 & 48.5 & 4171 \\
\hline & & 152 & 78.0 & 30.4 & 2371 & 38.0 & 2964 & 45.6 & 3557 & 54.8 & 4274 \\
\hline & & 178 & 67.2 & 35.6 & 2392 & 44.5 & 2990 & 53.4 & 3588 & 63.6 & 4274 \\
\hline & & 203 & 59.1 & 40.6 & 2399 & 50.8 & 3002 & 60.9 & 3599 & 72.5 & 4285 \\
\hline & & 254 & 46.4 & 50.8 & 2357 & 63.5 & 2946 & 76.2 & 3536 & 92.8 & 4308 \\
\hline & 5.4 & 305 & 38.0 & 61.0 & 2318 & 76.3 & 2899 & 91.5 & 3477 & 111. B & 4248 \\
\hline & & 51 & 350 & 10.2 & 3570 & 12.8 & 4480 & 15.3 & 5355 & 17.0 & 5960 \\
\hline & & 64 & 269 & 12.8 & 3443 & 16.0 & 4304 & 19.2 & 5165 & 21.9 & 5991 \\
\hline & & 76 & 219 & 15.2 & 3329 & 19.0 & 4161 & 22.8 & 4993 & 26.7 & 6847 \\
\hline & & 89 & 190 & 17.8 & 3392 & 22.3 & 4237 & 26.7 & 5073 & 31.3 & 5947 \\
\hline & & 102 & 163 & 20.4 & 3325 & 25.5 & 4157 & 30.6 & 4998 & 37.1 & 6047 \\
\hline 40 & 20 & 115 & 142 & 23.0 & 3266 & 28.8 & 4090 & 34.5 & 4899 & 41.0 & 5922 \\
\hline & & 127 & $12 \mathrm{~B}$ & 25.4 & 3251 & 31.8 & 4070 & 38.1 & 4877 & 46.5 & 5962 \\
\hline & & 139 & 115 & 28.0 & 3220 & 35.0 & 4025 & 42.0 & 4830 & 53.1 & 6107 \\
\hline & & 152 & 105 & 30.4 & 3192 & 38.0 & 3990 & 45.6 & 4798 & 56.1 & 6991 \\
\hline & & 178 & 89 & 35.6 & 3168 & 44.5 & 3961 & 53.4 & 4753 & 67.4 & 6999 \\
\hline & & 203 & 77 & 40.6 & 3126 & 50.8 & 3912 & 60.9 & 4689 & 76.2 & 5967 \\
\hline & & 254 & 61 & 50.8 & 3099 & 63.5 & 3974 & 76.2 & 4648 & 96.2 & 5968 \\
\hline & 6.2 & 305 & 51 & 61.0 & 3111 & 76.3 & 3891 & 91.5 & 4687 & 114.8 & 5955 \\
\hline & & 64 & 413 & 12.8 & 5296 & 16.0 & 6609 & 19.2 & 7930 & 22.4 & 9251 \\
\hline & & 76 & 339 & 15.2 & 5153 & 19.0 & 6441 & 22.8 & 7729 & 26.5 & 8984 \\
\hline & & 89 & 288 & 17.8 & 5126 & 22.3 & 6422 & 26.7 & 7690 & 31.5 & 9072 \\
\hline & & 102 & 245 & 20.4 & 4998 & 25.5 & 6248 & 30.6 & 7497 & 37.6 & 9212 \\
\hline & & 115 & 215 & 23.0 & 4945 & 28.8 & 6192 & 34.5 & 7418 & 42.7 & 9181 \\
\hline 50 & 25 & 127 & 192 & 25.4 & 4877 & 31.8 & 6106 & 39.1 & 7315 & 47.5 & 9120 \\
\hline & & 139 & 168 & 28.0 & 4704 & 35.0 & 5980 & 42.0 & 7056 & 51.8 & 8702 \\
\hline & & 152 & 154 & 30.4 & 4692 & 38.0 & 5952 & 45.6 & 7022 & 57.8 & 8901 \\
\hline & & 178 & 134 & 35.6 & 4770 & 44.5 & 5963 & 53.4 & 7156 & 68.5 & 9179 \\
\hline & & 203 & 117 & 40.6 & 4750 & 50.8 & 5944 & 60.9 & 7125 & 77.6 & 9079 \\
\hline & & 254 & 89 & 50.8 & 4521 & 63.5 & 5652 & 76.2 & 6792 & 97.9 & 8713 \\
\hline 11. & 7.6 & 305 & 73 & 61.0 & 4453 & 76.3 & 5570 & 91.5 & 6690 & 120.7 & 8911 \\
\hline & & 76 & $61 \mathrm{~B}$ & 15.2 & 9394 & 19.0 & 11742 & 22.8 & 14090 & 24.7 & 15265 \\
\hline & & 89 & 515 & 17.8 & 9167 & 22.3 & 11485 & 26.7 & 13751 & 30.0 & 15450 \\
\hline & & 102 & 438 & 20.4 & 8935 & 25.5 & 11169 & 30.6 & 13403 & 35.1 & 15374 \\
\hline & & 115 & 370 & 23.0 & 8510 & 28.8 & 10666 & 34.5 & 12765 & 37.5 & 13875 \\
\hline 63 & 38 & 127 & 333 & 25.4 & 8458 & 31.8 & 10589 & 38.1 & 12687 & 45.9 & 15285 \\
\hline & & 152 & 269 & 30.4 & 8178 & 38.0 & 10222 & 45.6 & 12266 & 56.5 & 15199 \\
\hline & & 178 & 226 & 35.6 & 9046 & 44.5 & 10067 & 53.4 & 12068 & 66.8 & 15097 \\
\hline & & 203 & 198 & 40.6 & 8039 & 50.8 & $1006 \mathrm{~B}$ & 60.9 & 12058 & 78.8 & 15602 \\
\hline & & 254 & 155 & 50.8 & 7874 & 63.5 & 9843 & 76.2 & 11811 & 101.7 & 15763 \\
\hline & & 305 & $12 \mathrm{~B}$ & 61.0 & 7808 & 76.3 & 9766 & 91.5 & 11712 & 122.4 & 15667 \\
\hline & & & & & & & & & & & \\
\hline
\end{tabular}


As per the equations displayed in Appendix A, the theoretical maximum spring deflections are:

$>10.43 \mathrm{~mm}$ at maximum dorsiflexion of $13^{\circ}$ (during CDF).

$>2.33 \mathrm{~mm}$ at maximum plantarflexion of $6^{\circ}$ (during CPF).

Thus each spring in each set will undergo the following maximum expected deflection as a percentage of free length:

> Pseudo-plantarflexor: $10.43 \mathrm{~mm}$ deflection $/ 38 \mathrm{~mm}$ free length $=27.4 \%$ deflection.

$>$ Pseudo-dorsiflexor: $2.33 \mathrm{~mm}$ deflection $/ 25 \mathrm{~mm}$ free length $=9.3 \%$ deflection.

According to the catalogue on the previous page, the expected spring lifetime based on maximum expected deflection per cycle is thus:

$>$ Pseudo-plantarflexor $(4.0 \mathrm{~mm} \times 3.3 \mathrm{~mm})<1.5$ million cycle.

$>$ Pseudo-dorsiflexor $(2.4 \mathrm{~mm} \times 1.9 \mathrm{~mm})>3$ million cycles.

At an estimated average 5000 steps per day ( 2500 per foot per day), the expected spring lifetime is therefore:

$>$ Pseudo-plantarflexors: 1500000 cycles $/ 2500$ steps $=600$ days $=1.7$ years.

$>$ Pseudo-dorsiflexors: 3000000 cycles $/ 2500$ steps $=1200$ days $=3.3$ years.

The pseudo-plantarflexors are therefore the limiting factor, and will need to be replaced every one and a half years. 
Appendix D

AJP Drawings 


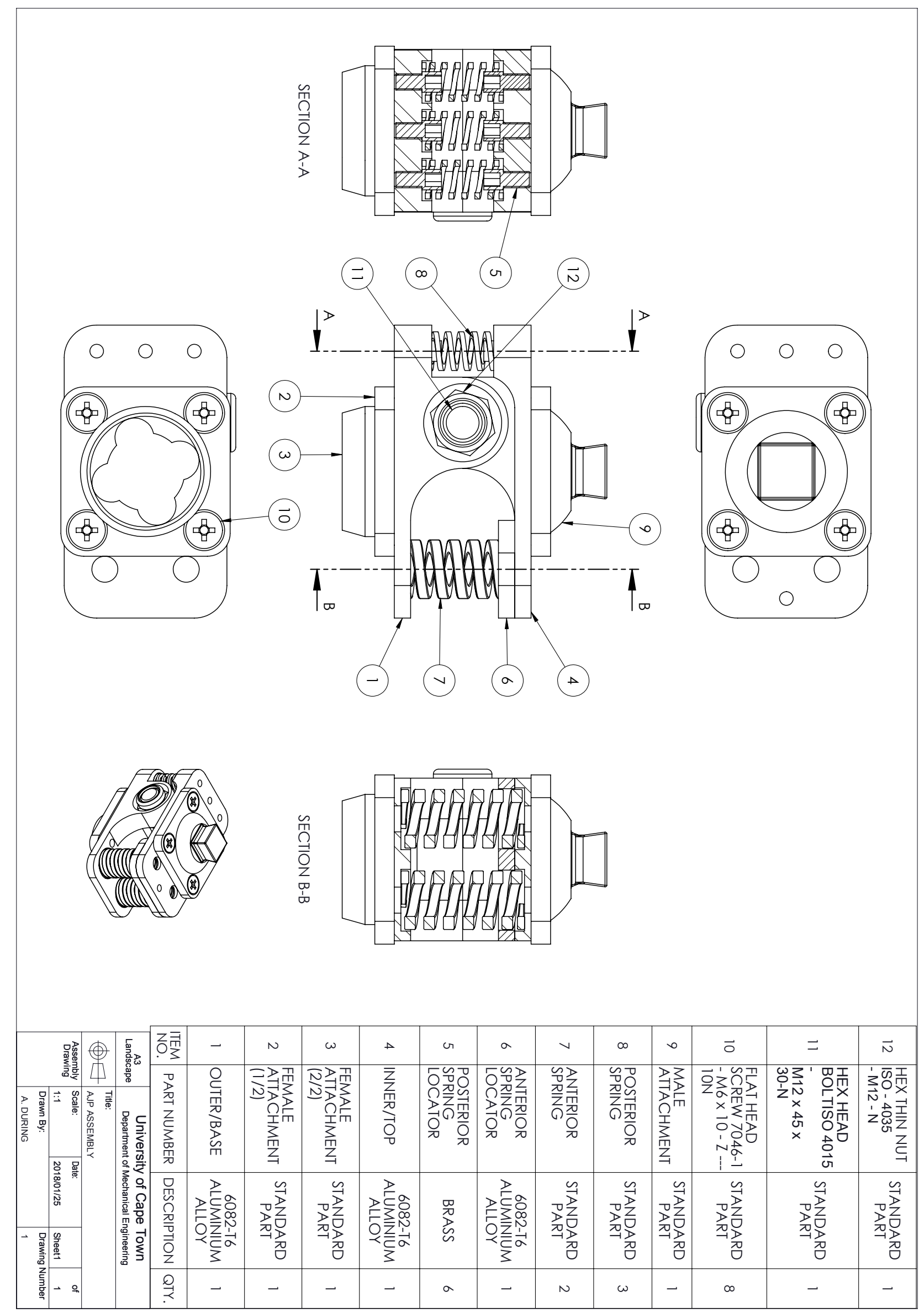




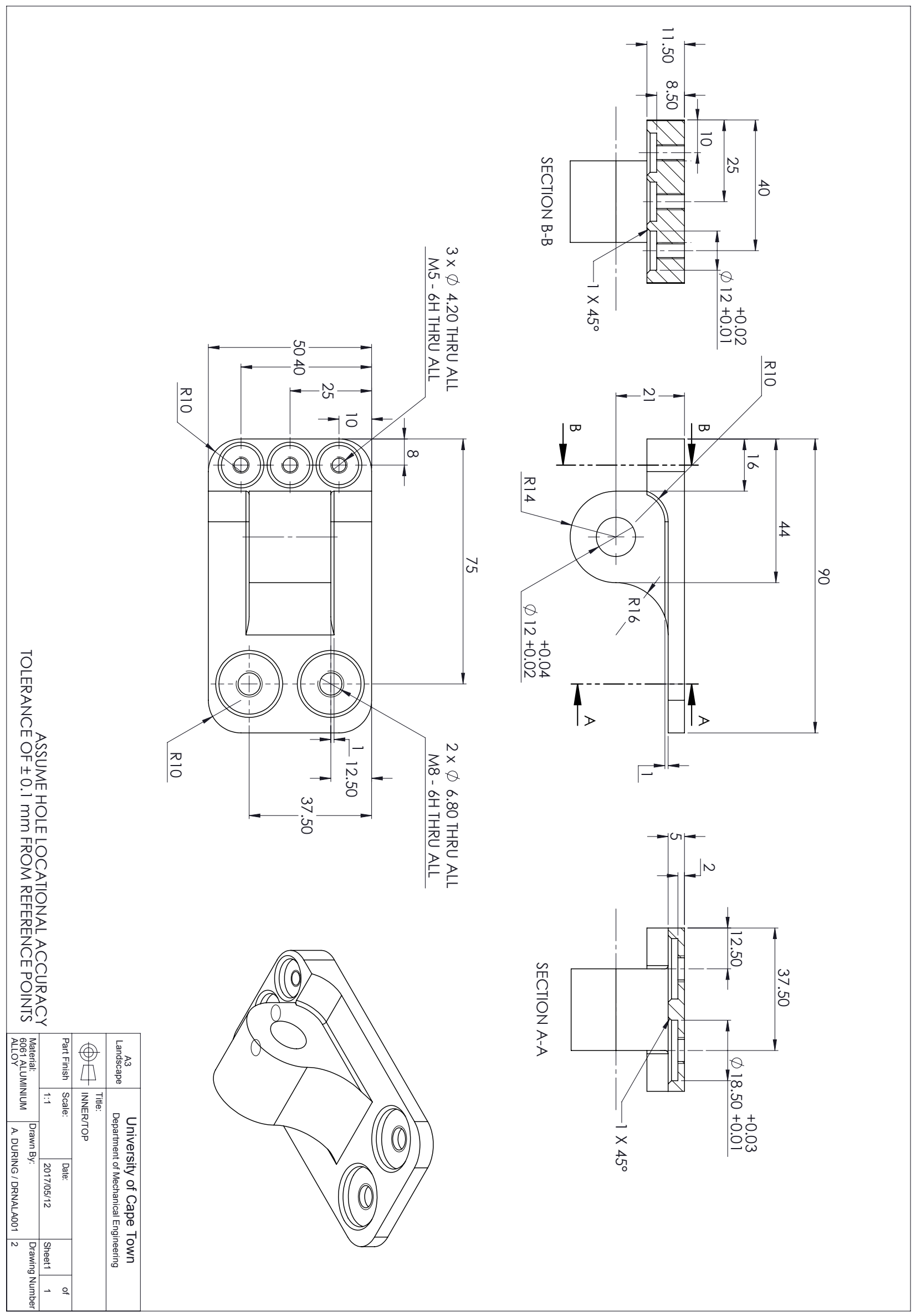




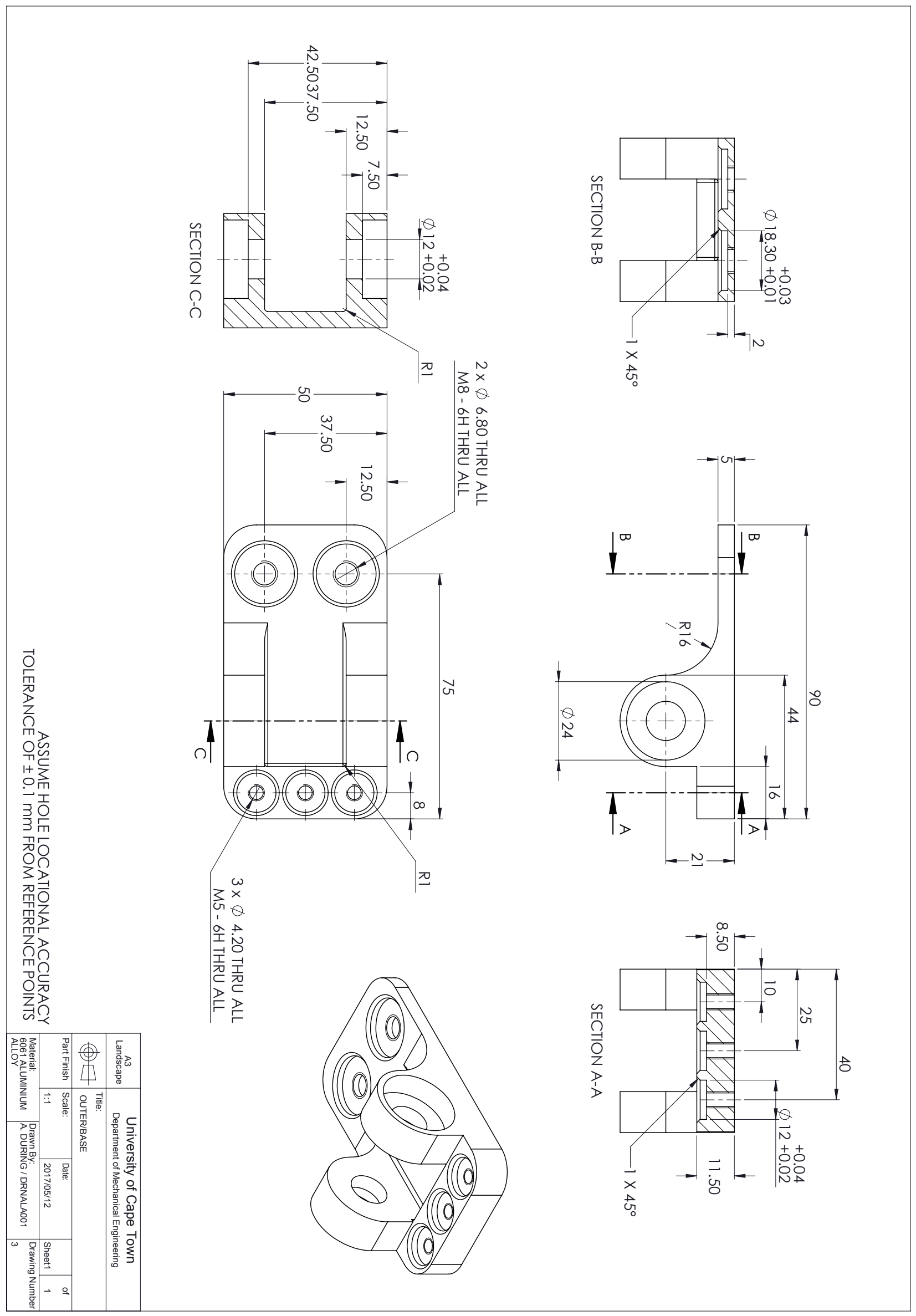




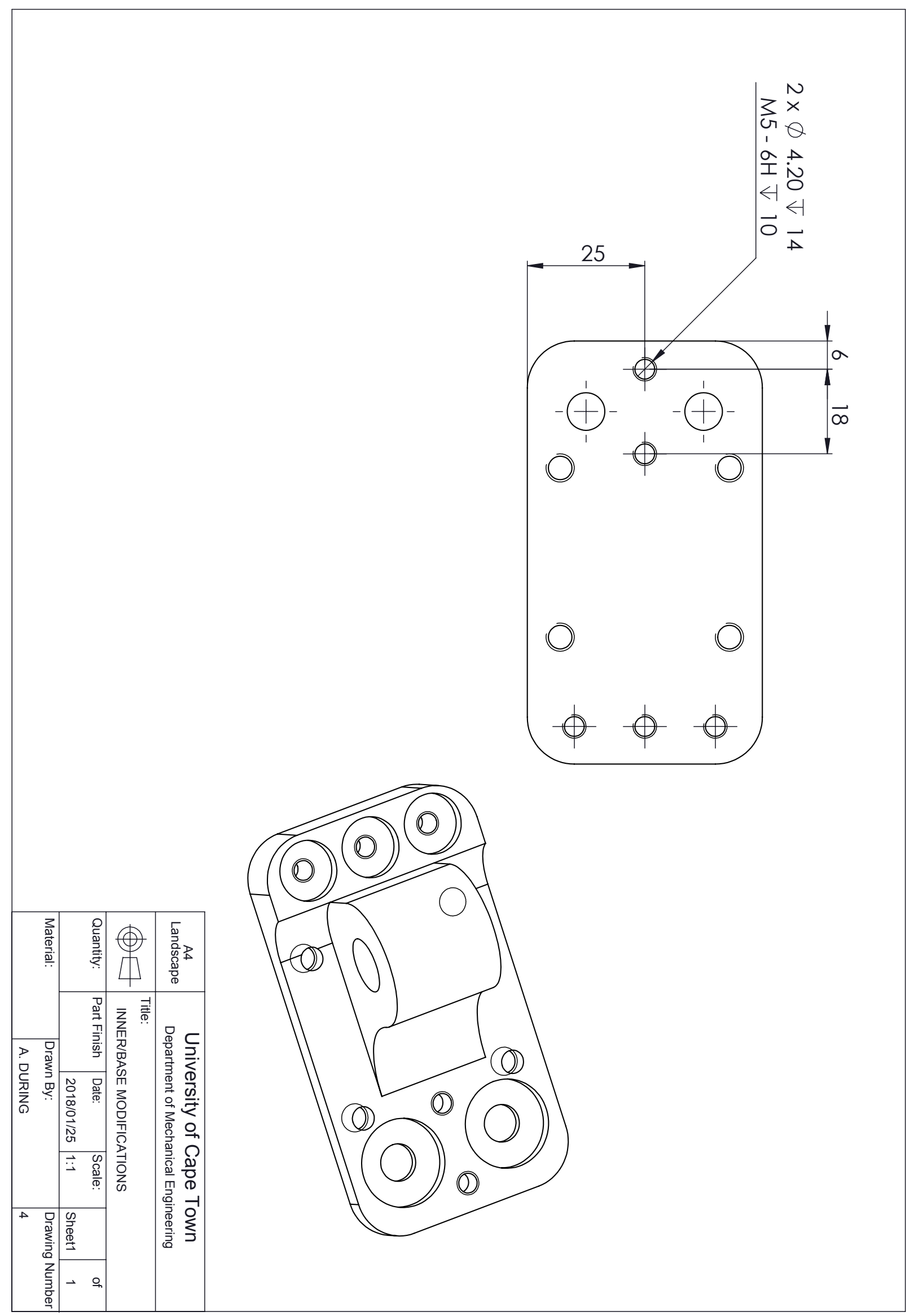




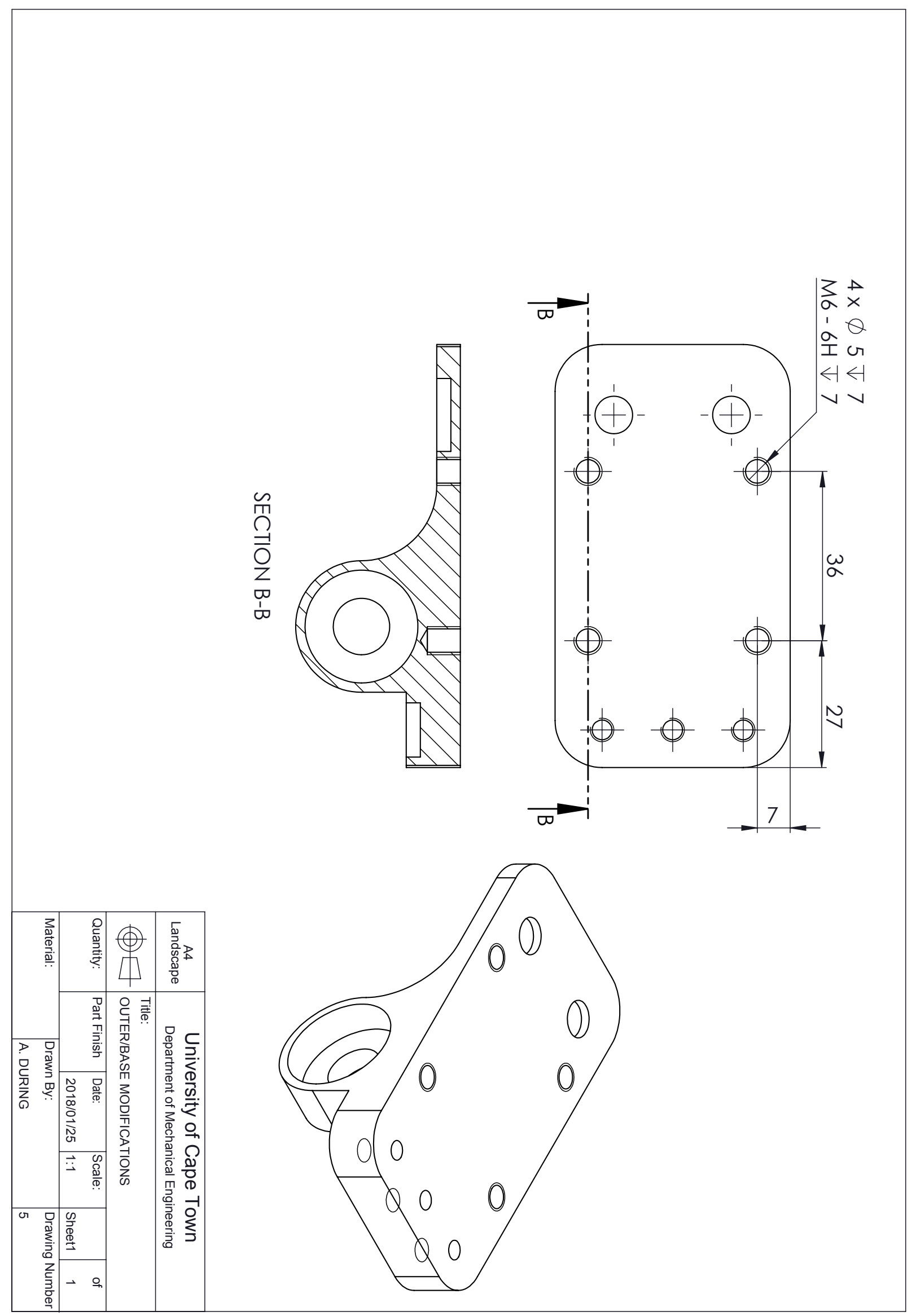




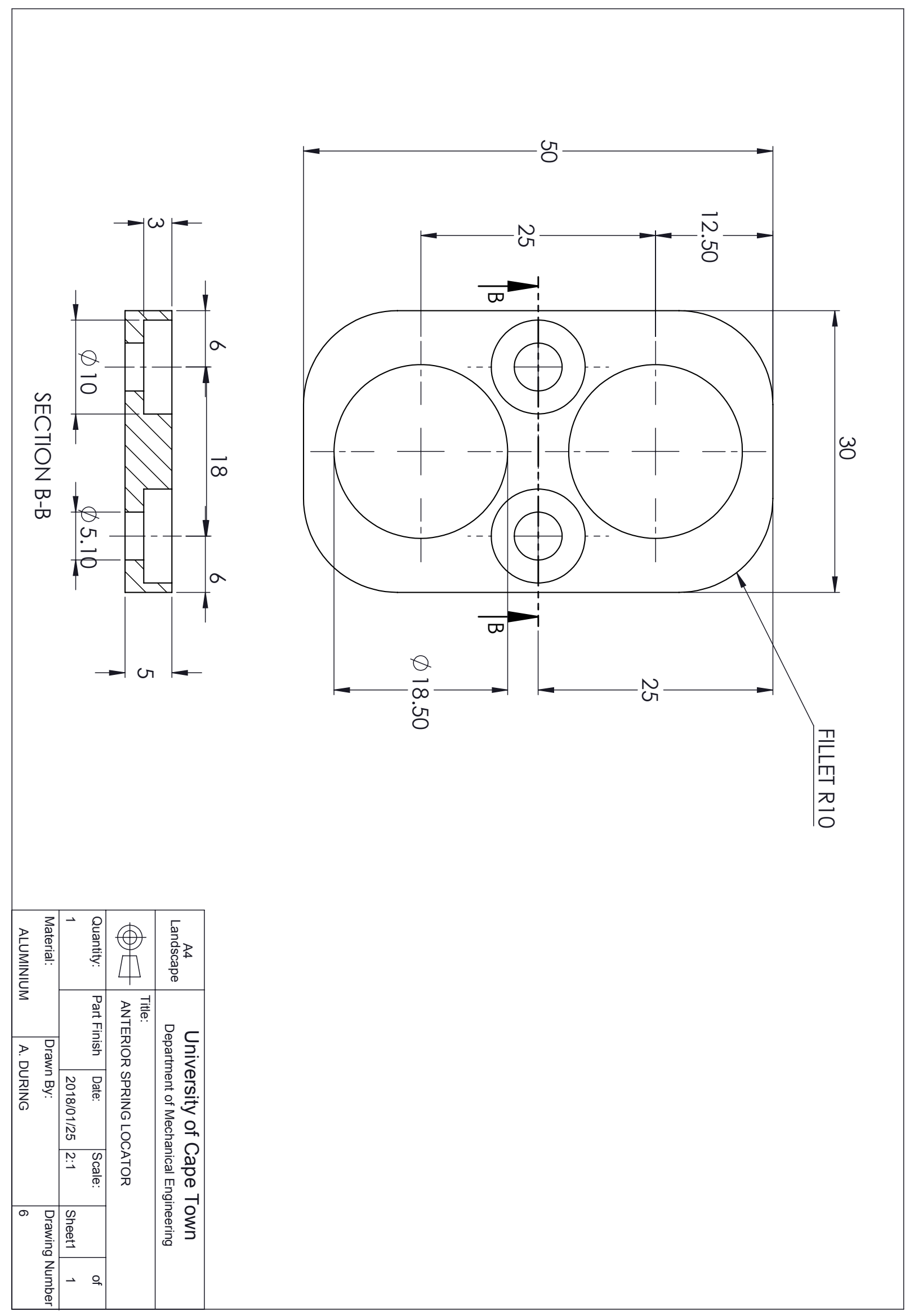




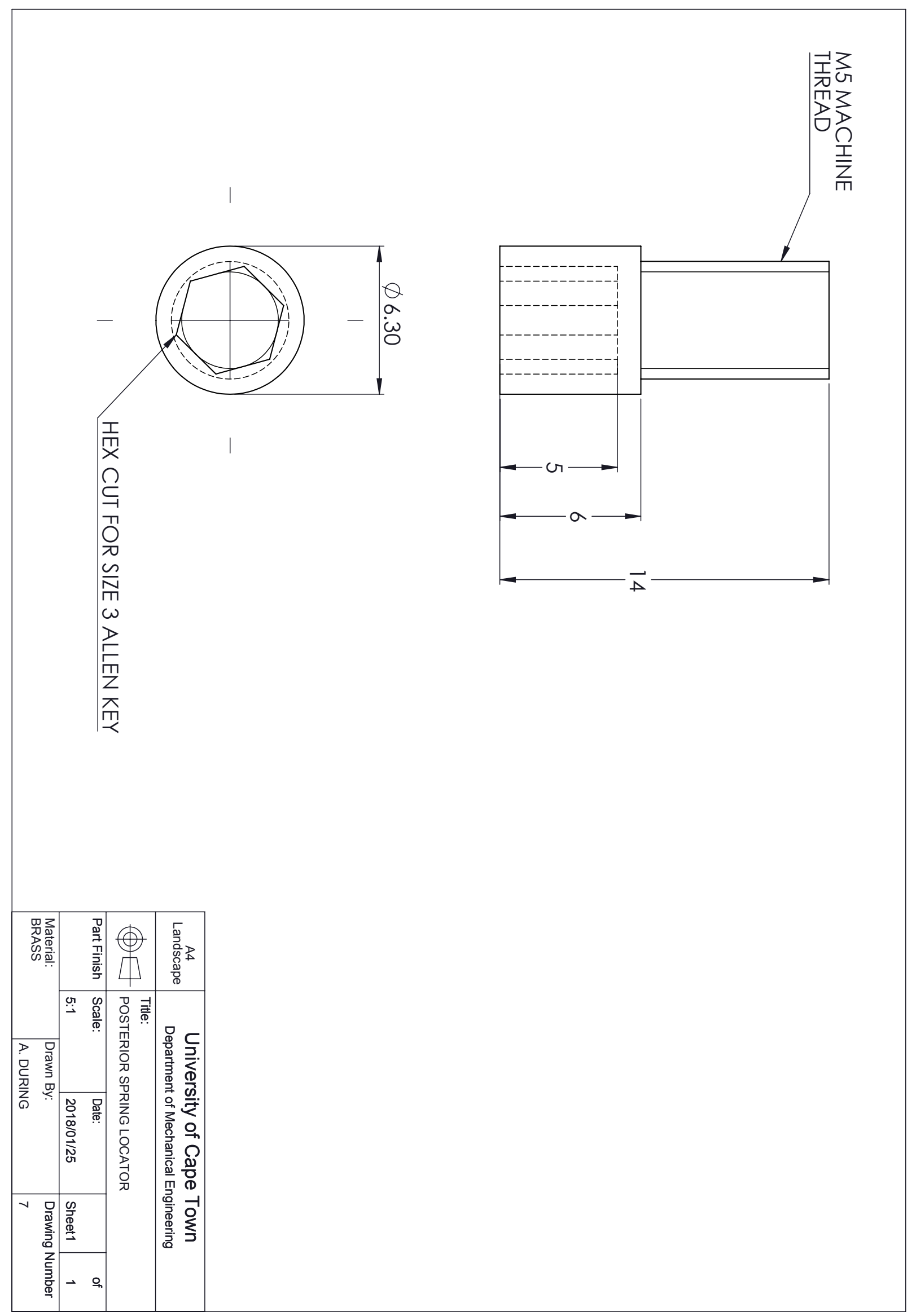




\section{Appendix E}

DPP Drawings

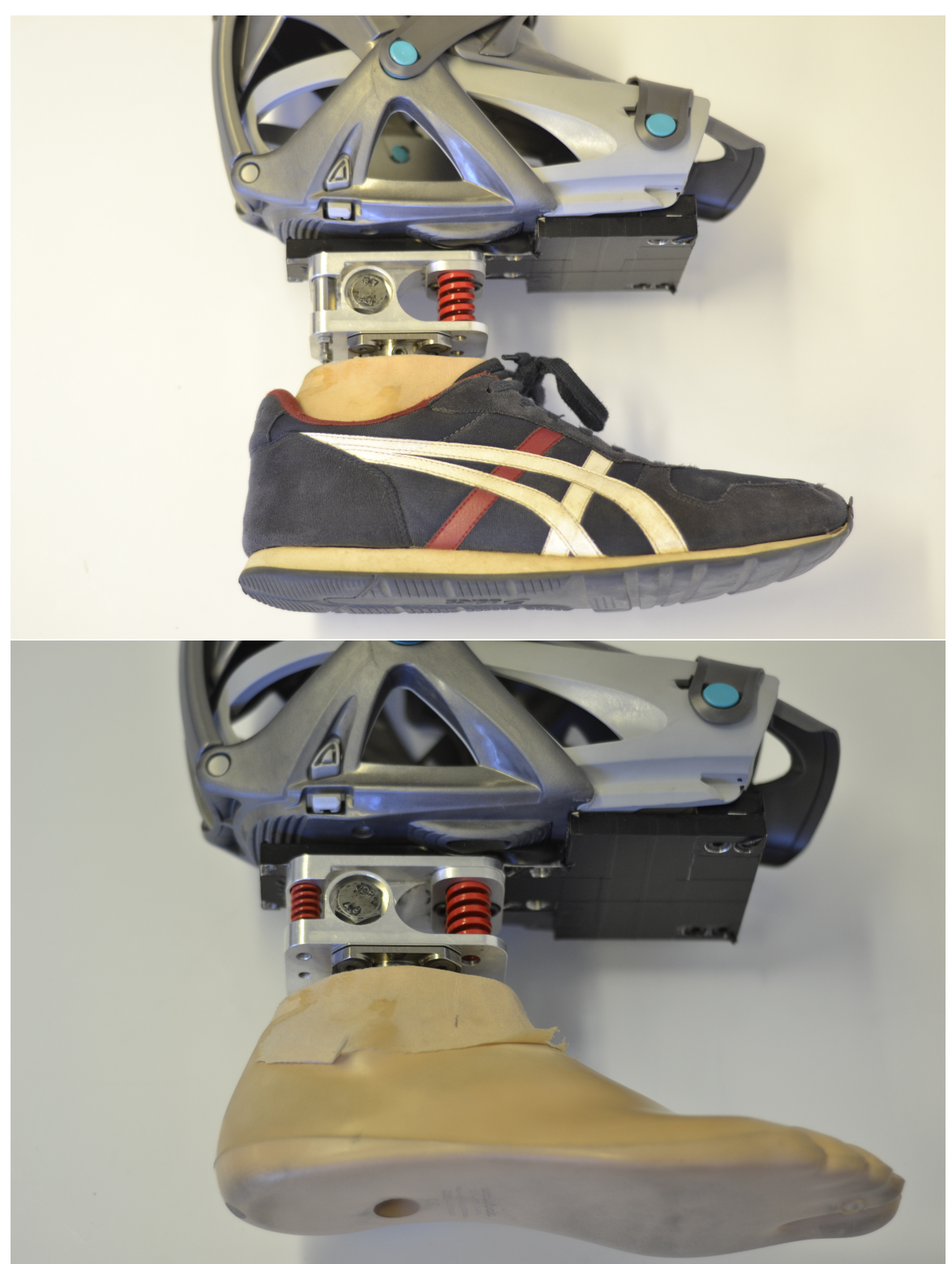




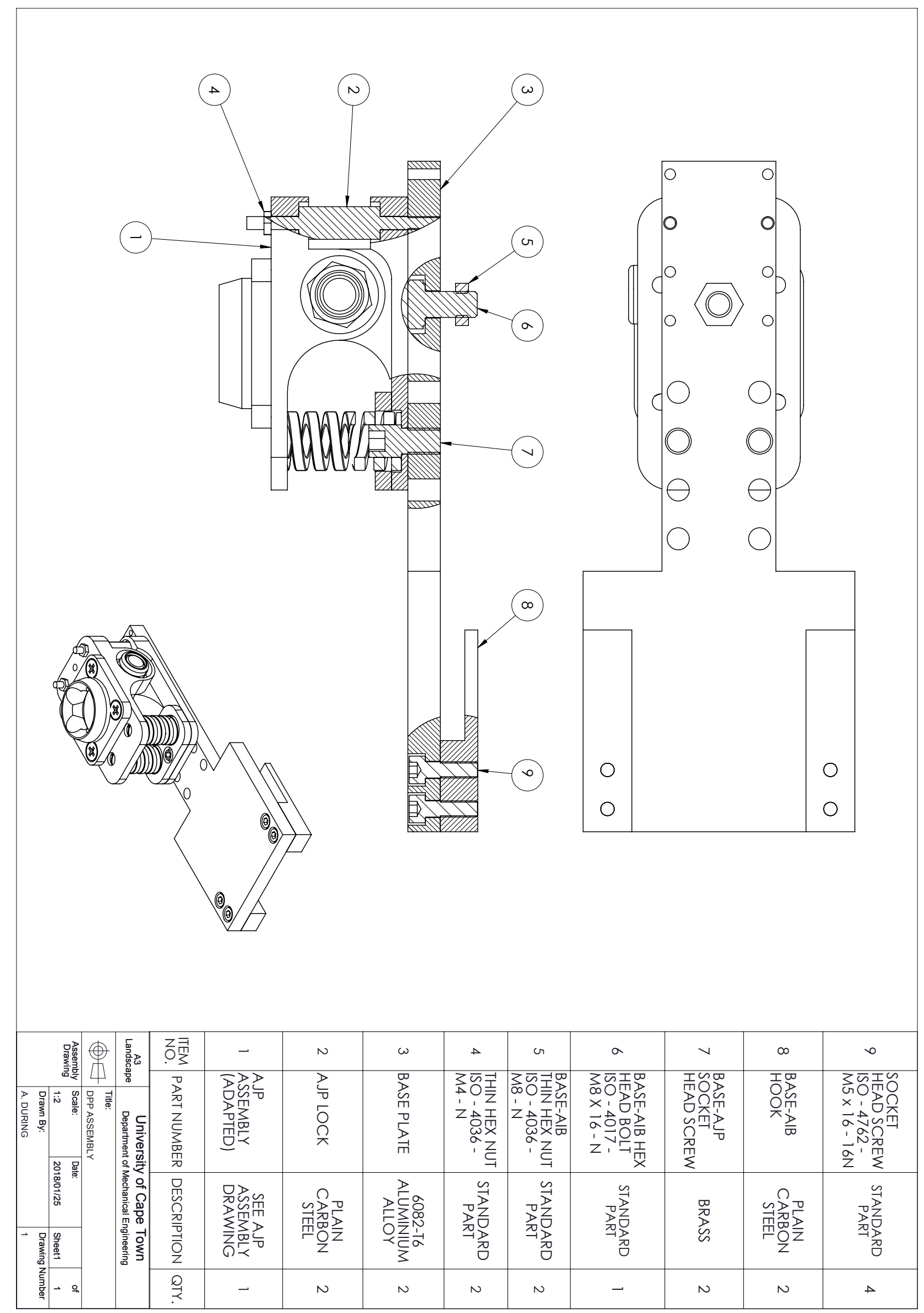




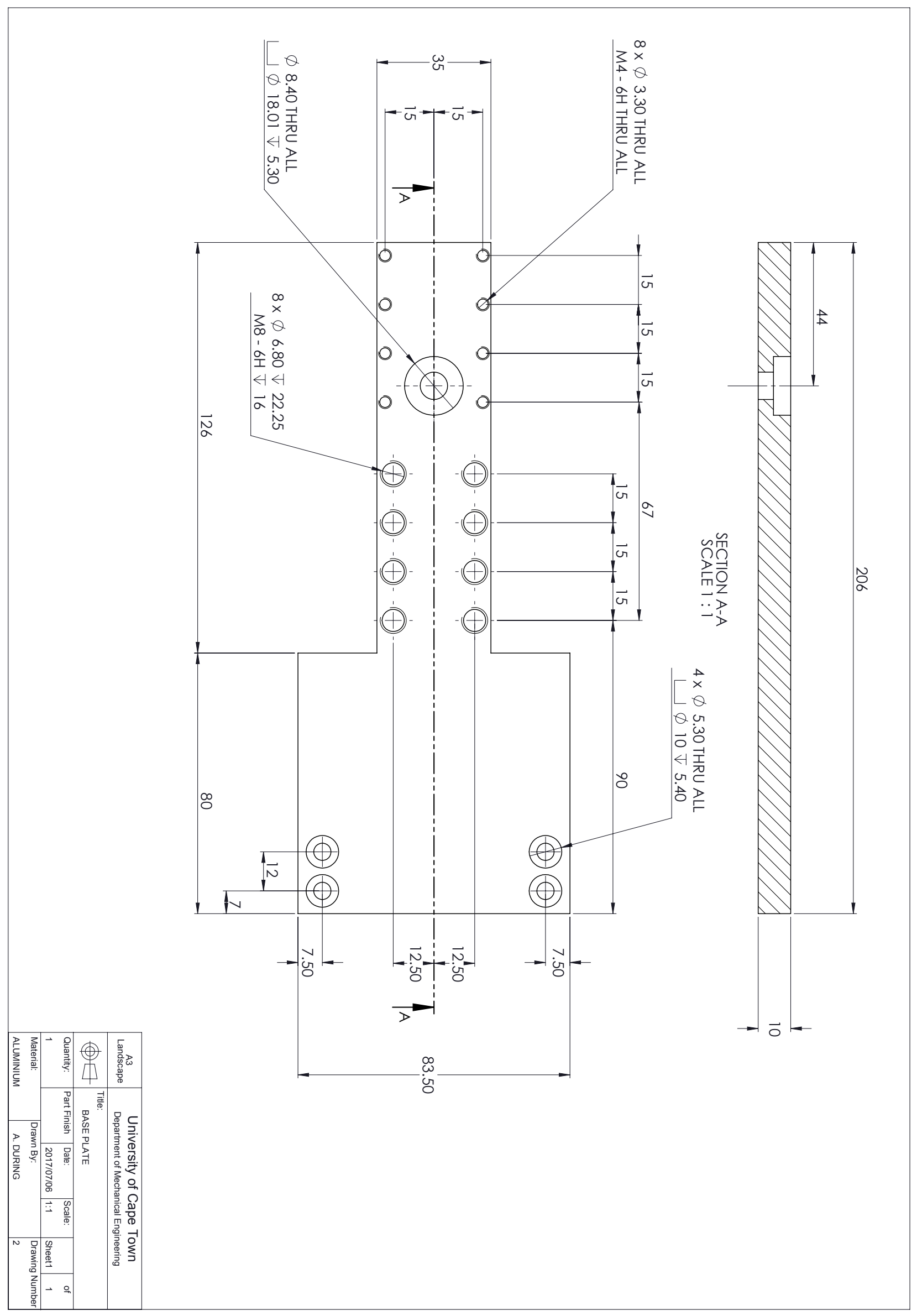




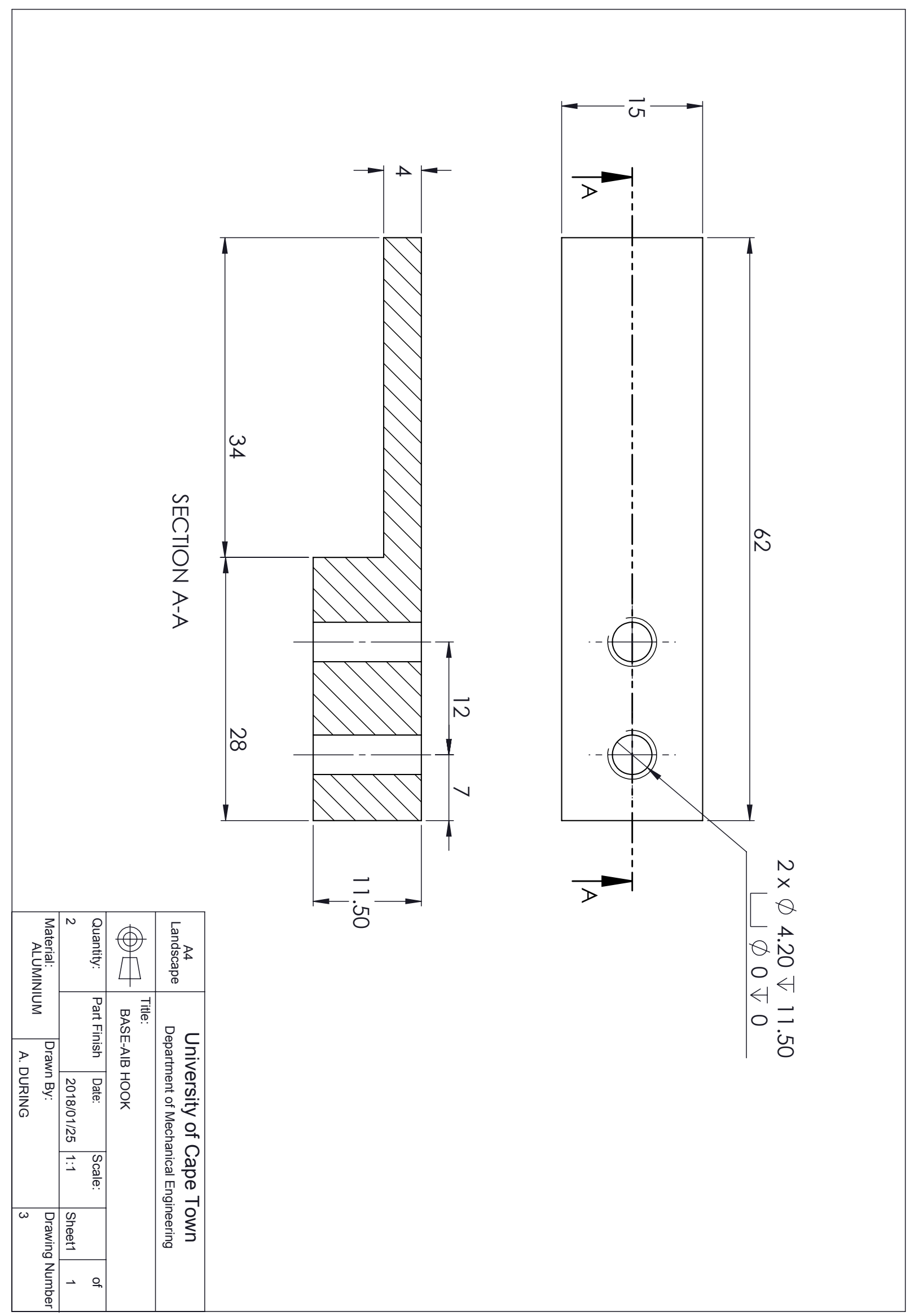




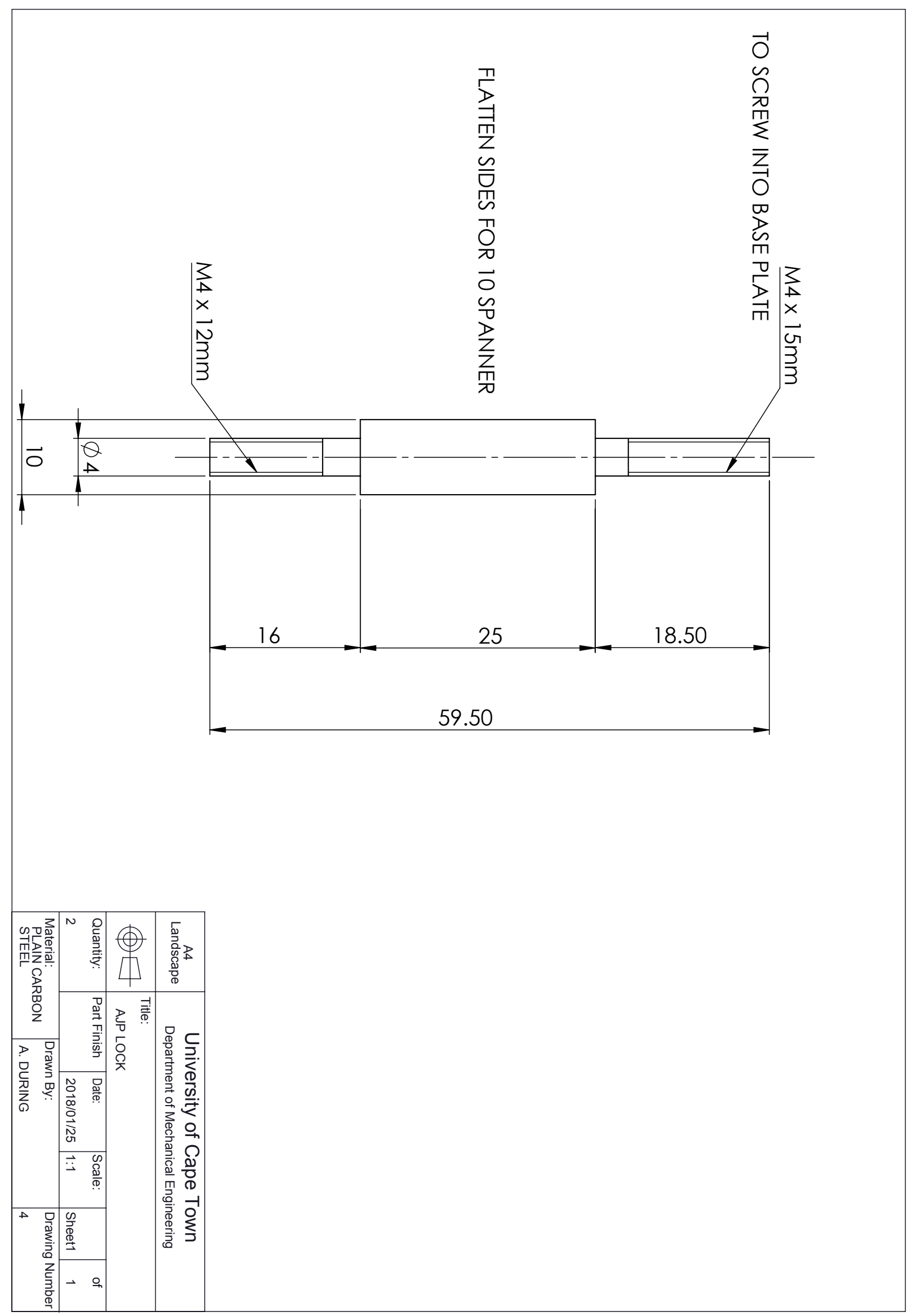




\section{Appendix F}

\section{Ethics Approval Letter}




\section{UNIVERSITY OF CAPE TOWN}

Faculty of Health Sciences

Human Research Ethics Committee

Room E53-46 Old Maln Bullding Groote Schuur Hospltal Observatory 7925 Telephone [021] 4066626 Emall: shuretta,thomasiouct.aciza Website: www health,uct,ac,za/fhs/research/humanethics/forms

12 October 2017

HREC REF! 542/2017

A/Prof G Vlcatos

Mechanical Englneerlng

Electrical \& Mechanical Engineering Bullding

Upper Campus

Dear A/Prof Vicatos

PROJECT TITLE: AN ANALYSIS OF THE MECHANICS OF A NOVEL ANKLE-JOINT PROSTHETIC DURING A STANCE PHASE REPLICATION OF THE TRANS-TIBIAL AMPUTEE LEVEL WALKING GAIT CYCLE (MSC candidate-A During)

Thank you for submltting your response to the Faculty of Health Sclences Human Research Ethics Commlttee recelved on 3 October 2017.

It Is a pleasure to inform you that the HREC has formally approved the above-mentioned study.

Approval is granted for one year untll the $\mathbf{3 0}$ October 2018.

Please submit a progress form, using the standardised Annual Report Form If the study continues beyond the approval perlod. Please submit a Standard Closure form If the study is completed within the approval period.

(Forms can be found on our webslte: www.health.uct,ac,za/fhs/research/humanethlcs/forms)

Please quote the HREC REF in all your correspondence.

Please note that the ongoing ethlcal conduct of the study remalns the responsibillty of the principal Investigator.

Please note that for all studies approved by the HREC, the principal investigator must obtain appropriate instltutional approval, where necessary, before the research may occur.

The HREC acknowledge that the student, Alastalr During will also be Involved In thls study.

Yours slncerely

\section{PROFESSOR M BLOCKMAN}

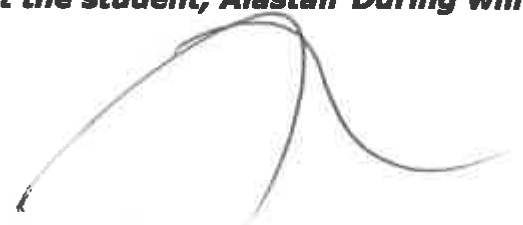

CHAIRPERSON. FHS HUMAN RESEARCH ETHTCS COMMITIEE

Federal Wide Assurance Number: FWA00001637.

Institutional Revlew Board (IRB) number: IRB00001938 
Thls serves to confirm that the University of Cape Town Human Research Ethics Committee complies to the Ethics Standards for Clinical Research wlth a new drug in patients, based on the Medlcal Research Council (MRC-SA), Food and Drug AdmInistration (FDA-USA), International Convention on Harmonisation Good Clinical Practice (ICH GCP), South African Good Clinical Practice Guldellnes (DoH 2006), based on the Assoclation of the British Pharmaceutical Industry Guldellnes (ABPI), and Declaration of Helsinki (2013) guldelines.

The Human Research Ethics Committee granting this approval is in compliance with the ICH Harmonised Trlpartite Guldelines E6: Note for Guldance on Good Clinical Practice (CPMP/ICH/135/95) and FDA Code Federal Regulation Part 50, 56 and 312. 


\section{Appendix G}

\section{Participant Consent Form}




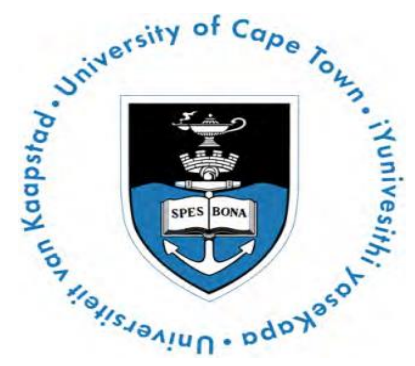

\section{PARTICIPANT INFORMATION SHEET AND INFORMED CONSENT FORM}

\section{FACULTY OF HEALTH SCIENCES: HREC REF no: 542/2017}

An Analysis of the Mechanics of A Novel Ankle-Joint Prosthetic during a Stance Phase Replication of the Trans-Tibial Amputee Level Walking Gait Cycle

\section{Why is this study being done?}

Researchers at the UCT Division of Biomedical Engineering are developing an ankle joint prosthetic. The aim is to improve the ankle function of people with lower-leg amputations. In the long term, it is hoped that this device will improve their ability to walk. The motivation for this is that current ankle prosthetics that are available to low-income amputees in South Africa are not matching the natural ability of the human ankle. Instead, these amputees are forced to rely on uncomfortable walking patterns. These walking styles also cause a $15-25 \%$ increase in energy use when walking.

This device will be combined to two other devices: a prosthetic foot and a 'moonboot'. A 'moonboot' is a device used to prevent movement of a broken ankle. Together these three parts will form a 'fake' prosthetic. This will allow for the simulation of an amputated limb. The 'fake' prosthetic will be secured around your calf and foot, and will stop your ankle from moving within it. By walking with this on it will allow us to test the function of the prosthetic before we involve amputees. It is important for you to know that this is a common way of testing prosthetics.

You have been asked to volunteer for this study because you fall into a category that matches the target population. See "Who can participate in this study?"

\section{Who can participate in this study?}

You can volunteer for this study if you are a healthy male of age $28.4 \pm 5.00$ years, $72.70 \pm 10.34 \mathrm{~kg}$ in weight, and $1.78 \pm 0.06 \mathrm{~m}$ in height.

\section{What is the experiment procedure and length?}

The procedure for this study analyses the prosthetic during a specific walking pattern. This walking pattern is similar to that of an amputee. To analyse the device, motion capture and force feedback equipment will be used.

The comfort of your leg in this device will be ensured when we fit it onto you. You will then be asked to stand, and will be allowed to use waist high guide rails to maintain balance. You will then have a chance to get used to walking with the 'fake' prosthetic. When you are ready you will be asked to do two tests:

1. You will be asked to take three strides from a standing position. These steps will be on straight and flat walkway. The measuring devices will analyse of the ankle device using cameras while you walk. This test will be repeated until 20 suitable strides have been recorded. 
2. You will then be asked to carry out the control test. For this test the 'fake' limb will be removed and the motion markers will be placed on your own limb. You will be asked to repeat the steps explained in Test 1. In this test your natural ankle stiffness will examined.

If you volunteer for the study, you will only need to attend one session. Each session will be divided into three stages. The first is setup and familiarization of 30 minutes. The second is the actual testing of 40 minutes. The last is a post-test debrief, if necessary, of 10 minutes. Thus the total time will be no longer than 80 minutes.

\section{Where will the study take place?}

Testing will be carried out at the UCT's Gait Lab, located at the Sports Science Institute of South Africa.

\section{Who will conduct the experiments?}

This study is being conducted by Mr Alastair During and Associate-Professor George Vicatos, both of UCT.

\section{What are the benefits to you/the world?}

The study will have no direct benefit to you. The testing of the device might benefit the amputees of South Africa.

\section{What are the risks to you?}

The device has been designed with structural safety in mind. You will not experience any pain or discomfort. The only risk is that you might feel unstable when walking with the device. If you fall over, there is risk of bodily harm. To minimize the chances of this happening, safety measures are in place. First, waist high guide rails will be provided. These will provide wobble support should you require it. Second, mattresses will be placed next to the walkway. Finally, one of the researchers will walk with you to provide any support you may need. Thus the risk of injury is low.

As a participant of this study you are covered by the UCT no fault insurance policy. The policy states that UCT will provide you with immediate medical care should you experience any bodily harm caused by participation in the study. It is important for you to know that you have the right to withdraw from this study at any time, for any reason, even if you have already signed the consent form. If you have any complaints or questions about your rights and welfare as a participant in this study you can contact the Human Research Ethics Committee. Those contact details are provided in this consent form.

\section{What happens if you get hurt taking part in this study?}

This research study is covered by an insurance policy taken out by the University of Cape Town. This insurance policy covers you if you incur a bodily injury while taking part in the study. The insurer will pay for all reasonable medical costs required to treat your injury, according to the SA Good Clinical Practice Guidelines 2006, which are based on the Association of the British Pharmaceutical Industry Guidelines. The insurer will pay without you having to prove that the research was responsible for your bodily injury. You may ask the study investigator for a copy of these guidelines.

The insurer will not pay for harm if, during the study, you:

- Do not follow the study investigator's instructions

- Do not take reasonable care of yourself. 
If you are harmed and the insurer pays for the necessary medical costs, usually you will be asked to accept that insurance payment as full settlement of the claim for medical costs. However, accepting this offer of insurance cover does not mean you give up your right to make a separate claim for other losses based on negligence, in a South African court. It is important to follow the study investigator's instructions.

\section{What research related injuries can occur?}

There are no expected physical injuries to you. You might experience some instability, but if so you can make use of the hand rails. If you do fall there is risk of injury to your body, such as a sprained wrist.

\section{What are the costs of participating in the study?}

There will be no cost to you for taking part in this research study.

\section{Do you have to participate in the experiments?}

Taking part in this study is voluntary. You are free to withdraw from this study at ANY time, for ANY reason.

\section{Will travel expenses be provided?}

You will be reimbursed for travel expenses from UCT Medical Campus to the Sports Science Institute of South Africa.

\section{Will participating in the testing grant you post-trial access?}

The results recorded during testing will be purely for the analysis of a prosthetic device. At the end of your testing procedure you will not have access to the device or the data from the study.

\section{If you have any questions}

If you have any questions now or in the future, you may contact Mr Alastair During at +27 7609736269 


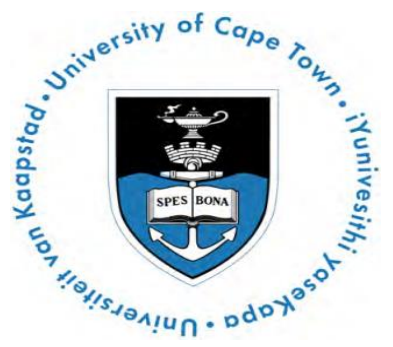

\section{PARTICIPANT INFORMATION SHEET AND INFORMED CONSENT FORM}

\section{FACULTY OF HEALTH SCIENCES: HREC REF no: $(542 / 2017)$}

An Analysis of the Mechanics of a Novel Ankle-Joint Prosthetic during a PseudoStance Phase Replication of the Trans-Tibial Amputee Level Walking Gait Cycle

\section{Statement of Understanding}

I confirm that the exact procedure and the possible complications of the above tests have been thoroughly explained to me. I am free to withdraw from the study at any time should I choose to do so. I understand that I may ask questions at any time during the testing procedure. I know that the personal information required by the researchers and derived from the testing procedure will remain strictly confidential and will only be revealed as a number in statistical analysis. I have carefully read this form and understand the nature, purpose and procedures of this study. I agree to participate in this testing procedure conducted by the UCT Division of Biomedical Engineering at the Sports Science Institute of South Africa.

\section{Consent to participate in a Research Study:}

To voluntarily agree to take part in this study, you must sign in the space provided below. If you choose to participate, you may withdraw at any time. You are not giving up any of your legal rights by signing this form. Your signature below indicates that you have read, or had read to you, this entire consent form, including the risks and benefits, and have had all of your questions, if any, answered.

\begin{tabular}{|l|l|l|}
\hline Printed Name of Volunteer: & & Date: \\
\hline Signature of Volunteer: & & Time: \\
\hline Printed Name of Witness & & Date: \\
\hline Signature of Witness & & Time: \\
\hline Printed Name of Investigator: & & Date: \\
\hline Signature of Investigator: & & Time: \\
\hline
\end{tabular}

I guarantee that this translation is accurate.

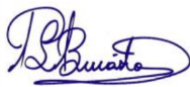

Associate-Professor George Vicatos

Department of Mechanical Engineering

2nd level Electrical-Mechanical Building

Library Road

Upper Campus University of Cape Town

Rondebosch, 7701, South Africa

Tel: +27(0)216503231

Fax: +27(0)21 6503240 


\section{Contact details of all researchers:}

All the researchers are from the University of Cape Town. The postal address of the University of Cape Town and individual contact details of all the researcher are:

\section{Faculty of Health Sciences \\ University of Cape Town \\ Observatory, 7925 \\ South Africa}

\begin{tabular}{|c|c|c|c|}
\hline $\begin{array}{l}\text { Title, first name, } \\
\text { surname }\end{array}$ & $\begin{array}{l}\text { Department/Division } \\
\text { at UCT }\end{array}$ & E-mail & Address \\
\hline $\begin{array}{l}\text { A/Professor George } \\
\text { Vicatos }\end{array}$ & $\begin{array}{l}\text { Mechanical } \\
\text { Engineering }\end{array}$ & George.vicatos@uct.ac.za & $\begin{array}{l}\text { Department of Mechanical } \\
\text { Engineering } \\
\text { 2nd level Electrical-Mechanical } \\
\text { Building }\end{array}$ \\
\hline Mr Alastair During & $\begin{array}{l}\text { Biomedical } \\
\text { Engineering }\end{array}$ & Drnala001@myuct.ac.za & $\begin{array}{l}\text { Library Road } \\
\text { Upper Campus University of Cape } \\
\text { Town } \\
\text { Rondebosch, 7701, South Africa }\end{array}$ \\
\hline
\end{tabular}

Contact details of University of Cape Town Faculty of Health Sciences Human Research and Ethics Committee: Please contact the University of Cape Town Faculty of Health Sciences Human Research and Ethics Committee (HREC) if you have any questions or concerns about your rights or welfare as a research participant.

Human Research Ethics Committee

E 52, Room 24, Old Main Building, Groote Schuur Hospital, Observatory

Telephone: 27214066338

Fax: 27214066411

Email: shuretta.thomas@uct.ac.za 


\section{Appendix $\mathrm{H}$}

\section{Participant Questionnaire}




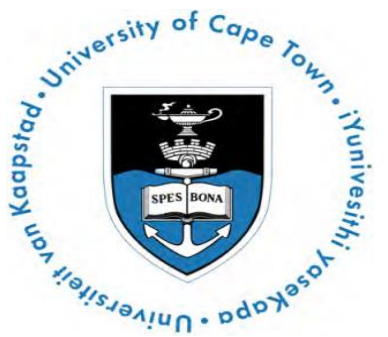

PARTICIPANT QUESTIONNAIRE FORM

FACULTY OF HEALTH SCIENCES: 542/2017

An Analysis of the Mechanics of A Novel Ankle-Joint Prosthetic during a Stance Phase Replication of the Trans-Tibial Amputee Level Walking Gait Cycle.

\section{Purpose of Questionnaire}

The purpose of the questionnaire is to record the demographic data of each participant used in the trial.

What will be done with my answers?

The answers of the participant will be used to aid the analysis of the results his ankle rollover characteristics, both with and without the pseudo-prosthesis.

Will there be confidentiality?

The name, contact, email and location details will be asked purely for contact purposes between the researcher and participant. The name, contact, email and location details will not be included in any document or research and will be discarded once communication between researcher and participant has ceased.

All subsequent questions (relevant to the study) will be purely based on the demographic and health characteristics of the participant and the answers will be kept in a secure password protected computer.

I, Alastair During, promise to use the name, location, email and contact details of any participant who fills out this questionnaire for the sole purpose of contacting the participant and will be discarded once communication between researcher and participant has ceased.

\section{Do I need to answer every question?}

You are NOT required to answer every question, however your results obtained during the research study will be included in the study depending on the answers you submit on the questionnaire. 


\section{QUESTIONNAIRE}

\begin{tabular}{|c|c|c|}
\hline Question & Answer & Example \\
\hline Name & & \\
\hline $\begin{array}{c}\text { Telephone Number or } \\
\text { Cell Number }\end{array}$ & & \\
\hline Email & & \\
\hline Gender & Age & - Male 'M' or Female 'F' \\
\hline Weight & & \\
\hline Height & - Kg's \\
\hline $\begin{array}{c}\text { Do you consider your } \\
\text { walking gait to be } \\
\text { 'abnormal'? If so, please } \\
\text { explain. }\end{array}$ & & cm \\
\hline $\begin{array}{c}\text { Have you ever had a } \\
\text { significant injury to your } \\
\text { hips or legs? If so, please } \\
\text { explain. }\end{array}$ & $\begin{array}{c}\text { Do you ever experience } \\
\text { difficulties with walking? } \\
\text { If so, please explain. }\end{array}$ & i.e. "I have a slight limp" \\
\hline
\end{tabular}


Appendix I

Statistical Analysis Methodology

(Example) 
Initial Outputs from Data Processing (Participant 2)

\begin{tabular}{|c|c|c|c|c|c|c|c|c|c|c|c|c|c|c|c|c|c|c|c|c|}
\hline & $\begin{array}{l}\text { B_Stride_ } \\
\text { Length }\end{array}$ & ${ }_{y}^{\text {E_Velocit }}$ & $\stackrel{D_{D F}{ }^{E} P_{-} C}{ }$ & ${ }_{\mathrm{PF}}^{\mathrm{E} \mathrm{SP}_{-} \mathrm{C}}$ & 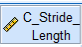 & ${ }_{y}^{C}$ CVelocit & it $\Rightarrow \begin{array}{c}\text { C_SP_C } \\
\text { DF }\end{array}$ & $\begin{array}{c}\text { C_SP_C } \\
\mathrm{PF}^{2}\end{array}$ & ${ }_{-S}^{\text {Exp_CDF }}$ & $=\operatorname{Exp}_{-S}$ & ${ }_{-T}^{\text {Exp_CDF }}$ & $=\underbrace{\text { Exp_CPF }}_{-T}$ & $=\underset{A}{\operatorname{Exp} p_{-} \mathrm{CDF}}$ & $=\underset{A}{\text { Exp_CPF }}$ & $={ }^{\mathrm{Con} \_\mathrm{CDF}}$ & $={ }_{-S}^{\text {Con_CPF }}$ & $\$_{-T}^{\text {Con_CDF }}$ & ${ }_{-T}^{\text {Con_CPF }}$ & $\underbrace{\text { Con_CDF }}_{-A}$ & $\mathrm{~F} \otimes_{-A}^{\mathrm{Con} C \mathrm{CPF}}$ \\
\hline 1 & 1.27 & .81 & 85.90 & 8.55 & 1.23 & .88 & 84.35 & $\begin{array}{l}6.52 \\
\end{array}$ & $\quad 3.81$ & -10 & 1.04 & -02 & 15.71 & -10.42 & 8.07 & -.73 & 1.06 & -10 & 7.50 & -8.18 \\
\hline 2 & 1.26 & .72 & 85.47 & 10.03 & 1.27 & .84 & 84.27 & 6.85 & 3.53 & -13 & 1.06 & -.02 & 17.25 & -8.18 & 7.58 & -.44 & 1.01 & -.06 & 7.61 & -8.05 \\
\hline 3 & 1.29 & .96 & 84.65 & $8.8 \leftarrow$ & & & & & 3.58 & -.11 & 1.03 & -.02 & 16.52 & -8.96[ & & & & & & \\
\hline 4 & 1.24 & .83 & 83.11 & 8.68 & 1.28 & .89 & 82.79 & 7.91 & 3.68 & -.04 & 1.04 & -.01 & 16.21 & -8.79 & 6.44 & -.68 & 89 & -.07 & 7.90 & -6.30 \\
\hline 5 & 1.24 & .75 & 84.25 & 7.48 & 1.24 & .63 & 81.48 & 5.09 & 3.72 & -.22 & 1.02 & -.04 & 15.76 & -11.19 & 9.05 & .67 & 1.14 & .08 & 7.20 & -6.91 \\
\hline 6 & 1.21 & .82 & 84.55 & 9.55 & 1.21 & .84 & 80.09 & 7.24 & 3.74 & -10 & 1.04 & -.01 & 15.96 & -8.27 & 7.83 & -66 & 1.05 & -.07 & 7.66 & -6.49 \\
\hline 7 & 1.27 & .85 & 85.26 & 8.37 & 1.19 & .80 & 82.82 & 9.25 & 3.83 & -14 & 1.07 & -.03 & 16.00 & -10.89 & 7.43 & -.52 & .99 & -.06 & 7.61 & -6.42 \\
\hline 8 & 1.31 & .84 & 83.92 & 8.24 & 1.17 & .80 & 82.61 & 10.00 & 4.05 & -19 & 1.10 & -.04 & 15.62 & -12.65 & 7.12 & -.31 & 1.01 & -.04 & 8.16 & -6.80 \\
\hline 9 & 1.24 & .84 & 82.86 & 8.16 & 1.26 & .83 & 84.71 & 9.02 & 4.00 & -.05 & 1.10 & -.01 & 15.71 & -10.28 & 7.39 & -.51 & 1.03 & -.07 & 7.99 & -7.26 \\
\hline 10 & 1.10 & .83 & 85.48 & 8.30 & 1.20 & .84 & 84.48 & 9.91 & 3.98 & -26 & 1.08 & -.05 & 15.50 & -12.04 & 7.67 & -39 & 1.01 & -.05 & 7.55 & -7.74 \\
\hline 11 & 1.35 & .84 & 85.66 & 7.79 & 1.31 & .71 & 83.12 & 10.39 & 3.70 & -25 & 1.06 & -.06 & 16.42 & -13.99 & 7.40 & -.44 & 1.08 & -.06 & 8.35 & -7.29 \\
\hline 12 & 1.22 & .83 & 85.53 & 8.09 & 1.28 & .81 & 86.06 & 5.58 & 3.47 & -16 & .99 & -.03 & 16.31 & -11.95 & 8.88 & $\begin{array}{l}-.44 \\
-42\end{array}$ & 1.02 & -.06 & 6.55 & -8.30 \\
\hline 13 & 1.15 & .74 & 82.67 & 8.89 & 1.35 & .67 & 83.46 & 6.54 & 3.56 & -28 & .95 & -05 & 15.28 & -10.57 & 6.02 & $\begin{array}{r}-.59 \\
-59\end{array}$ & 1.03 & -.05 & 9.79 & -4.62 \\
\hline 14 & 1.21 & .81 & 86.15 & 8.23 & 1.26 & .72 & 82.11 & 8.95 & 3.70 & -16 & 1.03 & -.03 & 15.95 & -8.74 & 5.96 & -29 & 90 & -.02 & 8.67 & -4.25 \\
\hline 15 & 1.25 & .90 & 83.89 & 9.00 & 1.17 & .68 & 85.05 & 5.98 & 3.84 & -.03 & 1.05 & .00 & 15.69 & -7.26 & 8.19 & -.63 & 99 & -.09 & 6.92 & -8.24 \\
\hline
\end{tabular}

Example of Identified Outlier

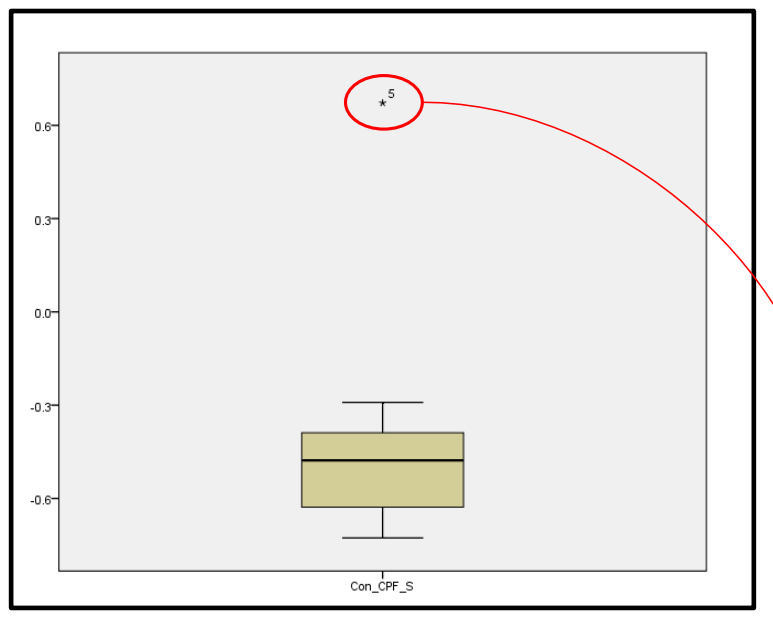

Initial analysis of raw data revealed that Control trial \#3 had errors with recording and was thus removed completely.

CPF stiffness of Control trial \#5 was identified as an outlier, as it is greater than the inter-quartile range by a factor $>x 3$.

Therefor Control trial \#5 is completely removed from results, leaving the dataset of Participant 2 to consist of 13 samples.

Results After Removal of Outliers

\begin{tabular}{|c|c|c|c|c|c|c|c|c|c|c|c|c|c|c|c|c|c|c|c|c|c|}
\hline & $\begin{array}{c}\text { B_Stride } \\
\text { Length }\end{array}$ & $-{ }_{y}^{\text {E_Velocit }}$ & 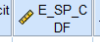 & ${ }_{\mathrm{PF}}^{\mathrm{ESP}}{ }^{\mathrm{S}}$ & \& ${ }_{\text {Length }}^{\text {CStride }}$ & -2 C_Velocit & it 2 C_SP_C & ${ }_{\text {PF_C }}^{\text {C_SPC }}$ & $\begin{array}{c}\text { Exp_CDF } \\
-S\end{array}$ & $\underbrace{\text { Exp_CPF }}_{S}$ & $=\underset{-T}{\text { Exp_CDF }}$ & $=\underbrace{\text { Exp_CPF }}_{-T}$ & $=\underset{-A}{\operatorname{Exp} C D F}$ & $=\underset{A}{\operatorname{Exp}{ }_{-} \mathrm{CPF}}$ & $\otimes_{-\mathrm{S}}^{\text {Con_CDF }}$ & & on_CPF & $\$_{-T}^{\text {Con_CDF }}$ & $\underbrace{\text { Con_CPF }}_{-}$ & $=\underbrace{\text { Con_CDF }}_{-A}$ & F ${ }_{-A}^{\text {Con_CPF }}$ \\
\hline 1 & 1.27 & 81 & $31 \quad 85.90$ & 8.55 & $\begin{array}{l}5 \\
5.23\end{array}$ & .88 & $\begin{array}{l}38 \\
\quad 84.35 \\
\end{array}$ & 6.52 & 3.81 & \begin{tabular}{|r|}
-10 \\
\end{tabular} & 1.04 & $\begin{array}{ll}4 & -.02\end{array}$ & $2 \quad 15.71$ & $1 \quad-10.42$ & 8.07 & & -.73 & 1.06 & -10 & 7.50 & $\begin{array}{ll}0 & -8.18\end{array}$ \\
\hline 2 & & & & & 1.27 & .84 & 84.27 & 6.85 & & & & & & & 7.58 & & -44 & 1.01 & -.06 & 7.61 & -8.05 \\
\hline 3 & & & & & & & & & & & & & & & & & . & & & & \\
\hline 4 & 1.24 & .83 & 83.11 & 8.68 & 1.28 & .89 & 82.79 & 7.91 & 3.68 & -.04 & 1.04 & -.01 & 16.21 & -8.79 & 6.44 & 1 & -68 & .89 & -.07 & 7.90 & -6.30 \\
\hline 5 & 1.24 & .75 & 84.25 & 7.4 & & 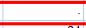 & & 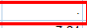 & 3.72 & -.22 & 1.02 & -.04 & 15.76 & -11.19[ & & & & & & & \\
\hline 6 & 1.21 & .82 & 84.55 & 9.55 & 1.21 & .84 & 80.09 & 7.24 & 3.74 & -10 & 1.04 & -.01 & 15.96 & -8.27 & 7.83 & & -.66 & 1.05 & -.07 & 7.66 & -6.49 \\
\hline 7 & 1.27 & .85 & 85.26 & 8.37 & 1.19 & .80 & 82.82 & 9.25 & 3.83 & -14 & 1.07 & -.03 & 16.00 & -10.89 & 7.43 & & -.52 & .99 & -.06 & 7.61 & -6.42 \\
\hline 8 & 1.31 & .84 & 83.92 & 8.24 & 1.17 & .80 & 82.61 & 10.00 & 4.05 & -19 & 1.10 & -.04 & 15.62 & -12.65 & 7.12 & & -31 & 1.01 & -.04 & 8.16 & -6.80 \\
\hline 9 & 1.24 & .84 & 82.86 & 8.16 & 1.26 & .83 & 84.71 & 9.02 & 4.00 & -.05 & 1.10 & -01 & 15.71 & -10.28 & 7.39 & & -.51 & 1.03 & -.07 & 7.99 & -7.26 \\
\hline 10 & 1.10 & .83 & 85.48 & 8.30 & 1.20 & .84 & 84.48 & 9.91 & 3.98 & -26 & 1.08 & -.05 & 15.50 & -12.04 & 7.67 & & -39 & 1.01 & -05 & 7.55 & -7.74 \\
\hline 11 & 1.35 & .84 & 85.66 & 7.79 & 1.31 & .71 & 83.12 & 10.39 & 3.70 & -.25 & 1.06 & -06 & 16.42 & -13.99 & 7.40 & & -44 & 1.08 & -.06 & 8.35 & -7.29 \\
\hline 12 & 1.22 & .83 & 85.53 & 8.09 & 1.28 & .81 & 86.06 & 5.58 & 3.47 & -16 & .99 & -03 & 16.31 & -11.95 & 8.88 & & -42 & 1.02 & -.06 & 6.55 & -8.30 \\
\hline 13 & 1.15 & .74 & 82.67 & 8.89 & 1.35 & .67 & 83.46 & 6.54 & 3.56 & -28 & .95 & -05 & 15.28 & -10.57 & 6.02 & & -59 & 1.03 & -05 & 9.79 & -4.62 \\
\hline 14 & 1.21 & .81 & 86.15 & 8.23 & 1.26 & .72 & 82.11 & 8.95 & 3.70 & -16 & 1.03 & -03 & 15.95 & -8.74 & 5.96 & & -29 & .90 & -02 & 8.67 & -4.25 \\
\hline 15 & 1.25 & .90 & 83.89 & 9.00 & 1.17 & .68 & 85.05 & 5.98 & 3.84 & -.03 & 1.05 & .00 & 15.69 & -7.26 & 8.19 & & -.63 & .99 & -.09 & 6.92 & -8.24 \\
\hline
\end{tabular}

Shapiro-Wilk Normality Test Results

\begin{tabular}{|c|c|c|c|c|c|c|}
\hline \multicolumn{7}{|c|}{ Tests of Normality } \\
\hline & \multicolumn{3}{|c|}{ Kolmogorov-Smirnov ${ }^{a}$} & \multicolumn{3}{|c|}{ Shapiro-Wilk } \\
\hline & Statistic & df & Sig. & Statistic & df & Sig. \\
\hline C_Stride_Length & .123 & 13 & $.200^{x}$ & .959 & 13 & .742 \\
\hline C_Velocity & .233 & 13 & .051 & .891 & 13 & .102 \\
\hline C_SP_CDF & .148 & 13 & $.200^{*}$ & .961 & 13 & .773 \\
\hline C_SP_CPF & .175 & 13 & $.200^{*}$ & .924 & 13 & .288 \\
\hline Con_CDF_S & .195 & 13 & .190 & .946 & 13 & .539 \\
\hline Con_CPF_S & .144 & 13 & $.200^{*}$ & .956 & 13 & .692 \\
\hline Con_CDF_T & .227 & 13 & .065 & .872 & 13 & .055 \\
\hline Con_CPF_T & .133 & 13 & $.200^{*}$ & .978 & 13 & .970 \\
\hline Con_CDF_A & .168 & 13 & $.200^{*}$ & .934 & 13 & .389 \\
\hline Con_CPF_A & .164 & 13 & $.200^{*}$ & .881 & 13 & .074 \\
\hline \multicolumn{7}{|c|}{ *. This is a lower bound of the true significance. } \\
\hline
\end{tabular}

The S-W tests reveal that all variables have normally distributed datasets $(p>0.05)$ 
Variable Means + Standard Deviation

\begin{tabular}{|c|c|c|c|c|c|}
\hline \multicolumn{5}{|c|}{ Group Statistics } & \\
\hline & & $N$ & Mean & Std. Deviation & Mean \\
\hline \multirow[t]{2}{*}{ Stride_Length } & $E$ & 13 & 1.2365 & .06264 & .01737 \\
\hline & $\mathrm{C}$ & 13 & 1.2449 & .05516 & .01530 \\
\hline \multirow[t]{2}{*}{ Velocity } & $E$ & 13 & .8220 & .04050 & .01123 \\
\hline & $\mathrm{C}$ & 13 & .7928 & .07403 & .02053 \\
\hline \multirow[t]{2}{*}{ SP_CDF } & $E$ & 13 & 84.5540 & 1.20210 & .33340 \\
\hline & $\mathrm{C}$ & 13 & 83.5315 & 1.53129 & .42470 \\
\hline \multirow[t]{2}{*}{ SP_CPF } & $E$ & 13 & 8.4079 & .53694 & .14892 \\
\hline & $\mathrm{C}$ & 13 & 8.0109 & 1.65933 & .46022 \\
\hline \multirow[t]{2}{*}{ CDF_S } & $E$ & 13 & 3.7745 & .16795 & .04658 \\
\hline & $\mathrm{C}$ & 13 & 7.3839 & .84357 & .23396 \\
\hline \multirow[t]{2}{*}{ CPF_S } & $E$ & 13 & -.1527 & .08355 & .02317 \\
\hline & $\mathrm{C}$ & 13 & -.5085 & .14073 & .03903 \\
\hline \multirow[t]{2}{*}{ CDF_T } & $E$ & 13 & 1.0441 & .04196 & .01164 \\
\hline & $\mathrm{C}$ & 13 & 1.0044 & .05480 & .01520 \\
\hline \multirow[t]{2}{*}{ CPF_T } & $E$ & 13 & -.0299 & .01906 & .00529 \\
\hline & $\mathrm{C}$ & 13 & -.0619 & .02139 & .00593 \\
\hline \multirow[t]{2}{*}{ CDF_A } & $E$ & 13 & 15.8553 & .32746 & .09082 \\
\hline & $\mathrm{C}$ & 13 & 7.8675 & .80287 & .22268 \\
\hline \multirow[t]{2}{*}{ CPF_A } & $E$ & 13 & -10.5400 & 1.90235 & .52762 \\
\hline & $\mathrm{C}$ & 13 & -6.9183 & 1.31262 & .36406 \\
\hline
\end{tabular}

$\underline{\text { Final Independent T-Test Results }}$

\begin{tabular}{|c|c|c|c|c|c|c|c|c|c|c|}
\hline & & \multicolumn{7}{|c|}{ Independent Samples Test } & & \\
\hline & & \multicolumn{5}{|c|}{$\begin{array}{l}\text { Levene's Test for Equality of } \\
\text { Variances }\end{array}$} & \multicolumn{4}{|c|}{ t-test for Equality of Means } \\
\hline & & \multirow[b]{2}{*}{$\mathrm{F}$} & \multirow[b]{2}{*}{ Sig. } & \multirow[b]{2}{*}{$t$} & \multirow[b]{2}{*}{ df } & \multirow[b]{2}{*}{ Sig. (2-tailed) } & \multirow{2}{*}{$\begin{array}{l}\text { Mean } \\
\text { Difference }\end{array}$} & \multirow{2}{*}{$\begin{array}{l}\text { Std. Error } \\
\text { Difference }\end{array}$} & \multicolumn{2}{|c|}{$\begin{array}{l}95 \% \text { Confidence Interval of the } \\
\text { Difference }\end{array}$} \\
\hline & & & & & & & & & Lower & Upper \\
\hline \multirow[t]{2}{*}{ Stride_Length } & $\begin{array}{l}\text { Equal variances } \\
\text { assumed }\end{array}$ & .005 & .945 & -362 & 24 & .721 & -.00838 & .02315 & -.05615 & .03940 \\
\hline & $\begin{array}{l}\text { Equal variances not } \\
\text { assumed }\end{array}$ & & & -.362 & 23.622 & .721 & -.00838 & .02315 & -.05620 & .03944 \\
\hline \multirow[t]{2}{*}{ Velocity } & $\begin{array}{l}\text { Equal variances } \\
\text { assumed }\end{array}$ & 6.499 & .018 & 1.247 & 24 & .225 & .02918 & .02340 & -.01912 & .07748 \\
\hline & $\begin{array}{l}\text { Equal variances not } \\
\text { assumed }\end{array}$ & & & 1.247 & 18.592 & .228 & .02918 & .02340 & -.01988 & .07824 \\
\hline \multirow[t]{2}{*}{ SP_CDF } & $\begin{array}{l}\text { Equal variances } \\
\text { assumed }\end{array}$ & .320 & .577 & 1.894 & 24 & .070 & 1.02256 & .53994 & -.09181 & 2.13693 \\
\hline & $\begin{array}{l}\text { Equal variances not } \\
\text { assumed }\end{array}$ & & & 1.894 & 22.719 & .071 & 1.02256 & .53994 & -.09515 & 2.14026 \\
\hline \multirow[t]{2}{*}{ SP_CPF } & $\begin{array}{l}\text { Equal variances } \\
\text { assumed }\end{array}$ & 25.103 & .000 & .821 & 24 & .420 & 39708 & 48371 & -.60125 & 1.39541 \\
\hline & $\begin{array}{l}\text { Equal variances not } \\
\text { assumed }\end{array}$ & & & .821 & 14.486 & .425 & .39708 & .48371 & -.63712 & 1.43129 \\
\hline \multirow[t]{2}{*}{ CDF_S } & $\begin{array}{l}\text { Equal variances } \\
\text { assumed }\end{array}$ & 9.745 & .005 & -15.130 & 24 & .000 & -3.60945 & .23856 & -4.10180 & -3.11709 \\
\hline & $\begin{array}{l}\text { Equal variances not } \\
\text { assumed }\end{array}$ & & & -15.130 & 12.950 & .000 & -3.60945 & .23856 & -4.12502 & -3.09388 \\
\hline \multirow[t]{2}{*}{ CPF_S } & $\begin{array}{l}\text { Equal variances } \\
\text { assumed }\end{array}$ & 4.213 & .051 & 7.839 & 24 & .000 & .35583 & .04539 & .26215 & 44951 \\
\hline & $\begin{array}{l}\text { Equal variances not } \\
\text { assumed }\end{array}$ & & & 7.839 & 19.524 & .000 & .35583 & .04539 & .26100 & .45066 \\
\hline \multirow[t]{2}{*}{ CDF_T } & $\begin{array}{l}\text { Equal variances } \\
\text { assumed }\end{array}$ & .465 & .502 & 2.078 & 24 & .049 & .03977 & .01914 & .00026 & .07928 \\
\hline & $\begin{array}{l}\text { Equal variances not } \\
\text { assumed }\end{array}$ & & & 2.078 & 22.473 & .049 & .03977 & .01914 & .00012 & .07942 \\
\hline \multirow[t]{2}{*}{$\mathrm{CPF}_{-} \mathrm{T}$} & $\begin{array}{l}\text { Equal variances } \\
\text { assumed }\end{array}$ & .033 & .856 & 4.026 & 24 & .000 & .03199 & .00795 & .01559 & .04839 \\
\hline & $\begin{array}{l}\text { Equal variances not } \\
\text { assumed }\end{array}$ & & & 4.026 & 23.688 & .001 & .03199 & .00795 & .01558 & .04840 \\
\hline \multirow[t]{2}{*}{ CDF_A } & $\begin{array}{l}\text { Equal variances } \\
\text { assumed }\end{array}$ & 3.568 & .071 & 33.216 & 24 & .000 & 7.98783 & .24048 & 7.49149 & 8.48416 \\
\hline & $\begin{array}{l}\text { Equal variances not } \\
\text { assumed }\end{array}$ & & & 33.216 & 15.885 & .000 & 7.98783 & .24048 & 7.47773 & 8.49793 \\
\hline \multirow[t]{2}{*}{ CPF_A } & $\begin{array}{l}\text { Equal variances } \\
\text { assumed }\end{array}$ & 1.320 & .262 & -5.650 & 24 & .000 & -3.62168 & .64103 & -4.94470 & -2.29866 \\
\hline & $\begin{array}{l}\text { Equal variances not } \\
\text { assumed }\end{array}$ & & & -5.650 & 21.315 & .000 & -3.62168 & .64103 & -4.95357 & -2.28979 \\
\hline
\end{tabular}


Appendix $\mathbf{J}$

Additional Results Figures 

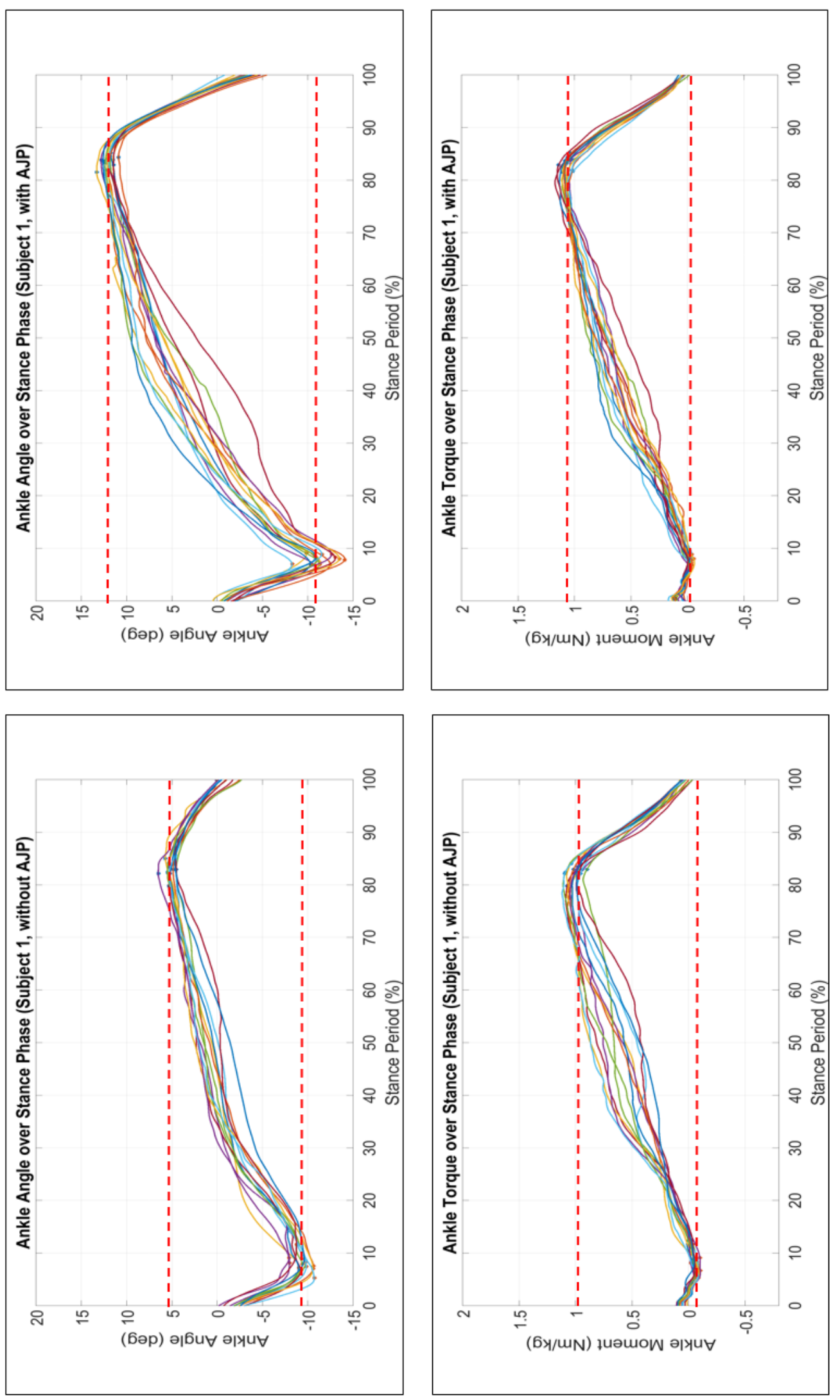

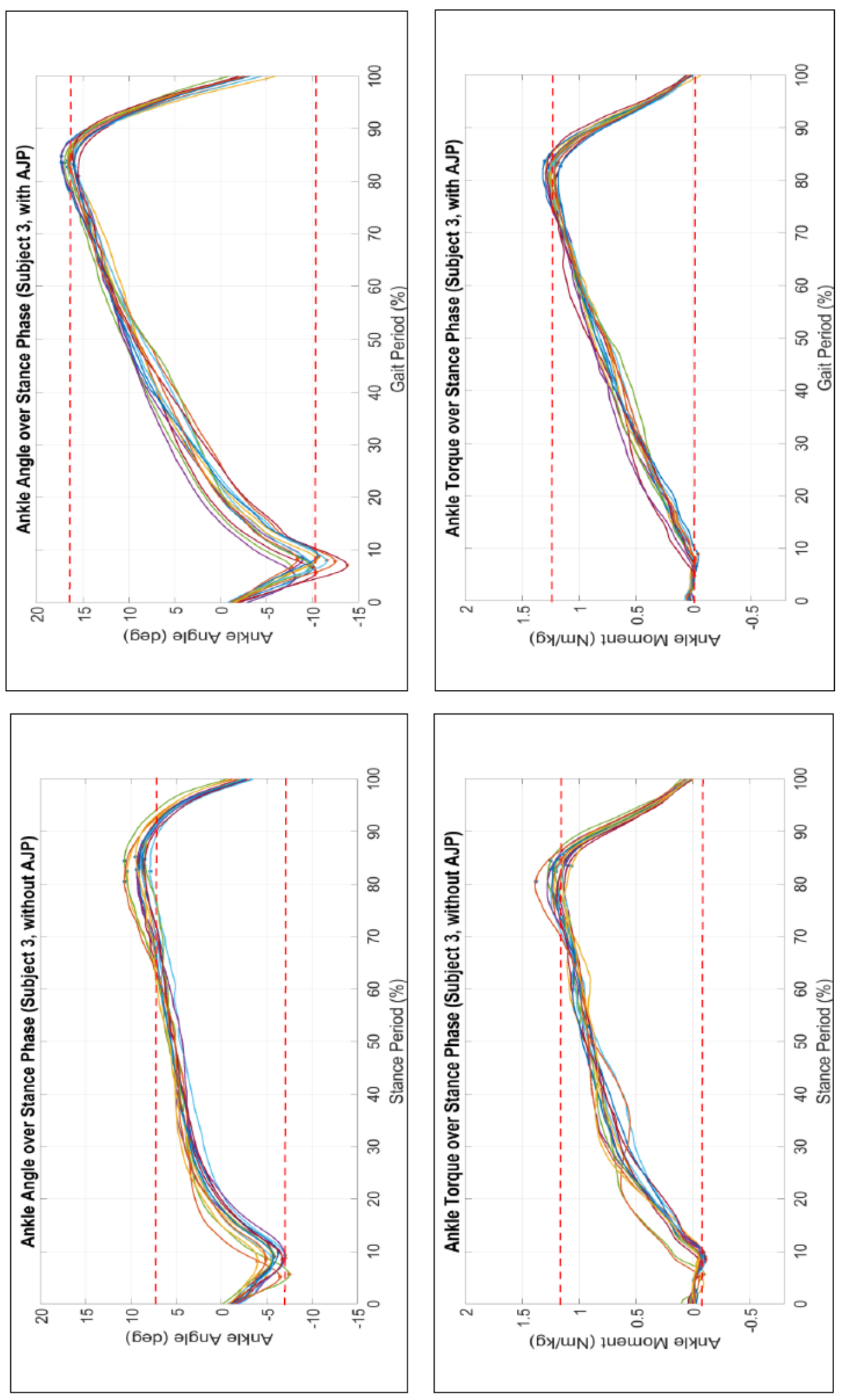